GRAPHICS

STANDARDS IN THE

COMPUTER-AIDED

ACQUISITION AND

LOGISTIC SUPPORT

(CALS) PROGRAM,

FISCAL YEAR 1989

VOLUME 1: TEST

REQUIREMENTS

DOCUMENT,

EXTENDED CGM

(CGEM)
Daniel R. Benigni Editor

U.S. DEPARTMENT OF COMMERCE Natlonal Institute of Standards and Technology

Natlonal Computer Systems Laboratory Galthersburg, MD 20899
U.S. DEPARTMENT OF COMMERCE Robert A. Mosbacher, Secretary NATIONAL INSTITUTE OF STANDARDS AND TECHNOLOGY

John W. Lyons, Director 



\section{GRAPHICS}

\section{STANDARDS IN THE}

COMPUTER-AIDED

ACQUISITION AND

LOGISTIC SUPPORT

\section{(CALS) PROGRAM,} FISCAL YEAR 1989

VOLUME 1: TEST

REQUIREMENTS

DOCUMENT,

\section{EXTENDED CGM}

\section{(CGEM)}

Daniel R. Benigni Editor

U.S. DEPARTMENT OF COMMERCE National Institute of standards and Technology Natlonal Computer Systems Laboratory Galthersburg, MD 20899

May 1990

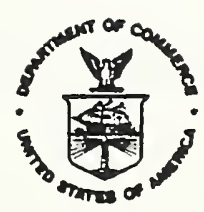

U.S. DEPARTMENT OF COMMERCE Robert A. Mosbacher, Secretary MATIONAL INSTITUTE OF STANDARDS AND TECHNOLOQY

John W. Lyons, Director 



\section{EXECUTIVE SUMMARY}

The overall objective of the Department of Defense Computer-aided Acquisition and Logistic support (CALS) Program is to integrate the design, manufacturing, and logistic functions through the efficient application of computer technology. NIST has been funded since spring 1986 to recommend a suite of industry standards for system integration and digital data transfer, and to accelerate their implementation.

During FY86 NIST tasks for CALS in the area of graphics standards focused on identification of recommended standards to OSD which would be applicable to the DoD environment; comparison of graphics standards among themselves and with product data standards; and the status of ongoing graphics standards efforts as well as related validation efforts'. 'CALS' needs for graphics standards were assessed, and an architecture for their inclusion into specific CALS programs was created. Finally, a plan was recommended for accelerating the development, related validation efforts and implementation of graphics standards into the CALS program.

Building on the knowledge and experience gained during FY86, NIST tasks for CALS in the area of graphics standards in FY87 emphasized the particular graphics standard dealing with the transfer of pictorial data from one system to another, namely the computer Graphics Metafile (CGM) standard (FIPS PUB 128) ${ }^{2}$. Graphics tasks included an assessment of raster-to-vector conversion technology, and where the CGM might fit into that process; efforts toward development of CGM validation routines; injection of CALS requirements into the Extended CGM (CGEM) standards work, as well as into the CGM Registration process. In addition, functional requirements and conceptual design documents were completed for a reference implementation for CGM; a design specification was created for an IGES-to-CGM translator; and a preliminary CALS Application Profile for CGM was formed from the application profile work of the MAP/TOP organization.

During FY88, NIST tasks for CALS in the graphics standards area were in large measure a continuation of those efforts begun the

${ }^{1}$ Kemmerer, S., Editor, "Final NBS Report for CALS, FY86," U.S. Department of Commerce, National Bureau of standards, NBSIR 873566, May 1987 .

'Kemmerer, S., Editor, "A collection of Technical studies Completed for the Computer-aided Acquisition and Logistic support (CALS) Program, Fiscal Year 1987," U.S. Department of Commerce, National Bureau of Standards, NBSIR 88-3727, March 1988 . 
year before ${ }^{3}$. Final text was completed for the initial publication of a Military Specification which is the CALS Application Profile for CGM. NIST continued to participate in graphics standards work in support of CALS requirements in the areas of CGM conformance testing, the CGEM, and CGM Registration. In the particular area of CGM conformance testing, the needs for testing both to FIPS 128 and to the Application Profile for CGM were identified; existing commercial implementations of CGM were analyzed and compared functionally to both CGM and Application Profile requirements; and required CGM conformance testing tasks were described in detail, responsibilities in the testing process delineated, and the impact of CGM testing was assessed both for CALS and the commercial marketplace.

This collection of reports represents the continuing efforts of the Graphics Software Group of NIST/NCSL in FY89 in support of computer graphics standards for CALS, and in particular $\mathrm{CGM}^{4}$. It provides a progress report on continuing graphics standards efforts related to the Computer Graphics Metafile (CGM) standard, including the Extended CGM (CGEM), Graphics Registration, and the CGM Application Profile for CALS (or MIL-D-28003). In addition, the creation of a Test Requirements Document for MIL-D-28003 is detailed. This Test Requirements Document will provide the basis for developing conformance tests to determine compliance with MILD-28003.

This report is subdivided into four separate final cals deliverables, entitled as follows:

1. Test Requirements Document for CALS CGM Conforming Basic Metafiles

2. Injection of CALS Requirements in the Extended CGM (CGEM) Standards Work

3. MIL-D-28003 Revision Recommendations

4. CGM Registration in support of CALS Requirements

${ }^{3}$ Morgan, Roy S., Editor, "A Collection of Technical studies Completed for the Computer-aided Acquisition and Logistic Support (CALS) Program, Fiscal Year 1988, U.S. Department of Commerce, National Institute of Standards and Technology, NISTIR 4315, 4316, and 4317 .

"The publishing of this collection of reports does not imply that the CALS Office has endorsed the conclusions or recommendations presented. 
An additional deliverable completed for CALS by the Graphics Software Group during FY89 detailed the impact that two other graphics standards (namely PHIGS, or the Programmers Hierarchical Interactive Graphics System, and PIK, or the Programming Imaging Kernel) ${ }^{5}$ will have on the CALS environment. It was published under separate cover, and is available through the CALS Policy office or through the National Technical Information Service.

${ }^{5}$ Kemmerer, Sharon J., and Skall, Mark W., "Graphics Application Programmer's Interface Standards and CALS," U.S. Department of Commerce, National Institute of standards and Technology, NISTIR 89-4199, October 1989. 


\section{CONTRIBUTORS}

NIST would like to acknowledge the major technical contributors to the separate reports contained herein. They are:

Peter R. Bono Associates, Inc., in particular Dr. Peter R. Bono, for his work on (1) above;

Henderson Software Company, in particular Mr. Lofton Henderson, for his work on (2) and (3) above, and

GSC Associates Inc., in particular Dr. George S. Carson, for his work on (4) above.

The editor would also like to gratefully acknowledge the efforts of those who participated in the review process of the documents presented in this report, namely:

David K. Jefferson

Sharon J. Kemmerer

Roy S. Morgan

Susan Quinn Sherrick

Lynne Rosenthal

Mark W. Skall 


FINAL REPORT

CALS FY89 SOW TASK 4.1.1

TEST REQUIREMENTS DOCUMENT FOR

CALS CGM CONFORMING BASIC METAFILES 


\section{TABLE OF CONTENTS}

I. OVERVIEW AND METHODOLOGY
A. Overview
B. Purpose
C. Scope
D. Use
E. Methodology

II. REFERENCE ARCHITECTURE FOR A CGM PARSER/VERIFIER • • • . 3

A. Overview . . . . . . . . . . . . . . . . . 3

B. Parser/Verifier Operation . . . . . . . . . . . 3

1. Step 1: Physical to Logical Mapping . . . . . 3

2. Step 2: Lexical Phase ............ 4

3. Step 3: Element Recognition . . . . . . . 4

4. Step 4: Element Processing . . . . . . . . 4

5. Step 5: Special Element Processing . . . . . 6

6. Step 6: Final Processing . . . . . . . . . 9

7. Step 7: Reporting ............ 10

III. REQUIREMENTS EXTRACTED FROM ISO 8632-1:1987 . . . . . . 11

A. Explanation of Contents . . . . . . . . . . . . 11

B. Organization of Requirements . . . . . . . . . . . 11

1. Required Elements . . . . . . . . . . . 12

2. Required Order of Elements . . . . . . . 12

3. Some Global Constraints on the content of the CGM . . . . . . . . . . . . . . . . 14

4. Metafile Defaults Replacement Requirements . . 16

5. Non-final Text Requirements . . . . . . . . 17

6. String Contents Requirements . . . . . . . 19

7. General Assertions about Parameter Values . . 20

8. Specific Parameter Range Constraints . . . . 21

IV. REQUIREMENTS EXTRACTED FROM ISO 8632-3:1987 . . . . . . 29

A. Explanation of Contents . . . . . . . . . . . 29

B. Organization of Requirements . . . . . . . . . 29

C. Errors in the CGM Specification . . . . . . . . 29

V. REQUIREMENTS EXTRACTED FROM MIL-D-28003 . . . . . . . 39

A. Explanation of Contents . . . . . . . . . . . 39

B. Organization of Requirements . . . . . . . . . 39

1. General CALS . . . . . . . . . . . . 40

2. Physical to Logical Mapping . . . . . . . 40

3. Metafile Structure and Ordering . . . . . . 41

4. Parameter Decoding . . . . . . . . . . . 42

5. Parameter Ranges . . . . . . . . . . . . 42

6. Size Constraints .. . . . . . . . . . . 47

7. GDP and ESCAPE Elements . . . . . . . . . 47

8. Colour/Pattern Usage Rules . . . . . . . 48

VI. CROSS REFERENCE TABLE BETWEEN REQUIREMENTS AND TESTING • 
TABLE OF CONTENTS (Continued)

APPENDIX A: CGM ELEMENT ORDERING REQUIREMENTS . . . . . . 61

APPENDIX B: CGM ELEMENT LENGTH REQUIREMENTS

FROM ISO 8632-3:1987 . . . . . . . . . 71

APPENDIX C: ALLOWABLE PARAMETER RANGES FOR

BINARY ENCODED CGM ELEMENTS . . . . . . 79 


\section{ABSTRACT}

The purpose of this report is to satisfy the requirements of CALS FY89 statement of Work Task 4.1.1, which states:

Continue to accelerate the development of CGM validation routines and ensure the input of CALS requirements.

In previous related tasks, NIST/NCSL has: developed a plan for the development of conformance tests for both FIPS PUB 128 (CGM) and MIL-D-28003; compared the variability of commercial CGM generator implementations and how well they conform to MIL-D-28003; and assessed the impact of CGM test development strategy with respect to the CALS environment and the marketplace. Finally, as part of last fiscal year's work on CALS, NIST/NCSL prepared a comprehensive list of tasks that must be accomplished in order to put into place a testing service for both FIPS PUB 128 and MIL-D-28003. This report is a follow-on to that work, and fulfills one of the most important tasks identified concerning the development of a Test Method, i.e. to create a Test Requirements Document. 



\section{A. Overview}

The Computer Graphics Metafile (CGM) standard (ISO 8632; ANSI/X3.122; FIPS 128) is a data transfer standard. In particular, it specifies the content of a logical file to be used in storing and interchanging picture descriptions among applications. Conformance statements in the CGM standard apply to instances of CGMs and not to generators (writers) of CGMs nor to interpreters (readers) of CGMs. Consequently, the standard specifies mainly syntactic requirements; there are very few semantic requirements to be met by a conforming instance of a CGM.

\section{B. Purpose}

The purpose of a Test Requirements document is to gather together all requirements that must be satisfied in order for a given instance of an implementation of a graphics standard to be in conformance with that standard. In the case of the CGM standard, an instance of a CGM whose conformance is in question will be called a CGM-under-test.

\section{Scope}

This test requirements document is meant to apply to CGMs that attempt to comply with MIL-D-28003, the CALS Application Profile for the CGM. Consequently, the document has gathered only those requirements that apply to Binary Encoded CGMs; i.e., those that conform to Parts 1 and 3 of the CGM standard.

\section{Use}

This document is intended for use by (1) programmers who must build a testing tool capable of determining whether a given instance of a CGM is in conformance with MIL-D-28003 and (2). buyers who need to judge, by whatever means are available, whether a given CGM is in conformance with MIL-D-28003. In addition, programmers responsible for producing products with capabilities for CGM generation or interpretation will find this document valuable.

Although specifically targeted at CALS-conforming CGMs, the document presents the CALS-specific requirements of MIL-D-28003 separately, so that the document is of value to anyone needing to test Binary Encoded CGMs for conformance to the CGM standard. In addition, because the Functional Requirements are documented separately from the Encoding Requirements, this document could also serve as the basis for a more comprehensive CGM Test Requirements document that deals with all three standardized encodings of the CGM. 


\section{E. Methodology}

The CGM Functional Description, ISO 8632-1:1987, was systematically read and all requirements relating to the conformance of instances of CGMs were extracted. Section III presents the results of that analysis. A similar approach was taken for the CGM Binary Encoding, ISO 8632-3:1987, and the CALS Application Profile, MIL-D-28003. The results are summarized in sections IV and V, respectively. In these sections, like requirements are grouped. [NOTE: Because this document is intended to be used in the development of an international CGM conformance testing tool, the requirements were derived from ISO 8632:1987. It should be further noted that ISo 8632 and ANSI X3.122 are identical except for document layout and style (i.e., page numbers may differ).]

From the total set of requirements, three major summary tables were assembled and are presented as Appendices. First, Appendix A contains the description of a finite state machine that enforces exactly all CGM Element ordering requirements contained in the standard. Next, Appendix B contains, in tabular form, the parameter list lengths for all elements in a CALS-conforming CGM. Finally, Appendix $C$ presents a matrix stating the requirements that must be met by each parameter of each CGM Element appearing in a CALS-conforming CGM.

These tables summarize and account for over two-thirds of the specific requirements statements found in the CGM standard. The conformance implications of the remaining requirements statements cannot fruitfully be characterized in a simple table. Instead, an expository technique has been chosen, which is elaborated in Section II, based on describing the behavior of a hypothetical "black box," whose task it is to determine whether a given CGMunder-test is in conformance with MIL-D-28003.

The black box is called a "CGM Parser/Verifier." Its operation is broken down into six major steps, which can be thought of as relatively autonomous program modules, plus a reporting step. Section II gives a complete description of the behavior of the parser/verifier and assumes that the parser/verifier uses the information embodied in the summary tables of Appendices A, B, and $C$.

To complete the report, section VI gives a cross reference between each raw requirement statement and the Table (Appendix $A$, $B$, or C) or step (in section II) where the test requirement is represented for testing. 
II. REFERENCE ARCHITECTURE FOR A CGM PARSER/VERIFIER

\section{A. Overview}

The CGM standard, ISO 8632:1987, Parts 1 (Functional Description) and 3 (Binary Encoding), specifies the content and structure of a conforming metafile. This section describes the high-level design of a CGM parser/verifier whose objective is to determine whether a given CGM is conforming. If the CGM-under-test is conforming, the parser/verifier should produce a conformance report stating this fact. If the CGM-under-test does not conform to the standard, the conformance report should include a description of each conformance violation discovered including its location (as a logical octet offset from the start of the CGM) .

MIL-D-28003, "Digital Representation for Communication of Illustration Data: CGM Application Profile," places additional constraints on the structure and content of a CALS basic conforming metafile. In the following description of the parser/verifier operation, CALS-specific tests are separated into their own paragraphs and preceded with the phrase "CALS".

\section{B. Parser/Verifier Operation}

The parser/verifier is divided into a number of relatively autonomous modules described in the sections that follow.

\section{Step 1: Physical to Logical Mapping}

The CGM-under-test is opened and the initial information is read from the file into an internal buffer. Because the CGM is logically viewed as a continuous stream of bits, organized as octets and words, according to specific definitions in the standard, it is in this module that all operating-system and programming-language-specific matters like byte swapping and fixed-length and variable-length record structures are dealt with. For the rest of this discussion, it is assumed that all subsequent steps are able to get any number of octets from the logical CGM-under-test in the correct order without worrying about whether there are file markers, record markers, and the like embedded in the stream of octets returned to the lexical phase (step 2).

CALS. MIL-D-28003 specifies that basic conforming metafiles consist of 80-octet fixed-length records. If transmitted on magnetic tape in accordance with MIL-STD 1840A, they are blocked into 800-octet physical records (i.e., 10 records per block). 


\section{Step 2: Lexical Phase}

Two octets are obtained from the CGM-under-test. They represent the command header (short form). The element class and id are extracted and saved. The length is examined to determine if a long-form element is present. If so, another two octets are obtained. The parameter length information (short or long) is saved. Bit 15 is examined in the long form to determine whether data partitioning is in effect. The starting point of the next command header (or next data partition) is calculated, taking into consideration that command headers and partitions start on word boundaries.

One special piece of processing that can occur here is detecting when all the elements comprising the parameter data in a METAFILE DEFAULTS REPLACEMENT element have been picked up. Prior to getting the command header of the first element following the METAFILE DEFAULTS REPLACEMENT element, the state should be set to MDOP.

CALS. In a basic conforming metafile, the METAFILE DEFAULTS REPLACEMENT element shall not be partitioned. If partitioning is encountered, generate an appropriate CALS-conformance violation message, write it to the profile error report file, and increment the count of such errors.

\section{Step 3: Element Recognition}

If the class/id is recognized as one of the valid opcodes, a frequency count for each opcode encountered is incremented. Then, processing passes to step 4 (Element Processing).

Otherwise, a conformance violation message is formulated and output to an error report file, and the error count for this category of error is incremented. Then the octet pointer into the CGM-under-test is positioned to the start of the next command header, skipping past all parameter data for the illegal element, including any data partitions that might be encountered. Control is returned to step 2 .

\section{Step 4: Element Processing}

If this element is the first element in the metafile and it is not the BEGIN METAFILE element, the parser/verifier immediately halts processing with an appropriate message.

Each valid CGM element needs its own processing section, because different specific actions need to be taken for each element. However, the processing follows a familiar pattern, which is described in the following paragraphs. 
It is assumed that the parser/verifier has established a set of global variables which contain both the current metafile default values for all Metafile Descriptor, Picture Descriptor, Control, and Attribute elements and the current parser/verifier values for all Picture Descriptor, Control, and Attribute elements. It is also assumed that the parser/verifier is checking element ordering rules by implementing the finite-state machine logic according to the description provided in Appendix $A$.

(Step 4A: Check Element order) First, the current state of the parser/verifier is compared against the states allowed for this element. If the element is not allowed in this state, an appropriate error message is formulated and written and the error count updated for this class of error. However, processing is allowed to continue.

CAIS. MIL-D-28003 places some constraints on the use of ESCAPE elements, and GDP elements are not permitted. These constraints are shown in the state table summary section (Appendix A).

(Step 4B: Acquire Parameter) The correct number of octets for each parameter is picked up in turn from the CGM-under-test. This number often varies according to the current variable settings. If the number of octets needed would exceed the number of octets remaining available for this element, processing of this element is aborted and control passes to step 2, after an appropriate error message is formulated and written and the error count updated for this class of error. During this step, one must be careful to observe rules relating to byte and word alignment, where they apply (e.g, in the CELL ARRAY element)-see section IV, requirements B2, B13, B14, B15, B18, B19, B32, and $\mathrm{B} 33$.

CAIS. MIL-D-28003 restricts the range of allowed precisions to a subset of all those allowed by the CGM standard. The length (in octets) of each element in a basic conforming metafile is shown in a summary table (Appendix B). All valid element lengths are shown where the basic set permits more than one precision.

(Step 4C: Decode Parameter) The parameter is decoded according to the data type expected for this element. If any error occurs upon decoding, an appropriate error message is formulated and written and the error count updated for this class of error. 
(Step 4D: Check Parameter Range) The parameter is then checked against any range constraints that might apply. These constraints might be universal (e.g., an enumerated type must be either 0 or 1 ) or might be dependent upon the current variable settings (e.g., all colour indices must be non- negative and no greater than the maximum colour index). If any parameter fails a range check, an appropriate error message is formulated and written and the error count updated for this class of error. Range checks for strings include verifying that the string length is non-negative and that the strings contain only legal character codes. This latter check may depend upon the value of the Character Coding Announcer.

CAIS. MIL-D-28003 permits only a subset of the permissible CGM values for each parameter to be present in a basic conforming metafile. Appendix $C$ shows the allowable range for each parameter and is annotated with these additional CALS- specific range constraints, when the CALS Basic set is a proper subset of the permissible CGM values according to the CGM standard.

(Step 4E: Perform Element-specific Special Processing) once all the parameters for an element have been acquired, any special processing for that element can take place. Special processing for each element or group of elements is described in step 5.

\section{Step 5: Special Element Processing}

BEGIN METAFIIE: Set all current metafile defaults to the fixed defaults specified in the standard. Save the string parameter contents. Set state to MDOP.

END METAFILE: Set state to MFCL. Go to step 6 (Final Processing).

BEGIN PICTURE: Reset all current picture descriptor variables to the corresponding values for the current metafile defaults. Save the contents of the string parameter of each picture. Set state to PDOP. At the first BEGIN PICTURE, examine the frequency count data to verify that the METAFILE VERSION and METAFILE ELEMENT LIST elements were present in the Metafile Descriptor.

BEGIN PICTURE BODY: Set state to PBOP.

END PICTURE: Set state to PICL.

CAIS. Perform the Colour/Pattern Usage checks described in the following: 
The colour index used/set/redefined information and the pattern index used/set/redefined information shall be compared with the requirements of MIL-D-28003. CALS conformance violation messages shall be generated, reported, and counted, under the following circumstances:

(a) Not all colour indexes used were set, unless none of the indexes were set.

(b) The colour redefinition list is non-empty.

(c) The pattern redefinition list is non-empty.

Metafile Descriptor Elements (general): Set the corresponding value in the current metafile defaults global data structure.

METAFILE DESCRIPTION: Save the contents of the string parameter.

CALS. Verify that the description contains "MIL-D-28003/BASICI" as a substring. Verify that some additional text is present-text that could serve to identify the company or product.

METAFILE ELEMENTS LIST: Mark the element frequency count global data structure with the opcodes in the list. Correctly expand the codes for "drawing set" and "drawing plus controls set".

METAFILE DEFAULTS REPLACEMENT: Set state to MMDR. Set octet counter (file offset) to correct value so that state can be set back to MDOP when all the parameters for this element have been processed (see step 2 discussion).

CALS. FONT LIST: All the font names encountered in the font list must match one of the sixteen Hershey font names specified in MIL-D-28003.

Picture Descriptor Elements (general): Set the corresponding value in the current variables global data structure if the state is PDOP and in the current metafile defaults global data structure if the state is MMDR.

CAIS. BACKGROUND COLOUR. FOllow the same processing as required for colour table elements.

Control Elements (general): Set the corresponding value in the current variables global data structure if the state is PBOP and in the current metafile defaults global data structure if the state is MMDR. 
Geometric Primitives (general): check for any special constraints on the number of entities expected. For example, check for at least 2-point polylines, 3-point polygons, and disjoint polylines with an even number of points.

CAIS. GDP: Report an error, because no GDPs are registered as yet.

CAIS. The number of colour values shall not exceed $1,048,576$ in a CELL ARRAY element. The number of points in any metafile element shall not exceed 1024. No string parameter, with the exception of data records, shall exceed 254 characters in length; data records shall not exceed 32767 characters.

CAIs. If the default colour index for this type of primitive is the current colour index, mark the corresponding internal colour table entry as used. If the entry is not already marked as set, then generate, record, and count a CALS Application Profile conformance violation if any other index has already been set.

CAIS. If this is a fill-area type primitive, if the current interior style is "pattern," and if the default pattern index is the current pattern index, mark the corresponding internal pattern table entry as used. If the entry is not already marked as set, then generate, record, and count a CALS Application Profile conformance advisory (which may become a violation in a future version of MIL-D-28003).

"not final" TEXT and RESTRICTED TEXT: set state to TXOP. "final" APPEND TEXT: set state to PBOP.

Attribute Elements (general): Set the corresponding value in the current variables global data structure, if the state is PBOP, and in the current metafile defaults global data structure, if the state is MMDR.

CAIS. The number of colour values shall not exceed 2048 in a pattern table and 256 in a COLOUR TABLE element. No string parameter, with the exception of data records, shall exceed 254 characters in length; data records shall not exceed 32767 characters.

CAIS. Colour Value selection Elements (for indexed colour): These elements include IINE COLOUR, MARKER COLOUR, TEXT COLOUR, FILL COLOUR, and EDGE COLOUR. Also included are the colour index aspects of the corresponding bundles, when the appropriate aspect source flag controlling the setting of colour is individual. Mark the corresponding internal colour table entry as used. If the entry is not already marked as set, record and count a CALS Application profile conformance violation if any other index has 
already been set. This processing does not take place if the parser/verifier is in the MMDR state (that is, if default colour indexes are being specified).

CALS. PATTERN INDEX: Mark the corresponding internal pattern table entry as used. If the entry is not already marked as set, generate, record, and count a CALS Application Profile conformance advisory (which may become a violation in a future version of MIL-D-28003). This processing does not take place if the parser/verifier is in the MMDR state (that is, if a default pattern index is being specified).

CAIS. PATTERN TABLE: Save the pattern in the internal pattern table. Mark the entry as "set." If the entry is already marked as used and the pattern table parameter values are different from the values already set in the internal table, record this pattern index as "redefined". This processing does not take place if the parser/verifier is in the MMDR state (that is, if a default pattern table is being specified). Note also that no redefinition of pattern table entries is allowed once the first primitive has been encountered by the parser.

CALS. COLOUR TABLE: Save the colour values in the internal colour table. Mark the entry as "set." If the entry is already marked as used and the colour table parameter values are different from the values already set in the internal table, record this colour index as "redefined". This processing does not take place if the parser/verifier is in the MMDR state (that is, if a default colour table is being specified). Note also that no redefinition of colour table entries is allowed once the first primitive has been encountered by the parser.

CALS. ESCAPE: Report an error if the id is not $-301,-302$, or 303, because these are the only ESCAPEs authorized for use for CALS.

\section{Step 6: Final Processing}

Once the entire CGM-under-test has been interpreted, there are still two global checks that remain to be accomplished.

(Step 6A: Required Elements) The frequency count data is examined to verify that END METAFILE has occurred.

(Step 6B: Correctness of METAFILE ELEMENT LIST) The frequency count data is compared with the elements marked as a result of their presence in the METAFILE ELEMENT LIST to verify that every element actually present in the metafile was mentioned in the METAFILE ELEMENT LIST. 


\section{Step 7: Reporting}

A FIPS PUB 128 conformance report is produced. The report should contain, at a minimum:

(a) The file name of the CGM-under-test.

(b) The (logical) size of the file in octets.

(c) The contents of the string parameters associated with BEGIN METAFILE and METAFILE DESCRIPTION (if present).

(d) A count of the number of pictures present and the starting octet count and content of the string parameter associated with each BEGIN PICTURE.

(e) A statement of conformance reporting the total number of elements tested and the number and type of errors found (if any).

(f) Specific error messages for each conformance violation detected along with information that permits localization of the error (e.g., element name and offset into the file).

CALS. A MIL-D-28003 conformance report supplement should be appended to the basic conformance report. It should provide:

(a) A statement of CALS conformance reporting the number and type of Application Profile errors found (if any).

(b) Specific error messages for each CALS CGM Application profile conformance violation detected along with information that permits localization of the error (e.g., element name and offset into the file). 


\section{A. Explanation of Contents}

The CGM Functional Description, ISO 8632-1:1987, was systematically read and all requirements relating to the conformance of instances of CGMs were extracted. Each separate statement of requirements is assigned a number and quoted. The requirement numbers are assigned sequentially from one and all start with the letter "F," indicating that these requirements come from the CGM Functional Description, Part 1. A suffix-"a," "b," "c"--is added if the identical requirement is stated multiple times in different places.

A citation for each statement (shown in boldface) is given according to the following scheme: The Part 1 clause number is provided on the first line; on the second line, for clause 4 and Clause 6 citations, the paragraph number (p) and sentence number(s) are specified as "\#p/s", followed by the page number on which the text occurs. For clause 5 citations, the only difference is that the second line starts with either a "P" or "D", rather than a "\#". "P" means that the information is contained in the Parameters section of the clause and "D" that the information is contained in the Description section of the clause.

Any commentary on the requirements statement is shown as a note enclosed in brackets ([NOTE:...]).

\section{B. Organization of Requirements}

The requirements are grouped into eight main categories. Within each category, the requirements are generally stated in order of their clause number. The only exception to this rule is when a requirement is stated in several places in the standard. These requirement statements are grouped together.

The eight categories of requirements statements are:

Required Elements

Required Order of Elements

Some Global Constraints on the content of the CGM

Metafile Defaults Replacement Requirements

Non-final Text Requirements

String Contents Requirements

General Assertions about Parameter Values

Specific Parameter Range constraints 


\section{Required Elements}

F1: Clause 4.1

$\# 4 / 1 \quad$ p. 9

A minimal correct metafile consists of BEGIN METAFILE, a Metafile Descriptor consisting of METAFILE VERSION and METAFILE ELEMENT LIST, and END METAFILE.

F2: Clause 5.2 .1

D1/3 p.44

BEGIN METAFILE shall occur exactly once in a metafile.

F3: Clause 5.3.1

D $1 / 2 \quad$ p. 47

[The METAFILE VERSION] element shall occur in the Metafile Descriptor of every metafile.

F4: Clause 5.3.11

$\mathrm{D} 1 / 3 \quad$ P. 50

METAFILE ELEMENT LIST shall occur in the Metafile Descriptor of every metafile.

2. Required order of Elements

F5: Clause 4.2

$\# 1 / 1 \quad$ p. 9

Every metafile starts with a BEGIN METAFILE element ...

F6: Clause 5.2.1

D1/1 p.44

[BEGIN METAFILE] is the first element of a metafile.

F7: Clause 4.2

\#1/1 p. 9

Every metafile... ends with an END METAFILE element.

F8: Clause 5.2.2

D1/1 p.44

[END METAFILE] is the last element of a metafile. 
F9: Clause 4.3

$\# 2 / 3 \quad$ p. 10

The CGM contains a single Metafile Descriptor ... [which] immediately follows the BEGIN METAFILE element in a metafile (with the possible exception of intervening external and escape elements).

[NOTE: This implies that Metafile Descriptor elements may appear only before the first BEGIN PICTURE element in the metafile, if the metafile contains any pictures.]

F10: Clause 4.3

$\# 2 / 4 \quad$ p. 10

External and escape elements may appear anywhere between the BEGIN METAFILE element and first BEGIN PICTURE element (if one exists) and after the last END PICTURE element (if one exists) and the END METAFILE element.

F11: Clause 4.4

$\# 1 / 3 \quad$ p. 12

If included in a picture, [Picture Descriptor] elements shall appear after the BEGIN PICTURE element and before the BEGIN PICTURE BODY element.

F12: Clause 5.4.1

D1/5 p.56

If used, SCALING MODE shall appear in the Picture Descriptor.

F13: Clause 5.4.2

D2/4 p.56

If used, COLOUR SELECTION MODE shall appear in the Picture Descriptor.

F14: Clause $5.4 \cdot 3$

D2/3 p.57

If used, LINE WIDTH SPECIFICATION MODE shall appear in the Picture Descriptor.

F15: Clause 5.4.4

D2/3 p. 57

If used, MARKER SIZE SPECIFICATION MODE shall appear in the Picture Descriptor. 
F16: Clause 5.4 .5

D2/3 P. 57

If used, EDGE WIDTH SPECIFICATION MODE shall appear in the Picture Descriptor.

F17: Clause 4.4

$\# 1 / 4 \quad$ p.12

Escape and external elements are permitted in the Picture Descriptor.

F18: Clause 4.5
$\# 1 / 2$
p. 14

Control elements ... may appear in the picture bodies in the metafile.

F19: Clause 4.9

$\# 1 / 1 \quad$ p.39

External elements ... may appear anywhere in the CGM.

F20: Clause 4.10

\#Fig. 12 p.41

Control, Graphical Primitive, and Attribute elements can appear only while a picture is "open," that is, between a BEGIN PICTURE BODY element and the next END PICTURE element.

F21: Clause 5.2 .5

D2/1 P. 46

Only external and escape elements may occur between END PICTURE and BEGIN PICTURE or between END PICTURE and END METAFILE.

\section{Some Global Constraints on the content of the CGM}

F22: Clause 4.3

$\# 2 / 1 \quad$ p. 10

The METAFILE ELEMENT LIST lists at least those standardized elements that occur in the metafile.

223: Clause 5.3.11

D1/1 P. 50

All of the elements that may be encountered in the metafile and that are not mandatory are listed [in the METAFILE ELEMENT LIST]. 
F24: Clause 4.7 .7

$\# 5 / 2 \quad$ p. 38

There is a Metafile Descriptor element, COLOUR VALUE EXTENT, which allows metafile generators to specify the minimum and maximum metafile colour values.

[NOTE: This requirement implies that all colour values should be checked to ensure that they lie in the range specified in the COLOUR VALUE EXTENT.]

F25: Clause 5.3.10
D1/1-2
p. 49

[For COLOUR VALUE EXTENT] the parameters represent an extent which bounds the direct colour values that will be encountered in the metafile. It need not represent the exact extent of colour values contained in the metafile.

F26: Clause 5.3.9

D1/1 p. 49

[FOr MAXIMUM COLOUR INDEX] the parameter represents an upper bound (not necessarily the least upper bound) on colour index values that will be encountered in the metafile.

[NOTE: Therefore, the value of this parameter should be greater than or equal to all colour indices found in the metafile.]

F27: Clause 5.4.2

D2/1 P. 56

Only one colour mode may be used within a picture.

F28: Clause 5.4.3

D2/1 P.57

Only one line width mode may be used within a picture.

F29: Clause 5.4.4

D2/1 p.57

only one marker size mode may be used within a picture.

F30: Clause 5.4.5

D2/1 p.57

only one edge width mode may be used within a picture. 


\section{Metafile Defaults Replacement Requirements}

F31: Clause 4.4.4

$\# 3 / 1 \quad$ p. 12

The default state of the [VDC] extent ... can be changed in the METAFILE DEFAULTS REPLACEMENT element in the Metafile Descriptor.

F32: Clause 4.4.5

$\# 1 / 3 \quad$ p.14

The default background colour [can be] specified in the METAFILE DEFAULTS REPLACEMENT element.

F33: Clause 5.3.12

$\mathrm{P} 1 / 1 \quad$ P. 50

Picture Descriptor, Control, and Attribute elements [may appear in the METAFILE DEFAULTS REPLACEMENT element].

F34: Clause 5.3.12

D1/4 p. 50

Any subset of the elements given defaults in clause 6 may be included [in the METAFILE DEFAULTS REPLACEMENT element].

F35: Clause 5.3 .12

D2/3 p.50-1

An element [can] occur more than once in the default replacement list. 
F36: Clause 6

\#al1 p.101-103

By implication (all non-Metafile-Descriptor elements mentioned in Clause 6), any of the following elements may appear in a METAFILE ELEMENTS REPLACEMENT element:

SCALING MODE

COLOUR SELECTION MODE

LINE WIDTH SPECIFICATION MODE

MARKER SIZE SPECIFICATION MODE

EDGE WIDTH SPECIFICATION MODE

VDC EXTENT

BACKGROUND COLOUR

VDC INTEGER PRECISION

VDC REAL PRECISION

AUXILIARY COLOUR

TRANSPARENCY

CLIP RECTANGLE

CLIP INDICATOR

LINE BUNDLE INDEX

LINE TYPE

LINE WIDTH

LINE COLOUR

MARKER BUNDLE INDEX

MARKER TYPE

MARKER SIZE

MARKER COLOUR

TEXT BUNDLE INDEX

TEXT FONT INDEX

TEXT PRECISION

\author{
CHARACTER EXPANSION FACTOR \\ CHARACTER SPACING \\ TEXT COLOUR \\ CHARACTER HEIGHT \\ CHARACTER ORIENTATION \\ TEXT PATH \\ TEXT ALIGNMENT \\ CHARACTER SET INDEX \\ ALTERNATE CHARACTER SET INDEX \\ FILL BUNDLE INDEX \\ INTERIOR STYLE \\ FILI COLOUR \\ HATCH INDEX \\ PATTERN INDEX \\ EDGE BUNDLE INDEX \\ EDGE TYPE \\ EDGE WIDTH \\ EDGE COLOUR \\ EDGE VISIBILITY \\ FILL REFERENCE POINT \\ PATTERN TABLE \\ COLOUR TABLE \\ ASPECT SOURCE FLAGS
}

\section{Non-final Text Requirements}

F37: Clause 4.6.3.2

$\# 2 / 1 \quad$ p.17

Changes to the text attributes TEXT FONT INDEX, CHARACTER EXPANSION FACTOR, CHARACTER SPACING, TEXT COLOUR, CHARACTER HEIGHT, CHARACTER SET INDEX, ALTERNATE CHARACTER SET INDEX, and TEXT BUNDLE INDEX, and to the control elements AUXILIARY COLOUR and TRANSPARENCY are permitted between a non-final text element and its succeeding APPEND TEXT element.

[NOTE: NB: Clause 4.7 .6 and Figure 12 (p. 41) also indicates that TEXT PRECISION is okay, while the formal grammar (which is not part of the CGM standard) allows CHARACTER ORIENTATION, which is not mentioned in Clause 4.7 .6 nor in Figure 12.] 
F38: Clause $4 \cdot 6 \cdot 3 \cdot 3$

$\# 1 / 4 \quad$ p. 17

The initial [text] element is always TEXT or RESTRICTED TEXT; subsequent elements may only be APPEND TEXT.

F39: Clause 4.7.6

\#3/1 p. 24

The attributes in the character representation and placement group [TEXT FONT INDEX, CHARACTER SET INDEX, ALTERNATE CHARACTER SET INDEX, TEXT PRECISION, CHARACTER EXPANSION FACTOR, CHARACTER SPACING, TEXT COLOUR, CHARACTER HEIGHT, AUXILIARY COLOUR, TRANSPARENCY] and TEXT BUNDLE INDEX maY be changed within a string.

F40: Clause 4.10

\#Fig. 12 p. 41

No elements, other than the following list of elements, may appear between a "not final" TEXT or RESTRICTED TEXT element and a subsequent "final" APPEND TEXT element: "not final" APPEND TEXT, TEXT FONT INDEX, TEXT PRECISION, CHARACTER EXPANSION FACTOR, CHARACTER SPACING, TEXT COLOUR, CHARACTER HEIGHT, CHARACTER SET INDEX, ALTERNATE CHARACTER SET INDEX, TEXT BUNDLE INDEX, AUXILIARY COLOUR, TRANSPARENCY.

F41a: Clause 5.6 .4

D3/1 p.63

also

F41b: Clause 5.6 .5

D6/1 p.64

also

F41C: Clause 5.6 .6

D3/1 p. 65

The flag parameter is used to permit changing the following text attributes and control elements within a string which will be aligned as a single block: TEXT FONT INDEX, TEXT PRECISION, CHARACTER EXPANSION FACTOR, CHARACTER SPACING, TEXT COLOUR, CHARACTER HEIGHT, CHARACTER SET INDEX, ALTERNATE CHARACTER SET INDEX, TEXT BUNDLE INDEX, AUXILIARY COLOUR, and TRANSPARENCY. 
F42a: Clause 5.6.4

D4/1-3 p. 63

also

F42b: Clause 5.6.5

D7/1-3 p. 64

also

F42c: Clause 5.6 .6

D4/1-3 p. 65

If the flag is set to 'not final',... only the attribute setting elements listed above are allowed between this element and the APPEND TEXT element. With the exception of the ESCAPE element, no other metafile elements of any type are allowed.

\section{String Contents Requirements}

F43a: Clause 5.6 .4

D1/3 p. 63

also

F43b: Clause 5.6 .5

D2/3 p. 64

also

F43c: Clause 5.6 .6

D1/4 p.65

Format effector control characters (such as CR, LF, BS, HT, VT, and FF) are permitted in a [TEXT] string but their interpretation is implementation-dependent. 
F44a: Clause 5.6.4

D1/4 p. 63

also

F44b: Clause 5.6.5

D2/4 P. 64

also

F44C: Clause 5.6.6

D1 $/ 5 \quad$ P. 65

Control characters used for character set invocation and designation (SI, SO, ESC, SS2, and SS3) are permitted according to the setting of CHARACTER CODING ANNOUNCER.

F45: Clause 5.7.20

D3/1 P. 89

If the appropriate CHARACTER CODING ANNOUNCER is selected, the sO and SI controls and ISO 2022 escape sequences may be embedded within the string parameters of text elements.

\section{General Assertions about Parameter Values}

F46: Clause 4.3 .2

$\# 1 / 3 \quad$ p. 10

Two shorthand names for CGM elements are also provided for use with the METAFILE ELEMENT LIST [element].

F47: Clause 4.3.2.1

\#2/1 p.10

The drawing set includes a set of listed elements.

F48: Clause 4.3.2.2

\#2/1 p.11

The drawing plus control set includes a set of listed elements.

F49: Clause 4.6 .5

\#2/1 p.19

The colour values [of a CELL ARRAY element] are either direct colour values or indexes into the COLOUR TABLE, according to the current COLOUR SELECTION MODE. 
F50: Clause 5.4.2

D2/3 P.56

All occurrences of colour-setting elements (AUXILIARY COLOUR, LINE COLOUR, MARKER COLOUR, FILL COLOUR, EDGE COLOUR, TEXT COLOUR) as well as the colour lists of CELL ARRAY and PATTERN TABLE shall be in the current [colour selection] mode.

F51: Clause 4.6 .5

\#2/2 p.19

The colour values [of a CELL ARRAY element] are in the precision declared by a 'local colour precision' parameter of the CELL ARRAY element.

F52: Clause 5.4.6

D6/1 p.58

Specification of [VDC] values outside VDC EXTENT in parameters of CGM elements is permitted.

F53: Clause 5.6.9

D3/4-5 P. 69

If the picture uses indexed colour selection, then the form of the [local colour precision CELL ARRAY] parameter is the same as that of COLOUR INDEX PRECISION. If the picture uses direct colour selection, then the form of the parameter is the same as that of COLOUR PRECISION.

F54: Clause $5 \cdot 6.32$

D3/4-5 P.96

If the picture uses indexed colour selection, then the form of the [local colour precision PATTERN TABLE] parameter is the same as that of COLOUR INDEX PRECISION. If the picture uses direct colour selection, then the form of the parameter is the same as that of COLOUR PRECISION.

\section{Specific Parameter Range Constraints}

F55: Clause 4.11
$2 / 1-2$
p. 40

Applications therefore shall not use parameter values in the reserved ranges for implementation or private use. Those metafile elements that will be affected by registration of graphical items are: LINE TYPE, MARKER TYPE, HATCH STYLE, EDGE TYPE, FONT LIST, GENERALIZED DRAWING PRIMITIVE, ESCAPE. 
F56: Clause 5.1

$\# 11 / 4$ p.43

Non-negative values [of type IX parameters] are reserved for (future) standardization.

F57: Clause 5.3.1

D2/1 P. 47

This version of the CGM standard is version one (1).

[NOTE: This implies that the value of P1 must always be "1".]

F58: Clause 5.6.1

D1/1 P. 62

[For POLYLINE] a line is drawn from ... the next-to-last point to the last point.

[NOTE: This might be taken to imply that the number of points must be at least 2.]

F59: Clause 5.6.2

D1/1 p.62

[For DISJOINT POLYLINE], a line is drawn from the starting point to the second point...

[NOTE: This might be taken to imply that the number of points must be at least 2. Also, that there should be an even number of points in the point list.]

F60a: Clause 5.6.4

D5/3 p.63

also

F60b: Clause 5.6 .5

D8/3 P. 64

also

F60c: Clause 5.6.6

D5/2 p.65

Text elements with a null string parameter are legal. 
F61: Clause 5.6 .9

D1/1 p. 69

In the general case, $P, Q$, and $R$ [--the first three parameters of CELL ARRAY--] can delimit an arbitrary parallelogram.

[NOTE: This implies that the area specified by the vertices $P$, $Q$, and $R$ should not be zero.]

F62: Clause 5.6.9

D4/1-2 p. 69

Legal values of the 'local colour precision' include the legal values of COLOUR (INDEX) PRECISION. In addition, each encoding defines a special value, the 'default colour precision indicator', as an indicator that the colour specifiers of the [CELI ARRAY] element are to be encoded in the coloUR (INDEX) PRECISION of the metafile; i.e., to indicate that the 'local colour precision' defaults to COLOUR (INDEX) PRECISION.

F63: Clause 5.6.10

D2/1 p. 71

Non-negative values of the [GDP] identifier are reserved for registration and future standardization and negative values are available for private use.

[NOTE: This implies that 0 is not a legal GDP identifier at this time.]

F64: Clause 5.6 .12

D2/1 p. 72

Valid values of [a CIRCLE element's] radius are non-negative VDC.

F65: Clause 5.6.15

D5/1 p. 75

Valid values of [a CIRCULAR ARC CENTRE element's] vector components are those which produce vectors of non-zero length.

F66: Clause 5.6 .15

D6/1 P. 75

Valid values of [a CIRCULAR ARC CENTRE element's] radius are nonnegative VDC. 
F67: Clause 5.6.16

D7/1 P. 76

Valid values of [a CIRCULAR ARC CENTRE CLOSE element's] vector components are those which produce vectors of non-zero length.

F68: Clause 5.6.16

D8/1 p.76

Valid values of [a CIRCULAR ARC CENTRE CLOSE element's] radius are non-negative VDC.

F69: Clause 5.6.17

D3/1 P. 76

Valid values of the three specifying points of the [ELLIPSE element] are those which yield three distinct points.

F70: Clause 5.6.18

D6/1 P.77

Valid values of the three specifying points of the [ELLIPTICAL ARC element] are those which yield three distinct points.

F71: Clause 5.6.18

D7/1 p.77

Valid values of [a ELLIPTICAL ARC element's] vector components are those which produce vectors of non-zero length.

F72: Clause 5.6.19

D7/1 p.78

Valid values of the three specifying points of the [ELLIPTICAL ARC CLOSE element] are those which yield three distinct points.

F73: Clause 5.6.19

D8/1 p.78

Valid values of [a ELLIPTICAL ARC CLOSE element's] vector components are those which produce vectors of non-zero length.

F74: Clause 5.7.1

D3/1 P.79

Legal values [of line bundle index] are positive integers. 
F75: Clause 5.7.2

D5/1 p. 79

Values [of line type] above 5 are reserved for registration and future standardization.

F76: Clause 5.7.3

D4/1 p. 80

Valid values of 'Iine width specifier' are non-negative VDC if LINE WIDTH SPECIFICATION MODE is 'absolute' and non-negative reals if LINE WIDTH SPECIFICATION MODE is 'scaled.'

F77: Clause 5.7.5

D3/1 p.81

Legal values [of marker bundle index] are positive integers.

F78: Clause 5.7.5

D6/1 p.81

Values [of marker type] above 5 are reserved for registration and future standardization.

F79: Clause 5.7.7

D4/1 p.82

Valid values of 'marker size specifier' are non-negative VDC if MARKER SIZE SPECIFICATION MODE is 'absolute' and non- negative reals if MARKER SIZE SPECIFICATION MODE is 'scaled.'

F80: Clause 5.7.9

D3/1 P.83

Legal values [of text bundle index] are positive integers.

F81: Clause 5.7.10

D4/1 p.84

Legal values of the font index parameter are positive integers.

F82: Clause 5.7.12

D5/1 p.85

Legal values of the character expansion factor are non- negative reals. 
F83: Clause 5.7.15
D3/1
p. 87

Valid values of 'character height' are non-negative VDC.

F84: Clause 5.7.16

D2/1 p.87

Valid values of the [character up and character base] vectors include any which have non-zero length and are not collinear.

F85: Clause 5.7.19

D2/1 p.89

Legal values of [the] character set index parameter are positive integers.

F86: Clause 5.7.20

D2/1 P. 89

Legal values of the alternate character set index parameter are positive integers.

F87: Clause 5.7.21

D2/1 P.90

Legal values of FILL BUNDLE INDEX are positive integers.

F88: Clause 5.7 .22

D2/1 p. 90

If other non-standardized values of interior style are used, they shall be given private values.

[NOTE: It is assumed that "private" in this context means "negative valued."]

F89: Clause 5.7 .24

D7/1 p.91

Values [of hatch index] above 6 are reserved for registration and future standardization.

F90: Clause 5.7 .25

D5/1 P.92

Legal values of PATTERN INDEX are positive integers. 
F91: Clause 5.7 .26

D2/1 p. 92

Legal values of EDGE BUNDLE INDEX are positive integers.

F92: Clause 5.7 .27

D5/1 p. 93

Non-negative values of the [edge type] index [above 5] are reserved for [registration and] future standardization.

F93: Clause 5.7 .28

D4/1 p. 94

Valid values of 'edge width specifier' are non-negative VDC if EDGE WIDTH SPECIFICATION MODE is 'absolute' and non-negative reals if EDGE WIDTH SPECIFICATION MODE is 'scaled.'

F94: Clause 5.7 .32

D2/1 p. 96

Legal values of the pattern table index parameter are positive integers.

F95: Clause 5.6 .32

D4/1-2 p. 96

Legal values of the 'local colour precision' include the legal values of COLOUR (INDEX) PRECISION. In addition, each encoding defines a special value, the 'default colour precision indicator', as an indicator that the colour specifiers of the [PATTERN TABLE] element axe to be encoded in the COLOUR (INDEX) PRECISION of the metafile; i.e., to indicate that the 'local colour precision' defaults to COLOUR (INDEX) PRECISION.

F96: Clause 5.7 .33

D3/2 p. 96

The pattern size vectors ... define a parallelogram.

[NOTE: This might be taken to imply that the vectors have nonzero length and are not collinear.]

F97: Clause 5.7 .34

D2/1 p.97

Legal values of the colour index are non-negative integers. 
F98: Clause 5.8.1

D1/3 P. 99

Non-negative values [of the ESCAPE function identifier] are reserved for registration and future standardization.

[NOTE: This implies that 0 is not a valid identifier at this time.] 


\section{A. Explanation of Contents}

The CGM Binary Encoding, ISO 8632-3:1987, was systematically read and all requirements relating to the conformance of instances of binary-encoded CGMs were extracted. Each separate statement of requirements is assigned a number and quoted. The requirement numbers are assigned sequentially from one and all start with the letter "B," indicating that these requirements come from the CGM Binary Encoding, Part 3 .

A citation for each statement (shown in boldface) is given according to the following scheme: The part 1 clause number is provided on the first line; on the second line, for clause 4, 5, and 9 citations, the paragraph number (p) and sentence number(s) are specified as "\#p/s", followed by the page number on which the text occurs. For clause 6 citations, the only difference is that the second line starts with a Note number, rather than a "\#". This means that the information is contained in a note on the specified page. Similarly, for clause 7 citations, the only difference is that the second line starts with a code number, rather than a "\#". This means that the information is contained on the specified page in a note which expands upon the information in the tables in clause 7 .

Any commentary on the requirements statement is shown as a note enclosed in brackets ([NOTE:...]).

\section{B. Organization of Requirements}

The requirements are stated in order of their clause number.

\section{c. Errors in the CGM Specification}

Two errors were noted in Iso $8632-3: 1987$ :

(a) Clause 7.3 Table 4 (p.21): The parameter range for the first METAFILE ELEMENT LIST parameter should be +IR not $++I R$.

(b) Clause 7.3 Table 8 (p.32): The parameter range for CHARACTER EXPANSION FACTOR should be $++R R$ (not $+R R$ ) to be consistent with the other Parts of the CGM standard. However, it should be noted that $++R R$ is consistent with GKS (ISO 7942) and the other Parts of the CGM standard are not! 
B1: Clause 4.3

$\# 1 / 1 \quad$ p. 6

The binary encoding of the metafile is a logical data structure consisting of a sequential collection of bits.

B2: Clause 4.3

$\# 3 / 2$ p. 6

Metafile elements are constrained to start on word boundaries within the binary data structure (this alignment may necessitate padding an element with bits to a word boundary if the parameter data of the element does not fill to such a boundary).

B3: Clause 4.4

$\# 1 / 2 \quad$ p. 7

Metafile elements are represented in the Binary Encoding in one of two forms--short-form commands and long-form commands.

B4: Clause 4.4

$\# 2 / 1 \quad$ p. 7

The short-form command always contains a complete element.

B5: Clause 4.4

\#3/1 p.7

The short-form command only accommodates parameter lists up to 30 octets in length.

B6: Clause 4.4

\#3/1 p.7

The long-form command accommodates lengths up to 32767 octets per data partition.

B7: Clause 4.4

\#7/1-2 p. 8

The first word of a long-form command is identical in structure to the first word of a short-form command. The presence of the value 11111 binary (decimal 31) in the parameter list length field indicates that the command is a long-form command. 
B8: Clause 4.4

$\# 7 / 3-5 \quad$ p. 8

The Command Header for the long-form command consists of two words. The second word contains the actual parameter list length. The two header words are then followed by the parameter list.

B9: Clause 4.4

$\# 8 / 1-4 \quad$ p. 8

The long-form command allows the parameter list to be partitioned. Bit 15 of the second word indicates whether the given data complete the element or more data follow. For subsequent data partitions of the element, the first word of the long-form Command Header is omitted; only the second word, containing the parameter list length, is given.

B10: Clause 4.4

$\# 8 / 5 \quad$ p. 8

The parameter list length for each partition specifies the length of that partition, not the length of the complete element.

B11: Clause 4.4

$\# 8 / 56 \quad$ p. 8

The final partition of an element is indicated by bit 15 of the parameter list length word being zero.

B12: Clause 4.4

$\# 10 / 7 \quad$ p. 8

Unless otherwise stated, the ordex of parameters is as listed in clause 5 of part 1.

B13: Clause 4.4

$\# 1.1 / 1-2 \quad$ p. 8

Every command is constrained to begin on a word boundary. This necessitates padding the command with a single null octet at the end of the command if the command contains an odd number of octets of parameter data.

B1.4: Clause 4.4

$\# 11 / 3 \quad$ p. 9

In elements with parameters whose precisions are shorter than one octet (i.e., those containing a 'local colour precision' parameter) it is necessary to pad the last data-containing octet with null bits if the data do not fill the octet. 
B15: Clause 4.4

$\# 11 / 4 \quad$ p. 9

In all cases, the parameter list length is the count of octets actually containing parameter data--it does not include the padding octet if one is present. It is only at the end of a command that padding is performed, with the single exception of the CELL ARRAY element.

B16: Clause 4.4

$\# 14 / 1 \quad$ p. 9

The short form command header with element class 15, element id 127, and parameter list length 0 is reserved for extension of the number of available element classes in future revisions [of the standard].

[NOTE: This particular element should not be encountered in version 1 metafiles.]

\section{B17: Clause 5}

$\# 1 / 1-2 \quad$ p. 10

The Binary Encoding of the CGM uses five primitive data forms to represent the various abstract data types used to describe parameters in ISO $8632 / 1$. [These are signed Integer (SI), Unsigned Integer (UI), Character (C), Fixed Point Real (FX), and Floating point Real (FP).]

\section{B18: Clause 5}

$$
\text { \#5/2 p. } 10
$$

In general, parameters may align on odd or even octet boundaries, because they may be preceded by an odd or even number of octets of other parameter data.

\section{B19: Clause 5}

\#5/3-4 p. 10

Elements containing the local colour precision parameter may have parameters shorter than one octet. It is possible in such cases that the parameters will not align on octet boundaries.

B20: Clause 5.1
$\# 1 / 1$
p. 10

signed integers are represented in "two's complement format." 
B21: Clause 5.1
$\# 1 / 2$
p. 10

Four precisions may be specified for signed integers: 8-bit, 16bit, 24-bit, and 32-bit. Integer coordinate data encoded with this primitive data form do not use the 8-bit precision.

B22: Clause 5.2

$\# 1 / 1 \quad$ p. 11

Four precisions may be specified for unsigned integers: 8-bit, 16-bit, 24-bit, and 32-bit.

\section{B23: Clause 5.3}

$\# 1 / 1 \quad$ p. 12

Each character is stored in an octet [with the $i$-th character occupying the most significant octet of a word].

B24: Clause 5.4

$\# 1 / 1 \quad$ p. 12

Fixed point real values are stored as two integers; the first represents the "whole part" and has the same form as a signed Integer; the second represents the "fractional part" and has the same form as an Unsigned Integer.

B25: Clause 5.4

$\# 1 / 2 \quad$ p. 12

Two precisions may be specified for Fixed point Reals: $32-b i t$ and 64-bit.

B26: Clause 5.4 .3

$\# 1 / 1 \quad$ p. 13

The values of the represented [fixed point] real numbers are given by [specific equations in this clause of the standard].

B27: Clause 5.5

$\# 1 / 1 \quad$ p. 13

Floating point Real values are represented in the floating point format of ANSI/IEEE 754 . 
B28: Clause 5.5

$\# 2 / 2 \quad$ p. 13

Two precisions may be specified for Floating Point Reals: 32- bit or 64-bit.

B29: Clause 6

Note 3 p. 16

Abstract parameter type Enumeration, E, is encoded identically to abstract type Index, IX, at 16-bit precision.

B30: Clause 6

Note 6 p.16-17

A string is encoded as a count (unsigned integer) followed by characters. The encoding of the count is similar to the encoding of length information for metafile commands themselves. If the first octet is in the range $0 . .254$, then it represents the character count for the complete string. If the first octet is 255, then the next 16 bits contain the character count and a continuation flag. The first bit is used as a continuation flag (allowing strings longer than 32767 characters) and the next 15 bits represent the count, 0..32767, for the partial string. If the first bit is 0 , then this partial string completes the string parameter. If 1 , then this partial string will be followed by another.

[NOTE: This implies that a null string is encoded as a single octet with value 0.$]$

B31: Clause 6

Note $10 \quad$ p. 17

Fixed point reals apply to VDC and to $R$ parameters for the following elements: line width, edge width, character spacing, character expansion factor, marker size, vertical continuous text alignment, horizontal continuous text alignment.

[NOTE: This implies that the metric scale factor parameter of the SCALING MODE element may be represented only as a Floating Point Real.]

B32: Clause 6

Note 11 p.17

For PACKED [CELL ARRAY] mode, each row of the cell array is represented by an array of colour values without compression. Each row starts on a word boundary. 
B33: Clause 6

Note 11 p. 17

For RUN LENGTH encoding, the data for each row [of the cell array] begins on a word boundary and consists of run-lengthlists for runs of constant colour value.

B34: Clause 7.2

Code 0 p. 20

A NO-OP has 1 parameter, P1, [which consists of] an arbitrary sequence of $n$ octets, $n=0,1,2, \ldots$

[NOTE: This implies that the minimum NO-OP element consists of two octets each with value 0.]

B35: Clause 7.3

Code 12 p. 22

METAFILE DEFAULTS REPIACEMENT has 1 parameter that itself contains metafile elements. The structure and format is identical to appropriate metafile element(s).

[NOTE: This implies that all word alignment rules for metafile elements also apply to these elements when they are part of the parameter to METAFILE DEFAULTS REPLACEMENT.]

B36: Clause 7.4

Code 1 p. 24

[The second] SCALING MODE parameter is a (real) metric scaling factor which is ignored if [the first parameter] is 0 .

[NOTE: In Table 5, the real factor is always expressed as floating point (FP); fixed point is not allowed for this field or else the entry would have read $R$ not FP.]

B37: Clause 7.6

Code 9 p. 30

[In the eighth parameter of CELL ARRAY when cell representation mode is 'run length'] each list item consists of a cell count (integer) followed by a colour value. 
B38: Clause 7.7

Code 32 p. 36

[NOTE: NB: Unlike CELL ARRAY, pattern definitions in PATTERN TABLE have no "cell representation mode"; that is, they cannot be run-length encoded.]

B39: Clause 9

$\# 2 / 1 \quad$ p. 41

[A metafile conforms to this encoding if] each metafile element is coded in the manner described.

B40: Clause 9

\#3/1 p.41

[A metafile conforms to this encoding if] private metafile elements are all coded using the GDP or ESCAPE metafile elements as appropriate. Opcodes reserved for future standardization are not used to code private (non-standard) metafile elements.

[NOTE: This implies that a CGM is not conforming if it contains any opcodes (class/id pairs) not standardized in Iso 8632. ]

B41: Clause 9

$\# 4 / 1 \quad$ p. 41

[A metafile conforms to this encoding if] private values of index parameters are all coded using negative numbers.

B42: Clause 9

\#5/1 P. 41

[A metafile conforms to this encoding if] values specified as being "reserved for registration or future standardization" are not used unless their meaning has been registered.

[NOTE: Because no new elements have been registered or standardized, no such values should be encountered as yet.]

B43: Clause 9

$\# 7 / 1 \quad$ p. 41

A conforming metafile may include, within the string parameters of TEXT, RESTRICTED TEXT, and APPEND TEXT elements, as well as string parameters within the data records of GDP elements, the Iso 2022 controls for designating and invoking G- sets. This is an alternative way, in addition to CHARACTER SET INDEX, by which character sets for displaying text strings may be selected. 
B44: Annex C

a11 p.48-50

These are the codes used in the METAFILE ELEMENTS LIST element:
$0 / 1$ through $0 / 5$
$1 / 1$ through $1 / 15$
$2 / 1$ through $2 / 7$
$3 / 1$ through $3 / 6$
$4 / 1$ through $4 / 19$
$5 / 1$ through $5 / 35$
$6 / 1$
$7 / 1$ and $7 / 2$.

[NOTE: Note the omission of $0 / 0$ (NOOP). This requirement is stated in Annex $c$, but it merely summarizes the contents of the Clause 7 tables, so it should be upheld as a valid requirement.] 



\section{REQUIREMENTS EXTRACTED FROM MIL-D-28003}

\section{A. Explanation of Contents}

The Military Specification, Digital Representation for Communication of Illustration Data: CGM Application Profile, MIL-D-28003 (20 December 1988), was systematically read and all requirements relating to the definition of a CALS conforming basic metafile were extracted. Each separate statement of requirements is assigned a number and quoted. The requirement numbers are assigned sequentially from one and all start with the letter "M," indicating that these requirements come from MIL-D28003, the Milspec CGM Application Profile.

A citation for each statement (shown in boldface) is given according to the following scheme: The clause number is provided on the first line; on the second line, for most citations, the paragraph number (p) and sentence number(s) are specified as "\#p/s", followed by the page number on which the text occurs. For a few citations, the only difference is that the second line starts with a Table number, rather than a "\#". This means that the information is contained in the specified table on the specified page.

Any commentary on the requirements statement is shown as a note enclosed within brackets ([NOTE: ...]).

\section{B. Organization of Requirements}

The requirements are grouped into eight main categories. Within each category, the requirements are generally stated in order of their clause number. The only exception to this rule is when a requirement is stated in several places in the standard. These requirement statements are grouped together.

The eight categories of requirements statements are:

General CALS

Physical to Logical Mapping

Metafile structure and ordering

Parameter Decoding

Parameter Ranges

Size Constraints

GDP and ESCAPE Elements

Colour/Pattern Usage Rules.

In addition, MIL-D-28003 corrects several editorial errors found in ANS X3.122 adopted by FIPS PUB 128. These corrections are placed in their appropriate category and marked with the phrase (CORRECTION) preceding the Clause number on the first line of the citation. 


\section{General CALS}

M1: Clause 3.1

$1 / 5 \quad$ p. 5

A conforming basic metafile shall contain no elements or parameters outside of the Basic set.

M2: Clause 3.1 .3

$1 / 1 \quad$ p. 7

A conforming basic metafile shall not contain scalar values of parameter data outside the ranges specified by this specification.

M3: Clause 3.1 .4

$1 / 1 \quad$ p. 7

A conforming basic metafile shall use only the CGM Binary Encoding, as defined in FIPS PUB 128, part 3.

M4: Clause 3.2 .1
$1 / 1-2$
p. 7

The Basic set shall be defined by the limitations on Basic Values [noted in subclauses of this clause]. Where an element is not mentioned, it is implied that the Basic set shall include all values permitted in FIPS PUB 128.

M5: Clause 3.2 .3

$1 / 1 \quad$ p.11

The defaults of all elements in this Application Profile shall be as specified in Clause 6 of Part 1 of FIPS PUB 128.

M6: Clause 3.2 .3

$1 / 2 \quad$ p.11

Conforming basic metafiles are permitted to contain one or more METAFILE DEFAULTS REPLACEMENT elements to redefine any of these values.

\section{Physical to Logical Mapping}

M7: Clause 3.1 .5

$1 / 1 \quad$ p. 7

All basic metafiles conforming to this specification shall consist of 80 -octet records. 
M8: Clause 3.1 .5
$1 / 2$
p. 7

When [conforming basic meta]files are being transmitted on magnetic tape, the 80-octet logical records shall be blocked into 800-octet physical records.

\section{Metafile structure and ordering}

M9: (CORRECTION) Clause 3.1 .6

$5 / 1 \quad$ p. 7

Metafile Descriptor elements ... shall not be included in the METAFILE DEFAULTS REPLACEMENT [element].

M10: Clause 3.2 .6 .1

$1 / 6-7 \quad$ p. 13

If used, this ESCAPE element (Disable clearing of view surface: id -301; data record null) must appear in the Metafile Descriptor. This ESCAPE element shall be a basic capability of the CGM Application Profile under this specification.

M11: Clause 3.2 .6 .2

$1 / 6 \mathrm{ff} \quad$ p. 1.3

If used, this ESCAPE element (Device viewport: id -302; data record, a single string of text) must appear in the Picture Descriptor. This ESCAPE element shall be a basic capability of the CGM Application Profile under this specification.

M12: Clause 3.2 .6 .3

$1 / 4 \mathrm{ff} \quad$ p. 14

This ESCAPE element (Implicit colour table: id -303; data record, a single string of text) shall be allowed in the Metafile Descriptor. This ESCAPE element shall be a basic capability of the CGM Application Profile under this specification.

M13: Clause $3 \cdot 2 \cdot 7.1$

3/1-3 p.15

The METAFILE DEFAULTS REPLACEMENT element shall not be partitioned. Note that FIPS PUB 128 permits multiple occurrences of this element, so that partitioning is not required. Partitioning shall be permitted for all other elements. 


\section{Parameter Decoding}

M14: (CORRECTION) Clause 3.1 .6

$3 / 1 \quad$ p. 7

Part 3, p.17, item 11: The fraction numerator which is "pn $x$ " should be "pn $x$-1".

M15: (CORRECTION) Clause 3.1 .6

$4 / 1 \quad$ P. 7

Part 3, p.26, VDC REAL PRECISION: "3I" should be "E,2I".

M16: Clause $3 \cdot 2 \cdot 1.3$

$1 / 1-2 \quad$ p. 8

Note that the scale-factor parameter of SCALING MODE is always a floating-point number, even when REAL PRECISION has selected fixed point for other real numbers. It is not apparent in FIPS PUB 128 what the precision of this floating point parameter is when fixed point reals have been selected: its precision shall be $(0,9,23)$.

\section{Parameter Ranges}

M17: Clause 3.2 .1 .1

$1 / 1 \quad$ p. 8

The only constraint on delimiter elements shall be for NO-OP, and the basic values allowed shall be an arbitrary sequence of $n$ octets, $n=0 . .32767$.

M18: Clause 3.2 .1 .2

Table I p.8

The METAFILE DESCRIPTION element's string (a) shall include a substring briefly identifying company or product [and] (b) shall contain the substring, "MIL-D-28003/BASIC-1".

M19: Clause $3.2 \cdot 1.2$

Table I p.8

INTEGER PRECISION Basic Value is 16.

M20: Clause 3.2 .1 .2

Table I p.8

REAL PRECISION Basic Values are $(1,16,16)$ and $(0,9,23)$. 
M21: Clause 3.2 .1 .2

Table I p.8

INDEX PRECISION Basic Value is 16.

M22: Clause 3.2.1.2

Table I p.8

COLOUR PRECISION Basic Values are 8 and 16.

M23: Clause 3.2.1.2

Table I p.8

COLOUR INDEX PRECISION Basic Values are 8 and 16.

M24: Clause 3.2.1.2

Table I p.8

For FONT LIST, [up to] four simultaneous fonts are supported. The font names are selected from those in [clause] 3.2 .5 (q.v.).

M25: Clause 3.2 .5

$1 / 2-4 \quad$ p. 12

All of [the font names in Table VI] shall be considered basic capabilities of a basic metafile conforming to this specification. Any of these font [names] may appear in the FONT LIST element in a basic metafile that conforms to this specification. Font name shall be the concatenation of the string "HERSHEY:", to designate one of the Hershey fonts, and a "name string" to designate the particular typeface. 
M26: Clause 3.2 .5

Table VI p.12

The Basic font names are:

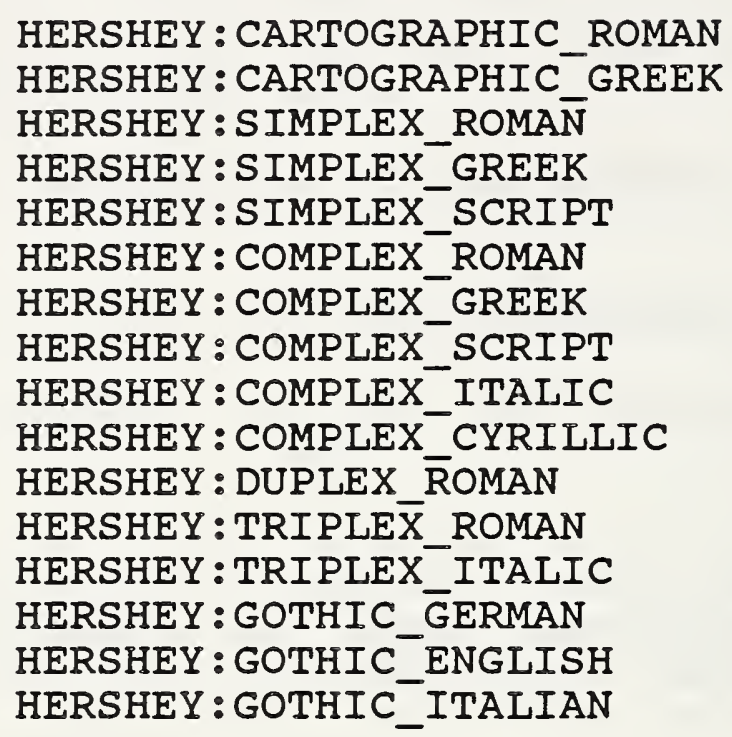

M27: Clause $3 \cdot 2 \cdot 1.2$

Table I p.8

CHARACTER SET LIST Basic Value is a two-element list: $\{(0,4 / 2),(1,4 / 1)\}$, which correspond to $\times 3.4$ (7-bit ASCII) and X3.134/2 (8-bit ASCII).

M28: Clause 3.2 .1 .2

Table I p.8

CHARACTER CODING ANNOUNCER Basic Values are 0 (Basic 7-bit) and 1 (Basic 8-bit).

M29: Clause 3.2 .1 .4

Table II p.9

VDC INTEGER PRECISION Basic Values are 16 and 32.

M30: Clause 3.2 .1 .4

Table II p.9

VDC REAL PRECISION Basic Values are $(1,16,16)$ (fixed) and $(0,9,23)$ (floating point). 
M31: Clause 3.2 .1 .4

Table II p.9

TRANSPARENCY Basic Value is 1 (on).

M32: Clause 3.2 .1 .6

Table III p.9-10

LINE BUNDLE INDEX Basic Values are 1-5.

M33: Clause 3.2.1.6

Table III p.9-10

LINE TYPE Basic Values are $1-5$ plus those defined in clause 3.2 .2 .1 .

M34: Clause 3.2 .2 .1

Table IV p.10

[Ten] additional line types specified in table IV shall apply. Their CGM parameter values are the consecutive integers - 11301 through -11310 .

M35: Clause 3.2 .1 .6

Table III p.9-10

MARKER BUNDLE INDEX Basic Values are 1-5.

M36: Clause 3.2 .1 .6

Table III p.9-10

MARKER TYPE Basic Values are 1-5.

M37: Clause 3.2 .1 .6

Table III p.9-10

TEXT BUNDLE INDEX Basic Values are 1-2.

M38: Clause 3.2 .1 .6

Table III p.9-10

TEXT. FONT INDEX Basic Values are $1-4$ and the character set selected shall be representable in the font selected.

M39: Clause 3.2.1.6

Table III p.9-10

CHARACTER SET INDEX Basic Values are 1-2 and the character set selected shall be representable in the font selected. 
M40: Clause 3.2.1.6

Table III p.9-10

ALTERNATE CHARACTER SET INDEX Basic Values are 1-2 and the character set selected shall be representable in the font selected.

M41: Clause 3.2.1.6

Table III p.9-10

FILL BUNDLE INDEX Basic Values are 1-5.

M42: Clause 3.2 .1 .6

Table III p.9-10

HATCH INDEX Basic Values are 1-6 plus the hatch styles (indexes) defined in clause 3.2.2.2.

M43: Clause 3.2.2.2

Table V p.11

[Eighteen] additional hatch styles specified in table $\mathrm{V}$ shall apply. Their CGM parameter values are the consecutive integers 11401 through -11407 and -11409 through -11418 .

M44: Clause 3.2 .1 .6

Table III p.9-10

EDGE BUNDLE INDEX Basic Values are 1-5.

M45: Clause 3.2.1.6

Table III p.9-10

EDGE TYPE Basic Values are 1-5.

M46: Clause 3.2 .1 .6

Table III p.9-10

PATTERN TABLE Basic Values are \{Starting Index: 1-8; $\mathrm{nx}$, ny: 1$16\}$.

M47: Clause 3.2 .1 .6

Table III p.9-10

COLOUR TABLE Basic Values are those with start index in the range $0-255$.

M48: Clause $3.2 \cdot 1.8$

$1 / 1 \quad$ p. 10

The "action required" flag of the MESSAGE element shall be restricted to the value "no action required". 


\section{Size Constraints}

M49: Clause 3.2 .8 .3

$3 / 1 \quad$ p. 18

The basic value for the number of colour values that can appear in a colour array or colour list parameter shall be: 1048576 for CELL ARRAY (one 1024x1024 image); 2048 for PATTERN TABLE (eight $16 \times 16$ patterns); 256 for COLOUR TABLE (entries 0-255).

M50: Clause 3.2 .8 .3

$5 / 1 \quad$ p. 18

The basic value for the number of points and VDC that can appear in parameters for metafile elements shall be 1024.

M51: Clause 3.2 .8 .3

$7 / 1 \quad$ p. 18

The basic value for the length of an individual string of characters shall be: 254 for all string parameters except data records; 32767 for data records.

\section{GDP and ESCAPE Elements}

M52: Clause 3.2 .1 .5

$1 / 1 \quad$ p. 9

Conforming basic metafiles shall not contain any Generalized Drawing Primitive (GDP) elements.

M53: Clause 3.2 .1 .7

$1 / 1 \quad$ p. 10

CGM application profiles conforming to this specification may contain only those ESCAPE elements that are defined in 3.2.6. 
M54: Clause $3 \cdot 2 \cdot 6.2$
$3 / 1 \mathrm{ff}$
p. 13-14

[For ESCAPE -302 the] escape data record [is] a single string of text containing the specification of the viewport. Parameters in the viewport shall be separated by at least one blank character and/or a single comma character. The decimal point of the real fraction shall be required. Leading zeros of the real fraction shall be optional. There are four parameters:

P1: First corner $\mathrm{x}$-coordinate. Real fraction of the default device viewport, in the range $[0.0,1.0]$.

P2: First corner $\mathrm{y}$-coordinate. Real fraction of the default device viewport, in the range $[0.0,1.0]$.

P4: Second corner $x$-coordinate. Real fraction of the default device viewport, in the range $[0.0,1.0]$.

P4: Second corner $y$-coordinate. Real fraction of the default device viewport, in the range $[0.0,1.0]$.

M55: Clause $3 \cdot 2 \cdot 6 \cdot 3$

$1 / 5$ p.14-15

The single integer parameter of the [-303] ESCAPE shall [be in the range] $0-2$. The default value shall be 1 .

M56: Clause $3 \cdot 2 \cdot 6 \cdot 3$

9/1-2 p.15

The [single] integer [parameter of the -303 ESCAPE shall be] encoded as "clear text," [e.g.,] value 2 is encoded as the string comprised of (or containing) the ASCII character "2".

\section{Colour/Pattern Usage Rules}

M57: Clause 3.2 .1 .6

$5 / 1-3 \quad$ p. 10

For indexed colour selection, either all colour indexes used in the metafile shall have their representations defined by use of the COLOUR TABLE element, or none shall. A colour index is "used" if it occurs in an element selecting a colour value to be applied to a primitive (LINE COLOUR, CELL ARRAY, etc.). A colour index is also "used" if it is the default for a primitive attribute and the default applies to a displayed primitive. 
M58: Clause $3.2 \cdot 7.1$
$7 / 2$
p. 16

If a COLOUR TABLE element defining the representation of a given colour index appears in a picture, it shall appear before reference to that index by an attribute element or use of that index by a graphical primitive element (included in the latter shall be implicit use of default colour index attribute values by the first occurrence of an associated primitive).

M59: Clause 3.2.7.1
$7 / 3$
p. 16

Once a given colour representation is defined and used, it shall not be redefined.

M60: Clause 3.2 .7 .1
$9 / 2$
p.16

If a PATTERN TABLE element defining the representation of a given pattern index appears in a picture: (a) it shall appear before explicit reference to that index by any PATTERN INDEX element; or (b) in the case of the default PATTERN INDEX, it shall appear before any implicit reference caused by the first occurrence of an associated filled primitive.

M61: Clause 3.2 .7 .1

$9 / 3 \quad$ p.16

Once a given pattern representation is defined and used, it shall not be redefined. 
VI. CROSS REFERENCE TABLE BETWEEN REQUIREMENTS AND TESTING

\section{Requirement}

F1

F2

F3

F 4

F5

F6

F7

F8

F9

F10

F11

F12

F13

F14

F15

F16

F17

F18

F19

F20

F21

F22

F23

F24

\section{Section Where Requirement is Represented for Testing}

II, step 6A

II, Step $6 \mathrm{~A}$

II, Step 6A

II, Step $6 \mathrm{~A}$

II, Step 4

II, Step 4

II, step 5

II, step 5

Appendix A

Appendix A

Appendix A

Appendix A

Appendix A

Appendix A

Appendix A

Appendix A

Appendix A

Appendix A

Appendix A

Appendix A

Appendix A

II, Step 6B

II, Step 6B

Appendix C, COLOUR VALUE EXTENT 


\section{Requirement}

F25

F26

F27

F28

F29

F30

F31

F32

F33

F34

F35

F36

F37

F38

F39

F4 0

F4 1

F4 2

F43

F 44

F45

F4 6

F47

F 48
Section Where Requirement is Represented for Testing Appendix C, COLOUR VALUE EXTENT

Appendix C, colour index setting elements

II, Step 5

II, Step 5

II, Step 5

II, step 5

Appendix A

Appendix A

Appendix A

Appendix A

Appendix A

Appendix A

Appendix A

Appendix A

Appendix A

Appendix A

Appendix A

Appendix A

Appendix C, string parameters of text elements Appendix C, string parameters of text elements Appendix C, string parameters of text elements Appendix C, METAFILE ELEMENT LIST, \& II, Step 5 Appendix C, METAFILE ELEMENT LIST, \& II, Step 5 Appendix C, METAFILE ELEMENT LIST, \& II, Step 5 
Requirement

F49

F50

F51

F52

F53

F54

F55

F56

F57

F58

F59

F'60

F61

F62

F63

F64

F65

F66

F67

F68

F69

F70
Section Where Requirement is Represented for Testing

Appendix C, METAFILE ELEMENT LIST, and II, Steps $4 \mathrm{~B}$ and $4 \mathrm{C}$

Appendix C, CELL ARRAY, and II, steps 4B and 4C Appendix C, colour setting elements, CELL ARRAY, PATTERN TABLE, and II, Steps $4 \mathrm{~B}$ and $4 \mathrm{C}$

Appendix C, VDC EXTENT

Appendix C, CELL ARRAY, and II, steps $4 B$ and $4 C$ Appendix C, PATTERN TABLE, \& II, steps $4 \mathrm{~B}$ and $4 \mathrm{C}$ Appendix C, LINE TYPE, MARKER TYPE, HATCH STYLE, EDGE TYPE, FONT LIST, GDP, and ESCAPE

Appendix $C$, index parameters

Appendix C, METAFILE VERSION

Appendix C, POLYLINE, and II, step 5

Appendix C, DISJOINT POLYLINE, and II, step 5

Appendix $C$, text elements

Appendix C, CELL ARRAY

Appendix C, CELL ARRAY

Appendix C, GDP

Appendix C, CIRCLE

Appendix C, CIRCULAR ARC CENTRE

Appendix C, CIRCULAR ARC CENTRE

Appendix C, CIRCULAR ARC CENTRE CLOSE

Appendix C, CIRCULAR ARC CENTRE CLOSE

Appendix C, ELLIPSE

Appendix C, ELLIPTICAL ARC 
Requirement

F71

F72

F73

F74

F75

F76

F77

F78

F79

F80

F81

F82

F83

F84

F85

F86

F87

F88

F89

F90

F91

F92

F93

F9 4
Section Where Requirement is Represented for Testing

Appendix C, ELLIPTICAL ARC

Appendix C, ELLIPTICAL ARC CLOSE

Appendix C, ELLIPTICAL ARC CLOSE

Appendix C, LINE BUNDLE INDEX

Appendix C, LINE TYPE

Appendix C, LINE WIDTH

Appendix C, MARKER BUNDLE INDEX

Appendix C, MARKER TYPE

Appendix C, MARKER SIZE

Appendix C, TEXT BUNDLE INDEX

Appendix C, FONT INDEX

Appendix $C$, CHARACTER EXPANSION FACTOR

Appendix C, CHARACTER HEIGHT

Appendix C, CHARACTER ORIENTATION

Appendix C, CHARACTER SET INDEX

Appendix C, ALTERNATE CHARACTER SET INDEX

Appendix C, FILL BUNDLE INDEX

Appendix C, INTERIOR STYLE

Appendix C, HATCH INDEX

Appendix C, PATTERN INDEX

Appendix C, EDGE BUNDLE INDEX

Appendix C, EDGE TYPE

Appendix C, EDGE WIDTH

Appendix C, PATTERN TABLE INDEX 
Requirement

F95

F96

F97

F98
Section Where Requirement is Represented for Testing

Appendix C, PATTERN TABLE

Appendix C, PATTERN SIZE

Appendix C, COLOUR TABLE

Appendix C, ESCAPE 


\section{Requirement}

B1

B2

B3

B4

B5

B6

B7

B8

B9

B10

B11

B12

B13

B14

B15

B16

B17

B18

B19

B20

B21

B22

B23

B2 4

B25
Section Where Requirement is Represented for Testing

II, step 1

II, step 2

II, step 2

II, step 2

II, step 2

II, step 2

II, step 2

II, step 2

II, step 2

II, step 2

II, step 2

II, step $4 B$

II, Step $4 B$

II, step $4 B$

II, Step $4 \mathrm{~B}$

II, step 3

II, step $4 \mathrm{C}$

II, step 4B

II, step $4 B$

II, step $4 \mathrm{C}$

Appendix C, signed integers

Appendix C, unsigned integers

II, step $4 \mathrm{C}$

Appendix C, fixed point reals

Appendix C, fixed point reals 
Requirement

B26

B27

B28

B29

B30

B31

B32

B33

B34

B35

B36

B37

B38

B39

B4 0

B4 1

B4 2

B43

B4 4
Section Where Requirement is Represented for Testing

Appendix $C$, fixed point reals

Appendix $C$, floating point reals

Appendix C, floating point reals

Appendix $C$, enumeration type parameters

Appendix C, strings

Appendix $C$, fixed point reals

II, step 4C, for CELL ARRAY

II, step 4C, for CELL ARRAY

Appendix $C$, NOOP

Appendix C, METAFILE ELEMENT LIST, \& II, Step 4

Appendix C, SCALING MODE

II, Step 4C, for CELL ARRAY

II, step $4 C$

II, step 3

II, step 3

Appendix C, index parameters

II, step 4D

Appendix C, string parameters

Appendix C, METAFILE ELEMENT LIST 


\section{Requirement}

M1

M2

M3

M4

M5

M6

M7

M8

M9

M10

M11

M12

M13

M14

M15

M16

M17

M18

M19

M20

M2 1

M22

M2 3

M24

M25
Section Where Requirement is Represented for Testing

II, Steps 3 and $4 B$

II, Step 4D

II, Steps 1 and 2

II, step 4D

II, Step 5

II, Step 5

II, Step 1

II, Step 1

Appendix A, METAFILE DEFAULTS REPLACEMENT

Appendix A, ESCAPE

Appendix A, ESCAPE

Appendix A, ESCAPE

II, Steps 2 and 5

Appendix B, CELL ARRAY

Appendix B, VDC REAL PRECISION

Appendix B, SCALING MODE, and II, Step 4D

Appendix $C$, NOOP

Appendix C, METAFILE DESCRIPTION

Appendix C, INTEGER PRECISION

Appendix C, REAL PRECISION

Appendix C, INDEX PRECISION

Appendix $C$, COLOUR PRECISION

Appendix C, COLOUR INDEX PRECISION

Appendix C, FONT LIST

Appendix C, FONT LIST 
Requirement

M2 6

M27

M28

M29

M30

M31

M32

M33

M34

M35

M36

M37

M38

M39

M40

M4 1

M4 2

M4 3

M44

M45

M4 6

M47

M4 8

M49
Section Where Requirement is Represented for Testing

Appendix $c$, FONT LIST

Appendix C, CHARACTER SET IIST

Appendix c, CHARACTER CODING ANNOUNCER

Appendix C, VDC INTEGER PRECISION

Appendix C, VDC REAL PRECISION

Appendix C, TRANSPARENCY

Appendix C, LINE BUNDLE INDEX

Appendix C, LINE TYPE

Appendix $C$, LINE TYPE

Appendix C, MARKER BUNDLE INDEX

Appendix C, MARKER TYPE

Appendix C, TEXT BUNDLE INDEX

Appendix C, TEXT FONT INDEX

Appendix C, CHARACTER SET INDEX

Appendix C, ALTERNATE CHARACTER SET INDEX

Appendix C, FILL BUNDLE INDEX

Appendix C, HATCH INDEX

Appendix C, HATCH INDEX

Appendix C, EDGE BUNDLE INDEX

Appendix $c$, EDGE TYPE

Appendix C, PATTERN TABLE

Appendix C, COLOUR TABLE

Appendix C, MESSAGE

Appendix C, CELL ARRAY, PATTERN TABLE, and COLOUR TABLE 


\section{Requirement}

M50

M51

M52

M53

M54

M55

M56

M57

M58

M59

M60

M61
Section Where Requirement is Represented for Testing Appendix C, point lists

Appendix $C$, string parameters

Appendix C, GDP, and II, step 5

Appendix C, GDP, and II, step 5

Appendix C, ESCAPE

Appendix C, ESCAPE

Appendix C, ESCAPE

II, Steps $4 B$ and $4 C$

II, step 5

II, step 5

II, Step 5

II, Step 5 
APPENDIX A

CGM ELEMENT ORDERING REQUIREMENTS 


\section{.}




\section{Explanation of Contents}

The CGM Functional Description, ISO 8632-1:1987, contains requirements regarding the permitted order of elements in a CGM. These requirements can best be summarized by describing a finitestate machine.

In the following, the states of the finite-state machine representing a CGM conformance-checking parser are listed, elements which cause state transitions are indicated, and which elements are valid in which states are indicated.

Seven states are needed:

$\begin{array}{ll}\text { MFCL } & \text { Metafile closed. } \\ \text { MDOP } & \text { Metafile Descriptor open, but first picture not yet } \\ & \text { open, and not processing a METAFILE DEFAUIS } \\ & \text { REPLACEMENT element. } \\ \text { Metafile Descriptor open, but first picture not yet } \\ \text { open; however, are processing a METAFILE DEFAULTS } \\ \text { REPLACEMENT element. } \\ \text { Picture Descriptor open, but not in Picture Body yet. } \\ \text { PDOP } \\ \text { Picture Body open, but not processing a "not final" } \\ \text { TEXT or "not final" RESTRICTED TEXT element. } \\ \text { Processing a "not final" TEXT or "not final" } \\ \text { PICL } \\ \text { RESTRICTED TEXT element. } \\ \text { First or subsequent picture closed, but metafile not } \\ \text { yet closed. }\end{array}$

In the following, all CGM elements are listed along with the states in which it is valid for them to occur. Any elements causing state transitions are annotated as (***), and are in boldface. The binary encoding element class and id codes are also provided.

MIL-D-28003, the CALS CGM Application Profile, places a few additional constraints on GDPs and ESCAPE elements. These constraints are noted with the phrase (CALS) in the left margin and highlighted in boldface. 
0,0

NO-OPERATION

0,1

BEGIN METAFILE

(***) Causes transition from MFCL to MDOP.

0,2

END METAFILE

0,3 first BEGIN PICTURE

(***) Causes transition from MDOP to PDOP.

0,3 second or later BEGIN PICTURE

(***) Causes transition from PICL to PDOP.

0,4 BEGIN PICTURE BODY

(*t*) Causes transition from PDOP to PBOP.

0,5 END PICTURE

(***) Causes transition from PBOP to PICL.

1,1

METAFILE VERSION

1,2

METAFILE DESCRIPTION

1,3

VDC TYPE

1,4

INTEGER PRECISION

REAL PRECISION

INDEX PRECISION

COLOUR PRECISION

COLOUR INDEX PRECISION

MAXIMUM COLOUR INDEX
1,8

1,9
MDOP

MDOP

MDOP

MDOP MMDR PDOP PBOP TXOP PICL MFCL

PBOP

MDOP

MDOP

MDOP

MDOP

MDOP

MDOP

MDOP

MDOP
PICL

PDOP 
1,10 COLOUR VALUE EXTENT MDOP

1,11 METAFILE ELEMENT LIST MDOP

1,12 METAFILE DEFAULTS REPLACEMENT MDOP

$(* * *)$ Causes transition from MDOP to MMDR.

(***) completion of last element contained in the METAFILE DEFAULTS REPLACEMENT parameter list causes transition from MMDR to MDOP.

1,13 FONT LIST MDOP

1,14 CHARACTER SET LIST MDOP

1,15 CHARACTER CODING ANNOUNCER MDOP

2, 1 SCALING MODE MMDR PDOP

2, 2 COLOUR SELECTION MODE MMDR PDOP

2,3 LINE WIDTH SPECIFICATION MODE MMDR PDOP

2,4 MARKER SIZE SPECIFICATION MODE MMDR PDOP

2,5 EDGE WIDTH SPECIFICATION MODE MMDR PDOP

2, 6 VDC EXTENT MMDR PDOP

2, 7 BACKGROUND COLOUR MIMDR PDOP

3, 1 VDC INTEGER PRECISION MMDR

3, 2 VDC REAL PRECISION

3, 3 AUXILIARY COLOUR MMDR

PBOP TXOP

3, 4 TRANSPARENCY MMDR

PBOP TXOP

3,5 CLIP RECTANGLE

PBOP MMDR 
3,6

4,1

POLYLINE

PBOP

4,2

DISJOINT POLYLINE

PBOP

4,3

POLYMARKER

PBOP

4,4

"final" TEXT

PBOP

4,4

"not final" TEXT

PBOP TXOP

(***) Causes transition from PBOP to TXOP.

4,5

"final" RESTRICTED TEXT

4,5

"not final" RESTRICTED TEXT

PBOP

PBOP TXOP

(***) Causes transition from PBOP to TXOP.

4,6

"not final" APPEND TEXT

TXOP

4,6

"final" APPEND TEXT

TXOP

(***) Causes transition from TXOP to PBOP. 4,7 POLYGON

PBOP

$4,8 \quad$ POLYGON SET

PBOP

4,9

CELL ARRAY

PBOP 
4,10 GENERALIZED DRAWING PRIMITIVE

PBOP

(CALS) This element is not permitted in a basic conforming metafile.

4,11

RECTANGLE

PBOP

4,12

CIRCLE

PBOP

4,13

CIRCULAR ARC 3 POINT

PBOP

4,14

CIRCULAR ARC 3 POINT CLOSE

PBOP

4,15

CIRCULAR ARC CENTRE

PBOP

4,16

CIRCULAR ARC CENTER CLOSE

PBOP

4,17

ELLIPSE

PBOP

4,18

ELLIPTICAL ARC

PBOP

4,19

ELLIPTICAL ARC CLOSE

PBOP

5,1

LINE BUNDLE INDEX

MMDR

5,2

LINE TYPE

MMDR

5,3

LINE WIDTH

MMDR

5,4

LINE COLOUR

MMDR

5,5

MARKER BUNDLE INDEX

MMDR PBOP 
5,6

5,7

5,8

5,9

5,10

5,11

5,12

5,13

5,14

5,15

5,16

5,17

5,18

5,19

5,20

5,21
MARKER TYPE

MARKER SIZE

MARKER COLOUR

TEXT BUNDLE INDEX

TEXT FONT INDEX

TEXT PRECISION

CHARACTER EXPANSION FACTOR

CHARACTER SPACING

TEXT COLOUR

CHARACTER HEIGHT

CHARACTER ORIENTATION

TEXT PATH

TEXT ALIGNMENT

CHARACTER SET INDEX

ALTERNATE CHARACTER SET INDEX

FILL BUNDLE INDEX
MMDR

PBOP

MMDR

PBOP

MMDR

PBOP

MMDR

PBOP TXOP

MMDR

PBOP TXOP

MMDR

PBOP TXOP

MMDR

PBOP TXOP

MMDR

PBOP TXOP

MMDR

PBOP TXOP

MMDR

PBOP TXOP

MMDR

PBOP

MMDR

PBOP

MMDR

PBOP

MMDR

PBOP TXOP

MMDR

PBOP TXOP

MMDR

PBOP 
$5,22 \quad$ INTERIOR STYLE

$5,23 \quad$ FILI COLOUR

5,24

HATCH INDEX

5,25 PATTERN INDEX

5,26 EDGE BUNDLE INDEX

$5,27 \quad$ EDGE TYPE

$5,28 \quad$ EDGE WIDTH

5,29

EDGE COLOUR

5,30

EDGE VISIBILITY

5,31

FILI REFERENCE POINT

5,32

PATTERN TABLE

5,33

PATTERN SIZE

5,34

COLOUR TABLE

5,35

ASPECT SOURCE FLAGS
PBOP

MMOP

PBOP

PBOP

PBOP MMDR

PBOP

MMDR

PBOP

MMDR

PBOP

PBOP

PBOP

MMDR

PBOP

MMDR

PBOP

PBOP

MMDR

PBOP

MMDR

PBOP 
6,1

(CAIS) Only the following three ESCAPEs are permitted in a basic conforming metafile and only in the specified states.

-301 Disable viewsurface clear

-302 Device viewport

-303 Implicit colour table

MDOP

PDOP

7, 1 MESSAGE

MDOP MMDR PDOP

PBOP PICL

7,2

APPLICATION DATA

MDOP MMDR PDOP

PBOP PICL 


\section{APPENDIX B}

CGM ELEMENT LENGTH REQUIREMENTS

\section{FROM}

ISO $8632-3: 1987$ 

The CGM Binary Encoding, ISO 8632-3:1987, Clause 7, contains tables showing, for each CGM element, the lengths of their parameter lists. Length is expressed as the number of octets occupied by the entire parameter list, but excluding the command header and any padding needed to provide word alignment.

The length of each parameter list is expressed as a function of a number of variables--among them are the various types and precisions allowed for each parameter. For an arbitrary combination of types and precisions, the table is rather complex to read and interpret. However, MIL-D-28003, the CGM Application profile for CALS, greatly restricts the number of types and precisions that are permitted to occur in a basic conforming metafile. Consequently, it is feasible to develop a parameter length table with many elements having a fixed length and others limited to only a few variations.

Variability in length is due to five major causes:

(1) VDC Type: If integer (I), all VDCs are 2 octets in length; if real $(R), 4$ octets in length.

(2) Colour Selection Mode and Precision: If indexed, colours are 1 or 2 octets in length; if direct, they are 3 or 6 octets, depending upon the value of colour index (CX8/CX16) and colour precision (CD8/CD16).

(3) String Length: Strings are either $n+1$ or $n+3$ octets long (depending on whether they are coded using the short form or the long form); because all strings in CALS (except the APPLICATION DATA data record) must contain fewer than 255 octets, for the purposes of this table it is assumed that all strings are coded with the short form.

(4) Number of Items in List: Several of the geometric primitive elements and some of the other elements (e.g., FONT LIST and ASPECT SOURCE FLAGS) consist of a list of elements, whose count is not directly available. The length is clearly a function of the number of items in the list.

(5) Local Colour precision: The numerous values allowed for local colour precision in the CELL ARRAY and PATTERN TABLE elements make for great variation in the length of the parameter list for these elcments.

In the following, all CGM elements are listed along with their lengths in octets. Where the length is a function of other variables, an expression giving the length in terms of these other variables is shown and a code is appended to shown the source of variability. In the table that follows, n is consistently used to represent the number of characters (octets) in a string and $\underline{m}$ to represent the number of items in a list; e.g., for the number of points in a "poly" geometric primitive. The binary encoding element class and id codes are also provided in the table. 


\begin{tabular}{|c|c|c|}
\hline 0,0 & NO-OPERATION & $\mathrm{m}$ \\
\hline 0,1 & BEGIN METAFILE & $n+1$ \\
\hline 0,2 & END METAFILE & 0 \\
\hline 0,3 & BEGIN PICTURE & $n+1$ \\
\hline 0,4 & BEGIN PICTURE BODY & 0 \\
\hline 0,5 & END PICTURE & 0 \\
\hline 1,1 & METAFILE VERSION & 2 \\
\hline 1,2 & METAFILE DESCRIPTION & $n+1$ \\
\hline 1,3 & VDC TYPE & 2 \\
\hline 1,4 & INTEGER PRECISION & 2 \\
\hline 1,5 & REAL PRECISION & 6 \\
\hline 1,6 & INDEX PRECISION & 2 \\
\hline 1,7 & COLOUR PRECISION & 2 \\
\hline 1,8 & COLOUR INDEX PRECISION & 2 \\
\hline 1,9 & MAXIMUM COLOUR INDEX & $\begin{array}{ll}1 & (\mathrm{CX} 8) \\
2 & (\mathrm{CX16})\end{array}$ \\
\hline 1,10 & COLOUR VALUE EXTENT & $\begin{aligned} 6 & (\mathrm{CD} 8) \\
12 & (\mathrm{CD} 16)\end{aligned}$ \\
\hline 1,11 & METAFILE ELEMENT LIST & $4 m+2$ \\
\hline 1,12 & METAFILE DEFAULTS REPLACEMENT & variable \\
\hline 1,13 & FONT LIST & $m *(n+1)$ \\
\hline 1,14 & CHARACTER SET LIST & $m *(n+3)$ \\
\hline 1,15 & CHARACTER CODING ANNOUNCER & 2 \\
\hline
\end{tabular}




\begin{tabular}{|c|c|c|c|}
\hline 2, & 1 & SCALING MODE & 6 \\
\hline 2, & 2 & COLOUR SELECTION MODE & 2 \\
\hline 2, & 3 & LIIVE WIDTH SPECIFICATION MODE & 2 \\
\hline 2, & 4 & MARKER SIZE SPECIFICATION MODE & 2 \\
\hline 2, & 5 & EDGE WIDTH SPECIFICATION MODE & 2 \\
\hline 2, & 6 & VDC EXTENT & $\begin{aligned} 8 & (I) \\
16 & (R)\end{aligned}$ \\
\hline 2, & 7 & BACKGROUND COLOUR & $\begin{array}{ll}3 & (\mathrm{CD} 8) \\
6 & (\mathrm{CD16})\end{array}$ \\
\hline 3, & 1 & VDC INTEGER PRECISION & 2 \\
\hline 3, & 2 & VDC REAL PRECISION & 6 \\
\hline 3 , & 3 & AUXILIARY COLOUR & $\begin{array}{ll}3 & (\mathrm{CD} 8) \\
6 & (\mathrm{CD16})\end{array}$ \\
\hline 3, & 4 & TRANSPARENCY & 2 \\
\hline 3, & 5 & CLIP RECTANGLE & $\begin{aligned} 8 & (I) \\
16 & (R)\end{aligned}$ \\
\hline 3, & 6 & CIIP INDICATOR & 2 \\
\hline 4, & 1 & POLYLINE & $\begin{array}{ll}4 m & (I) \\
8 m & (R)\end{array}$ \\
\hline 4, & 2 & DISJOINT POLYLINE & $\begin{array}{ll}4 m & (I) \\
8 m & (R)\end{array}$ \\
\hline 4 , & 3 & POLYMARKER & $\begin{array}{ll}4 m & (I) \\
8 m & (R)\end{array}$ \\
\hline 4, & 4 & TEXT & $\begin{array}{r}7+n(I) \\
11+n(R)\end{array}$ \\
\hline 4, & 5 & RESTRICTED TEXT & $\begin{array}{l}11+n(I) \\
19+n(R)\end{array}$ \\
\hline 4 & 6 & APPEND TEXT & $3+n$ \\
\hline
\end{tabular}



4,7
POLYGON
$4 \mathrm{~m}$ (I)
$8 m(R)$
4,8
POLYGON SET
$6 \mathrm{~m} \mathrm{(I)}$
$10 \mathrm{~m}(\mathrm{R})$
4,9
CELL ARRAY
$20+$ colour values (I)
$32+$ colour values (R)
4,10
GENERALIZED DRAWING PRIMITIVE
$5+4 m+n(I)$
$5+8 m+n(R)$
4,11
RECTANGLE
8 (I)
$16(\mathrm{R})$
4,12
CIRCLE
6 (I)
10 (R)
4,13
CIRCULAR ARC 3 POINT
12 (I)
24 (R)
4,14
CIRCULAR ARC 3 POINT CLOSE
14 (I)
$26(\mathrm{R})$
4,15
CIRCULAR ARC CENTRE
14 (I)
28 (R)
4,16
CIRCULAR ARC CENTER CLOSE
16 (I)
30 (R)
4,17
ELLIPSE
12 (I)
24 (R)
4,18
ELLIPTICAL ARC
20 (I)
32 (R)
4,19
ELLIPTICAL ARC CLOSE
22 (I)
34 (R)
5,1
LINE BUNDLE INDEX
2
5,2
LINE TYPE
2
5,3
LINE WIDTH
$\begin{array}{ll}2 & \text { (I) } \\ 4 & (\mathrm{R})\end{array}$ 


\begin{tabular}{|c|c|c|}
\hline 5,4 & LINE COLOUR & $\begin{array}{ll}1 & (\mathrm{CX} 8) \\
2 & (\mathrm{CX16}) \\
3 & (\mathrm{CD} 8) \\
6 & (\mathrm{CD} 16)\end{array}$ \\
\hline 5,5 & MARKER BUNDLE INDEX & 2 \\
\hline 5,6 & MARKER TYPE & 2 \\
\hline 5,7 & MARKER SIZE & $\begin{array}{ll}2 & (\mathrm{I}) \\
4 & (\mathrm{R})\end{array}$ \\
\hline 5,8 & MARKER COLOUR & $\begin{array}{ll}1 & (\mathrm{CX8}) \\
2 & (\mathrm{CX16}) \\
3 & (\mathrm{CD} 8) \\
6 & (\mathrm{CD16})\end{array}$ \\
\hline 5,9 & TEXT BUNDLE INDEX & 2 \\
\hline 5,10 & TEXT FONT INDEX & 2 \\
\hline 5,11 & TEXT PRECISION & 2 \\
\hline 5,12 & CHARACTER EXPANSION FACTOR & 4 \\
\hline 5,13 & CHARACTER SPACING & 4 \\
\hline 5,14 & TEXT COLOUR & $\begin{array}{ll}1 & (\mathrm{CX8}) \\
2 & (\mathrm{CX16}) \\
3 & (\mathrm{CD} 8) \\
6 & (\mathrm{CD} 16)\end{array}$ \\
\hline 5,15 & CHARACTER HEIGHT & $\begin{array}{ll}2 & (\mathrm{I}) \\
4 & (\mathrm{R})\end{array}$ \\
\hline 5,16 & CHARACTER ORIENTATION & $\begin{array}{l}8(I) \\
16(R)\end{array}$ \\
\hline 5,17 & TEXT PATH & 2 \\
\hline 5,18 & TEXT ALIGNMENT & 12 \\
\hline 5,19 & CHARACTER SET INDEX & 2 \\
\hline 5,20 & ALTERNATE CHARACTER SET INDEX & 2 \\
\hline 21 & FILL BUNDLE INDEX & 2 \\
\hline
\end{tabular}


5,22

5,23

5,24

5,25

5,26

5,27

5,28

5,29

5,30

5,31

5,32

5,33

5,34

5,35

6,1

7,1

7,2
INTERIOR STYLE

FILL COLOUR

HATCH INDEX

PATTERN INDEX

EDGE BUNDLE INDEX

EDGE TYPE

EDGE WIDTH

EDGE COLOUR

EDGE VISIBILITY

FILL REFERENCE POINT

PATTERN TABLE

PATTERN SIZE

COLOUR TABLE

ASPECT SOURCE FLAGS

ESCAPE

MESSAGE

APPLICATION DATA
2

1 (CX8)

2 ( $\mathrm{CX16)}$

3 (CD8)

6 (CD16)

2

2

2

2

$\begin{array}{ll}2 & (\mathrm{I}) \\ 4 & (\mathrm{R})\end{array}$

1 (CX8)

2 (CX16)

3 (CD8)

6 (CD16)

2

4 (I)

8 (R)

$8+$ colour values

8 (I)

$16(R)$

$1+3 m(C X 8, C D 8)$

$2+3 m(C \times 16, C D 8)$

$1+6 \mathrm{~m}$ (CX8,CD16)

$2+6 m(C X 16, C D 16)$

$4 m$

$3+n$

$3+n$

$3+n$ (short DR)

$5+n$ (long DR) 


\section{APPENDIX C}

ALLOWABLE PARAMETER RANGES

FOR

BINARY ENCODED CGM ELEMENTS 



\section{Element}

Parameter

NOOP

(CALS)

BEGIN METAFILE

(CALS)

END METAFILE

BEGIN PICTURE

(CALS)

BEGIN PICTURE

BODY

END PICTURE $\mathrm{n} / \mathrm{a}$

METAFILE

VERSION

METAFILE

DESCRIPTION

(CALS)

VDC TYPE

INTEGER

PRECISION

(CALS)

REAL

PRECISION

(CALS) length of string (PI)

contents of string (P1)

number of null-valued octets

length of string

$\mathrm{n} / \mathrm{a}$

length of string (PI)

contents of string (P1)

length of string

$\mathrm{n} / \mathrm{a}$

version number (P1)

length of string (P1)

contents of string (PI)

length of string

contents of string

type (PI)

precision (PI)

format (PI)

field width 1 (P2)

field width 2 (P3)
Parameter Range

$>=0$

$<=32767$

$>=0$

iset of legal

character codes

$<=254$

$>=0$

iset of legal

character codes\}

$<=254$

$=1$

$>=0$

iset of legal

character codes\}

$<=254$

contains

"MIL-D-28003/BASIC-1"

and a product name

$\{0,1\}$

$\{8,16,24,32\}$

$\{16\}$

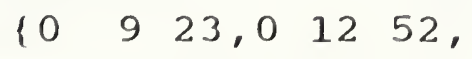

$\begin{array}{lllll}1 & 16 & 16,1 & 32 & 32\}\end{array}$

$\left\{\begin{array}{lllll}0 & 9 & 23,1 & 16 & 16\end{array}\right\}$ 
Element

INDEX

PRECISION

(CALS)

COLOUR

PRECISION

(CAIS)

COLOUR INDEX

PRECISION

(CALS)

MAXIMUM

COLOUR INDEX

(CAIS)

COLOUR VALUE

EXTENT

METAFILE

ELEMENT LIST

METAFILE

DEFAULTS

REPLACEMENT

FONT LIST

(CALS)

CHARACTER

SET LIST

(CAIS)
Parameter

precision (P1)

precision (PI)

$\{8,16,24,32\}$

$\{8,16\}$

precision (P1)

$\{8,16,24,32\}$

$\{8,16\}$

index value (P1)

$>0$

$<256$

minRed mingreen minBlue

maxRed maxGreen maxBlue

$>=0$

$>$ corresponding

minimum values

number of elements (P1)

for each (class/id)

$>0$

One of the valid

class/id pairs or

$-1 / 0$ or $-1,1$

$\mathrm{n} / \mathrm{a}$; however, within the span of the MDR element only completely specified Picture Descriptor, Control, Attribute, and Escape elements are allowed.

for each string in the list:

length of string (PI)

contents of string

number of font names in list

length of each string

$>=0$

\{ set of legal

character codes

$<=4$

$<=254$

for each item in list:

type

length of string (P2)
$>=0$ AND $<=4$ or negative

$>0$

$\{(0,4 / 2),(1,4 / 1)\}$ 
Element

CHARACTER

CODING

ANNOUNCER

(CATS)

SCALING MODE

(CAIS)

COLOUR

SELECTION

MODE

LINE WIDTH

SPECIFICATION

MODE

MARKER SIZE

SPECIFICATION

MODE

EDGE WIDTH

SPECIFICATION

MODE

VDC EXTENT

BACKGROUND

COLOUR

VDC INTEGER

PRECISION

(CAIS)

VDC REAL

PRECISION

(CALS)

\section{Parameter}

coding technique

$\{0,1\}$

mode (P1)

metric scale factor (P2)

metric scale factor (P2)

mode (P1)

mode (P1)

$\{0,1\}$

mode (PI)

$\{0,1\}$

mode (P1)

$\{0,1\}$

$\mathrm{n} / \mathrm{a}$

Red Green Blue

each component $>=$ corresponding min. coloux extent AND $<=$ corresponding max. colour extent

precision (P1)

$\{16,24,32\}$

$\{16,32\}$

format (PI)

field width 1 (P2)

field width 2 (P3)

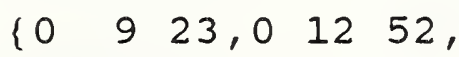

$\left.\begin{array}{lllll}1 & 16 & 16,1 & 32 & 32\end{array}\right\}$

$\left\{\begin{array}{lllll}0 & 9 & 23,1 & 16 & 16\end{array}\right\}$ 
Element

\section{AUXILIARY \\ COLOUR}

TRANSPARENCY

(CALS)

CLIP

RECTANGLE

CLIP

INDICATOR

POLYLINE

(CAIS)

DISJOINT

POLYLINE

(CALS)

POLYMARKER

(CALS)

TEXT

(CALS)

RESTRICTED

TEXT

(CALS) colour index

or

(P1)

Red Green Blue

indicator (P1)

$\mathrm{n} / \mathrm{a}$

indicator (P1)

$\mathrm{n} / \mathrm{a}$

number of points

$\mathrm{n} / \mathrm{a}$

number of points

$\mathrm{n} / \mathrm{a}$

number of points

flag

length of string (P1)

contents of string

length of string

flag

length of string (P1)

contents of string

length of string
$\{1\}$

$\{0,1\}$

Parameter Range

$<=$ maximum

colour index

each component >= corresponding min. colour extent AND

$<=$ corresponding max. colour extent

\section{$\{0,1\}$}$$
1\}
$$

[would like number of points $>=2$ ]

$<=1024$

[would like number of points to be even AND $>=2$ ]

$<=1024$

$<=1024$

$\{0,1\}$

$>=0$

(set of legal character codes\}

$<=254$

$\{0,1\}$

$>=0$

(set of legal

character codes\}

$<=254$ 
Element

APPEND TEXT flag

(CALS)

POLYGON

(CALS)

POLYGON SET

(CALS)

CELL ARRAY
Parameter

length of string (P1)

contents of string

length of string

$\mathrm{n} / \mathrm{a}$

number of points

for each pair of values in list: edge out flag

for each polygon in set

number of points

$P, Q, R(P 1, P 2, P 3)$

$\mathrm{nx}$ (P4)

ny (P5)

local colour precision (P6)

cell rep. mode ( $\mathrm{P} 7$ )

for each cell colour value: colour index

or

Red Green Blue

(CALS)

GDP

(CALS)

RECTANGLE
$\mathrm{nx}(\mathrm{P} 4)$

ny (P5)

identifier (P1)

number of points (P2)

length of data record string

No GDP is allowed.

$\mathrm{n} / \mathrm{a}$
Parameter Range

$$
\begin{aligned}
& \{0,1\} \\
& >=0 \\
& \{\text { set of legal } \\
& \text { character codes\} } \\
& <=254
\end{aligned}
$$

[would like number of points $>=3$ ]

$<=1024$

$\{0,1,2,3\}$

[would like number of points $>=3$ ]

$<=1024$

$\mathrm{RQ} \times \mathrm{RP} /=0$

$>=1$

$>=1$

$\{0,1,2,4$,

$8,16,24,32\}$

$\{0,1\}$

$>0$ AND $<=\max$.

colour index

each component $>=$ corresponding min. colour extent AND $<=$ corresponding max. colour extent

$<=1024$

$<=1024$

$<=\mathrm{m}$, where

$\mathrm{m}=$ highest

registered id.

[ $m=-1$ now]

$>=0$

$>=0$ 
Element

Parameter

CIRCLE

CIRCUIIAR ARC

CIRCUIAR ARC

3 POINT

CIRCULAR ARC

3 POINT CLOSE

CIRCULAR ARC CENTRE

CIRCULAR ARC CENTRE CLOSE

ELLIPSE
ELLIPTICAL
ARC

ELLIPTICAL ARC CLOSE

LINE BUNDLE INDEX

(CALS)

LINE TYPE

type (P1)

start vector (P2)

End vector (P3)

radius ( $\mathrm{P} 4$ )

start vector (P2)

End vector (P3)

radius ( $\mathrm{P} 4$ )

close type (P5)

Center (P1)

First CDP (P2)

second CDP (P3)

Center (PI)

First CDP (P2)

second CDP (P3)

start vector (P4)

End vector (P5)

Center (P1)

First CDP (P2)

second CDP (P3)

start vector (P4)

End vector (P5)

close type (P6)

index (P1)

LINE TYPE tYpe (P1)

\section{Parameter Range}

$$
>=0.0
$$

$\{0,1\}$

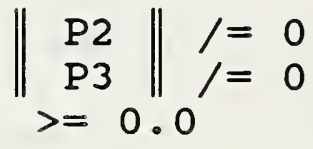

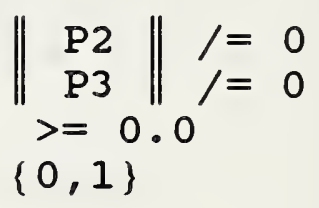

$\mathrm{P} 1 /=\mathrm{P} 2$

$\mathrm{P} 2 /=\mathrm{P} 3$

P3 $/=$ P1

P4
P5
$\{0,1\}$

$>0$

$\{1 . .5\}$

$<0$ OR $\{1 \ldots \mathrm{m}\}$

where $\mathrm{m}=$ highest

registered or

standardized line type. [ $m=5$ now].

\{1..5 and

$-11301 \ldots-11310\}$ 


\section{Element}

LINE WIDTH

LINE COLOUR

MARKER BUNDLE

INDEX

(CALS)

MARKER TYPE

(CAIS)

MARKER SIZE

MARKER COLOUR

\section{TEXT BUNDLE}

INDEX

(CALS)

TEXT FONT

INDEX

\section{Parameter}

VDC width or scale factor

colour index

or

Red Green Blue

index (P1)

type (P1)

VDC size or scale factor

colour index

Red Green Blue

index (P1)

index (P1)
Parameter Range

$>=0$ or $>=0.0$

depending on

VDC type and

specification mode

$<=$ maximum

colour index

each component >= corresponding min. colour extent AND $<=$ corresponding max. colour extent

$>0$

$\{1 \ldots 5\}$

$<0$ OR $\{1 \ldots \mathrm{m}\}$

where $\mathrm{m}=$ highest

registered or standard marker type. [m=5 now].

$\{1 . .5\}$

$>=0$ or $>=0.0$

depending on

VDC type and

specification mode

$<=$ maximum

colour index

each component >= corresponding min. colour extent AND

$<=$ corresponding

max. colour extent

$>0$

$\{1,2\}$

$>0$ [perhaps AND $<=$ number of fonts in font list] 
(CALS)

TEXT PRECISION

CHARACTER

EXPANSION

FACTOR

CHARACTER

SPACING

TEXT COLOUR

CHARACTER

HEIGHT

CHARACTER

ORIENTATION

TEXT PATH

TEXT

ALIGNMENT

CHARACTER

INDEX

(CALS)

ALTERNATE

CHARACTER

SET INDEX

(CALS)

FILL BUNDLE INDEX precision (P1)

expansion factor (P1)

$\mathrm{n} / \mathrm{a}$

colour index

or

Red Green Blue

VDC height (PI)

character up vector (P1)

character base vector (P2)

path (P1)

horizontal alignment (P1)

vertical alignment (P2)

index (P1)

index (P1)

index (P1)
Parameter Range

$$
\{1 \ldots 4\}
$$

$\{0,1,2\}$

$>=0.0$

$<=$ maximum colour index

each component >= corresponding min. colour extent AND $<=$ corresponding max. colour extent

$>=0$ or $>=0.0$

depending on VDC type

$\|$ P1
P2

$\{0,1,2,3\}$

$\{0,1,2,3,4\}$

$\{0,1,2,3,4,5,6\}$

$>0$ [perhaps AND SET

$<=$ number in character set list]

$\{1,2\}$

$>0$ [perhaps AND $<=$ number in character set 1 ist]

$(1,2)$

$>0$ 


\section{Element}

Parameter

\section{(CALS)}

INTERIOR

STYLE

FILL COLOUR

HATCH INDEX

(CALS)

PATTERN

INDEX

EDGE BUNDLE

INDEX

(CALS)

EDGE TYPE

(CALS)

EDGE WIDTH style (P1)

colour index

Red Green Blue

index (P1)

index (P1)

index (PI)

type (P1)

VDC width or scale factor
Parameter Range

$$
\begin{aligned}
& \{1 \ldots 5\} \\
& <=4 \\
& <=\underset{\quad \text { maximum index }}{\text { colour in }}
\end{aligned}
$$

each component >= corresponding min. colour extent AND $<=$ corresponding max. colour extent

$<0$ OR $\{1 \ldots \mathrm{m}\}$ where $\mathrm{m}=$ highest registered or standard hatch index. $[m=6$ now $]$.

$\{1 \ldots 6$ and

$-11401 . .-11407$,

$-11409 . .-11418\}$

$>0$

$>0$

$\{1 \ldots 5\}$

$<0$ OR $\{1 \ldots \mathrm{m}\}$ where $\mathrm{m}=$ highest registered or standardized edge type. [ $m=5$ now].

$\{1 . .5\}$

$>=0$ or $>=0.0$

depending on VDC type and specification mode 
Element

EDGE COLOUR

EDGE
VISIBILITY

FILL

REFERENCE

POINT

PATTERN

TABLE

(CAIS)

PATTERN SIZE

COLOUR TABLE
Parameter

colour index

or

Red Green Blue

visibility

$\mathrm{n} / \mathrm{a}$

index (P1)

$\mathrm{nx}$ (P2)

ny (P3)

local colour precision (P4)

for each cell colour value: colour index

or

Red Green Blue

index (P1)

$\mathrm{nx}$ (P2)

ny (P3)

pattern height vector (P1)

pattern width vector (P2)

starting colour index

for each colour to be loaded:

Red Green Blue
Parameter Range

$<=$ maximum colour index

each component >= corresponding min. colour extent AND $<=$ corresponding max. colour extent $\{0,1\}$

$$
\begin{aligned}
& >0 \\
& >=1 \\
& >=1 \\
& \{0,1,2,4 \text {, } \\
& 8,16,24,32\}
\end{aligned}
$$

each component >= corresponding min. colour extent AND $<=$ corresponding max. colour extent

$1<=$ and $<=8$

$1<=$ and $<=16$

$1<=$ and $<=16$

$\|$\begin{tabular}{l||l} 
P1 & $/=0$ \\
P2 & $/=0$
\end{tabular}

height $\mathrm{x}$ width $/=0$

$<=\operatorname{maximum}$ colour index

each component $>=$ corresponding min. colour extent AND $<=$ corresponding max. colour extent 
also

(CALS)

ASPECT

SOURCE FLAGS

ESCAPE

(CALS)

MESSAGE

(CALS)

APPLICATION DATA

(CALS) starting colour index

for each pair of values:

type

value

identifier (P1)

length of data record string

identifier content of data record is prescribed

action flag (P1)

len. of message string (P2)

action flag

length of data record string

length of data record string

$$
\begin{aligned}
& \text { total-number-of } \\
& \text { colours-loaded } \\
& \text { plus starting- } \\
& \text { colour-index - } 1 \\
& \text { should not }> \\
& \text { max. colour index } \\
& <256
\end{aligned}
$$

$$
\{0, \ldots 17\}
$$

$<=\mathrm{m}$, where $\mathrm{m}=$ highest registered id. $[m=-1$ now $]$ $>=0$

$\{-301 \ldots-303\}$

\section{$\underline{\text { KEY: }}$}

$\|\mathrm{Px}\|$ means the mathematical NORM of the vector specified in parameter $\mathrm{Px}$.

vectorl $\mathrm{x}$ vector 2 means the vector cross product

[... ] text in the square brackets contains additional information for the tester.

$i=$ means not equal to

$<=\quad$ means less than or equal to

$>=$ means greater than or equal to 



FINAL REPORT

CAIS FY89 SOW TASK 4.1 .3

INJECTION OF CALS REQUIREMENTS

IN EXTENDED CGM (CGEM) STANDARDS EFFORTS 

1. Foreword . . . . . . . . . . . . . . . . . 1

2. Summary and Conclusions . . . . . . . . . . . . 1

3. Recommendations .. . . . . . . . . . . . . 2

4. Activities in FY89 . . . . . . . . . . . . . . 3

4.1 Motivation for CGEM ................. 3

4.2 Historical overview . . . . . . . . . . . . 3

4.3 Review of Goals for FY89 .............. 5

4.4 Key Activities of 1989 . . . . . . . . . . . . . . 6

4.4.1 Addendum 1................. 6

4.4.1.1 Initial Status and Goals . . . . . 6

4.4.1.2 Houston X3H3 Meeting . . . . . . 6

4.4.1.3 DAD Ballot \& ANSI Letter Ballot. . . 7

4.4.1.4 X3H3 Milpitas Meeting . . . . . . 7

4.4.1.5 Between Milpitas and Waikoloa. . . . 8

4.4.1.6 Waikoloa MRG Meeting .. . . . . 8

4.4.1.7 Nashua X3H3 Meeting........ . 11

4.4.1.8 Status \& Remaining Work . . . . . . . 11

4.4.1.9 ANSI Processing of Addendum 1 . . . . 12

4.4.2 Addendum 3. . . . . . . . . . . . . . . . 12

4.4.2.1 Initial Status and Goals . . . . . . 12

4.4.2.2 Redondo Beach Text Meeting . . . . . 13

4.4.2.3 Munich Study Group Meeting . . . . . 14

4.4.2.4 Milpitas X3H3 Meeting: Liaisons and

4.4 .2 .5 Drafting $\dot{0} \cdot \dot{0} \cdot \dot{0}$. $\cdot \cdot \cdot \cdot 15$

4.4.2.6 Waikoloa Meeting . . . . . . . 16

4.4.2.7 Post Waikoloa \& ANSI Letter Ballot . 17

4.4.2.8 Nashua X3H3 Meeting... . . . . 17

4.4.2.9 Status \& Remaining Work . . . . . . 18

4.4.3 Addendum 2 . . . . . . . . . . . . . . . . . 19

4.4.4 Coordination between CGM Addenda and
Graphical Registration....... . . 20 


\section{TABLE OF CONTENTS (Continued)}

Attachment 1: US Vote and Comments on CGM Addendum 1 DAD Ballot . . . . . . . . . . . . . 21

Attachment 2: US Contribution to the Improved Graphical Text Model ................ 31

Attachment 3: Minutes of the Munich CGM Extension Meeting - 45 Attachment 4: Addendum 3 New Work Item Proposal Addendum 3 Requirements Document . . . . . 55

Attachment 5: Final Report of CGM Extension Study Period - 63 Attachment 6: Addendum 3 Working Draft . . . . . . . 73 Attachment 7: US Comments on Addendum 3 Working Draft . . 115 Attachment 8: Draft Addendum Text for CGM Addendum 1 • . 127 


\section{Foreword}

NIST/NCSL has in the past participated in the CGEM work through the CALS representative, Lofton Henderson, on the ANSI and ISO committees, and has continued this participation in FY89. In FY89, which ended on september 30, 1989, the work for this project had three principle aspects: the working meetings of the graphics and metafile experts of ANSI and ISO; inter-meeting work of preparing and coordinating position papers, baseline standards documents, and ballot responses; coordination and liaison with the Graphical Registration work.

This final report summarizes the progress that was made at the working meetings, the current status of CGM extensions work, projected timetables for completion, and recommendations for future work. This report also presents details of key domestic and international meetings for CGM extensions during FY89. Finally, key documents such as standards drafts, study reports, and US position papers which are a result of work under this contract are included as attachments.

\section{Summary and Conclusions}

Previous work has focussed on defining the CALS requirements for CGEM, getting those requirements endorsed by ANSI, and introducing those requirements into the Iso CGM addenda processing. The requirements definition and ANSI X3H3 endorsement were largely accomplished. Getting functionality into CGM Addendum 1 that meets some of the CALS needs has been largely accomplished. The major uncompleted work at the start of this fiscal year was getting ISO endorsement of the need for further extensions, the Addendum 3 project, and getting technical work underway on the project.

Specific goals for FY89 were:

1. Complete processing of CGM Addendum 1.

2. See Addendum 3 through the ISO NWI (New Work Item) process and expedite technical work on it.

3. Coordinate the CGEM work with registration proposals to insure maximum compatibility.

It appears that CGM Addendum 1 will complete soon, and it will have intact the capabilities that CALS has been working to put in and keep in. The final Iso balloting occurred early in the year, 
and there were few significant changes as a result. This is as desired; NIST/NCSL wished the content to be stabilized and "frozen" and the processing wrapped up.

Most of the extensions identified by CALS requirements studies are addressed in Addendum 3. At the start of the fiscal year a formal ISO process was beginning, under the leadership of the NIST representative, to initiate Addendum 3 as an Iso project. This process went exactly on schedule, and with the desired results, and is just completing as this fiscal year completes. (NOTE: There is one last step, which has gotten delayed, but it is hoped that this is a formality.)

Slightly ahead of schedule a Working Draft for Addendum 3 has been produced, circulated for ISO comment, and the US has had a letter ballot and formulated extensive technical comments. Assuming adequate resource commitments and no serious dissension among the member nations, the project is on track. According to the schedule in the New work Item, final text should be available in April 1991.

There is a potential problem looming, however. NIST/NCSL cannot be sure, despite the apparent ballot results, that the European members of SC24 share the same priorities and are willing to commit resources sufficient to complete the work, with the required content, in a reasonable timeframe. This should become apparent at the SC24 meeting in October 1989. ASC X3H3 will have to continue to have a fallback or contingency - possibly quick domestic processing as a US standard - if it is estimated that the project might bog down in ISO.

In addition to working on the CGM addenda, the NIST/NCSL representative reviewed and coordinated with the CALS Graphical Registration proposals during this fiscal year.

\section{Recommendations}

Further work is required if the Addendum 3 project is actually to complete expeditiously (or at all). It has been principally the NIST/NCSL representative who has been keeping the work alive and on track in both ANSI and ISO. continued participation is essential.

NIST/NCSL will be monitoring and assessing the study work which is just commencing to define what, if any, $3 \mathrm{D}$ metafile requirements exist for effective support of the emerging product data standards (PDES and STEP in particular). 


\section{Activities in FY89}

The remainder of this report contains details of the working meetings and progress on the CGM addenda during this fiscal year. Attachments containing key documents are included at the end. The material in the next two subsections is condensed from previous final reports on this subject and is repeated here for reference.

\subsection{Motivation for CGEM}

The CGM standard upon which MIL-D-28003 is based, ANS X3.122-1986, was completed in 1986. It is a "least common denominator" graphical file interchange standard. That is, it provides a suitable basic picture interchange format for diverse application areas. Its scope and content were not derived from any particular application area, but rather more from the content of other general purpose computer graphics standards.

This expedited the processing of the standard, at the cost of efficiency of usage in some application environments. The CALS application areas of technical illustration, technical publications, and compound document exchange comprise such environments. While experience shows that even the current CGM is more efficient than specifications such as page description languages (e.g., Postscript) and engineering data specifications (e.g., IGES) for graphical interchange, nevertheless CALS requirements studies show that the efficiency and fidelity of interchange could be improved with a well designed set of extensions to CGM.

For these reasons an extension process was commenced in 1986 within ISO SC24 to extend CGM functionality as required by its more advanced metafile application constituents.

\subsection{Historical Overview}

Two addenda were officially commenced in 1986:

- Addendum 1: originally intended to support certain needs of GKS, and replace the non-standard specification in Annex $E$ of GKS (i.e., provide a GKSM, workstation session capture metafile, to replace Annex $\mathrm{E}$ of GKS);

- Addendum 2: originally intended to support the metafile requirements of GKS-3D, and replace the non-standard Annex E of GKS-3D. 
Due in part to the successes of the work done in previous years

for this task:

- ASC $\mathrm{X3H3}$ endorsed and supported the CGM extensions sought for CALS.

- The scope of Addendum 1 was expanded to include some of these extensions: internal symbol libraries, additional geometric primitives, and basic raster primitives.

- Addendum 3 was commenced, to extend CGM further as required by technical illustration, engineering drawing, and publishing environments.

- Requirements statements and baseline technical documents for Addendum 3 were produced within ANSI and circulated within ISO as well. These materials were largely derived from CALS requirements studies and CALS activities.

- A Metafile Reference Model was devised within ANSI, submitted to and accepted by ISO, with the result that the content and targets of the various addenda were significantly reorganized and redefined. Relatively uninteresting GKS-related content, which would have caused unnecessary and undesirable complication as a CGM addendum, was thereby split off and attached to GKS.

The impact of CALS participation in the ANSI and ISO work under this and previous contracts is significant. The NIST/NCSI representative was the document editor of CGM (both the ANSI and ISO documents), has led and continues to lead the CGM work within $\mathrm{X} 3 \mathrm{H} 3.3$," has led and continues to lead the US delegation at ISO metafile meetings, and is the Iso leader of the Addendum 3 study Period. Without this participation the CGEM work would have progressed much more slowly, and in fact Addendum 3 might not have happened at all.

At the start of FY89, Addendum 1 was well advanced in its processing and was expected to change little (see the detailed activity reports following, however). Addendum 3 was entering a study phase in ISo, during which the requirements were to be studied and agreed upon. This work was largely based on US work in the previous year.

The following list summarizes the requirements which were identified and designated as the scope of Addendum 3 at the start of this Addendum 3 study Period, which corresponded roughly with the start of this fiscal year.

1. Internal symbol libraries; 
2. Reference to and invocation of pre-defined external symbol libraries;

3. Advanced drawing capabilities, including:

- user defined line type;

- user defined hatch style;

- a number of additional line types;

- a number of additional hatch styles;

- several types of spline curves;

- conics and conic arcs;

- closed figure primitive;

- arbitrary clipping boundary;

4. A number and variety of fonts;

5. A completely new text model based on the work of Iso 9541, Information Processing--Font and Character Information Exchange;

6. Additional raster primitives (and associated attributes for image processing);

\subsection{Review of Goals for FY89}

The goals for the FY89 CALS CGEM project were:

1. Addendum 3: completion of the formal New Work Item (NWI) procedures for Addendum 3 within Iso and resumption of the technical work, including:

- endorsement of the need and content of CGM Addendum 3 by the CGM Extensions Study Period;

- production of the necessary NWI and supporting Requirements Document;

- initiation of SC24 NWI ballot and processing of results;

- technical work on the initial draft of the functional content, which was a US contribution that was a year or so old.

2. Addendum 1: Finish it by: circulation of Addendum 1 DAD (Draft Addendum) text in $\mathrm{X} 3 \mathrm{H} 3$ and generation of $a$ US position on the SC24 DAD ballot; processing the SC24 ballot results and producing final text. 
3. Addendum 2 (3D): Prevent the project from draining resources from the Addendum 1 and Addendum 3 work until the project gets a well-defined and useful scope. This may in fact happen now as a consequence of liaison with product data standards committees within ISO. A 3D graphical file format may be defined to serve the needs of STEP/PDES. This would be a development of interest to CALS. However, the current Addendum 2 proposals are relatively unfocused and uninteresting.

\subsection{Key Activities of 1989}

These subsections, describing the key meetings and other activities during the task period, are organized first by addendum and then chronologically within each addendum section.

\subsubsection{Addendum 1}

\subsubsection{Initial status and Goals}

At the close of work in FY88 the Addendum 1 project had been split into two pieces: a static picture capture Addendum 1 to the CGM standard; and a dynamic audit trail metafile Addendum 1 to the GKS standard. This was in consequence of Metafile Reference Model work developed within X3H3.3/CGM (the US metafile group) under the leadership of the NIST/NCSL representative, and carried to the ISO SC24 meeting (Tucson, June 1988). The split separated the parts of interest to CALS - CGM Addendum 1 - from functionality to provide a GKSM capability for GKS (which is of no interest to CALS). At the same Iso meeting it was also agreed to skip a second round of PDAD (Proposed Draft Addendum) ballot and go straight to DAD status (DAD is the final processing stage for an Iso addendum). This was largely due to the position of the US delegation, which was being lead by the NIST/NCSL representative, and it saved a potential 9 month delay in completion of CGM Addendum 1.

At the beginning of work in FY 1989 then, the DAD text of CGM Addendum 1 was being circulated for a 6-month Iso sc24 ballot. With proper handling of voting and processing this ballot would be the last processing step for CGM Addendum 1 and its completion would be expected in late 1989 or early 1990.

\subsubsection{Houston X3H3 Meeting}

There was a meeting of the ANSI X3H3 committee in Houston, 17-21 october 1988. There was no activity of interest to CALS on the 
CGM Addendum 1 project at this meeting. The DAD text had not yet been received from ISO. There was only announcement and planning for an $\mathrm{X} 3 \mathrm{H} 3$ letter ballot on the DAD text when it arrived in late 1988 .

\subsubsection{DAD Ballot \& ANSI Letter Ballot}

The DAD text was received by the NIST/NCSL representative in December 1988 directly from the document editor in England. An X3H3 letter ballot was prepared and mailed with the CGM DAD.I text for a 30-day ballot, to collect and formulate the US position for the SC24 DAD ballot. The ballot period was 6 January 1989 to 6 February 1989. The DAD.1 document is similar to the text in Attachment 8 (Attachment 8 is the draft final text for CGM Addendum 1, which is the result of applying the DAD.1 ballot results to the DAD.1 text). Note that the GKS Addendum 1 document was being balloted simultaneously, but has not been included as it is of little interest to CALS.

\subsubsection{X3H3 Milpitas Meeting}

There was a scheduled X3H3 meeting in Milpitas, CA, February 610, 1989. The agenda for CGM work was mostly devoted to processing the comments from the DAD.1 letter ballot. The goal of NIST/NCSL for the US position was two-fold: there should be absolute minimal change in the DAD.1 text, to avoid forcing a second DAD ballot; and the CALS content of Addendum 1 should stay intact. A second DAD ballot at the ISo level would cause 9-12 months delay in the completion of processing. The Iso rules as traditionally interpreted by SC24 would require a second DAD ballot if there were any technical change.

One point in the US position deserves explanation. The US voted to remove PIXEL ARRAY from Addendum 1. This function (as reported in 1987) was one of the CALS functions and was supported by CALS requirements studies. The NIST/NCSL representative supported the removal because the function had been formulated in a way which did not meet CALS requirements. The function was taken straight from the CGI (Computer Graphics Interface) standard, and had a parameterization which was device dependent, could not be coordinated with the other geometric primitives of CGM, and was conceptually different from the PEL ARRAY GDP which had been promoted for Graphical Registration.

This technical deficiency alone was sufficient reason for removal of PIXEL ARRAY. Opposition and negative votes were anticipated from at least UK and France as well. 
Attachment 1 contains the US vote and comments on the Iso ballot, which was formulated at the Milpitas meeting under the leadership of the NIST/NCSL representative. Note that the PIXEL ARRAY comment urges that it be reformulated and properly included in Addendum 3. Note also that the US voted "no". This is procedurally required by ANSI if a technical (as opposed to editorial) comment is being made. As delegation leader to the ISO editing meeting on Addendum 1, the NIST/NCSI representative was empowered to change the US vote to "yes" if the US comments were adequately addressed at the meeting.

The ISO DAD ballot was scheduled to end 15 June 1989, and results were scheduled to be processed at an SC24/WG3 MRG (Metafile Rapporteur Group, the sub-group of WG3 responsible for CGM maintenance and CGM extensions) editing meeting 20-25 June 1989 in Waikoloa, HI.

\subsubsection{Between Milpitas and Waikoloa}

Private communication by electronic mail took place between the NIST/NCSL representative, who is the principle US metafile delegate to the SC24/WG3 MRG, and the principle metafile delegates of the UK and Germany from February through June. It became clear that there were a number of "no" votes on the CGM DAD.1 text, and there were a number of technical issues. This raised the possibility that technical changes would force another DAD ballot. The UK delegate thought it inevitable. The US thought it must be avoided at all costs, in part because the SC24 metafile workers had to be freed up to work on Addendum 3 . Germany agreed with the US. The UK finally agreed that the US could make technical changes and avoid a second DAD ballot if there were consensus on all of the changes as per Iso rules. For this reason the US circulated and debated the various national positions quite a bit during the months before Waikoloa, and were in substantial agreement before the meeting commenced.

\subsubsection{Waikoloa MRG Meeting}

The MRG met in Waikoloa, HI, 20-25 June to process the results of the DAD ballot. The US delegation consisted of one person, the NIST/NCSL representative. The goal was to resolve all negative comments and get unanimous approval, amend the document as agreed, and thereby finish work on CGM Addendum 1 .

The initial count on the DAD ballot was: 8 approve, 5 disapprove. Disapproving were Austria, France, Germany, UK, and US, in other words all the key countries. The minutes of the meeting are not yet available, so cannot be included with this report. A summary is presented below. 
The negative comments fell into a few categories:

- Unhappiness with PIXEL ARRAY (nearly unanimous);

- Divergence between the specifications of Addendum 1 and CGI where they overlapped (much of the functionality of Addendum 1 was borrowed from CGI and the two standards were to remain identical);

- Misunderstanding of the meaning of some of the items in Addendum 1 (a number of French comments fell into this category).

The biggest problems during the six days of issues reconciliation and editorial work came from attempting to keep Addendum 1 and CGI (and CGM itself!) identical where they overlap. CALS does not consider CGI an important standard at this time; however, in the original model of the relationship between the standards CGM is basically identical to the output functions of CGI. Many still believe that this is a critical principle, and that abandoning it will impose unnecessary burdens on the computer graphics industry and other industries which rely on graphics standards. So effort is always expended to keep the two together. However, there seem to be those working on CGI who have forgotten this principle or no longer believe in it, and this creates conflicts such as occurred at the waikoloa editing meeting. The CGI committee was meeting in parallel to process the results of its 2 nd DP ballot and attempt to advance it to DIS stage.

The worst of the compatibility problems came when it was realized that CGI had changed the method by which clipping behaved under the copy segment function and its transformation. This issue is important to CALS, because one of the main pieces of functionality of interest to CALS is the Global segment feature which was built into Addendum 1. In some cases (the default case in fact) the new CGI model had a tedious and complex scheme whereby clipping rectangles could accumulate and become arbitrary convex polygons. This is not the way CALS wants to use Global segments. The CGM committee was unanimous in not wanting to have this functionality. After much debate with the CGI committee, the best compromise that could be achieved was: repackaging the functionality in such a way that an application profile can easily exclude the features that it does not want.

As a result of the editing meeting, were resolved on CGM Addendum 1 . participated in the editing meeting, France and Austria. The remaining based on a single technical objection. all negatives but Austria's Germany, UK, and the US with occasional input from negative from Austria was 
The editing meeting produced markup for complete final text, and the document editor (Anne Mumford of UK) will have final text around late August.

France wanted a second DAD ballot because of the changes that were made. The US, with support of UK and Germany, proposed a methodology that is somewhat unconventional for SC24 but would save another ballot round. The five nations represented at the CGM meeting would agree that the Addendum was acceptable with those modifications agreed to at the meeting, and the document editor would circulate the document for a six week review to verify that the agreed changes were properly implemented. After that review the document would go to ISO Central secretariat with the recommendation that it be made an international standard. It is hoped that the secretariat will progress the document, but this is not certain because there is an outstanding negative (Austria).

Except for PIXEL ARRAY, no significant functionality has been added to or deleted from Addendum 1 , but some existing functionality was repackaged. The processing of comments resulted in the following changes to CGM Addendum 1:

1. PIXEL ARRAY is removed; improved raster capabilities will be revisited in Addendum 3 and treated in a coherent manner.

2. METAFILE CATEGORY is removed; the current basic_static category is preserved by allowing "VERSION 1" to appear in the Metafile Descriptor. Annex $A$ is corrected and made into a new normative Annex $J$ (to define VERSION 1).

3. In a repackaging of functionality, clipping is removed from INHERITANCE FILTER; the exact same clipping features are now selected by a new function CLIPPING INHERITANCE.

4. The IMPLICIT EDGE VISIBILITY (a feature of Closed Figures) was discovered not to work properly, so was removed and its functionality replaced by CONNECTING EDGE.

5. A new datatype NAME, with its own NAME PRECISION, will replace datatypes $\mathrm{ASN}, \mathrm{PN}$, and $\mathrm{SN}$.

Although GKS Addendum 1 (the other half of the original CGM Addendum 1 after the split at Tucson) is not of interest to CALS, there was one result on its processing which is related to the CGM Addendum work. The UK took the position that a standard GKSM is not needed, and that its standardization will confuse the marketplace. They wanted the project dropped altogether. Although sympathetic, the US would not vote this position because of agreements made earlier with GKSM supporters to support the work (in return for them not holding up CGM). 
Instead changes were made to GKS Addendum 1 to make it conform much more closely to the GKS notion of a session-capture metafile, and to make it much more distinct from CGM (and hopefully less likely to be confused with CGM in the real world). The delimiters of the GKSM (GKS Addendum 1) were renamed, to reduce the chances of metafile interpreters confusing a GKSM with a CGM. In particular, the BEGIN METAFILE element was renamed BEGIN GKS SESSION METAFILE, and given a unique encoding. Similar changes were made for BEGIN PICIURE and END METAFILE. BEGIN PICTURE BODY and END PICTURE were removed. Thus in any encoding the GKSM becomes structurally different and is introduced by different delimiters.

one final note of interest for the waikoloa meeting: this was the last meeting for Eckhard Moeller, of Germany, the rapporteur of the WG3 Metafile Rapporteur Group. Anne Mumford of the UK assumes the rapporteur position starting at the Brazil meeting. The NIST/NCSL representative could probably have had this position. However, chairing the group compromises the chair's ability to influence the technical content of the work. The latter role is more important for CALS, on the Addendum 3 work, on which the MRG should henceforth be spending most of its effort.

\subsubsection{Nashua X3H3 Meeting}

An X3H3 meeting took place in Nashua, NH during the week of september 25-29, 1989. There was no significant activity on Addendum 1 at this meeting.

\subsubsection{Status \& Remaining Work}

The revised CGM Addendum 1 document, the draft final text, was received directly from the document editor in England in mid-September. Some 20 copies have been distributed to US experts for final review and accuracy checking.

It is clear that some parts of the text have not been completed as evidenced by the document editor's cover memo. In addition, a new annex containing a formal grammar was overlooked altogether. For these reasons, the text may have to be circulated again after these parts are supplied. If this is not done, then final text will not be reviewed by anyone except the document editor. This decision to circulate will be made at the SC24/WG3 MRG meeting in october. If another round of review is required, the final acceptance of the text will be delayed by another 2 months. 


\subsubsection{ANSI Processing of Addendum 1}

As shown in the preceding sections, all work that has occurred is ISO work towards an Addendum to the ISO version of CGM (ISO 8632), with all US activity contributing to that work. MIL-D-28003 is based on FIPS PUB 128, which is identical to the US version of CGM (ANSI X3.122). The US and ISO versions of CGM are in fact identical in content, but differ in editorial style.

An addendum (see Attachment 8 ) is an editing script. As such, it is not a complete standalone document but consists of change directives against the base document. To adopt Addendum 1 in the US would require a document editor to revise the Iso text.

Consequently, ANSI has devised new procedures which make it much easier to adopt ISO work automatically as ANSI standards, and to designate ISO standards as ANSI standards. The NIST/NCSL representative is seeking such a change on status for ANSI CGM. Thus, the current ANSI document would be withdrawn and replaced by the ISO document. The ANSI and ISO versions of CGM would then be the same document. After this change, ISO Addendum 1 could be adopted directly. The implications on MIL-D-28003 should be minor, since it would only have to be checked to make sure that page references are correct, and that the proper base document is referenced.

\subsubsection{Addendum 3}

\subsubsection{Initial status and Goals}

At the start of FY89, the US had been working on Addendum 3 project for a year. In prior CALS work, the metafile requirements of CALS which were not met by CGM and not met by Addendum 1 (or Addendum 2) were identified. An Addendum 3 was proposed, and its approximate contents were defined. A strategic choice was faced: should this be expedited as an ANSI project, or should it be pursued as an Iso project? Although the former would probably be more expeditious, past experience has shown that if ISO undertook such work, and if it did so before ANSI completed, then it would be likely that the ANSI work would be put aside and ANSI would join the Iso effort. For this reason, it was decided to pursue the work aggressively in Iso.

The US first proposed the project at the initial Sc24 meeting in December 1987. The project was not accepted at that time, 
because SC24 procedures and priorities were in flux, and there was some dispute within ISO about which committee should lead on the Addendum 3 content. Consideration of the project was deferred to a special study meeting of SC24 procedures which took place in April 1988. Primarily due to lack of time, the meeting did not address Addendum 3, but the NIST/NCSL representative did get the point across that the US would undertake the project on its own if ISO did not move on it. At the SC24 plenary in June 1988 a study group was established, lead by the NIST/NCSL representative, to initiate the project under new SC24 procedures, get consensus on its scope, and coordinate with parallel study groups for Improved Graphical Text Model and for Product Data Geometry (PDG).

The parallel study groups were formed because their two technology areas would be important for all new graphics standards. The text model of all first generation standards (CGM, GKS, PHIGS, CGI) was acknowledged to be inadequate for modern typographic, engineering, and presentation requirements. The purpose of the text study group was to identify a new common model for the second generation of standards, which would include Addendum 3 .

The PDG group was formed for a similar reason. The set of geometric primitives in most first generation standards was rudimentary. CGM was better, including some conics, etc. But modern practice requires a more substantial set, including such primitives as various sorts of splines and full conics. The PDG group was charged with defining the relationship between product data standards (PDES, STEP, etc) and graphics standards. From this relationship might be deduced further geometric primitives which should be in the graphics standards in order to fully support product data standards.

The Addendum 3 study group was to report at the next sc24 plenary, October 1989, and was to complete all of the required New Work Item (NWI) processing steps and produce a Working Draft (WD) Addendum 3 by then. The initial meeting of the study group was scheduled at the beginning of FY89, and was arranged so that the two technology groups (Text and PDG) would meet immediately before the Addendum 3 group at the same location.

\subsubsection{Redondo Beach Text Meeting}

An ad hoc meeting of several US experts, including the NIST/NCSL representative, was held December 1-2, 1988 in Redondo Beach, CA. The purpose of the meeting was to write a position paper for the upcoming meeting of the text study group. This paper listed all the features that an improved text model should have. It may be found in Attachment 2 of this report. 


\subsubsection{Munich Study Group Meeting}

The first set of study group meetings was scheduled for January 16-20, 1989 in Munich, West Germany. Text and geometry groups were to meet in parallel during the first half of the week, and CGM extensions (Addendum 3) during the second half. Thus, metafile experts would be able to participate in one of the technology meetings, and the technology specialists would be able to participate in the CGM extensions meeting. The meetings were held as scheduled, despite last minute attempts of PDG to move its meeting to a later date. The NIST/NCSL representative, who is the leader of the CGM extensions group, was unable to attend due to a family emergency. Peter Bono, chair of $\mathrm{X} 3 \mathrm{H} 3$, chaired the meeting in his absence.

US input to the CGM extensions meeting consisted of a draft New Work Item (NWI) proposal and supporting material for producing a Requirements Document (these were listed as base documents in the meeting call, which was written and submitted by the NIST/NCSL representative). The supporting requirements material included previous CALS requirements studies and parts of earlier Addendum 3 proposals.

The meeting resulted in the group endorsing the need for Addendum 3 , and advising that it be expedited. A draft NWI and Requirements Document were produced as required by new SC24 procedures. These were substantially the same as the input documents - all of the important CALS requirements were endorsed and not many more were added. Details can be found in the attachments.

Several milestones in the NWI schedule should be noted:

1. SC24 ballot on the NWI and Requirements to complete by the June 1989 Waikoloa meeting;

2. processing results at that meeting;

3. JTC1 ballot to complete by the october 1989 SC24 plenary;

4. substantially complete working Draft of Addendum 3 to be done by the end of that meeting;

5. final text for Addendum 3 in April 1991.

The minutes of the Munich meeting are found in Attachment 3 of this report. Attachment 4 contains the final NWI and 
Requirements Document for the project. These were actually produced at the Waikoloa meeting in June 89 , but included here because they are so similar to the drafts from the Munich meeting that the difference is negligible (there are a couple additions to the bibliography and a couple additions to the list of liaison projects).

The output of the meeting also included three 1-page letters, to the rapporteurs of the SC24/WG1 Reference Models group, Requirements group, and the rapporteur of SC24/WGI himself. These letters basically asked the recipients to carry out the NWI processing steps required under the new SC24 procedures.

\subsubsection{Milpitas X3H3 Meeting: Liaisons and Drafting}

There was a scheduled X3H3 meeting in Milpitas, CA, February 610, 1989. The agenda for CGM work was mostly devoted to processing the comments from the CGM Addendum 1 letter ballot (see the above section on Addendum 1). There was little time for Addendum 3 work. However:

- there were extensive liaison meetings between CGM people and experts from other technology areas: text, CGI, geometry, image storage, etc.

- the NIST/NCSL representative leading the CGM meetings made interim writing assignments to the $\mathrm{X} 3 \mathrm{H} 3.3 \mathrm{CGM}$ people with the goal of assembling a new Addendum 3 baseline document prior to the Waikoloa meeting.

\subsubsection{Between Munich and Waikoloa}

Many of the details of processing the Addendum 3 NWI during this period are found in the final report of the rapporteur of the CGM Extensions Study Period (the NIST/NCSL representative), which is in Attachment 5. To summarize briefly:

1. As per the new SC24 procedures, the NWI and Requirements Document went to the SC24/WGl Reference Model meeting the week following the Munich meeting. No changes were requested by that group.

2. As per the new SC24 procedures, the NWI and Requirements Document went to the SC24/WG1 Requirements rapporteur for their late February meeting. That meeting was cancelled and so no changes were requested by that group.

3. The SC24/WG1 group at its February meeting endorsed the Munich results and asked the SC24 secretariat to circulate same immediately for a SC24 NWI ballot. 
4. This was done, and the ballot closed June 15, 1989.

In order to form the US position on this ballot, the NIST/NCSL representative prepared an $\mathrm{X} 3 \mathrm{H} 3$ letter ballot. This was sent to the $\mathrm{X} 3 \mathrm{H} 3$ mailer in early April for a 30-day ballot period. Mailing problems with that $\mathrm{X} 3 \mathrm{H} 3$ officer resulted in the ballot being delayed until early June. This was too late, so the ballot was scrapped and the US voted "yes without comment" on the NWI.

In this period, the NIST/NCSL representative had to prepare and circulate the call for the next CGM Extensions study Period (Addendum 3 study group) meeting. SC24 procedures require that this be done at least 2 months in advance of the meeting, which was scheduled for Waikoloa in late June.

Finally in this period, the NIST/NCSL representative coordinated the assembly of the Addendum 3 draft by X3H3.3 CGM people. In early June this draft was mailed to the international attendees of the Waikoloa meeting.

\subsubsection{Waikoloa Meeting}

The final scheduled meeting of the CGM Extensions study Period took place in Waikoloa, HI, June 26-28, 1989. This was immediately following the CGM Addendum 1 editing meeting. The meeting was chaired by the NIST/NCSL representative. The goals were:

- process the results of the NWI ballot;

- revise the NWI and requirements if necessary and send to the SC24 Secretariat for immediate JTC1 ballot (JTC1 is the parent organization of SC24);

- continue technical work on the baseline document;

- begin work on the final report of the study period.

All of these goals were met. The study period final report in Attachment 5 contains details. This final report was actually produced subsequent to the meeting.

One point in the final report deserves note. It is clear that SC24/WG3 MRG is facing a resource crunch. After all of this work to get the project through the ISO procedures, the question of 
whether ISO has the resources to deliver the project on schedule must be seriously looked at. This will be determined at the october meeting. The US will need to develop a contingency strategy in case Iso cannot deliver.

\subsubsection{Post Waikoloa \& ANSI Letter Ballot}

The work done at Waikoloa on the Working Draft of Addendum 3 was incorporated by the document editor. This document is contained in Attachment 6. In late July, it was sent to the SC24 Secretariat for SC24 circulation and comment. This is normally a 3-month period, but early comment had been requested so that technical work may take place at the SC24 plenary 15-30 October 1989. An X3H3 letter ballot had been drafted and circulated as well. The purpose was to solicit comments on the Working Draft of Addendum 3 and use them during the X3H3 meeting of september 25-29, 1989 in formulating the US position.

It has since been learned, that the JTCl ballot period had not even commenced, although the Addendum 3 schedule showed it being complete by the SC24 meeting in october. The reason for this delay is disorganization within the JTCl secretariat and lack of communication between it and the SC24 Secretariat. The consequence is that Addendum 3 is still not an official Iso project. Although JTCl approval is usually considered a formality it is nonetheless the last required step in the process.

It is also at the JTC1 voting stage that the member nations formally indicate their levels of participation and of resources. This will be a key issue to the success of the project in SC24.

\subsubsection{Nashua X3H3 Meeting}

The X3H3.3 CGM subgroup had 6 attendees at the Nashua meeting, 25-29 September. With the exception of a two-hour liaison meeting with $3 \mathrm{D}$ and product data experts to define a response to an outstanding Addendum 2 ballot, the entire meeting was devoted to processing the substantial X3H3 comments on the Addendum 3 Working Draft (WD) and forming a US position for the ISO comment period.

The US position is contained in Attachment 7. In summary, the WD has significant omissions and in other respects is very rough. This is to be expected at this stage in the standards process. The WD will require significant improvement, however, before the first official voting can commence in ISO. This is the PDAD ballot. It is possible that these improvements can be made at the SC24/WG3 meeting in October. 
The US position also makes clear what the priorities of the work should be. Private communications with other national delegates indicate the possibility of other prioritizations which may seriously slow the project (see next section).

The key technical problems can be briefly summarized:

1. the geometric primitives are incomplete with respect to what was specified in the NWI and Requirements documents;

2. the formulation of some of the geometric primitives, as well as the image primitives, needs improvement;

3. numerous technical and editorial details concerning the additional attributes, color models, etc need to be corrected;

4. the improved text capabilities need a thorough overhaul, including: clarification of how external font resources are accessed; better glyph access methods; clarification and improvement of font callout and substitution techniques; removal of glyph definition primitives from the metafile.

\subsubsection{Status \& Remaining Work}

It appears that the Addendum 3 project has been accepted by ISO, but the final round of balloting in JTCl has not yet taken place. There is a procedural possibility that this can be skipped, that SC2 4 can issue a resolution that this is a simple revision of an existing standard and so JTCl (SC24's parent body) need not vote on it. This will have to be explored at the october SC24 meeting.

Although the formal acceptance seems likely, there is still danger that the project will not progress adequately. This comes from views of some of the other national delegations about metafile extensions. The US view is that CGM relates to other computer graphics standards, but relates more to "current practice" in engineering, publishing, and graphics arts. This means that users in these areas are retro-fitting CGM import and export filters to existing proprietary products. In the process, they care little about the other work of SC24; they just want to exchange graphical information between heterogeneous systems. In other words, in the view of US users, Addendum 3 has more to do with IGES, PDES and Postscript than it does with GKS. 
The view in Europe tends to be less pragmatic and more academic. There is high interest in Europe in a new Application programmer Interface standard (API) - a revision to or replacement of GKS. Comments have already been heard and seen to the effect that the Addendum 3 work must go no faster than the new API work, that they must work out access to all new technology in compatible (meaning identical, to the commenters being referred to) ways. These sentiments seem to echo similar conflicts that occurred during CGM standardization, and which slowed the standard down by 1-2 years.

There are also indications that some think the scarce resources of SC24/WG3 should be split between Addendum 3 and Addendum 2 (3D). NIST/NCSL believes there are barely sufficient resources to do the Addendum 3 work. Any attempt to carry on Addendum 2 as well, without additional staff, would have serious impact.

The US may thus face an important and strategic decision in the october SC24 meeting: should it continue to support the Addendum 3 work as an ISO project; or should it attempt to block further Iso work on metafiles for a couple of years and go back to ANSI processing? The latter is clearly a drastic resort. It has been the US position that the work belonged in ISO. IsO endorsed this, and the tentative schedule, in the NWI process. US companies definitely will fare better in international markets if their standards are international standards. However, if the other ISO member nations do not actually have the will to follow through with the commitment to constituency and schedule that was indicated in the NWI process, then the US must consider withdrawing the project.

\subsubsection{Addendum 2}

Since the Waikoloa meeting a version of the Addendum 2 (the 3D addendum) document has been circulated for PDAD ballot within SC24. The US had to respond to this ballot. So a second $\mathrm{X} 3 \mathrm{H} 3$ letter ballot was put together and circulated. This was processed at the september meeting in Nashua.

The US position was defined at the Nashua meeting in liaison with PHIGS experts and those interested in product data standards (STEP/PDES). It appears that the PHIGS extension project in X3H3 will take interest in this project, as a means of providing support for STEP, and will help to define a useful scope. There is the possibility of additional staff and help from the 3D subgroups, in which case there could be sufficient staff to process both Addendum 3 (which is important to CALS), and Addendum 2 (which is not). 
These points are speculative, however, as the necessary preliminary liaison between the 3D experts and the product data standards committees has not yet occurred. Accordingly the US position on the Addendum 2 PDAD ballot contained four simple points: no further effort should be expended on the Addendum 2 as currently specified; the need for a 3D metafile should be defined by 3D experts and metafile experts in liaison with TC184 (the ISO committee working on STEP); any resulting metafile project should be jointly executed by $3 D$ and metafile experts; WG3 must have additional resources to execute its part of any resulting project. Should a 3D metafile project result, it is anticipated that the $3 D$ experts would principally define its functional and semantic content, as well as its position in the reference model, and metafile experts would design the metafile and write its encodings.

4.4.4 Coordination between CGM Addenda and Graphical Registration

NIST/NCSL has been sponsoring registration of graphical items for CALS. These are intended to provide a short term solution to functions needed by CALS that are being pursued through the slower formal standards process (Addendum 1 and Addendum 3 ).

Because these are addressing the same needs, the formulations in Graphical Registration and the addenda should usually be very similar (there are cases where the different mechanism of the GDP and ESCAPE elements which are registered justify some difference in formulation).

During this fiscal year there has been frequent liaison between the NIST/NCSL representative and NIST/NCSL to coordinate the content of CGM Addendum 3 with the registration proposals. The results have been adjustments to proposals in Registration Batch 2 and Registration Batch 3 (see the final report titled FINAL REPORT, CALS FY89 SOW TASK 4.3.2, MIL-D-28003 REVISION RECOMMENDATIONS) as well as reformulation of Working Draft Addendum 3 and additional specifications for the pending revision of MIL-D-28003. The effect of the adjustments is generally a convergence of the proposals and the draft addenda. 
Attachment 1:

US Vote and Comments on CGM Addendum 1 DAD Ballot 



\section{U.S. Comments on ISO 8632-1/DAD.1 (CGM Add.1)}

The U.S. disapproves ISO 8632-1/DAD.1 with the following technical comment:

PIXEL ARRAY should be removed from CGM Addendum 1 and should be considered as part of the Addendum 3 project. There are several reasons: 1) Image storage and transfer capability is being studied for Addendum 3 and the PDXEL ARRAY capability should be included in this more comprehensive study; 2) The current PIXEL ARRAY formulation is based on the CGI formulation and the latter is considered unstable at this point; 3) The current formulation of PLXEL ARRAY is device dependent and apparently does not exist in the reference model at the same level as other CGM elements; its relationship to the other elements at least needs to be more carefully defined before being included in CGM extensions.

In addition, we note the following inconsistancy with the 2nd DP tert of CGI and request that this inconsistancy be addressed jointly by the CGM and CGI RGs of WG3:

The behavior of CLIP RECTANGLE under COPY transformation differs between the CGI and CGM. We believe the CGM specification is more compatible with API standards. We understand that the CGI specification is still subject to change in this area. In any case, this must be resolved between CGI and CGM.

\section{Editorial Comments:}

E1: The discussion of the effects of anisotropic transofrmation in 4.12 .45 has been clarified in the CGI. CGM should adopt the clarified wording.

22: Section 4.124.4 should point out that SEGMENT PICK PRIORTY has no graphical effect and is avaliable for application dependent communication between interpreters and generators. The same should be pointed out for PICK IDENTIFIER in section 4.7.9.

E3: In Sections 4.125 and 5.10.12 PICK IDENTIFIER and ASF's have been omitted from the description of DNHERITANCE FILTER. CGM is intended to be the same as CGI in this area.

E4: Page 1, sub-clause 0.3, item c) should be deleted. It is not possible to anticipate what future standards will require. In any case, it does not add any useful information to the standard.

E5: Page 41, Table 3.1. The entries for SCALING MODE are wrong. The entire table should be carefully checked for correctness.

E6: Pages 56-57, sub-clauses 5.42-5.45. Reword these three sub-clauses along the lines of, The COLOUR SELECTION MODE may be changed only within the picture description in category "basic-static". It may be allowed in the picutre body in some of the other categories. 
58: New sub-clause 55.15. There seems to be a remoant of an old version (stacked attribute sets) indicated here. If attribute sets are named, is it not the case that the NAMED attribute set be the one restored, not the LAST one?

E9: Page 103, new element defaults. SEGMENT DISPLAY PRIORITY and SEGMENT PICK PRIORITY should not be encoding dependent, but rather the default should be zero.

E10: The grammar has not been carefully reviewed in the past and we request that a careful examination is done before IS tert is produced.

E11: For Note 1 under H 6.7, "action required" flag and "no-action" do not seem to be referenced anywhere else in the document.

E12: There is an inconsistancy in the usage of phrases "view surface" and "display surface". To be consistant with itself and with CGI, the phrase "drawing surface" might be a better choice.

E13: All references to a CGM "function" should be replaced by "element".

E14: In the definition of anisotropic mapping, we suggest replacing "to physical device units" with "distance units on the physical drawing surface".

E15: In the definition of edge, we suggest replacing The rendering of the boundary" with "The rendering of the perimeter" to avoid confusion about interior style HOLLOW (rendering of the boundary) versus EMPTY with edges visible (rendering of the edges or perimeter).

E16: In the definition of isotropic mapping, we suggest replacing "device coordinates" with "distance on the drawing surface".

E17: In the definitrion of size specification mode, we suggest replacing "the state list" with "the Modal State List". Use of the terms "state list" and "current state list" need to be looked at. The concept of "Modal State List" was introduced in clause 4.1224 More needs to be said about this concept earlier so that it can be used and refered to where needed. Also, more could be said about the general states of the metafile interpreter. Table 3.1 is great, but there needs to be some more general discussion.

E18: The definition of graphic object should be added to the definitions. It is used in many places, e.g the object clipping mode concepts. Also, we suggest using only the term "graphic object" and not variations such as "graphical object".

E19: In clause 5.4.9, the parameter should be called "device viewport specification mode".

E20: Page 42. sub-clause 5.1 Abbreviations. The meaning of PN should be:

PN Pick Name Pick Identifier

Realization is an integer.

Range is implementation dependent.

521: Page 12, sub-clause 4.2 . The reference should be to sub-clause 4.4 .2 
E22: Page 2, 6th paragraph, "compound teat": Change "text that contains" to "tert that may contain" (a compound tert string need not contain attribute changes, it's compound if it was specified with multiple texa/append text elements).

E23: Page 4, Section 4325: This should be cailed something other than "gksm" now. We suggest "Add.1-static-gks." GKSM should be reserved for the audit trail that is described in the other (GKS) addendum.

524: Page 6, 4.3.43, end of 4.4 paragraph: It is very confusing, perhaps inappropriate, to keep refering to categories which are not static picture-capture metafiles. There aren't any defined in this addendum. Standards should not be written in such a way that they imply or assume very much about future extensions. There should be a single paragraph that says "Various restrictions (such as where elements are permitted) are permitted to be different in categories to be defined in future extensions, or in metafiles defined in other standards which are based on this one," and leave it at that.

E25: Page 7, Section 4.4.7, end of 3rd paragraph: "relative to the non-inverted viewport" does not convey the necessary information. 5.4 .10 has the proper wording and it should be repeated here or referenced here.

E26: Page 8, paragraph 7: Under LOCUS THEN SHAPE, it should also be noted that a thick line whose locus is outside of the clip window will not have any portion visible even if its line width would earry some portion of the rendering into the clip rectangie (same as LOCUS clipping).

E27: Page 8, Section 452, paragraph 8: 'When a width or size specification mode is 'scaled', the rendering of shape proceeds in DC space after the VDC-to-Device Mapping." It is unclear whether this simply applies to the anisotropic_mapping_and_wide_lines question, or whether this is implying that SHAPE CLIPPING doesn't work with sealed specification modes. Without using CGTs pipeline, much of the wording is unclear. SHAPE CLIPPING dips the same regardless of the specification mode (that the whole point), and the wording simply needs to be clarified.

528: Page 15, Section 4.1245, 4h paragraph from the bottom, last sentence: Since the segment transformation is VDC->VDC, the VCD->DC mapping (set up by VDC EXTENT and DEVICE VIEWPORT etc) is applied afterwards. The last sentence of this paragraph could be read as meaning that the latter transformation is only applied if the SEGMENT TRANSFORMATION was not applied. We think the work "only" is needed after the word "using"

529: Section 432.4 and 4325 refer to "GKS". Please change this to the "IS \#-year" form of reference.

E30: 4.123.1. Each segment has a unique identifier." This is not exactly what is intended. We advocate No two global segments may have the same identifier and no local segments may have an identifier the same as other local segment in the same picture or the same as a global segment."

E31: 4.1232. This clause does not actually state how a segment is opened. More generally, it seems sloppy to use the GKS words of "OPEN" and "CLOSED" to refer to static picture capture CGM files. It seems more natural to use terms like "elements delimited by a BEGIN SEGMENT element and an END SEGMENT element" when talking about CGMs.

E32: Defining a local segment in a picture automatically includes that segment in the picture's image. This needs clarification. 
E33: What are the highlighting, pick priority, and display priority for primitives outside segments? This needs clarification.

1234: 5.10.1.2, 6th line. To what does "(see below)" refer?

E35: Under "Page 6 clause 3" object clippi I mode needs to specify what "LOCUS" and "SHAPE" clipping imply and how they differ. "LOCUS THEN SHAPE" appears to be the logical concatenation of the other two modes. Also, the definition of "global segments" should read "these are segmeats which..."

536: Page 10, sub-clause 4.3 Note. Use "within the definition of a global segment" rather than the present "when" construction.

E37: Last addition to Page 10, sub-clause 4.3: Finish first sentence with "in a metafile of any category other than..."

E38: Clause 4.3.4.1 should re-iterate that only a metafile of category "basic-static" is premitted to omit the metafile category element as impled by the first paragraph of Addendum 1, page 3 (sub-clause 43).

239: Clause 4.4 first paragraph: Strike the last sentence or rephrase. All IS 8632 metafiles (regardless of Addenda work) are static picture-capture metafiles.

E40: Page 14, 4.4.7 rephrase, since no CGM categories may be other than static picture-capture metafiles. See also item 7 .

EA1: Page 14, 4.4.8 same as 9 above. See also item 7.

E42: Page 15, sub-clause 452, 3rd paragraph does not adequately explain the difference between "LCUS" and "SHAPE".

EA3: Page 15, sub-clause 455, paragraph 7 does not adequately explain bow LOCUS THEN SHAPE" may produce any difference from "SHAPE" alone.

EA4: Page 15 sub-clause 4.6 There are several lists in the sub-clause. To which one(s) should the element be added?

545: Page 20, 4.6.8.1. The phrase "A closed figure is opened.." is worded too mucis iike segments. Use "started" rather than "opened". Likewise, use "finished" rather than "closed" for END FIGURE.

E46: Page 20, 4.6.8.3 - State explicitly whether the seguence "New Region; End Figure" is valid.

547: Page 40, 4.1221 Provide a reference to 4.125 for bebavior of COPY SEGMENT.

E48: Page 50 subclause 53.11 - The existing shorthand names do not include hyphens, even for multiple word names. Shouid the addendum bave them?

E49: Page 58, sub-clause 5.4.6 - elimitate the "double negative" for clarity.

E50: Section 4.1222. Here and elsewhere, references are made to CGM states not included in Table 3.1. In particular, this sections mentions state GSD which is not in Table 3.1. 
551: Section 4.1222 The last sentence in this section implies that only the stated elemen" are allowed in the segment. This is clearly not the case.

E52: Section 4.125 In the example, the multiple attribute changes described by the right column for a single COPY SEGMENT (2) instance should be more explicitly mapped to actions which are taking place. More explanation is needed to clearly illustrate which actions in the segment being copied actually cause the change of state. 


\section{U.S. Comments on ISO 8632-2/DAD.1}

The U.S. disapproves ISO 8632-2/DAD.1 with the following technical comment:

The technical changes to ISO $8632-1 / \mathrm{DAD} .1$ must be reflected in this part .

\section{Editorial Comments:}

None. 


\section{U.S. Comments on ISO 8632-3/DAD.1}

The U.S. disapproves ISO 8632-3/DAD.1 with the following technical comment:

The technical changes to ISO 8632-1/DAD.1 must be reflected in this part .

In addition, we note the following inconsistancy with the with the proposed binary encoding of CGI and request that this inconsistancy be addressed jointly by the CGM and CGI RGs of WG3:

CGM and CGI Ire inconsistant in the specification of precision of integers representing the dita types SN, PN, and ASN. The CGI specification uses fixed sized 16-bit integers, which limits each of SN, PN and ASN to 64K unique identifiers. CGM uses integers subject ot integer precision.

\section{Editorial Comment:}

Clause 7.10, COPY SEGMENT. The enumerated values do not follow the null-value rule that is used in CGM. They should be:

$0:$ no

1: yes.

Page 17, new item b). Why is metric scale factor allowed to use fixed format real when sealing mode is not? The text should highlight this difference. 


\section{U.S. Comments on ISO 8632-4/DAD.1}

The U.S. disapproves ISO $8632-4 / D A D .1$ with the following technical comment:

The technical changes to ISO 8632-1/DAD.1 must be reflected in this part .

\section{Editorial Comments:}

None. 
Attachment 2:

US Contribution to the Improved Graphical Text Model 

ISO

International Standandization Organization

Organization Internationale de Normalisation

\section{ISO/IEC JTC1/SC24}

Computer Graphics

Secretariat: FR of Germany (DIN)

Tile: os Contribution to the Improved Graphical Text Model Study

Source (Country, Organization, Committce): aNSI

Status: $\square$ SC24 Output Document

Member Body position

Expert opinion (ALl expert contributions submitted to SC meeting must be authorized by the Member Body).

$\square$ Working Group output

Type: $\square$ Al:"Immediate" Output Document from WG/SC24 Meetings (to arrive at SC24 Secretariat 2 weeks after meeting).

A2: "Delayed" Output Document from WG/SC24 Meetings (10 arrive at SC24 Secretariat 4 weeks after meeting)

B: National/Exper Contributions to WG/SC24 Meetings (10 arrive at the WG or SC Secretariat 8 weeks before the beginning of the the WG or SC meeting)

C. Recent Liaison Documents (to arrive at the expert by the start of the WG or SC meeting)

Type of Distribution: Through SC24 Secretariat Direct to Member Bodies

Date of Submission: 2 December 1988

Keywords: Improved Graphical Text Model, Reference Model 
2 December 1988

US Contribution to the Improved Graphical Text Model Sludy

This is a US contribution to the first meeting of the SC24 Sludy Group on an Improved Graphical Text Model. This contribution is divided into several parts. These are:

1) Interpretations and Clarifications of the Terms of Reference (SC24 N172).

2) Goals for the Improved Graphical Text Model.

3) Requirements for an Improved Graphical Text Model.

4) Supporting material.

5) Identified issues.

Several annexes provide input documents that may be difficult to obtain otherwise. These are:

Annex 1. SC18WG1 N616 User Requirements for TCSS (DSSSL) and TPM (SPDL)

Annex 2. ISO DIS 9541, Parts 1-6, Font and character information interchange, 6 June 1988.

Annex 3. ISO DIS 10036, Procedures for registration of glyph and glyph collection identifiers

Annex 4. Examples of "registered" glyphs and commercial "fonts"

Annex 5. Xerox Interpress Electronic Printing Slandard, Version 3.0, Xerox Corporation, Stanford, CT, December 1985.

Annex 6. SC18/WG8 N715 Standard Page Description Language, Working Draft 4, December 1988. 
We interpret the virst paragraph of N172 this way:

Conduct a study to develop an Improved Graphical Text Model that will meet the graphical text requirements of a wide range of applications including, but not limited to:

- Office document creation, printing and exchange;

- The creation, printing and exchange of published documents;

- Technical drawing and illustration creation, printing and exchange;

- Graphics arts and presentation graphics; and

- Presentation entities within product data.

Furthermore, this study should consider the requirements for interworking between implementations of graphics standards and standards in other areas. As far as text is concerned, these other areas include, but are not limited to:

- Office and publishing systems,

- External representation of product definition data, and

- Open systems.

We suggest that the list of documents given in $\mathrm{N} 172$ be clarified as follows:

1) In the area of current computer graphics practice, the following document describing the "Hershey Fonts" should be considered:

- Hershey, Alan, A Contribution to Computer Typesetting Techniques, NBS Special Publication 424, April 1976.

2) In the area of related SC18 work, the material in Annexes 2, 3, 4 and 6 should be considered, as well as the ODA/ODIF Draft international Standard (DIS 8613), especially Part 6, Character Content Architecture.

3) In the area of available descriptions of commercial systems, the material in Annex 5 on the Xerox Interpress "system integration standard" and the following published (and widely available) documents should be considered:

- Adobe Systems Incorporated, PostScript Language Reference Manual, Addison-Wesley Publishing Co, Reading, MA, July 1985.

- Adobe Systems Incorporated, PostScript Language Reference Tutorial and Cookbook, Addison-Wesley Publishing Company, Reading MA, December 1985.

- Harrington, Steven J. and Robert R. Buckley, Interpress; The Source Book, Brady, New York, NY, 1988.

- Knuth, Donald E., Computers \& Typesetting, Volumes A-E, Addison Wesley, Reading MA, 1986 
- Karow, Peter, Digital Fomats for Typefaces, URW Verlag, Germany, 1987.

The SC18 user requirements documents in SC24/WG1 N7 are out of date. The updated versions in Annex 1 should be substituted.

The last paragraph of $\mathrm{N} 172$ discusses schedules. The US notes that only one meeting of this study group is listed in the resolutions of the last SC24 plenary, while the terms of reference calls for 3-4 meetings. To accomplish the work assigned to this study group the US believes that a total of 3 meetings is needed. The additional two meetings might be scheduled as follows:

- a meeting in conjunction with the April 1989 meetings of the Product Data Geometry and CGM extensions study groups; or

- a meeting in late July 1989 in conjunction with Reference Model Rapporteur group or early August 1989 in conjunction with the New API study group.

The US interprets the dates given in N172 for output document availability as requiring that the output documents produced by the study group be circulated to SC24 for review prior to the October 1989 SC24 meetings.

2. Goals for the Improved Graphical Text Model.

1) The model should support the identification and specification of important attributes of fonts, characters and text for the purposes of:

a) font selection and substitution, and

b) text rendering accuracy,

as further described in Clause 4.

2) Fallback guidelines for font selection should be possible.

3) The model should accomodate all Information in DIS 9541. Individual standards based on the model may adopt only appropriate parts of DIS 9541.

4) The model should have as much compatibility as possible with the existing text model used in current computer graphics standards without compromising DIS 9541 compatibility.

5) The model should distinguish between different types of attributes. At least three categories appear useful:

a) font attributes,

b) character display attributes, and

c) text string attributes.

6) Clients of the new model must have a way to determine the extent of text objects at the time that such objects are defined.

7) The model must be available for the next generation of standards. 
8) The model must be available for use in CGM Addendum 3.

9) A description of the model at an appropriate level of abstraction should be merged with the SC24 Reference Model work.

10) The model should support the development of standards for all uses of computer graphics. Such uses include graphics arts, publishing, and pre-press systems, as well as traditional business graphics, CADiCAM, and other scientific, technical, and mathematical applications.

11) There should be a single unified model for our entire family of SC24 standards. The model should also support the needs of all application areas that incorporate computer graphics. Graphical text within individual standards should be based on, but need not include all of, the model.

\section{Requirements for an Improved Graphical Text Model.}

Future API and metafile standards have the following requirements which the Improved Text Model must support:

1) It should be possible to have text objects whose text extent is workstation independent. (See subclause 4.2 for additional delails.)

2) It should be possible to determine the extent of a text object at the time it is defined.

3) Altribute changes within text objects should be allowed. For example, it should be possible to underline part of a text string. (See clause 4.1 for additional details.)

4) The model should allow exact font selection by standard (registered) font names.

5) The model should support additional attributes and characteristics, including:

a) scoring (e.g., underline, overstrike);

b) kerning control:

c weight (e.g., bold, medium, light);

d) posiure (e.g., italic);

e) subscripting/superscripting:

f) typeface design classification (e.g.. serif, sans-serif, Latin);

g) Iont family (e.g., Times, Garamond, Helvetica); and

h) others, the need for which may be determined in the future.

6) The model should allow the construction of complex compound objects. such as those required for mathematical equations.

7) The model should allow shielding/clipping to text images

8) The model should support 3D text and fonts.

9) The model should support multi-line text (for example, by defining the interaction of control characters with the text model.)

10) The model should allow explicit control over width as well as height of text. (See clause 4 for additional details.) 
11) The model should allow standards to provide successive levels of complexity in their text models. These are needed to enable simple things to be done pasily while giving advanced applications access 10 more powerful features. The current single-level model is too complex-for some and too simple for others.

12) The model should allow the layout of text along arbitrary paths.

13) The model should allow automatic font substitution.

14) The model should allow application-definable glyphs.

15) The model should allow access to font metric information:

a) average or global metrics, and

b) metrics for each character.

16) The model should allow the specification and application of user-defined transformations at various points in the transformation of text and characters.

\section{Supporting material.}

\subsection{Altribute changes within text objects}

It should be possible to construct a single text object that consists of parts with different attributes. In existing API standards this can only be accomplished by interspersing different output primitives, such as Text and Append Text, with attribute change elements. This makes it difficult to edit "compound text objects" and to identify and control the impacts of changes to edited structure elements. It may be appropriate to provide this functionality within the context of a more comprehensive object definition facility. (See subclause 4.5 for further information.)

\subsection{Workstation independent extent for text primitives}

The association of font indices to fonts and the realization of fonts are workstation dependent. Unfortunately, the extent of text primitives must be known for some operations performed above the Workstation Stage of the Reference Model. One example is the PHIGS modelling clip which cannot be properly performed on text primitives today since their extents are not available at this stage of processing. In metafile standards, blind interchange of quality text requires that generators be able to determine text extent and rely upon it being interpreter independent.

\subsection{Specifying the relative importance of attributes}

Some applications attach more importance to some text attributes than to others. There are at least iwo reasor.s for emphasizing some attributes over others:

- indicating font selection and substitution criteria, and

- specifying control over the accuracy of the rendering. 
Current standards provide some support for the second of these goals through use of the TEXT_PRECISION attribute. However, no support is provided for font attribute specification which would assist in font substitution or font selection by off-line or downstream text manipulation and/or generation services which may need to emulate the requested textual effect with the most closely matching available facilities.

The following proposals for accomplishing these goals are provided to initiate discussion:

\subsubsection{Font Attributes}

A function should be provided which associates font names and altributes with the font Indices used during font selection. This is analogous to specifying colours with colour selection indices. The function could also be used to download fonts or otherwise make them accessible. One way this facility could operate would be to specify a font name in a font lable as the CGM now does. The font name (Times Roman. New Century Schoolbook, etc.) itself implicitly define a set of font attributes which could be used as substitution criteria if the requested font name is not available. Such a font table would be workstation and device independent since it only depends on information about fonts whose characteristics are independently known.

Automatic font substitution has implications for both font resources and the font selection process:

- adequate descriptive information in the font resource: and

- mechanisms to allow applications to specify allowable variations on a font request or to specify requests with varying degrees of precision.

For example an application program that is attempting to do automatic font substitution must have access to enough information from font resources to enable it to determine the characteristics of available fonts and compute appropriate "best-approximations." Such approximation algorithms will vary from application to application.

Furthermore, application programs and metafiles need ways to explain the user's intent and desires where font substitution might be performed. For example, a user may desire only a specific named font (e.g., ITC Bookman), may be willing to settle for "similiar" fonts when the requested one is not available (e.g., use Adobe System's version of Allied Corporation's Palatino if available; if not, substitute Adobe's version of ITC Times-Roman; if that isn't available, the use any modern serif font; if there are no serif fonts available, then use any modern font: otherwise...) The depth of the substitution list should not be restricted by the model.

\subsubsection{Rendering Accuracy}

The current Text Precision attribute was introduced so an application could provide guidance to the graphics system on the trade-off between accurate rendering of text and efficient generation of text. It is appropriate to provide such guidance to the system. However, the current attribute is inadequate for modern graphics systems since it does not allow the application to indicate the relative importance of various text attributes in achieving its desired effect. 
One way of providing additional control over rendering accuracy might be to introduce a technique analogous to that used by the PHIGS Element Search function. Thus, a special type of name set could define an association between text attributes and names. An application could then provide one such name sels which has members whose associated text attributes are to be accurately rendered. Members not specified indicate text attributes whose values the system is allowed to modify as necessary for efficiency or to insure text fits within the extent of the text object.

\subsection{Text-related terminology}

\subsubsection{Definitions from DIS 9541}

Text-related terminology is evolving rapidly. Some traditional names, such as character, have been found to be too easily misunderstood and are being replaced by more precise terms, such as glyph. Some terminology is motivated by administrative considerations, such as the need to develop a clear separation between the traditional "codes and character sets" work of SC2 and the "font" work of SC18. Many of the definitions in the DIS text of DIS 9541 (Annex 2) were reworked at a Special Working Croup meeting held in London in September 1988 to harmonize the treatment of fonts among SC2, SC18, SC21, and SC24. The latest definitions are:

font: A collection of images having the same basic design, e.g. Bookman Italic.

font family: A set of fonts of common design, e.g. Bookman.

glyph collection: An identified set of glyphs.

glyph: An abstract graphical symbol independent of any actual image.

glyph image: The set of information defining the image of a glyph in a particular font resource.

glyph shape: The set of information in a glyph representation used for defining the shape.

glyph metrics: The set of information in a glyph representation used for defining the dimensions and positioning of the glyph shape.

font resource: A collection of glyph representations together with descriptive information and font metrics which are relevant to the collection as a whole.

score: A line drawn through a glyph shape parallel to the baseline [over, under, or through the shape.] 


\subsubsection{Deflnitions from ISO 2022}

The following definitions are extracted from ISI 2022:

bit combination: An ordered set of bits that represents a character or is used as part of the representation of the character.

character: A member of a set of elements used for the organization, control or representation of data.

coded character set; code: A set of unambiguous rules that establishes a character set and the one-to-one relationship between the characters of the set and their bit combinations.

\subsubsection{Concerns}

The definitions in subclauses 4.4 .1 and 4.4 .2 are not well reconciled. One goal of the study group should be to do such a reconciliation.

\subsection{Relationship of Text to Other Graphical Primitives}

The following model of graphical primitives explains the relationship of text to other graphical objects.

\section{Dimensienality Category Atributes Examoles}

\begin{tabular}{|c|c|c|c|}
\hline 1 & Linear & Polyline & $\begin{array}{l}\text { polyline, arc, ellipse, splines, } \\
\text { compound lines }\end{array}$ \\
\hline 2 & Area & $\begin{array}{l}\text { Interior } \\
\text { Edge }\end{array}$ & $\begin{array}{l}\text { filled polygons, filled circles, } \\
\text { cell arrays, spline surfaces, } \\
\text { compound areas (such as triangle } \\
\text { strips and quadrilateral meshes) }\end{array}$ \\
\hline 3 & Volumetric & $? ?$ & $\begin{array}{l}\text { cylinder, sphere, block, CSG, } \\
\text { compound volumes }\end{array}$ \\
\hline & High-Order & $? ?$ & $\begin{array}{l}\text { blinking primitives in which time } \\
\text { is the } 4 \text { th dimension }\end{array}$ \\
\hline & Composite & per prim. & $\begin{array}{l}\text { markers, text, annotation text, } \\
\text { symbols }\end{array}$ \\
\hline
\end{tabular}

In this context, compound primitives are primitives which are composed of multiple instances from the same category. For example, a compound line could be defined in terms of polylines and arc segments with the linetype pattern being applied continuously along the entire compound primitive. Similarly, compound areas are enclosed areas whose boundaries are defined by instances of linear primitives. This would provide, for example, easy definition of boxes with rounded corners.

Composite primitives are primitives comprised of examples from any of the categories. For example, the shape information of text glyphs could be defined in terms of line, enclosed area, or compound volume information depending on the needs of the particular font.

Many typefaces today are defined as compound areas whose interiors are then filled. Stroke fonts are defined in terms of polylines. Similarly, bit-map fonts are defined in terms of cell arrays. 
It should be noted that the current Cell_Array primitive is constrained 10 have all cells rendered. More powerful capabilities could be provided by introducing cell array attributes which would specify an "auxiliary" colour and a flag for indicating whether "auxiliary colour"-ed cells are to be rendered or the background is to show through. A cell array can also be considered as a compound primitive composed of a grid of cells with each cell being a filled polygon.

Composite primitives can be defined in terms of primitives from any category. Thus, symbols could be defined hierarchically and user-defined or system-defined glyphs could reference other glyphs to produce glyphs for logos or mathematical expressions.

Graphical transformations apply to all primitives uniformly. Lighting and shading information can be applied using standard rendering techniques. Since composite primitives are composed of other primitives they need not be excluded from realistic rendering operations. 
Issue: T4

Should font altributes such as italics be part of a font name, a font attribute, or both?

Hislory:

12.02-1988, Raised by U.S.

Keywords:

Improved Graphical Text Model

Alternatives:

1) part of a font name.

2) a font attribute.

3) both.

Arguments:

a) Pro1: All necessary resources known before interpretation.

b) Pro1: Consistent with typographic usage.

c) Pro 2,3: All possible combinations in a font name would soon become unwieldy.

d) Con 3: May result in ambiguity if different values are specified in the name and the attributes.

Issue T5:

Should text facilities allow access to attribute groups appropriate History:

to their shape defining primitives?

Keywords:

12-02-1988, Raised by U.S.

History:

Improved Graphical Text Model, glyph shape

12.02 .88

Alternatives:

1. yes

2. no

Arguments:

a) Pro 1: The possible special effects in displaying text would be enhanced.

b) Pro 2: Text usage is inherently different from that of other primitives. 

Attachment 3:

Minutes of the Munich CGM Extension Meeting 


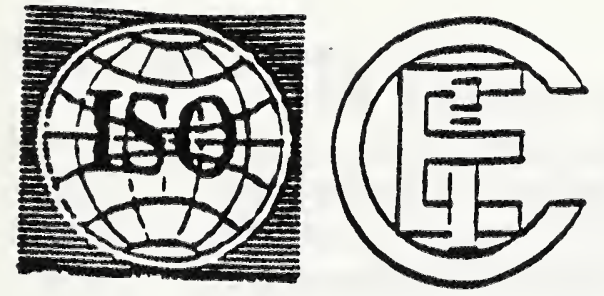

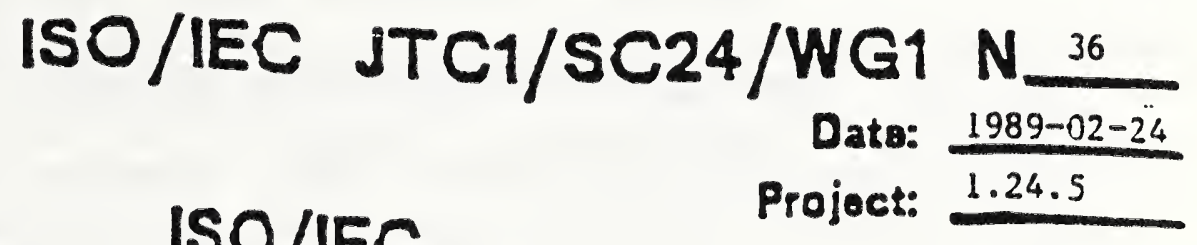

ISO/IEC

International Organization for Standardization International Electrotechnical Commission

\section{ISO/IEC JTC1/SC24/WG1 \\ Computer Graphics Architecture}

Secretariat: National Computer Graphics Associatior

Titla: Minutes of the Study Period Meeting for

Extensions to CGM Static PIcture Capture Capability

(Munich, Jan. 1989)

Sourca:

Meeting Secretary (A. Mumford)

Status:

- WGI Output Document

- Mambar Body Position

- Expart Opinion

$x$ Rapporteur Group Output

Typa:

I AT Immediate Output Document

A2 Delayed Output Document

- B National / Expert Contribution

- C Recant Liaison Document

Distribution Typa:

× Through WGi Socratariat

- Diroct to Membars

- Through SC 24 Secrotariat

Date of Submiasion:

Kaywords:

Minutes, CGM 


\section{ISO/IEC JTC1/SC24 Study Period Meeting for: Extensions to CGM Static Picture Capture Capability \\ 18-20 January 1989 - Munich, F.R. Germany \\ Minutes of the Meeting}

\section{Liaison Meeting}

The meeting began with a joint meeting between the CGM group and the other groups who were meeting during the same week. These groups were the Product Data Geomery srudy group and the improved Text Model study group. The minutes of that meeting are appendec to these minutes.

\section{Participants}

The participants in the CGM meeting were:

Germany: Moeller, Brandenburg (till 19th pm), Zapomuel (part of the meeting), Schuur (till 19th pm)

UR: Mumford, Francis, Thomas (part of the meeting)

USA: Bono (tiil 19th pm), Laris, Stoll, McConnell (par of the meeting)

Apologies were received from the Rapporteur, Lofton Henderson. Peter Bono chaired the meeting until Thursday pm.

\section{Aims of the Meeting}

These were to follow the nex SC24 guidelines (SC24/N171) and to produce a draft requirements document and a drat new work item for consideration by WG1. The Requirements Document and the draft New Work Item will go to the Reference Model meexing in Paris the week after this meeting and to WGI 28th Feb in Darmstadt. The new work requirements of SC24 require an SC24 ballot prior to the ITC1 ballot.

Relevant Documents

SC24N9 - Requirements Study

SC24N15 - Proposal for a CGM Addendum 3

SC24N52 - An Initial Draft of Addendum 3 produced by ANSI

TC188/SC4/N284 - STEP

SC18/WGR/N715 (Rev) - SPDL

SC24N177 - SC24 Proposed Reference Model

There were no official inputs to the meeting. 


\section{The Way Forward}

It is hard to define the precise nat re of the work as it is so closely tied in with the other st 'dy groups. They need to report before the final requirements can be drawn up. There was concern that this may delay the work which is needed in the market place. A revision of the current Addendum 3 will not be circulated with the NWI as it may confuse and pre-empt recommendations of the other study groups. This would not prevent work on the document being carried out by a national body taking account of these discussions in Munich. the document when it was eventually produced taking account of all study group reports would benefit by review at this early stage.

It was agreed that the purpose of the meeting was not primarily to develop the Addendum 3 work based on previous drafts. The aims were far wider and the purpose to recognise requirements rather than to define precisely how these might be met.

\section{Timescales}

When drawing up the timescales account was taken of the fact that some countries had difficulty in participating in so miny meetings. It was agreed that a tight schedule which took account of other meetings and held them at the same time was preferable to holding separate meetings which required the same experts to attend. Particular attention was paid to the CGMJGKS addenda editing meeting in June/July in Hawaii and the SC24 meeting in October in Brazil. The following timetable is proposed:

28 Feb 89 SC24/WGI approve output from Munich meeting

3 March SC24 approve NWI Bailot

15 March NWI ballot starts

15 June NWI Ballot closes

3 July Meeting in Hawaii to revise NWI - faxed to SC24

5 July SC24 forwards ballot to JTC1

15 July JTCI ballot starts

15 Oct JTCI Ballot ends

16-29 Oct Working Draft prepared at SC24 meeting

Apri 90 DP/PDAD text prepared

Sept 90 DIS/DAD tert prepared

April 91 IS teat prepared

This is a very tight schedule. It was considered that it was necessary at least for the first few stages to ensure improved participation.

Liaison is needed between SC24 and SC18 at their meening in Munich and at the STEP meeting in San Antonio. We need an SC24 rep not necessarily a member body representative.

Action for the Rapporteur to request liaison at these meetings and to ensure participation.

Discussion on the Requirements

The Tucson meeting discussed the relationship with SC18. The recommendations (SC24/N186) include the need for SPDL to transiate a CGM into SPDL in a standard way. 
ODA allows this and includes CGM in its specification. Areas of overlap need to be solved in a common way.

There was agreement that extensions to the CGM might include publishing, engineering drawing, business graphics as included in doc. N9. Cartographic requirements are also needed. When do we know when to stop adding functionality? The requirements document must state this.

It was agreed that Addenctum 3 (or whatever it becomes) should not be 3D and that it should be built on CGM plus Addendum 1. Then possibly extend to 3D for added capabilities.

It was agreed that a closed list of elements might be better for getting a standard produced than less clearly defined requirements. The precise list should be agreed when the study periods come to an end (Oclober 89) It is hard though with the work going on in parallel within SC24 and outside in other ISO groups. The STEP work is in early stages (DP) of standardisation and their timescale is less agressive.

The discussions as to the precise requirements were based on a consideration of N15. 6 areas of extension were recognised:

1. Advanced $2 D$ requirements

curves - note STEP line extension might be of interest

fine control over line appearance

composite line primitive

user defined line types, harch styles, markers - also symbols required with the same definition techniques as markers - and glyphs

additional standardized hatch styles

arbitrary text path

are filling methods sufficient? there is a need to make up styles from

the other primitives - note CGI Bitmap fill too but this is not device independent

general linear transformalion - need for $3 * 3$ matrices

2. Text and Font Model

take the requirements from the study group on text

3. Arbitrary Boundaries

There was concern as to the number of start-end boundary sequences there are in the doc N52 (plus closed figures in Addl and CGI). It would be better to have a general path element which would then have its action applied to it. 


\section{Colour Mc jels}

This is definitely a requirement and close study is needed of the ODA colour Addendum. Colour interpolation is also needed.

\section{Image .}

SC2/WG8 have work in the area of compression techniques. Their documents are at DIS level and should be used. Can cell array be improved by making it more compact in its encoding

6. Symbols

There is a need for defining symbols and also for extemal referencing - this is a general requirement for other areas e.g. fonts.

Another requirement might be for directories of pictures to be stored.

(Alignment (N15) left out as nobody could explain it)

There was some concem that a third addendum to CGM might not be the best way to progress the document. Addenda are confusing. This would also be difficult if there is another colour model as RGB is described in many places. A revision would be better but this would have many implications e.g. doing 3D fully.

It was agreed that the extensions work was to address storage capabilities at the same level of the reference model as CGM and Add 1.

\section{STEP/CGM Reference Models}

The group discussed the diagrams presented in $\mathrm{N} 257$ The discussion was led by $\mathrm{Mr}$ Zapomuel. Presentation enuries are on the border of the CAD system and the graphics package.

Some comments were made on the specifics of the diagram. CGM and CGI are not necessarily at the right place relative to the new CGI model. the graphics package can ailso bc wide or narrow depending on the implementation (though conceptually some of the layers may still exist). It would be useful to add the PHIGS archive and GKSM in. It was agreed that this was one example of how things fitted together rather than being a definitive statement.

The bell curves on the second diagram were felt to be a useful representation of the position of the various standards. McComell to tale this diagram to the Reference Model meeting. STEP should end where CGM starts. STEP and CGM should replace IGES giving no overiap between the standards. There needs to be a definition of the differences between a drawing and a document. 


\section{Reference Model}

The Reference Model draft (N177) was presented by John McConnell. The group discusscd the model in relation to the CGM and GRSM and the comments are to be fed back to the Reference Model group.

The CGM is a single workstation withe coordinates stored in virtual device coordinates. The Workstation level is thus the most likely point for the CGM to lie. Elements such as pixel array are probably lower and this is likely 10 be a point of concem in some of the comments on the Add I ballot. If we are to say that the CGM extensions are at the same level as CGM can raster ops be added (as currenily proposed).

There was some concem that the model could have multiple coordinate systems at the same level. This makes it hardes to place CGM. Could CGM be a list of elements which range across a mumber of levels of the metafile with the Metafile Category being used to identify a particular sor of metarile and thus where it lay in the model. This is not in line with the idea that CGM is well defined in the model.

It was noted that the CGM extensions work is a part of the first generation of standards and thus this model does not necessarily apply. Also there will not necessarily be standards for all levels.

\section{Discussion on the Initial Draft (N52)}

The document N52 which was produced by ANSI some time ago was discussed with the expers making poinis which can be fed into the next dratt. The comments are appended to the mimutes.

\section{Output from the Meeting}

1 These minutes

Action: Mumford to draft and send to Bono for circulation

2 Draft Requirements Document

Action: Group to draft, McComnell to take to Ref Model meetings and then to arrange circulation in WGI via Bono

3 Draft New Work Item

Acrion: Group to draft, McConnell to take to Ref Model meetings and then to arrange eirculation in WGI via Bono

4 Letters from the Rapporteur to Reference Model and Requirements groups in WG1 and to WGI convenor requesting action on the documents.

Action: Bono to draft and send to Henderson 


\section{A Jpendix 1}

\section{Comments on The Initial Draft for Addendum 3 (SC24/N52)}

This was a very brief discussion but the following points emerged:

1. Advanced 2D Requirements

a) curves

STEP Geometry has the following 2D primitives (Presentation also needs to be checked) conic: circle, ellipse, hyperbola, parabola

bounded curve: polyline, B-spiine, trimmed curve, composite curve

SPDL must be checked to see if the representation of the curves defined in N 52 is the most efficient for SPDL.

b) line appearance

STEP parameterises the end point of the line which can be user definable (4.12.5 in STEP preseniation)

STEP also has rounded asymmerric line join. These considerations also apply to edges.

c) composite line primitive

There was a general agreement that the same method should be used for shielding and clipping. Too many begin-end pairs appear in N52 Should this also be adopted for closed figure? There are difference here though. Need to look as SPDL paths. There may be conflict with CGM and Addl though.

d) user defined line styles etc.

There was a feeling that the needs of cartography had not been addressed and that they shoukl be.

e) additional hatch styles

There is a need for styles to be derined from the other primitives. We need a wider capability for filling of areas than just cross hatching. How should this be addressed - what is a hatch?

f) arbitrary text path

the begin-end path comments addressed above apply here too.

8) filling 
PostScript has the non-zero winding rule as well as the odd/even filling. (pg 71 red PostScript book). Interpolated filling (going from one colour to another) also seems important.

h) general linear transformations

These are not in N52 but would be useful.

2. Improved Text and Font

Strong feeling that the work of SC18 and the work of the study group on the improved lext model must be the main driving force for the elements in this section.

3. Clipping and Shielding

What does text shielding apply to - the character box or the shape? This needs to be addressed in relation to the discussions on glyph definition in the improved text study group.

\section{Colour Models}

There is nothing in N52. This needs to be addressed but it will be one of the hardest things to add in if the text is to be an addendum. RGB is mentioned very many times in the CGM lext. The ODA colour model and PHIGS should be used as base documents for the elements.

\section{Imaging}

The reference model makes this a problem. How can the CGM+Add3 be at the same place in the reference model if these more device dependent elements are added in? It was recommended that the work on picture coding standards in SC2 should be the basis for any definition. Do we need Pel Array Clip Rectangle? Does general clipping apply to raster? Arbitrary dipping of raster might be useful too.

6. Symbols

Has Add 1 dealt with this? If not, why not? 
Attachment 4:

Addendum 3 New Fork Item Proposal

Addendum 3 Requirements Document 



\section{CGM ADDENDUM 3 NEW WORK IIEM PROPOSAL}

\section{Scope}

This work comprises a set of elements which will extend the capabilities of the CGM (IS 8632) and CGM Addendum 1 (ISO 8632/DAD.1) to meet additional user requirements.

The following list of capabilities will be addressed by this work

1) Advanced 2D graphics, to include:

- curves

- fine control of line appearance

- composite line mimitives

- user defined line types, harch styles, and marker types

- additional standaritized hatch styles

- arbitrary text pach

- Elling mechanisms

- general linear transfomations

2) Improved text and font support

3) Arbitrary boundaries for clipping and shieiding

4) Additional color models beyond RGB

5) Additional raster graphics (scanned image) capabilities

๑) Symbois: external reference to "standard" libraries of named symbols

The precise list of elements to be inciuded in this group will ake account of the work of the SC24 study groups in the following areas: Improved Graphical Text Model, Product Data Geometry, new API for graphics, PHIGS BR, Reference Model for Computer Graphics, and also the GKS Maintenance work

\section{Purpose and Justification}

The purpose of this work is to extend the CGM and CGM Addendum 1 to fulfill additional 2D picrure storage and retrieval requirements. CGM users have found that in some application areas the present standard provides a general framework that is suitable but lacks some functionality required by these applications. These areas include engineering drawing, the preparation of graphic arts qualiry presentation materials, carrography, and technical publishing.

SC24 has recognized the need to serve these application areas and to meer the requirements which go beyond those currently specified in the standards developed, and being developed, within SC24. The precise requirements will be the result of the deliberations of the smdy groups set up by SC24 to consider an Inproved Graphical Text Model, Product Data Geometry, and a new Application Programming Interface for Graphics. It is considered that meeting these requirements is essential if the CGM is to continue to be used in the areas recognized above.

It is essential that this work uses some of the solutions adopted within related ISO Standards activities. for example font definition standards, colour models, and product dara exchange standards. 
Co-operation and Liaison

Work will progress within ISO/EC JTC1/SC24 in liaison with:

ISO/IEC JTC1/SC18

ISO TC184/SC4

ISO/IEC JTC1/SC2

Relevant Documents to be Considered

CGM, IS 8632 (Parts 1-4)

CGM Addendum 1, IS 8632/DAD-1 (Parts 1-4)

Font and Character Information Exchange, ISO DIS 9541 (Parts 1-6)

ODA, ISO DIS 8613 Colour Addendum

PHIGS BR

Reference Model for Computer Graphics

STEP, ISO TC184/SC4/N284

Program of Work

The following schedule is proposed for this work:

Oct 1989 - NWI approved, WD prepared

April 1990 - DP or PDAD text prepared

Sept. 1990 - DIS or DAD text prepared

April 1991 - final text prepared

Assignment of Work

SC24 requires that this work item, if approved, be assigned to SC24/WG3. 


\subsection{Introduction}

It has been recogmized at at the curreat CGM Standard needs to be extended in arder to effe jively fuifill the picure storage and transfer requirements of engineering drawings, graphics arts and technical publishing. The purpose of this document is to identify the requirements for extension explicitly, as specified by the procedures stipulated in ISO/IEC JTC1 SC24 N171.

\subsection{Applications}

\subsection{Application Areas With Further Requirements:}

\section{a) Engineering Drawings}

The CGM is courently being used in engineering applications to store and exchange picurre information. This ares of use has found the CGM lacking in functionality which, if present, would increase the efficiency of storage Examples of required elements inciude curve definitions, such as B-spline and conies, and improved text capabilities. In other areas more control is required. Examples of this include control of line appearance such as line ends. There are also requirements for specifying and referencing symbois.

\section{b) Graphies Arts}

For graphic arts quality a higher degree of control over the rendering of graphical and text objects is required Line atributes are required to control the rendering of line endings and line joins and user-derined line types are also required. There is a requiremeat to be able to print text along an arbitrary path and to specify fonts and text auributes in a more precise manner. Further colour models and raster encodings are also required.

\section{c) Technical Publishing and Illustrations}

One of the main intentions of the CGM Standard is to support the requirements of this application area. Though the CGM has been used successfully in the technical publishing and illustration environment, it is felt that some of the inherent limitations in the CGM Standard have curtailed its acceptance in the markepiace. Several enhancements to the CGM have been identified to address these limimations, inciuding but not limited to: line appearance control, arbitrary text path, enhanced text capabilities, and additional coilow models.

\section{Studies of Applicarion Requirements}

\section{a) Product Data Geometry Stridy Group Report - PDG SG}

The purpose of this stody group is to look at the relationships between Product Data Geometry (PDG) and Computer Graphies; its memberstip should be drawn from experts from the Computer Graphics Standards and Product Dam Geometry Standards commmnities. In discussing these relarionships at the first meeting in Mumich, it was found that there exists some overiap between the stared goals of the PDG Standards and the CGM. The SG concluded that in certain areas the CGM, if extended, conld be urilized to fulfill those goals. Some of the requirements for extension included complex curves, additional user-defineable atributes, additional font/text functionality, and symbol libraries.

\section{b) Text Study Group Report - Text SG}

Given that SC24 has recognized that the current text model needs review, this stady group has been chartered to look at what enhancements are necessary and/or desired for the existing graphical texi models. The main conclusion of this SG at the Munich meeting was that the Font Standard work tating place in SC18/WG8 should be the primary souree of technical input for improving the graphical texs model. The SG also uncovered some additional requirements for text in studying the 
STEP seandard. Some of the enhancements identified for the CGM included font definition and referencing. font substimion, and rendering.

\section{c) CGM in the Real World Workshop - CRWW}

This Workshop, involving implementors of the CGM, mex in September of 1987 to discuss the successes and difficulties encoumered when implementing the CGM Standard. At this workshop, several common problems and shortcomings of the CGM were identified. Some of these included, but were not limited to: the inadequacy of the text model, the lack of advanced 2-D graphics primitives, and the need for additional colour models.

\section{d) User Requirements Stady of Publishing and Technical Drawings - WG1 N9}

This report was prodnced under the auspices of the U.S. DoD Computer-Aided Acquisition Logistics Suppor (CALS) Office CALS is a major initiative imended to antomate the production, exchange and publishing of product support information. Product support information in CALS includes product data, raster graphics, 2-D technical drawings and text. This stody (WG1 N9) specifically addresses the extension requirements for the CGM to support the technical illusuration exchange and storage needs for CALS. Several needed enhancements are identified, including bezier corves, line cap and join, enhanced text, shielding, interpolated fill, and additional colour models.

e) Input into the GKS Review - Cartographic Requirements (CR)

This document is a requirements statement put together by experts from the cartographic industry regarding the enhancements needed to GKS to support cartographic applications. These enhancements are also required for the CGM, and include: geometrically specified patterns, artiurary clipping regions, and external symbol libraries.

\subsection{Required Capabilities}

The following table provides a list of the feanures that were identified as requirements in the studies mentioned in Section 2 The feanres are listed along with the smdy from which they originared:

-FEATURE-

Advanced 2-D graphics:

- curves

- line appearance control

- composite line primitives

- user-defined line,hatch,marker types

- addirional standardized hatch styles

- arbiurary text parh

- geomerrically specified paterns

- general uransformations

Enhanced text and font capabiliries:

- fontglyph definition

- font referencing and substiution

- rendering

- additional text auributes

Arbitrary boundaries:

- clipping

- shielding

Colour models:

- CIE CMYB, named colours, etc.

- interpolatod fill
-REQUIREMENT SOURCE-

PDG SG, CRWW, WG1 N9, Text SG

PDG SG, CRWW, WG1 N9

WG1 N9, CRWW

WG1 N9, PDG SG, CRWW

WG1 N9, PDG SG

PDG SG, Text SG, WG1 N9

PDG SG, CR

PDG SG

Text SG, PDG SG, CRWW WGI N9

see above

see above

see above

WG1 N9, CR, CRWW

PDG SG, WG1 N9

WG1 N9, CRWW

WG1 N9 
Addirional raster graphics:

- coding/compression techniques

WG1 N9

Symbols:

- external lib.

- use vefined internal lib.

WGI N9, PDG SG, CR

see above

\subsection{Examples}

\subsection{Advanced 2D Graphics}

The CGM in the Real Worid Workshop examined the issue of advanced 2D graphies and concluded that the CGM lacked capabilities to effectively meet some advanced user needs. For example, for engineering drawings it is difficult, if not impossible, to effectively represent some higher-level constructs in the CGM, such as splines and curves. Though such constructs can be simulated with simpler primitives in the CGM, it is frequently impossible to maintain accuracy and visual continuity and still retain device independence. $A$ list of additional functional requirements for advanced 20 graphics follows, with examples of the realization requirements:

\section{a) Curves}

Curves include the general class of curved line elements that are more complex than the existing circular and elliptical arc elements, such as:

- Bezier curves

- Rarional B-splines

- Parametric spline curves

- Conics, and conic ares

b) Fine Control of Line Appearance

This includes the additional line attributes of cap, mitre, and join.

c) Composite Line Primitive

This consists of a line composed of both straight and curved line segments.

d) User Defined Line Types, Harch Styles, and Marker Types

An example would be the ability to define a line type, such as the stitched, center, or phantom line types frequently used by engineering drawing applications.

e) Arbitrary Text Path

Text drawn along a composite line path.

f) Fuling Mechanisms

Interpolated Fill, which is a fill comprised of colours interpolated linearly between two reference colours.

g) Geseral Linear Transformations

$3 \times 3$ (and $2 \times 3$ ) transformation matrices to allow for affine and projective transformations

\subsection{Enhanced Text and Font Capabilities}

The enhanced text and font capabilities should accomodate most of the informarion in the ISO DIS 9541 Font and Character Information Interchange Standard. This standard defines a font resource architecture to support text generation, interchange and presentation. A font resource has to provide sufficient information to characterize and identify a font in order to allow font referencing and font 
substitwion. In the latter case, mechanisms to specify allowable variations on a font request or requests with varying degrees of precision should be provided.

Font and glyph defimition requires attributes in addition to those used in the cument computer saphics text model, such as typeface design specificarion, posture, and kerning control. However, to support feanures such as arbitrary text path, information cumently not included in ISO DIS 9541 would have to be provided.

Techniques used in modern graphics systems must be provided to guide the interpreter of a metafile as to the desired rendering accuracy to indicate the relative importance of various text atributes for achieving a desired effect.

\title{
5.0 Constraints
}

The work which is proposed to meet these requirements will be based on the CGM standard and CGM Addendum 1. It is intended that this extension will occupy the same point in the SC2A Reference Model as the original standard. This means that the exctension will produce a metafile suitable for the storage and retrieval of 2-D picture information, and will not address 3-D or dynamic capabilities. If is the goal of this work that it will be useable by related standards including the font standardization effort, ODA, and STEP.

Close working with the GRS Maintenance group is essential. Where this group defines functionality which is the same as that proposed for this extended metafile work, then the groups should work together to produce functionally identical specificarions and encodings. The progress of this work will be measured by its relationship to these other standards and their resulting compatibility.

The New Work Item proposal defines the areas of extension and notes the need to adopt the results of the study groups of SC24. If is strongly recommended that these functional requirements are umed into realization requirements as defined by SC24/N171 on acceptance of the NWI and as the working draft is being prepared. This means that if the proposed schedule is adhered to, then the general requirements will be trmed into a list of elements to be included in the new work at the SC24 meering in October 1989. This does limit this work to extend the CGM to include only those requirements which have been accepted at that time. This should ensure that progress can be monitored, problem areas identified early in the project, and these urgent needs for CGM users met in a timely fashion.

\subsection{Bibliography}

\author{
STEP - TC184/SC4N284 \\ IGES V3.0 \\ EDIF \\ PHIGS BR - JTC1/SC24/N224 \\ GKS Review - Cartographic Requirements \\ Font Standard - ISO/DIS 9541 \\ ODA - ISO 8613 \\ SPDL - JTCL/SC18/WG8/N715 \\ Liaison Staremear to SC18 Regarding SPDL - SC24N208 \\ Terms of Reference for Improved Text Model - SC24N172 \\ U.S. Contriburion on the Inproved Graphical Text Model \\ GKS - ISO 7942 \\ PostScript - Reference Mamual and Tutorial \\ Interpress - Introduction \\ Mociem Drafting Practices and Standards Manual \\ CAIS - MUL-STD-1840 \\ Line Conventions and Lettering - ANSI Y14.2M 1979 \\ Dimensioning and Tolerancing - ANSI Y14.5 \\ CGM in the Real World - Springer Verlag, 1988
}


Attachment 5 :

Final Report of CGM Extension study Period 

Final Report of CGM Extension Study Period

Lofton Henderson, Rapporteur

10 August 1989

\section{INTRODUCTION}

At its July 1988 meeting, ISO/EC JTC1/SC24 passed a number of resolutions establishing study groups and study periods on future computer graphics standards work within SC24. Resolution 9 established a study period to examine the need for further extensions to the Computer Graphics Metafile standard (CGM, ISO $8632 / 1987$ ). Further extensions had been proposed in order to meet the requirements of application areas such as engineering drawing, technical publishing; and graphic arts, and had commonly been referred to as "Addendum 3."

One set of extensions, Addendum 1, was already in an advanced state of processing. A second set, Addendum 2 (for 3D), was technically an active project but was without a document editor and was not progressing. Both of these projects were being processed under ISO rules for addenda, according to resolutions at the SC24 plenary in September 1986. These resolutions established the addenda projects without New Work Item review and ballot.

It was determined by SC24 that the proposed Addendum 3 would be progressed according to the new NVI procedures in N171. Under these procedures the need for such a project would be studied by a study period, a requirements document would be generated and reviewed by the requirements rapporteur group within SC24/WG1, the position of the proposed work in SC24 reference models would be reviewed by the reference models group within WG1, and finally an NWT would be generated and balloted.

Key technology areas of Addendum 3 include advanced graphical text capabilities and advanced geometric primitives. Because these technology areas are expected to be shared among several of the next generation of SC24 standards, study groups were established to examine each of the areas. The Addendum 3 study period was directed to coordinate closely with these two groups.

\section{SUMMARY OF RESULTS}

The Study Period concluded that the extensions referred to as "Addendum 3 " are needed and must be expedited if CGM is to continue to be an important standard.

The provisions of $\mathrm{N} 171$ were followed. A requirements document and reference model statement were produced and submitted to WG1. A New Work Item proposal was drafted and submitted to SC24 for a three month ballot. The ballot passed with no negatives, and a slightly revised NWI is currently being balloted in JTC1. A Working Draft of Addendum 3 has been produced and is currently undergoing a three month comment period in SC24. 


\section{HUSTORY}

\subsection{Munich Meeting}

The first meeting of the Study Period was held in Munich, F.R. Germany, 18-20 January 1989. It was held in parallel with the initial meetings of the Text Study Group and the Product Data Geometry Study Group. These two groups met for the first half of that week, and the CGM group met for the latter half. Many of the attendees participated in one or the other of the technology study groups and then the CGM meeting.

The goals of the meeting were to ascertain the need for CGM Addendum 3 and execute the first steps in the new NWI procedures as detailed in SC24/N171 produce a requirements document, a Reference Models statement, and a draft NWI, and forward these documents to the appropriate groups.

The CGM meeting was attended by:

Germany: Brandenburg, Moeller, Schuur, Zapomeul.

UK: Francis, Mumford, Thomas.

US: Bono, Laris, McConnell, Stoll.

The meeting was chaired by Bono in the absense of the rapporteur Henderson.

There were no official inputs to the meeting, but base documents referenced in the meeting call included some requirements studies and a draft NWT proposal.

The output of the meeting consisted of:

- Minutes (WGI/N36).

- Draft Requirements Document;

- Draft New Work Item proposal;

- Letters to the WG1 Reference Model RG and the Requirements RG requesting action on these documents;

The detailed results of the meeting are contained in these documents. The final versions of the NWI and Requirements Document are included as an attachment to this report. Significant points of the meeting are summarized here.

The dependence of the final output of this Study Period on the outputs of the Text and Product Data groups was recognized. Concern was expressed that this dependence could slow the work down considerably. It was agreed to progress the work as much as possible, but there would be need for looking at the final outputs of the two groups when they become available.

There was some discussion of how to progress the project - should it be an addendum? The general feeling is that it should not, because this would be too unwieldy. Decision on the exact method of processing will be deferred until a later date. 
A schedule was derived which would result in IS text in April 1991. This included a tight schedule to complete all of the required NWI processing before the SC24 meeting in October 1989. By the end of that meeting there should be a complete list of requirements and a closed list of elements.

The next and final scheduled meeting was set to be in Waikoloa, Hawaii, 26-28 June. If the schedue were kept this meeting would process the results of the SC24 ballot on the NWI proposal.

\subsection{Between Munich and Waikoloa}

The Draft NWI and Draft Requirements documents, with cover letters from the rapporteur, were sent to the Reference Model Rapporteur Group and the Requirements Rapporteur Group as per N171.

The Reference Model group responded informally. Because there are no formal liaison documents, that response will be reviewed here. The Reference Models RG pointed out that there could be some problems with asserting that the proposed CGM Addendum 3 occupies the same "level" in the reference model as CGM. This problem was seen during the processing of CGM Addendum 1, which at that time contained a low-level device dependent formulation of the Pixel Array element (it was removed from Add.1 for this reason).

The Reference Model RG felt that the same problem could arise in Add.3, particularly if care is not taken in formulating the additional imaging capabilities. They pointed out that if the realization of the proposed functions of Add.3 straddled stages or levels in the Reference Model, then CGM would have to make a choice: either CGM-plus-addenda is at a well defined stage in the pipeline and any functions to the contrary would be proscribed and removed; or that principle no longer pertains and CGM-plus-addenda would be viewed as a set of encoding techniques to be applied to objects at different stages in the pipeline.

The general consensus of the CGM Study Period and the WG3 CGM Rapporteur Group is that the CGM standard should remain as a static picture capture mechanism whose features can be placed at approximately the current CGM level in the pipeline. This is an issue that CGM must keep in mind while progressing any addenda. Neither CGM nor Reference Models have been able to precisely specify what criteria determine whether functions are at the same level or stage. This question is bound to be subject to some interpretation, and will likely always generate differing opinions. But at least there should be some clustering around a stage in the pipeline, and elements which create recognizable technical problems (such as the CGI PIXEL ARRAY that was originally included in Add.1) should be avoided.

The Requirements Rapporteur Group of WGI did not generate a formal response to the Draft NWI and Requirements documents either, apparently due to scheduling difficulties. There is in the WGI document register (WGI N41) a U.S. position paper. A couple of comments in this paper seem pertinent. In the area of constraints, the CGM requirements document does not adequately express: requirement for the availability of resources; requirement for timeliness (must be done by YYMM). These points must be kept in mind by the Metafile Rapporteur Group. 
Because there were no requirements for change from either of the WGl rapporteur groups, the same two documents that were produced by the CGM Study Period at Munich therefore went to the WGI plenary meeting in late February. Recommendation 3 of that meeting recommended that the SC24 Secretariat immediately conduct a three month ballot on the NWI so that the results could be processed at the final scheduled meeting of the Study Period, 26-28 June in Waikoloa, Hawaii.

The three month ballot was commenced as planned, and terminated on 15 June.

\subsection{Waikoloa Meeting}

The Waikoloa meeting was the last scheduled meeting of the CGM Extensions Study Period. Its purposes were to:

- process the results of the NWI ballot;

- revise, if necessary, the NWI and the Requirements Document;

- express these to the SC24 Secretariat, to be forwarded for a 3-month JTC1 ballot to close before Brazil.

- do technical work on the Working Draft.

The meeting was held in parallel with a meeting of ANSI X3H3. It was attended by:

Germany: Eckhard Moeller.

UKK: Alan Francis, Anne Mumford.

US: Bruce Garner, Lofton Henderson (Rapporteur), Mike Laris (provisional Document Editor), Lori Pearce, Harold Schechter.

Anne Mumford is succeeding Eckhard Moeller as rapporteur of the WG3 Metafile RG, which will be processing Addendum 3.

There were no formal inputs to the meeting. The U.S. produced and circulated to attendees a baseline document to serve as a starting point for a working draft. The SC24 Secretariat sent NWI ballot results by Fax. The results: 9 approve, 2 approve with comments, 0 disapprove, 0 abstain. Comments were received from:

U.K. - stressing importance of liaison and harmonization with closely related groups;

Japan - making suggestions that the NWI and Requirements Document be improved by providing more details.

The comments of the U.K. were thought to be addressed adequately in the current document. Some time was spent discussing the comments of Japan. The additional detail suggested was deemed to amount to a justification of the requirements that had been generated from various sources. While such more detailed information would in fact be of interest, it was thought to not be a necessary component of the documents required by $\mathrm{N} 171$. In any case the group concluded that it did not have 
the resources to develop the additional information, as that information would essentially have to come from the source of the requirement. As can be seen from the Requirements Document, those sources are diverse and were not generally present at the meeting.

Conser":ently, minor improvements to the documents were made and the rapporteur sent these on to the SC24 Secretariat for commencement of the SC24 ballot.

By the time of this meeting there had been no further activities in either the Text Study Group or Product Data Study Group, and so the NWI and Requirements Document still contain references to the work of those groups as the source for specific functional requirements.

There was a liaison meeting between the CGM group and a number of outside experts interested in STEP, PHIGS, PHIGS+, and reference models. The topic was metafile requirements that are derivable from STEP and PHIGS $(+)$. As far as Add.3 is concerned, there appear to be few new requirements, or at least few concrete requirements were generated, because Add. 3 is explicitly a $2 \mathrm{D}$ metafile. The model for STEP, computer graphics standards, and metafiles that seemed to get the greatest consensus involves a 3D file. PHIGS+ is seen as serving presentation and graphical requirements of STEP, and it was felt that a PHIGS+ workstation state capture file (as opposed to purely graphical capture) was the sort of metafile support needed. Meeting the metafile requirements of STEP would be accomplished through Addendum 2, and would involve some modification of the scope of Add.2.

The group discussed resources. It is agreed that there are not sufficient resources to process two projects (Add.2 and Add.3). The current WG3 Metafile RG members tend to be more interested in the 2D work (Add.3) than 3D work. This was not an official position, but an informal survey. It is clear that both projects cannot be progressed satisfactorily if more people are not available. A solution could involve collaborative work with WG2 for 3D metafiles.

It was generally agreed by the group that Addendum 3 should not be progressed as an addendum, at least not beyond the earliest stages. Rather, it and Add.1 should be folded into the CGM standard to produce a complete new document. This issue will be addressed by the WG3 Metafile RG when it commences work on the project.

There was time to work on the Add.3 baseline document during the meeting. The group divided into technical subgroups examining particular topics. As a result the baseline document was advanced sufficiently that the group feels it is suitable for the status of Working Draft. It was sent to the SC24 Secretariat for immediate circulation, with the hope of getting early comments that could be processed at Brazil.

\section{CURRENT STATUS \& PENDING MATTERS}

The NWI and Requirements Document are before JTC1 for a 3-month ballot. It is hoped that this will close before Brazil. Unfortunately some long delays have been reported in getting previous items through $\mathrm{JTCl}$, and this may adversely affect the Add. 3 schedule. 
The WG3 Metafile Rapporteur Group will have to ascertain the level of resource committment for Add.3, presumably at the Brazil meeting.

The NWI and Requirements Document commit Add. 3 to a certain level of coordination with the output of the Text Study Group and the Product Data Geometry Study Group. The final reports of these two grouns will have to be examined to see if any further specific requirements for Add.3 are implied.

Before the Working Draft stage is complete, the Metarile RG must finalize the agreed set of functions for Add.3 - this should be done at Brazil.

A Working Draft is currently being circulated for NWI comment. Early comment is hoped for by Brazil, so that work may take place at Brazil.

\section{COMMENTS ON THE STUDY PERIOD}

Herein are personal comments and observations of the rapporteur, both on the new procedures and on this Study Period.

The procedures outlined in N171 are excellent in principle. Some improvement was absolutely needed over the processes by which CGM itself was standardized, and by which Add.1 and Add.2 were undertaken. In the case of CGM perhaps 1-2 years of delay was incurred by fundamental disagreements over what was being standardized. In the case of Add.2, the scope and purpose have shifted several times, the effort is has not been sufficiently driven by agreed requirements, and in consequence little progress has been made. In the case of Add.1, these problems were potentially present again, but fortuitously there was reasonable consensus among those working on the project and good progress was made.

The result of the procedures is that 11 voting nations have endorsed the scope and purpose, and the high level functional definition of Addendum 3, and no voting nations are opposed. The hidden disagreements that have interferred with previous metarile work should not be a problem for Addendum 3.

There have been problems with the process however. The main problem is that it has taxed participants' resources too heavily. There were too many meetings and too much requirement for liaison for the resources available.

As a secondary effect of the resource shortage, certain steps specified in N171 were missed or passed as formalities. No formal input ever came back to the Study Period from the Requirements RG or the Reference Model RG before the Requirements Document and NWI went out to SC24 for ballot. This was due in one case to a liaison that was missed for lack of time and in the other case to a meeting which had to be cancelled.

The content of the Add.3 project was made dependent upon the output of two technology study groups. All three groups started off with a high activity level. One of the technology groups resulted in specific items that could be adopted by the Add.3 NWI; the other did not generate such. In both cases, the work of Add.3 Study Period cannot be complete until the final reports of the two groups are available, at Brazil, 16 months after the process commenced. 
The Add.3 Study Period was somewhat fortunate in the timing of events. Once the initial meetings happened in January, the meetings of the Reference Model group, the Requirements group, WG1 plenary, and the SC24 AG happened very soon after. Other projects following the procedures of $\mathrm{N} 171$ could be less fortunate and could incur significant delay. If all of the dependencies, those required by $\mathrm{N} 171$ and those required by technical liaison, had been rigidly observed it is easy to imagine that the process could take significantly longer than this Study Period.

In the balance the process was beneficial. New standards should be driven by agreed requirements and should have consensus on scope before work begins. However given the current environment and pressure on resources the procedures need to be simplified and streamlined. 

Attachment 6 :

Addendum 3 working Draft 



\section{ANSI X3H3}

Information Processing Systems .-

Compurer Graphics -.

Metarile for the Storage and Transfer

of Picture Description Information

Past 1

Functional Specification

(Clause 3)

Addendun 3

Draft Document 2.0 

Page 6

Sub-clause 3: add or change the following enuries:

colour model: A specification of a 3D colour coordinate system and a 3D subspace in the coordinate system within which each displayable colour is represented by a point. Some colour models include a fourth, redundant dimension to allow the independent representation of black. For the purpose of ISO 8632 colour model refers to one of RGB, CIE L* $u^{*} v *$, or CYMK.

colour selection mode: Indicator as to whether colour selection is to be direct (by specifying a colour value) or indexed (by specifying an index into a table of colour values). See COLOUR VALUE.

colour representation method: Indicator as $w$ which of three colour models (RGB, CIE $L^{*} u^{*} v^{*}$, CYMK) or spot colour is being used to represent colour values. See COLOUR VALUE, SPOT COLOUR.

colour value: The character string (for spot colour) or values of the point components (for colour model) describing a colour.

RGB: An additive colour model, well matched to colour display monitors, whose values are derined by the nomalized weights of Red, Green, and Blue components.

CIE L"u*v*: A colour model derining an absolute colour space based on colour matching experiments whose components are $L *$ (Lighuness) and $u * v *$ (Chromaticness).

CYMK: A subractive colour model, common in the printing industr;, which has cyan, magenta, yellow, and black components.

Reference Colour Model: Basic colour model within CGM relative to which relationships to specifiable colour models (RGB, CYMIK, and CIE $\left.L^{*} u^{*} v^{*}\right)$ are calibrated. The refcrence colour model is defined by the CIE 1931 sundard colorimetric sy'stem (XYZ).

spot colour: An exactly defined colour with a registered name. 



\begin{abstract}
ANSI X3H3
Information Processing Systems .-

Computer Graphics -

Metarile for the Storage and Transfer

of Picture Description Information
\end{abstract}

Part 1

Functional Specification

(Clause 4)

Addendum 3

Draft Document 2.0 
Subclause 4.3: Add the following to the list of elements given in the first paragraph of this clause:

COLOUR REPRESENTATION METHOD

FONT DEFINITION

FONT ATIRIBUTES

CHARACTER KERNING MODE

CHARACIER KERNING TABLE

Page 10

Subclause 4.32.1: Add the following to the list of elements given in the second paragraph of this clause:

COLOUR REPRESENTATION METHOD

CONIC ARC

CONIC ARC TRANSFORMATION MATRIX

PARAMETRIC SPLINE CURVE

RATIONAL B-SPLINE CURVE

RATIONAL B-SPLINE CURVE CLOSED

Page 11

Subclause 4.3.2.2. Add the following to the list of elements given in the second paragraph of this clause:

COLOUR REPRESENTATION METHOD

FONT DEFINITION

FONT ATIRIBUTES

CHARACTER KERNING MODE

CHARACIER KERNING TABLE

Page 11

Add the following as subclause 4.3.4

\subsubsection{Font Elements}

The FONTMEIRIC DEFINITION element is provided 10 all permit exact typographic placement of the character glyphs specified within a text suring. Using FONTMETRIC DEFINITION, the initial character in a text string would be placed at the specified coordinates, and each subsequent character would be offset by the width and right side bearing of the previous character and by its own left side bearing. If character keming is also in effect. then the inter character space would also be adjusted by the specified kem value. The character height and offser from the baseline are used to determine interline string placement, ascent, and descent.

The FONT ATIRIBUTES element can be used $w$ select a best fit font if an exact match is not available on a specific device.

Page 12

Subclause 4.4.2, furst line: 
Change "direct (RGB) colour"

inw "direct colour"

Page 14

Subclause 4.4.6, second paragraph, first line:

Change "RGB"

into "a direct colour"

Page 14

Subclause 4.5.2: add the following to the end of the subclause

The IMAGE APERT.RE is not affected by the setung of the CLIP INDICATOR element. Aperture setuing for pel array elements is assumed 10 be always on:. The default IMAGE APERTURE is listed in clause 6.

Add the following as subclause 4.5.3:

\subsubsection{Aperture.}

Selection of the region of interest within a pel array, whether clipped by the CLIP RECTANGLE or not, is accomplished using the IMAGE APERTURE. Since the IMAGE APERTURE 'mode' is always assumed to be 'on', the display of all pel array elements is alway's considered to be controlled by an aperure setuing. The default image aperture is listed in Clause 6. The IMAGE APERTURE element thus affects all subsequent pel aray elements that follow in the metifile until the aperture is overridden by the appearance of the next DMAGE APERTURE element.

Page 15

Subclause 4.6: add the following to the list:

CONIC ARC

COMPRESSED PEI ARRAY

IILED PEI ARRAY

PEI ARRAY REFERENCE POINT

PARAMEIRIC SPLDNE CURVE

RATIONAL B-SPLDNE CURVE

RATIONAL B.SPLNE CURVE CLOSED

Page 15

Subclause 4.6: add the following to The line elements are:" list:

CONIC ARC

PARAMEIRIC SPLINE CURVE

RATIONAL B-SPLINE CURVE

Page 16 
Subclause 4.6: add the following to "The filled-area elements are:" list:

\section{RATIONAI B.SPLINE CURVE CLOSED}

Page 16

Subciause 4.6: change "The cell array element is:" to "The cell array elements are:" and add the following to the list:

\section{COMPRESSED PEI ARRAY}

TILED PEI ARRAY

PEI ARRAY REFERENCE POINT

Page 16

Subclause 4.6.1.1: change subclause to read the following:

4.6.].] Description. There are two general line elements - POLYLINE and DISJOINT POLYLINE - four line elements relating to circles, ellipses and conic ares, and two elements that relate to spline curves.

Page 16

Subclause 4.6.1.1: change the end of the subclause:

CONIC ARC

generates a parabolic, hyperbolic or elliptical arc; the parameterization of the arc(s) is described in 5.6.X.

XXX SPLDE CURVE generates a single spline curve; two separate parameterizations of the spline curve are possible; these are described in 5.6.X and 5.6. $\mathrm{X}+1$

Page 16

Subclause 4.6.1.3: change the last sentence of the subclause to read:

The ARC and SPLDE primixives...

Page 17

Subclause 4.6.4.1: change the second sentence of the subclause to read:

"In addition there are seven elements that..."

Page 18

Subclause 4.6.4.1: add the following to the end of the subclause:

RATIONAL B.SPLINE CURVE CLOSED

generates a closed B-spline curve the set of syles is the same as for POLYGON.

Page 18 
Subclause 4.6.4.3 2nd paragraph: change the senience to read:

The circular, elliptical and B-spline fill primitives..."

Page 18

Add the following as subclause 4.6.5.1:

4.65.1 Pel Array Elements

COMPRESSED PEI ARRAY

represents a rectangular binary image compressed according to the CCIII T4 or T6 facsimile recommendations: two parameterizations are possible, one corresponding to the normal facsimile-size image, and a tiled format for large images; the elements are described in 5.6.X and 5.6.X +1 .

TIED PEI ARRAY

represents a series of equally sized, conuguously positioned individual raster images, or "tiles". The furst tile is placed at the PEI ARRAY REFERENCE POINT, and then the tiles are placed in sequence in the direction of the pel path and line progression as shown in figure $X$. The tiles are numbered by the lile index contained in the pel array identifier parameter of each tile.

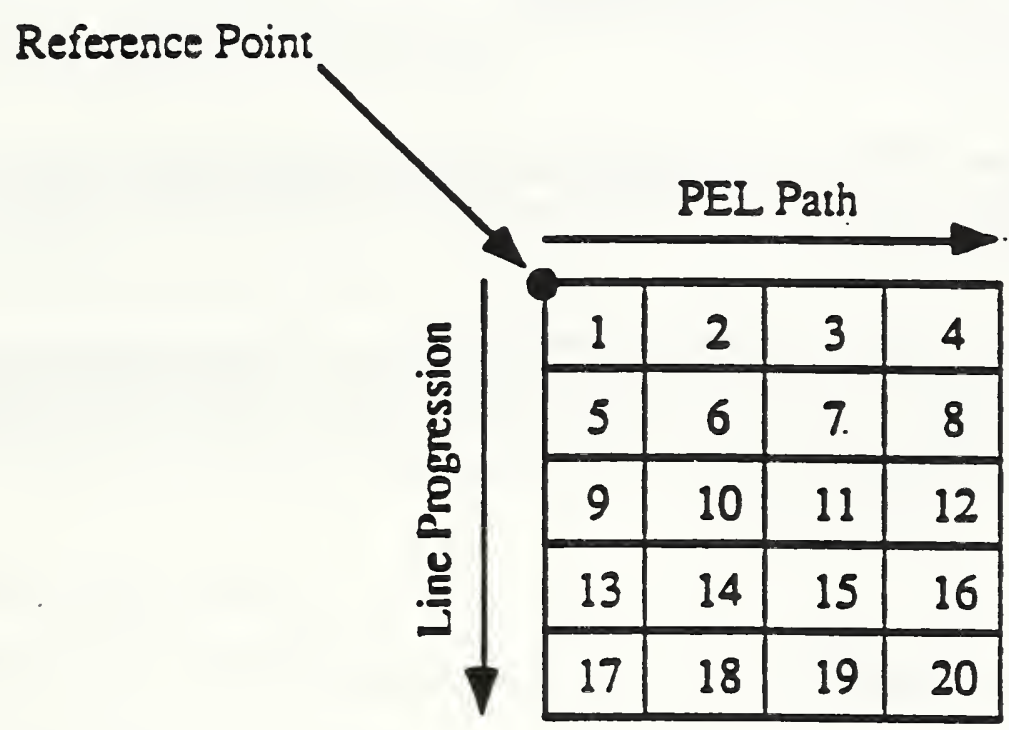

Figure $X$ Ordering and layout of tiles by index

4.65.1.1 Atribuses. The orientation and dimensions of the pel array elements is controlled by the PEL ARRAY ORIENTATION and PEI ARRAY DIMENSIONS elements. The PEI ARRAY ORIENTAIION element specifies the the direction of the layout of pels, i.e. the pel path, in 90 degree increments, relative $\omega$ the VDC axis. This element also specifies the direction of the layout of lines of pels, i.e. the line progression, in 180 degree increments relative to the pel path direccion. The pel path and line progression are constrained 10 be at right angles 10 one another.

4.65.12 Positioning. The position of a pel array element is defined by the PEL ARRAY REFERENCE PODT element. The reference point element affects the position of all pel aray elements that follow it in the melarile, unil it is overridden by a subsequent PEL ARRAY REFERENCE POINT EIEMENT. The 
visual effect on whatever might already be positioned at or near a given reference point by the overlay of a pel array element is implementation-dependent.

4.65.1.3 Tiling. The tiling mechanism specified is based on the Tiled Raster Interchange Format work that has been developed relative to MIL-STD-1840 and ISO 8613 Par 7. The TILING MODE control element, when "on", indicates th:", all subsequent COMPRESSED PEI ARRAY elements are th be considered individual tiles within the tiled image until the a subsequent TILING MODE element sets the mode 20 "off". If the number of "tiles" defined while tiling mode is "on" is less than the number indicated by the TILED PEI ARRAY element, then the missing tiles are treated as encoded as "null background". The tiling offset parameter defines the position of the actual pel array within tile space, relative to the PEL ARRAY REFERENCE POINT. All tiles cover a portion of the pel array, the portions of the tile space outside of the tile array are artifacts of tiling and contain no information.

Page 19

Subclause 4.6.7: add the following after the subclause:

\subsubsection{Conic Arc Element.}

A Conic Are is a bounded connected portion of a parent conic curve which consists of more than one point. The parent are is either an ellipse, a parabola, or a hypcrbola.

4.6.8.d Parameterization. A conic are is defined by the end points and the six parameters. The conic arc itself is defined by the six parameters in the following equation:

$$
A\left(X^{2}\right)+B X Y+C\left(Y^{2}\right)+D X+E Y+F=0
$$

This parameterization assumes that VDC space is 4 quadrant cantesian coordinant space. The CONIC ARC IRANSFORMATION MATRIX element is then used to properly position the are in the quadrant of VDC space derined by the VDC EXIENT element.

4.6.8.2 Geometric Concepts. The conic are is derined by the star point, end point and the six parameters A.F. To detemine the form of the conic arc, the quantities Q1, Q2 and Q3 are defined as follows:

$$
\begin{array}{ll}
\mathrm{Q} 1=\text { deteminant of } & \begin{array}{l}
|\mathrm{A} B / 2 \mathrm{D} / 2| \\
\mid \mathrm{B} / 2 \mathrm{C} \\
|\mathrm{D} / 2 \mathrm{E} / 2 \mathrm{~F}|
\end{array} \mid \\
\mathrm{Q} 2=\text { deteminant of } & \begin{array}{l}
|\mathrm{A} B / 2| \\
|\mathrm{B} / 2 \mathrm{C}|
\end{array} \\
\mathrm{Q} 3=\mathrm{A}+\mathrm{C} &
\end{array}
$$$$
\text { If } Q>0 \text { and }(Q 1 * Q 3)<0 \text {, then the are is elliptical; }
$$$$
\text { if } \mathrm{Q} 2<0 \text { and } \mathrm{Q} 100 \text {, then the are is hyperbolic: }
$$$$
\text { if } \mathrm{Q} 2=0 \text { and } \mathrm{Q} 100 \text {, then the are is parabolic. }
$$

In the case where the conic arc is elliptical, to distinguish the arc in question from its compliment, the direction of the are with respect to VDC space must be from start point to end point in a counterclockwise direction.

In the case where the conic arc is parabolic or hyperbolic, the parameterization derines a unique portion of the parabola or a unique porion of a branch of the hyperbola, thus the direction is irrelevant.

\subsubsection{Spline Curve Elements}


The elements described in this section were derived from IGES V3.0, and are specialized for the case of two dimensions.

4.6.9.1 Parametric Spline Curve. The parametric spline curve is a sequence of parametric polynomial segments. The definition of his class of curves is gencralized to allow for the representatioi of many different parameric spline curves using only one element. The following curve types have been assigned:
1: linear
2: quadracic
3: cubic
4: Wilson-Fowler
S: modified Wilson-Fowler
6: B spline

4.6.9.1.1 Perameterization. The degree of convinuity parameter indicates the smoothness, or continuity of the curve with respect $w$ arc length. The curve can either be continuous at all break points, continuous and have slope continuity at all break points, or be continuous and have both slope and curvature continuity at all break points.

The number of segments parameter is the number of polynomial segments to be used to define the curve. Each $X, Y$ polynomial segment is evaluated using the eight polynomial coefficients associated with that segment (AX,BX,CX,DX,AY,BY,CY,DY). Each segment is delimited by its respective breakpoint.

4.6.9.1.2 Geometric Concepts. The following cubic polynomial equations will seturn the coordinates of the points of the $i$-th segment of the curve. Note that the coefficients $D$, or $C$ and $D$ will be zero if the polynomials are of degrees 2 or 1, respecuvely:

$$
\begin{aligned}
& X(u)=A X(i)+B X(i)(s)+C X(i)\left(s^{2}\right)+D X(i)\left(s^{3}\right) \\
& Y(u)=A Y(i)+B Y(i)(s)+C Y(i)\left(s^{2}\right)+D Y(i)\left(s^{3}\right) \\
& \text { where } T(j) \Leftrightarrow u<=T(i+1), j=1, \ldots N \text { and } s=u-T(i) \text {. }
\end{aligned}
$$

The teminate point and derivatives are derived without computing the polynomials by evaluating the Nith polynomials and derivatives at $\mathrm{u}=\mathrm{T}(\mathrm{N}+1)$. These data, divided by the appropriate factorial (i.e. the second derivative divided by $2 !$, the third by $3 !$ ), are used as the $N+1$ or terminate point values.

\subsubsection{Rarional B.Spline Curve.}

4.69 2.1 Parameterization. The Ravional B.Spline curve is parameterized where:

$$
\begin{aligned}
& \text { sar_param < } 1<\text { end_param, } \\
& T(0)<=\text { sar_paran < end_param }<=T(N)
\end{aligned}
$$

Thus for any parameter value $t$ between $T(0)$ and $T(K+1)$, the sum of the basis functions satisfies the following idencity:

$$
b O(t)+b l(t)+\ldots+b K(t)=1 \text {. }
$$

If all of the weights in the weight list are not equal, then the equation type is ratinnal. Otherwise, if all of the weights are equal, then all of the weights cancel, the denominators sum 10 one and the equation type is polynomial.

4.6.92.2 Geometric Concefts. The parametric equation governing the definition of the rational B-spline curve is shown in the following expression: 


$\frac{W(0) * P(0) * b O(t)+W(1) * P(1) * b l(t)+\ldots+W(K) * P(K) * b K(t)}{W(0) * b O(t)+W(1) * b l(t)+\ldots+W(K) * b K(t)}$

where $W(i)$ are the weights, $P(i)$ are the control points and $b i(t)$ are the basis functions. The basis functions are all non-neg?ive piecewise polynomials determined by the degree and the knist sequence. The knot sequence is a non-decreasing lis of real numbers $T(-M)$.... $T(0)$,...T(K+1). Each basis function is suppored for the knot sequence interval $[T(i-M), T(i+1)]$. where $M$ is the degree of the basis function. Between any two adjacent bnot values, the corresponding basis function can be expressed as a single polynomial. The basis functions are derined as follows:

Let $N(t \operatorname{ti}-m, \ldots, \ldots i+1)$ denote the B-Spline basis function of degree $m$ supporzed on the interval [u-m,titl]. The functions of degree $d$ are defined with respect to those of $d-1$, as in the following:

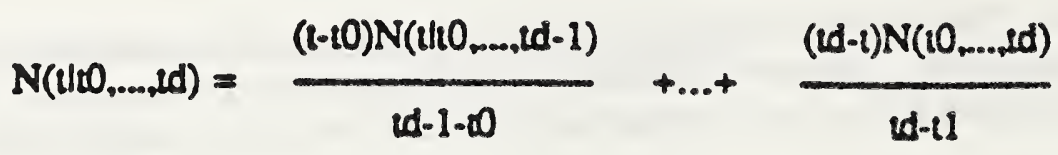

Since the denominators will be zero $(0)$ in some cases, the convention $0 / 0=0$ is adopted for this definition.

\section{X.X Compound Line}

The compound line elements consist of the two elements, BEGIN COMPOUND LINE and END COMPOUND LINE. These elements permit the definition of a line that consists of a number of distinct elements, such as straight lines and arcs, which is treated as if it were a single line element. Thus, for example, line style would apply without change or interruption past a straight line segment onto a following are segment. Likewise, the ends of the various component elements of the compound line are not treared as line end caps but rather as line joints.

\section{X.X Compound Text Path}

The compound rext path elements consist of the two elements, BEGIN COMPOUND TEXT PATH and END COMPOUND TEXT PATH. It is functionally identical to Compound Line, except that it is used as a base line for lexi placemen, rather than drawn by an interpreier.

The Compound Text Path permits arbitrary, complex placement of text. Each font symbol is placed with its reference point and alignment according to a tangent to the Compound Text Path. This implicit tangent is the logical base line for each character cell. If a symbol's reference point aligns with the junction of tw'o line elements of the Compound Texi Path, the logical base line is the line perpendicular to the perpendicular bisector of the langents of both elements, passing through the reference point. Positioning of subsequent symbols is based upon the distance between symbols assuming a straight base line, but wrapped long the generalized curve of the Compound Text Path. If there is more text than path, the path for the excess cexs is the straight line described by the tangent to the last element of the Compound Text Path.

\section{X.X Picture Composition}

The picture composition elements consist of BEGIN CLIP REGION, END CLIP REGION, BEGIN SHIEID REGION, END SHIEID REGION, CLIP INDICATOR, and SHIEID INDICATOR.

The concepts of clip and shield regions are complementary. The clipping process discards everything that is visually outside the clip region whereas the shielding process discards everything that is inside the shield region. Whether elipping and shielding are in effect is determined by the respective seutings of the CLIP INDICATOR and SHIEID INDICATOR (each is either ON or OFF). 
Due to being able to define what amounts 10 closed figures for these regions, the clip region and shield regiens can each provide a clipping and a shielding capability at the same time. For example, a "polygon with a hole" has an ourer boundary and an inner boundary. The "Illled area" of such a clipping region would be preserved with the area outside the "filled area" (including the contents of the hole") being removed from the picare. The shielding region has a complementary interpretation: the "Iilled area" itself is removed from the picatre.

Note that the shielding effect of a clip region "hole" is independent of the SHIEID INDICATOR and likewise, the elipping effect of a shield region "hole" is independent of the CLIP INDICATOR.

Page 37

Subclause 4.7.7: replace the current text body with the following:

The CGM must be able to represent colours in a manner suitable to a broad spectrum of graphical devices and systems. Towards this goal, the CGM uses three colour models (RGB, CIE L" $u^{*} v^{*}$. CYMK) and spot colour. The Metafile Deseripror element COLOUR REPRESENTATION METHOD, allows metarile generators to specify which ane is being employed.

The RGB additive colour model uses a 3-tuple of values providing the normalized weight of the red $(R)$, green (G), and blue (B) components of the desired colour. This model is used in colour monitors, film writers, and input seanners.

The CIE L "u* uniform colour model uses a 3-uple of values providing the nomalized luminance $(L *)$, red-green chromaticness ( $\left.u^{*}\right)$, and blue-yellow chromaticness $\left(v^{*}\right)$ components of the desired colour. The advantages of the model over the others are (1) it separates chromaticness from luminance (allowing for easy monochrome display), (2) it is a uniform space for small colour differences (the Euclidean distance between two points in this model is more or less proporional to their perceived difference), and (3) it is an absolute colour model based colour matching experiments (thus being device independent and not requiring colous correction).

The CYMK subtractive colour model uses a 4-uple of values providing the normalized weights of the cyan $(C)$, yellow $(Y)$, magenta $(M)$; and black $(K)$ components of the desired colour. This model is used by printers and Graphics Ars drum seanners. In theonj, cyan, magenta, and yellow are meant 10 correspond to the red, green, and blue of the RGB model. In practice, actual inks used for colous printing only approximate this criterion. Black ink is added 20 atuin a greater dynamic range than is possible with three colours alone. In paricular, it allows the attainment of a much richer black than would be possible using only the furst three inks.

Spot colour uses a character string representing a registered or private colour name (similar in format to named fonts). Use of the former is recommended for mesafile transporability, because registration ensures unique naming of colours. Spot colours are registered in the ISO International Register of Graphical Items, which is maineained by the Registration Authority. When a spot colour has been approved by the ISO Worbing Group on Computer Graphics, the colour name will be assigned by the Registration Authority. Each registered colour will be specisied in lerms of its CIE L "u"v" coordinates.

The CGM provides two mechanisms for colour selection: 'direet' and 'indexed'. In 'direct' colour selection, the colour is specified by providing values for its normalized components (colour model) or by providing the character suring defining its name (spor colour). (The term 'direct colour value' will refer to any direet colour specifier, and the term 'direct colour model value' will refer only $w$ a direct colour specifier of a colour model (as opposed io spot colour)). In 'indexed' colour selection, the colour is specified by an index into a uble of direa colour values. Selection of one of these mechanisms may be done by an element in each Picure Descriplor.

For 'indexed' colour selection, the COLOUR TABLE attribute element is provided for changing the contents of the colour table. This element may appear throughout the picure body. However, the effect of changes in the colour vable on any existing graphical primitive elements that use the affected indices is not addressed in this Standard. 
Direct colour model values are either a 3-tuple or 4-tuple of values providing the normalized weight of the desired colour components. In the abstract, each component is nt zhalized to the continuous range of real numbers $[0,1]$. In a metarile, direct colour model value components are integers, and the Metarile Descriptor element, COLOUR VALUE EXTENT, allows metafile generators to specify the minimum and maximum metarile direct colour model values for normalization.

To address the problem of colour matching physical devices, the CGM provides a mechanism for colour calibration. The device space for a paricular inpuvoutput device consists of the quantities that device uses for the measurement or the rendition of colour. Typical examples of devices and their associated device spaces are:

- colour monitors - this space is the intensities of the Red, Green, and Blue phosphors;

- colour film recorders - this space is the Red, Green, and Blue command values used for exposing the film:

- colour input seanners - this space is the values measured through the Red, Green, and Blue filters of the seanner,

- ink jet writers and thermal printers - this space is the ink values Cyan, Magenta, Yellow, and Black deposited onio paper.

- colour printing press - this space is the ink values which appcar on separation films, which are later to be rendered on presses or proofing systems.

For all the kinds of devices listed above, it is imporant to appreciate that each of them has a well-derined meaning only in terms of the paricular device with which it is used, but only an approximate meaning in terms of appearance. For example, if two pictures specified in tems of RGB are displayed on different monitors, the colours of the resulting images will not look identical. This is because no two monitor/display processor combinations are identical. If the two display systems of the same space are supplied by the same vendor, the images can be close enough for all but the most exacting applications: nonetheless, the difference does exist and must be taken into account. Similar considerations hold for the input seanner and ink spaces, with the extra problem that the viewing environment also affects the appearance.

Calibration is achieved by the imaging system converting a colour in the specified colour model to a colour in the reference colour model, and then convering to from the reference model to the device space for its inaging device. This applies for both input and output devices. Although this is conceptually two operations, it can be implemented as one transformation. These transformations require calibration data. The CGM does not allow for the interchange of calibration data. Instead, default values have been chosen to represent recognized standards for devices which use these modcls. 


\begin{abstract}
ANSI X3H3
Information Processing Systems -o

Computer Graphics -

Metafile for the Storage and Transier of Picture Description Information
\end{abstract}

Par 1

Funcuional Specißjcation

(Clause 5)

Addendum 3

Draft Document 1.3 

Page 41

Sub-clause 5.1: Table of data type abbreviacions:

Replace

CD Colour Direct Three-upple of non-negative real values for red, green, blue colour intensities.

with

MD Model Direct Three-tuple or four-tuple of non-negative real values for colour definition within one of the three suppored colour models.

CD Colour Direct String or Model Direct (as determined by COLOUR REPRESENTATION MEIHOD).

Pages $47-48$

Sub-clause 5.3.7: Replace the description as follows:

The precision for operands of data type model direct (MD) is specified for subsequent data of type MD. The precision is defined as the field width measured in units applicable to the specific encoding. Although the form of the parameter is encoding dependent, the parameter is a single specification that applies to each or all of the three or four components of paraneters of type CM. The precisions of the individual components are not independendy and differenty specifiable by this element.

Pages $48-49$

Sub-clause 5.3.10: Replace the description as follows:

The parameters represent an extent which bounds the direct colour model values that will be encountered in the metarile. It need not represent the exact extent of colour model values contained in the metafile.

Page 54

Subclause 5.3: Add the following Metarile Descriptor Elements:

\subsection{COLOUR REPRESENTATION METHOD}

\section{Parameters:}

colour representation method (one of: RGB, CIE L*u*v*,CYMK, spot colour) (E)

Description:

Four methods of colour representation are supponed: by one of three colour models (RGB, CIE L"u*v*. CYMCK) or by spot colour (names).

Only one colour representation method may be used within a metafile. The method may be defaulted or explicilly set with the COLOUR REPRESENTATION METHOD element. All oceurrences of colour-seuing elements (AUXILIARY COLOUR, LNVE COLOUR, MARKER COLOUR, FII COLOUR, EDGE COLOUR, TEXT COLOUR) as well as the colour lists of CELI ARRAY and PATIERN TABLE shall be in the current method. If used, COLOUR REPRESENTATION MEIHOD shall be in the Metarile Descriptor, after BEGIN METAFILE and before BEGIN MEIAFI E BODY. 


\section{References:}

4.7.7

\subsection{FONT ATTRIBUTES}

\section{Parameters:}

font number (IX)

number of attribute pairs (I)

List of: pairs of

Aluribute type, Atribute value (S)

where atribute values must be on of:

typeface name (S)

family name (S)

typeface general class (DX)

typeface sub-class (DX)

typeface specific group (DX)

posture (one of: postures defined by ISO/IECDIS 9541-5, 6.6)

weight (one of: weights derined by ISO/IECIDIS 9541-6.6.7)

proporionate width (one of: see ISO/IECIDIS $9541 \cdot 5.6 .8$ )

\section{Description:}

The order of the attribute values indicates the relative imporance of the attribute for font substitution. A missing value indicates no imporance to be placed on the value. The following auribute types have been assigned:

typeface name

family name

typerace general class

typerace sub-class

typeface speciric group

posture

weight

proporionace width

The fort atributes provide a more detailed description of the fonts defined in the FONT LIST element so as to enable rational font matching in the event of the inability $t 0$ exactly match a font from the font name specified in the FONT LIST element.

The font number will correspond to the font index defined in the FONT IST element.

The typeface name is the name of the font typeface. Note that a typeface name implies paricular values for the family name, weight posture, and proportionate width.

The typeface general class is the most general grouping of fonts with similar characteristics. Typeface sub-classes are groupings that identifies the less general characteristics and stars to eategorize typefaces inw similar designs. Typeface specific groups are typeface groupings with very distince and unique characteristics. Typefaces categorized to the typeface specific group level sar w show similar characteristics that makes them reasonably eligible $w$ be substituted for each other. The assigned fonis groups, and their atributes, are defined by the normative annex $A$ of ISO $9541 \cdot 5$.

The posture of a font may be one of the following: 
1: upright

2: obliquely slanted clockwise from the verical, with no other design adjustments

3: obliquely slanted counter-clockwise from the verical, with no other design adjusuments

4: inalic, slanted clockwise and the design adjusted for better appearance

The font weight is a measure of the boldness of the font. Assigned values are:

1: vitra light

2: extra light

3: light

4: semi light

5: medium

6: semi bold

7: bold

8: extra bold

9: uitra bold

The proportionate width is an indication of the ratio of character height 10 character width, and may be one of the following:

1: uitra condensed

2: extra condensed

3: condensed

4: semi condensed

5: medium

6: semi expanded

7: expanded

8: extra expanded

9: ultra expanded

In the preceding lish ultur condensed has the highest ratio of character height to character width, and ulua expanded has the lowest ratio of character height to character width.

\section{References:}

\subsection{FONTMETRIC DEFINITION}

\section{Parameters:}

font index $(D X)$

character index (C)

left bearing (VDC)

right bearing (VDC)

characer widh (VDC)

character height (VDC)

offset from baseline (VDC)

\section{Description:}

The fontmerric information for each character used in each font specified is defined by this element. If this element is used, then the fontmetric data for each character used in the metarile must be specified. Characters not used by the metanle may also be specified, but are not required.

\section{References:}




\subsection{CHARACTER KERNING MODE}

\section{Parameters:}

characles keming mode (one of: none, pair, sectored, track) (E)

Description:

Defines the kerning style, if any, for the metarile.

\section{References:}

\subsection{CHARACTER KERNING TABLE}

\section{Parameters:}

To be determined.

\section{Description:}

The data derined by this element will be dependant upon which, if any, kerning styles are supporred. In general, however, the information will be that which is required wo kem characters.

\section{References:}

\subsection{BEGIN GLYPH DEFINITION}

\section{Parameters:}

character index (IX)

\section{Description:}

BEGDN GLYPH DEFINITION delimits the beginning of a definition of a entity that will be treated as a single "compound primitive". The elemenis that make up the compound figure can be any combination of non-closed line elements such as POLYLINE. CIRCULAR ARC 3 POINT. CIRCULAR ARC CENTRE, EI IIPTICAL ARC, <new curve elemenLS, BEGN REQUIRED FEATURE, END REQUIRED FEATURE, and CLOSE FEATURE.

The IIgure defined by the component line primitives will form a character glyph, and will be assigned to the character code referenced by the value of the character index parameter.

The interior of the glyph (see 4.6.4.4) is filled according to the currens filled-area atrributes. The composite figure will be either a singie figure or a set of figures, which is filled according to the parity (odd ar even) algaritum described under the POL YGON element, with the exception that the transition from a vertex marked as a 'closure vertex' to the next point specified in the definition does nos constiwre a boundary to the fill algorithm. A vertex is marked as a 'closure veriex' by the use of the CLOSE CONTOUR element.

The individual figures of the set are nor filled individually. The figures in the set may be disjoint (as in the 'dot' in the letuer ' $i$ '), may create 'holes' (as in the interior of the leuter 'o'), or may overlap.

Refereaces: 


\subsection{END GLYPH DEFINITION}

Parameters:

None

Description:

END GLYPH DEFINITIOPN delimits the end of a compound outline definition of a character glyph.

\section{References:}

\subsection{BEGIN REQUIRED FEATURE}

\section{Parameters:}

Nose

\section{Description:}

BEGIN REQUIRED FEATURE delimits the beginning of a definition of a entity that will be treated as a single "compound primitive". The elements that make up the compound figure can be any combination of non-closed line elements such as POLYLINE, CIRCULAR ARC 3 POINT, CIRCULAR ARC CENTRE, EILIPTICAL ARC, CLOSE CONTOUR, and <new curve elements>. The figure may be a disjoint Iigure.

If used, BEGIN REQUIRED FEATURE shall occur within the scope of a BEGIN GLYPH DEFINITION block. The resulting composise figure will be a specific feature of the character glyph being derined which is required for proper sendering of the glyph (such as a serif).

References:

\subsection{END REQUIRED FEATURE}

\section{Parameters:}

None

Description:

END REQURED FEATURE delimits the end of a compound outline definition of a specific feaure of a character glyph required for proper rendering of the glyph.

\section{References:}

\subsection{CLOSE CONTOUR}

Parameters:

None

Description:

CLOSE CONTOUR delimits the end of a compound oudine of a character glyph. The last point specified is marked as a 'closure verrex'.

References: 
Page 55

Sub-clause 5.42, Iirst paragraph of description:

Change "red, green, and blue" into "direct"

Page 57

Sub-clause 5.4.7, first line of second paragraph of description:

Change "RGB" inco "a direct colour value"

Page 60

Subchuse 5.5: Add the following Control Elements:

\subsection{BEGIN COMPOUND LINE}

Parameters:

None

Description:

BEGIN COMPOUND LINE delimits the beginning of a definition of a entity that will have consistent line attributes and will be treated as a single "compound primitive". The elements that make up the compound line can be any combination of non-closed line elements such as POLYLINE, CIRCULAR ARC 3 POINT, CIRCULAR ARC CENTRE, ELLIPTICAL ARC, or Cnew curve elemeniss.

Refereaces:

\subsection{END COMPOUND LINE}

Parameters:

None

Description:

END COMPOUND LDE delimits the end of a compound line definition.

References:

\subsection{BEGIN TEXT PATH}

Parameters:

None

\section{Description:}

BEGN TEXT PATH delimits the beginning of a definition of a entity that will provide the path in which a text string will be drawn. The elements that make up the compound text path can be any combination of non-closed line elemenis such as POLYLINE. DISJODNT POLYLINE, 
CIRCUIAR ARC 3 POINT, CIRCUIAR ARC CENTRE, EILIPIICAL ARC, Or ONEW CUrVe elementss.

Once defined, the compound text path takes the place of the text path as defined by the TEXT PATH element and the CHARACTI $\mathrm{ORIENTATION} \mathrm{elements.} \mathrm{The} \mathrm{skew} \mathrm{of} \mathrm{the} \mathrm{characters} \mathrm{is}$ sill relative to that specified in the CHARACTER ORIENTATION element, but the placement of subsequent characters is along the compound text path instead of in a line along the character up vector or character base vector.

\section{References:}

\subsection{END TEXT PATH}

\section{Parameters:}

None

\section{Description:}

END TEXT PATH delimits the end of a compound lext path derinition.

\section{References:}

\subsection{BEGIN CLIP REGION}

\section{Parameters:}

None

\section{Description:}

BEGIN CLIP REGION delimits the beginning of a definition of a entity that will provide the clipping region. When CLIP INDICATOR is 'on' only the porions of graphics elements inside or on the boundary of the clipping region are draun. The elements that make up the clipping region can be any combination of closed or non-closed elements such as POLYLINE, DISIOINT POLYLINE, POLYGON, POLYGON SET, CIRCULAR ARC 3 POINT, CIRCULAR ARC 3 POINT CLOSE, CIRCULAR ARC CENTRE, CIRCULAR ARC CENTRE CLOSE, EIITPICAL ARC CLOSE, or <new curve elementss. The entity thus derined is essentially a closed figure whose boundary is used as the clipping boundary.

Once defined, the clipping region lakes the place of the clipping region defined in CLIP RECTANGLE.

\section{Refereaces:}

\subsection{END CLIP REGION}

\section{Parameters:}

None

Description:

END CLIP REGION delimits the end of a clipping region delinition.

\section{References:}




\subsection{BEGIN SHIELD REGION}

Parameters:

None

Description:

BEGIN SHIEID REGION delimits the beginning of a definition of a entity that will provide the shielding region. When SHIELD INDICATOR is 'on' only the porions of graphics elements outside of the shielding region are drawn. The elements that make up the shielding region can be any combination of closed or non-closed elements such as POLYLINE, DISJODNT POLYLINE, POLYGON, POLYGON SET, CIRCULAR ARC 3 POINT, CIRCULAR ARC 3 POINT CLOSE, CIRCULAR ARC CENIRE, CIRCULAR ARC CENTRE CLOSE, EILIPTICAL ARC CLOSE or snew curve elementss. The entily thus defined is essentially a closed figure whose boundary is used as the shielding boundary.

References:

\subsection{END SHIELD REGION}

Parameters:

None

Description:

END SHIEID REGION delimits the end of a shielding region definition.

References:

\subsection{SHIELDING INDICATOR}

Parameters:

shield indicator (one of: off, on) (E)

Description:

When SHIEID INDICATOR is 'off, shielding of graphical primitive elements is not required.

When SHIELD INDICATOR is 'on', only those portions of graphical primitive elements outside of the shielding region are drawn.

\section{References:}

\subsection{TILING MODE}

\section{Parameters:}

tiling roode (one of: off,on) (E)

Description:

When IILDNG MODE is "Ofr", subsequent COMPRESSED PEI ARRAY elements are to be treared as independent images displayed per the corresponding PEI. ARRAY ORIENTATION and PEI ARRAY DIMENSIONS atuibute elements, and positioned in VDC space via the PEI ARRAY REFERENCE POINT elemen. 
When the IIIING MODE is "on", each subsequent COMPRESSED PEI ARRAY element is treated as a single tile in a tiled image and indexed by the Pel Aray Identifies parameter. The PEL ARRAY ORIENTATION and PEI ARRAY DIMENSIONS elements describe the sizing and orientation of the entire tiled image. The dimensional information for the tiles in the image is contained in the IIIED PEL ARRAY element

\section{References:}

Page 68

Sub-clause 5.6.9: Add the following at the end of the third paragraph of the description:

Note that COLOUR PRECISION only applies to direct colour model values.

Page 77

Subclause 5.6: Add the following Graphical Primitive Elements:

\subsection{CONIC ARC}

Parameters:

$\operatorname{sar}$ point (P)

and point (p)

A.B,C.D.E,F (6R)

\section{Description:}

A conic are is drawn which is defined as follow's:

A conic are is defined by the end points and the six parameters. The conic are itself is defined by the six parameters in the following equation:

$A\left(X^{2}\right)+B X v Y v+C\left(Y^{2}\right)+D X v+E Y v+F=0$

where $(X v, Y v)$ are the horizontal and verical axes, respectively, of VDC space. In order for the conic arc to be processed correcly by the receiving system given the above representation, the conic arc entity must be positioned such that each of its axes is parallel to either the $X v$ axis or $Y v$ axis. The are is then positioned correetly in VDC space by using the value of the CONIC ARC TRANSFORMATION MATRIX elemenL

To determine the form of the conic are, the quanticies Q1, Q2 and Q3 are derined as follows:

\begin{tabular}{|c|c|}
\hline Ql = determinant of & 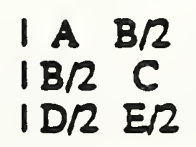 \\
\hline Q2 = determinant of & $\left|\begin{array}{cc|}A & \mathrm{~B} / 2 \\
\mid \mathrm{B} / 2 & \mathrm{C}\end{array}\right|$ \\
\hline
\end{tabular}

If $Q 2>0$ and $\left(Q 1^{\circ} \mathrm{Q} 3\right)<0$, then the are is an ellipse.

If $Q 2<0$ and $Q 100$, then the are is a hyperbola.

If $\mathrm{Q} 2=0$ and $\mathrm{Q} 1<>0$, then the are is a parabola. 
In the case where the conic arc is elliptical, 10 distinguish the are in question from its complement, the direction of the arc with respect 10 the definition space must be from start point 10 end point in a counterclockwise direction.

In the case where the conic are is parabolic or hyperbolic, the parameterization defines a unique porion of the parabola or a unique portion of a branch of the hyperb '3, thus the direction is irrelevant

The direction of the conic arc with respect to VDC space is determined by the original direction of the arc in definition space, in conjunction with the action of the CONIC ARC TRANSFORMATION MATRDX elemenL

\section{References:}

4.

\subsection{CONIC ARC TRANSFORMATION MATRIX}

\section{Parameters:}

matrix elements

if the VDC type is 'integer',

$R 11, R 12, R 13 R 21, R 22, R 23$

if the VDC type is 'real', $R 11, R 12, R 13, R 21, R 22, R 23$ (6R)

\section{Description:}

This element is intended to work in conjunction with the CONIC ARC element to transform the conic arc $w$ is final position and orientation in VDC space. The Transformation Matrix entity transforms the defining point coordinates by means of a matrix multiplication. This transformation is achieved by applying the matrix multiplication to the coordinates of the conic are.

The notation for this transformation is a: follows:

$$
\begin{array}{ll}
\mid R 11 R 12 R 131 & \mid \text { Xin | = | Xout | } \\
\mid \text { R21 R22R231 } & \text { Yin | | Yout | } \\
\mid \text { | } 11 &
\end{array}
$$

where $\mid R i j l$ is the transformation matrix, (Xin,Yin) is the coordinate to be transformed, and (Xout.Yout) is the coordinate resulting from the transformation. Both the input and output coordinare sysrems are assumed to be orhogonal, carresian and right-handed.

\section{References:}

4.

\subsection{PARAMETRIC SPLINE CURVE}

\section{Parameters:}

curve_type (DX)

H-degree of continuity (I)

N-number of segments (I)

T-break point list for polynomial $((\mathrm{N}+1) \mathrm{R})$

$X$ coordinate polynomial list (N sets of four) 
$A X, B X, C X, D X\left(\left(N^{*} 4\right) R\right)$

Y coordinate polynomial list (N sets of four)

AY,BY,CY,DY $\left(\left(N^{*} 4\right) R\right)$

\section{Description:}

The parameric curve to be drawn is defined as follows:

The parametric spline curve is a sequence of parametric polynomial segments. The parameterization shown above is generalized to allow for the representation of many different parametric spline curves using this one element. The eurve_type parameter indicates the type of paramerric curve as it was represented in the sending system before being converted to this generic form. The following curve types have been assigned:

1: linear

2: quadratic

3: cubic

4: Wilson-Fowler

5: modified Wilson-Fow'les

6: B.Spline

Values above 6 are reserved for regisuation and future standardization, and negative values are available for implementation-dependent use.

The degree of continuity parameter, $H$, indicates the smoothness, or continuity of the curve with respect to arc length. If $H=0$, the curve is continuous at all break points. If $H=1$, the curve is continuous and has slope continuity at all break points. If $H=2$, the curve is continuous and has both slope and curvature coninuity at all break points.

The number of segments parameicr, $N$, is the number of polynomial segments to be used to derine the curve. Each segment is derined by a cubic polynomial in $X$ and $Y$ that is evaluated using the eight polynomial coefficients associated with that segment; Ax, Bx, Cx,Dx, Ay, By, Cy,Dy. Segment $i$ is delimited by its knots, $T(i)$ and $T(i+1)$.

The paramersic spline curve is displayed with the current line attributes.

\section{References:}

4.

\subsection{RATIONAL B.SPLINE CURVE}

\section{Parameters:}

K-upper index of sum (I)

M-degree of the basis function (I)

equation_type flag (one of: rational, polynomial) (E)

T-knot sequence list $((\mathrm{K}+\mathrm{M}+1) R)$

W-weight list $((K+1) R)$

control point list $((K+1) P)$

san_parami,end_param (2R)

\section{Description:}

A two dimensional rational B-spline curve is drawn. The realization of this element is based upon the Rational B-Spline Curve encity of IGES V3.0. The specifications are identical except that the curve is constrained to two dimensions, i.e. the $Z$ Polynomial is assumed to be zero $(0)$. 
Valid values of the upper index and degree parameters are non-negative integers.

Valid values of the control points are such that no two adjacent points are coincident.

If all of the weights in the weight list $W$ are not equal, then the equation_type flag is set to Rational, otherwise the equation_type llag is set to Polyr amial.

\section{References:}

4.

\subsection{COMPRESSED PEL ARRAY}

\section{Parameters:}

PID-pel array. identifier (I)

Tencoding type (one of:T4,T6 LZW, Bitmap, null background, null foreground) (E)

P-pel path (one of:0,90,180,270) (E)

L-line progression (one of:90,270) (E)

S.pel spacing (VDC)

spacing racio $(R)$

N-number of pels per line (I)

NL-number of lines (I)

pel array (BS)

\section{Description:}

A compressed pel array image is derined as follows:

The pel array identifies, PID, is used as the tiling index when Tiling Mode is ON. When the Tiling Mode is OFF, the PID is used as an identifier to uniquely label the pel array for manipularion or access purposes.

The encoding type parameter, $T$, specifies the compression format used to encode the image. If $T$ is specified as "T4", the image is encoded according the one or two dimensional scheme defined in CCIIT Recommendation T.4 (Group 3 facsimile). If $\mathrm{T}$ is " T6", the inage is encoded according to the two dimensional scheme defined in CCITI Recommendation T.6 (Group 4 facsimile). I specisfed as "null background" or "null foreground" is used mainly when in viling mode io indicase that all pels in the tile are known to be background or foreground and the tile has no encoded contern. If either of these are specified, then the Pel Array itself will be a zero length or "null" binary string.

The pel path parameter, $P$, is the direction of progression of successive pels along a line relative to the VDC $X$ axis. This parametes, in conjunction with the pel spacing, $S$, and the number of pels per line, $N$, implicilly define the line position, length and granularity for each line in the decoded pel array. The spacing ratio is detuned as the ratio $M / S$, where $M$ is the distance in VDC units between two successive lines of pels, and $S$ is the Pel Spacing, which is the distance in VDC berween two adjacent pels in a line. Note that even if the VDC TYPE is Integer, the values of the Pel Spacing $S$ and the line spacing $M$ will be of type Real.

The line progression parameter, $L$, is the direction of progression of successive of pel lines and is expressed as a direction relative 10 P. $L_{0}$ in conjunction with the spacing ratio and the number of lines. NL. implicilly defines the size of the decoded image in the direction of $L$. The line spacing (LS) of the lines of pels ean be determined as follows:

\section{LS = spacing_ario * S}

The pel array itself is stored in either of the formars defined by $T$, encoded as a bit_stream. 
References:

4.

\subsection{TILED PEL ARRAY}

\section{Parameters:}

IID-riled pel aray ID (I)

ID-tiled pel array dimensions (2I)

NP-number of pels per tile line (I)

NL.number of lines per tile (I)

TO-iiling offset (2I)

\section{Description:}

A tiled pel array image is derined as follows:

The tiled pel array, IID, uniquely idencifies the riled image. Valid values of this ID are positive integers.

The riled pel array dimensions parameter, TD, consists of two positive integers corresponding to the number of tiles in the direction of the Pel Path and Line Progression parameters, respectively, of the PEI ARRAY ORIENTATION element. Multiplying the two TD integers together will give the total number of tiles contained in the tiled image.

The number of pels per tile line parameter, NP, specifies the tile dimension in units of Pel Spacing found in the PEI ARRAY DIMENSIONS element in the Pel Path direction of the PEL ARRAY ORIENTATION element, for each tile of the tiled image.

The number of lines per tile parameter, NL, specifies the tile dimension in units of line spaces, derived from the Spacing Ratio of the PEI ARRAY DIMENSIONS element, in the Line Progression direction of the PEI ARRAY ORIENTATION element, for each tile of the tiled image.

The tiling oftset parameter, TO. specifies the location of the pel array image within tiling space by defining the oftset of the first pel of the pel uray from the PEI ARRAY REFERENCE PODT. The offset is specified by two positive iniegers corresponding to the number of pel spaces in the Pel Path direction and line spaces in the Line Progression direction, respectively.

\section{References:}

\subsection{IMAGE APERTURE}

\section{Parameters:}

$X 1, Y 1, X 2, Y 2(41)$

\section{Description:}

The element derines the rectangular area of pels in the decoded pel aray that is to appear in the metafile picture. This element should immediately precede the Pel Array element 20 which it applies, as this element will affect all subsequent Pel Array elements unil another Image Aperture is explicilly defined.

The four iniegers form two coordinate pairs, $(X 1, Y 1)$ and $(X 2, Y 2)$ corresponding to the first and last pels to appear, respectively, where $X 1<=X 2$ and $Y 1<=Y 2$. For example, $(6,2)$ would specify the seventh pel in line 3 . given that $(0,0)$ specifies the first pel on the first line. 
These two comer pels, $(X 1, Y 1)$ and $(X 2, Y 2)$, derine the rectangular portion of interest of the Pcl Array image 10 be positioned in the mensile picture. The first pel delined by $(X 1, Y 1)$ above is to be positioned at the reference point of the Pel Array element, with the remainder of the image realized as per the Pel Spacing. Spacing Ratio, Pel Path, and Line Progression parameters of the Pel Array elemeni. Valid values of the IMAGE APERTURE parameters are non-negative integers.

\section{References:}

4.

\subsection{PEL ARRAY REFERENCE POINT}

\section{Parameters:}

reference_point $(P)$

\section{Description:}

The pel array reference point defines the position of the upper left-hand comer of the pel array element $w$ be displayed. If the pel path and line progression are thought of as vectors, the upper left-hand comer is derined as poins of origin for the two vectors.

\section{References:}

4.

\subsection{BOXED TEXT}

Parameters:

two points (2P)

Ilag (one of: not final, final) (E)

suring (S)

\section{Description:}

The two points specified represent diagonally opposite comers of a rectangle oriented parallel to the VDC axes. BOXED TEXT behaves as does TEXT, with the exception that the text is constrained to be within the reciangle delined by the two points.

The character codes specified in the string are interpreted to obtain the associnted symbols from the currently selected character sel. Characters are displayed on the view surface as specified by the texi auributes. Format effector control characters (such as CR, LF, BS, HT, VT, and FF) are permitred in a string but their interpreation is implementation dependent. Control characters used for character set invocation and designation (SI, SO, ESC, SS2, and SS3) are permitued according to the serring of CHLARACIER CODING ANNOUNCER.

The characters are dimensioned according to the height and width of the bounding box and are oriented according w CLARACTER ORIENTATION. The direction of character placement in the soing relative wo CHARACIER ORIENTATION is according wo TEXT PATH.

All tex in the string is displayed within the specified bounding box. If necessary, the values of the text amribures CHARACIER KEIGHT. TEXT PRECISION, and TEX. FONT INDEX which are nsed $w$ display the string are varied 20 force the text 20 completely fill the bounding box.

The llag parameter is used to permit changing the following text atuibutes and control elements within a soring which will be aligned as a single block: TEXT FONT DNDEX. TEXT PRECISION, CHARATER EXPANSION FACTOR, CHARACTER SPACING, TEXT 
If the flag is set 10 'not final', the character codes in the string parameter are accumulated, along with the current atribute seutings. In this case, only the atribute setting elements listed above are allowed between this element and the APPEND TEXT element. With the exception of the ESCAPE, no other metarile elements of any type are allowed. ESCAPE is permitted but has no sandardized effece

If the flag is set 10 'final', the string parameter constitutes the entire string to be displayed. It is this complete string 10 which the text bound box applies. The position of the suing is detemined by the bounding box. Text elements with a null string parameter are legal and may be followed by the allowed text awributes and APPEND TEXT as described above.

NOTE- TEXT PRECISION is included in the atributes which may be changed $t 0$ achieve the lext restriction because TEXT PRECISION controls the relationship between currently set vaiues of text atributes and the values actually used for display of a suring (the "realized" values). The realization of the text restriction required by the BOXED TEXT elements may mandate another mapping from requested to realize auribute values than would be allowable under the current TEXT PRCISION. Hence the requirements of the current TEXT PRECISION may have to be ignored 10 achieve the proper display of the BOXED TEXT elcment.

When the flag is 'not final' this element causes a state transition in the state diagram of figure 12 , in the PARTIAL TEXT sate.

\section{References:}

4.

\subsection{PATH TEXT}

\section{Parameters:}

Ilag (one of: not final, Iinai) (E)

sring (S)

\section{Description:}

The PATH TEXT element shall be immediately preceded by a compound line definition. The alignment point of the swing will be a point along the defined text path corresponding to the curreni TEXT ALIGNMENT value.

The character codes speciñed in the soring are interpreted to oblain the associated symbols from the ourently selected character set. Characters are displayed on the view surface as specified by the text arribules. Format effecror control characters (such as CR, LF, BS, HT, VT, and FF) are permilued in a string but their interpretation is implementation dependent. Control characters used for character ser invocation and designation (SI, SO, ESC, SS2, and SS3) are permilted according 'to the sering of CHLARACIER CODING ANNOUNCER.

The characters are dimensioned according to the CHARACTER HEIGHT and CHARACTER EXPANSION FACTOR and are oriented according 10 CHARACTER ORIENTATION. The direction of character placement in the suring relative to CHARACIER ORIENTATION is along the path defined within the scope of the preceding BEGDN TEXT PATH and END TEXT PATH elements. If the string length exceeds the length of the path, the characters of the string will continue $i$ be placed along the path defined by a vector whose tail is the last point of the path and whose direction is the direction of the path at the last point.

The flag parameter is used to permit changing the following text attributes and control elements within a string which will be aligned as a single block: TEXT FONT INDEX, TEXT 
PRECISION, CHARATER EXPANSION FACTOR, CHARACTER SPACING, TEXT COLOUR, CHARACTER HEIGHT, CHARACTER SET INDEX, ALTERNATE CHARACTER SET INDEX, TEXT BUNDLE INDEX, AUXILIARY COLOUR, and TRANSPARENCY.

If the Dag is set 10 'not final', the character codes in the string parameter are accumulated, along with the current atrribute settings. In this case, only the attribute seuting elements listed : 30 ve are allowed between this element and the APPEND TEXT element. With the exception of the ESCAPE, no other metafile elements of any type are allowed. ESCAPE is permitued but has no candardized effece.

If the Ilag is set 20 'Inal', the string parameter constitutes the entire string to be displayed. It is this complete string 10 which the text bound box applies. The position of the string is determined by the bounding box. Text elements with a null string parameter are legal and may be followed by the allowed text atributes and APPEND TEXT as described above.

NOTE. When the flag is 'not final' this element causes a state transition in the state diagram of Gigure 12, inw the PARTIAL TEXT state.

References:

4.

\section{S.6.X RESTRICTED PATH TEXT}

Parameters:

Ilag (one of: not final, fina) (E)

string (S)

Description:

The RESTRICTED PATH TEXT behaves as does PATH TEXT, with the exception that the texi is constrainct to be the same length as the path along which it is to be drawn.

The character codes specified in the string are interpreted to obtain the associated symbols from the currently selected character sel. Characters are displayed on the view surface as specified by the lexi atributes. Format effector control characters (such as CR, LF, BS, HT, VT, and FF) are permiued in a suring but their interpretation is implementation dependent Control characters used for characier set invocation and designation (SI, SO, ESC, SS2, and SS3) are permiuled according to the setting Of CHARACIER CODING ANNOUNCER.

The characters are dimensioned according to the CHARACIER HEIGHT and CHARACTER EXPANSION FACTOR and are oriented according to CHARACIER ORIENTATION. The direction of character placement in the string relative to CHARACTER ORIENTATION is along the path derined within the scope of the preceding BEGIN TEXT PATH and END TEXT PATH elements.

All text in the string is displayed along the specified COMPOSITE IEXT PATH. If necessary, and onily if necessary, the values of the lexi atuributes CHARACIER HEIGHT, CHARACIER EXPANSION FACTOR, CHARACTER SPACING, TEXT PRECISION, and TEXT FONT INDEX which are used 20 display the string are varied 10 force the lext 10 be the same length as the derining composite path. It is only the realized values of these auributes, used to display this singie string, which are varied. The method of varying the awributes is implementation dependent.

The flag parameter is used 20 permit changing the following text atuributes and control elements within a string which will be aligned as a single block: TEXI FONT DNDEX. TEXT PRECISION, CHARATER EXPANSION FACTOR, CHARACIER SPACING, TEXT COLOUR, CHARACIER HEIGHT, CHARACTER SET INDEX, ALTERNATE CHARACTER SET INDEX, TEXT BUNDLE INDEX, AUXRIARY COLOUR, and TRANSPARENCY. 
If the flag is set 20 'not final', the character codes in the string parameter are accumulated, along with the current atribute setuings. In this case, only the attribute setting elements listed above are allowed between this element and the APPEND TEXT element. With the exception of the ESCAPE, no other metarile elements of any type are allowed. ESCAPE is permitted but has no sandardized effect

If the flag is set 10 'final', the string parameter constitutes the entire string to be displayed. It is this complete string to which the text bound box applies. The position of the string is determined by the bounding box. Text elements with a null string parameter are legal and may be followed by the allowed iext amributes and APPEND TEXT as described above.

NOTE. TEXT PRECISION is included in the atributes which may be changed to achieve the text restriction because TEXT PRECISION controls the rehtionship between currently set values of text auributes and the values actually used for display of a suring (the "realized" values). The realization of the text restriction required by the RESTRICIED PATH TEXT elements may. mandate another mapping from requested to realize auribute values than would be allowable under the current IEXT PRCISION. Hence the requirements of the current TEXT PRECISION may have 10 be ignored to achieve the proper display of the RESTRICTED PATH TEXT element.

When the flag is 'not final' this element causes a state transition in the state diagram of figure 12 , into the PARTIAL TEXT staLe.

\section{References:}

4.

Page 95

Sub-clause 5.7.32: Add the following at the end of the third paragraph of the description:

Note that COLOUR PRECISION only applics to direet colour model values.

Page 97

Subclause 5.7: Add the following auribute elements:

\subsection{LINE TYPE DEFINITION}

\section{Parameters:}

linerype (DX)

dash unit selector (one of: VDC, mm, native device units, abstract) (E)

dash repeas length (R)

adaptive flag (one of: no, yes) (E)

list of dash elements (nl)

\section{Description:}

This element defines a linetype and associates it with an index for future reference. Parameter 'linetype' is the index of linetype being defined. The parameter 'list of dash elements' is the definition $t 0$ be associated with the index. The first element is a dash, second a space, etc. - the defined linetype is solid for 11 units, gap for 12 units, solid for 13 units, etc. N must be positive, and each dash element (I) non-negauve. $N=1$ means a solid line; $I=0$ interpreted as a dot.

The units of the 'dash repeat length' are specified by the 'dash unit selector' parameter. The value of 'abstract' indicates that the implementation may nomalize and map the sum of the dash pautem 
elements at its discretion. The 'dash repeat length' defines the length of one complete cycle of the dash pattern, measured in the units of 'dash unit selector'.

An "adaptive" linetype is one where every vertex falls on an inked portion of the line. This is accomplished in plotters by temporarily modifying the duty cycle for each line segment (ceiling funcion) such that there is always an integral number of repeats (and alı predefined linetypes have their gaps_array defined such that they begin and end with inked or "pen down" porcions).

\section{References:}

\subsection{HATCH STYLE DEFINITION}

\section{Parameters:}

hatch index (DX)

style indicaror (one of: parallel, crosshatch) (E)

hatch space units selector (one of: VDC, $\mathrm{mm}$, device units, abstract) (E)

angle (2R)

duty cycle length $(R)$

list of harch elements ( $\mathrm{nI}$ )

\section{Description:}

This element defines a hatch style and associntes it with an index for future reference.

The hatch index' parameter defines the index of hatch style being defined. The 'list of hatch elements' is an array that defines alternating line width and gap width - i.e., the width of a hatch line followed by the width of the space to the next hatch line. The center of the furst hatch line is matched up with PATIERN REFERENCE POINT, if implemented. 0 interpreted as thinnest line width available.

The hatch space units selector' specifies the units of 'duty cycle length'. It also controls the manner of uransformation of the hatching: If VDC, then the hatching transforms with segment transform and anisotropic transforms (as if hatching had done POLYLINES); otherwise, the batching is like "wallpaper" that shows through the polygon-shaped hole - everything is defined in device units and hatching performed in device space. The value of 'absuract' indicates that the implementation may normalize and map the sum of the list of hatch elements at its discretion. The 'duty cycle length' is measured perpendicular to the hatch line. The sum of hatch elements in the hauch element list is normalized to this distance before presentation of the hatch on the view surface.

The 'angle' parameter is measured in the units specilied by the 'hatch space units selector'. It consists of two components, $d x$ and dy, defining a vector.

\section{References:}

\subsection{LINE CAP}

\section{Parameters:}

line cap indicator (one of: buu, round, projecuing square) (E)

\section{Description:}

The line cap style is defined for subsequent line elcments. The line cap style determines the appearance of open endpoints (as opposed to interior verices) of line elements. The defined styles are 
buta cap:

round cap:

projecting square cap: the line is squared off at the endpoint, there is no projection beyond the endpoint.

a semicircular arc with diameter equal to the line width is drawn around the endpoint and filled in. The drawn line thus projects beyond the endpoine

the line is squared off at a distance equal to half the line width beyond the endpoint.

\section{References:}

\subsection{LINE JOIN}

\section{Parameters:}

line join indicator (one of: miter, round, bevel) (E)

\section{Description:}

The line join style is defined for subsequent line elements. The line join style defines the appearance of interior verices of polyline elements and of compound line elements. The derined styles are:

miter join:

the outer edges of the two adjoining line segments are extended unil they meet at a point

round join:

a circular are with diameter equal to the line width is drawn around the veriex between the adjoining segments and is filled in, producing a rounded comer.

bevel join:

the adjoining line segments are terminated with a butt cap, and the resuluing triangular notch is filled in.

\section{References:}

\subsection{EDGE CAP}

\section{Parameters:}

edge cap indicator (one of: buth, round, projecting square) (E)

\section{Description:}

The edge cap style is defined for subsequent edge elements. The edge cap style determines the spearance of open endpoints of filled area edges (such as may result from a mixture of visible and invisible edge segments). The defined styles are:

but cap:

round cap:

projecting square cap: the edge is squared off at the verex, there is no projection beyond the endpoint.

a semicircular are with diameter equal to the edge width is drawn around the endpoint and filled in. The drawn edge thus projects beyond the endpoint

the edge is squared off at a distance equal to half the edge width beyond the endpoint. 


\subsection{FONT SCORE TYPE}

\section{Parameters:}

seore type (one of: strikeout, underline, overscore, or none)

\section{Description:}

The score type is set the the value specified by the parameter.

The following seore type are assigned:
1: strikeout
2. underseore
3: overscare
4: none

The score type 'strikeout' is a line drawn through each character in the suring at the half verical alignnent position.

The score type 'underscore' is a line drawn below each character in the string at the 'bottom' verical aligmment posicion.

The score type 'overscore' is a line drawn above each character in the string at the 'top' verical alignment position.

The score type 'none' remove all scoring.

References: 


\begin{abstract}
ANSI X3H3
Information Processing Systems -

Computer Graphics -

Mecarile for the Storage and Transfer

of Picture Description Information
\end{abstract}

Par 1

Functional Specification

(Clause 6)

Addendum 3

Drafi Document 2.0

111 

Page 100

Clause 6: Add the following default specifications:

CONIC ARC IRANSFORMATION MATRIX:

identity marrix

PEI ARRAY CIIP RECTANGLE:

$(0,0)$ upper left, (N-1 L-1) lower right, where $N$ is the number of pels per line, and $L$ is the number of lines in the last

COMPRESSED PEI ARRAY elemenL.

PEI ARRAY REFERENCE POINT:

upper left-hand comer point of the default VDC extent

Clipping Region:

(BEGN/END CIP REGION)

VDC Extent

Shielding Region:

(BEGN/END SHIEID REGION)

VDC ExLen:

CLIP DNDICATOR:

On

SHIEID INDICATOR:

OfI

COLOLR : EPRESENTATION METHOD:

RGB 

Attachment 7:

Us Comments on Addendum 3 Working Draft 



\section{CGM Addendum 3 Working Draft}

\section{Introduction}

This document contains the US comments on the Working Draft of Addendum 3, document version dated 89/07/18. The commen ; are divided into three sections: the overall comments of the next section; a checklist comparing Add.3 Working Draft content to the NWI and requirements documents; and a section of detailed technical comments. In addition the US intends to bring contributions of revised text to the Olinda Metafile RG Meeting.

\section{Overall Comments}

\subsection{Schedule}

The schedule must be considered a requirement at least as strong as any of the other requirements. This may force compromises on technical content. In particular attempts to develop technical areas which will likely be lengthy, or will require liaison with other standards work which has a significantly longer timetable, should be postponed. The US believes that the work can and should be completed on the timetable projected in the NWI.

\subsection{Completeness of the draft}

The US believes that the current Working Draft is not sufficiently complete for DP registration. The functionality is incomplete with respect to that detailed in the NWI and Requirements documents, as pointed out below in "NWI/Requirements Checklist." The defaults are missing for many elements that need them. With the correction of these omissions, and the addition of the encodings, the Working Draft will be complete enough for DP registration and ballot.

\subsection{Compatibility with other SC24 Work}

As stated in the NWI the Addendum 3 work should coordinate with the SC24 work in APIs and Reference Models. However the Add.3 metafile has strong ties to common practice which is outside of the scope of SC24 standards as well - proprietary $\mathrm{CAD}$ databases, desktop publishing systems, page description languages, and graphic arts workstations to name a few. The US believes that there should not be arbitrary differences between this work and work on existing or new APIs. However the US defines the following priorities for possibly resolving conflicting requirements: schedule and identified functional requirements must have the highest priority.

\subsection{Document form}

Addendum 3 should ultimately be processed as a complete document, i.e., CGM plus Addendum 1 plus Addendum 3 should be merged into a single document for review and advancement. Because this is going to be fairly unwieldy, we think that this should be done at the stage that DP text is prepared and the DP ballot initiated. At the Working Draft stage it is easier to focus on functional completeness and correctness (and ignore document completeness and consistency) in the current 
format. If two DPs are anticipated, then the consolidation should happen at the second DP stage.

\subsection{Relationship to Addendum 1}

Addendium 3 should be a proper superset of Addendum 1. A legal Add.1 metafile should be a legal Add.3 metafile.

\subsection{Further Coordination with Improved Text Model Study Group}

The US recommends that any further work on Improved Text Model should take place in the Reference Models RG of WG1. In the meantime the work done so far, and useful extensions from current practice in the industry, should guide the improved text facilities of Add.3.

\subsection{Further Coordination with PDG Study Group}

No specific requirements for Add.3 have derived from the work of the PDG study group. The US believes that such requirements are most likely to be applicable to Addendum 2, the 3D metafile, when and if the scope of such is defined. If any specific requirements for $2 \mathrm{D}$ metafile support can be stated, then those should be observed in the work on Add.3. 


\section{NWI/Requirements Checklist}

The following list is extracted from the NWI and the requirements document for Addendum 3. Each item is examined and the state of the Working Draft is categorized for that item. All MISSING and INCOMPLETE conditions must be corrected before DP registration. The text and font facilities need significant work, and we have dealt with them in a separate section later in this document.

\subsection{Advanced 2D drawing capability}

a. curves

- Bezier curves: MISSING.

- Rational B-splines: OK.

- Parametric spline curves: OK.

- Conics, and conic arcs: OK.

b. additional line attributes

- line cap: OK.

- line join: OK.

- mitre limit control: OK.

c. composite line primitives: OIK.

d. user defined

- line types: OK.

- hatch styles: OK.

- marker types: MISSING.

e. arbitrary text path: OK.

f. filling mechanisms

- additional standardized hatch styles: MISSING.

- interpolated fill: MISSING.

- geometrically specified patterns: MISSING.

g. general transformations: $3 \times 3$ (and $2 \times 3$ ) transformation matrices to allow for affine and projective transformations: MISSING (except for conics).

\subsection{Improved text and font support}

Much work needẹ.

\subsection{Arbitrary boundaries for clipping and shielding}

OK.

\subsection{Additional color models beyond RGB}

a. CIE: OK (but check against latest draft of ISO 8613 Color Addendum).

b. CMYK: OK (but check against latest draft of ISO 8613 Color Addendum).

c. spot colour: OIK (but check against latest draft of ISO 8013 Color Addendum). 


\subsection{Additional image storage and transfer capabilities}

Coding/compression techniques: INCOMPLETE. (cleanup parameterizations, add local color precision, iff prove descriptions, address the issue of compression and color).

\subsection{Symbols}

a. external reference to libraries of named symbols: MISSING.

b. user-defined internal libraries: OK (adequately covered by Addendum 1). 


\section{Specific Technical Comments.}

\subsection{Bundles}

It is not described anywhere how the new attributes (line attributes, text/font attributes, etc) relate to the bundle concept of CGM and other SC24 standarrs. They should not be bundled. Predefined bundles are used for distinguishability where exact appearance does not count, and this is contrary to the whole reason for these "precise control" attributes. Settable bundles in static CGM only provide a macro mechanism, and the utility of such a mechanism does not justify the complexity of adding these to bundle definitions.

\subsection{Clipping to Arbitrary Boundaries}

The Add.3 requirements identify the need for clipping to arbitrary boundaries, and also the need for arbitrarily specified geometric fill (the latter has not been defined yet). The latter can provide the same capability as the former. Clause 4 should have text comparing and contrasting the features, and should furthermore address (and proscribe) possible recursive situations that could arise with the filling feature.

\subsection{Compound Clip Indicator}

There should be a COMPOUND CLIP INDICATOR which controls the clipping to arbitrary boundaries.

\subsection{Line Types and User-defined Line type}

a. Are the interior endpoints of a dashed line capped? Options are: yes, no, or selectable by a new option setting element.

b. The need for 'millimeters' and 'native device units' as modes for dash unit selector of user line type needs to be justified.

c. There should be a 'line width relative' mode for dash unit selector for line type definition.

d. There should be a LINE STYLE CONTINUA- TION function, which applies to both user defined and predefined dashed lines. The 'adaptive' parameter should be removed from user defined linetype. LINE STYLE CONTINUATION should minimally have the values "continue", "restart", or "adaptive continue". This parameter might be subject to registration of values as well, as some of the target application areas of Add. 3 have very specific requirements for how a dashed line is drawn with respect to dash lengths and inking of vertices.

e. There should be an INITIAL DASH OFFSET element, which specifies how far into the line pattern to start drawing a new polyline.

f. The text of User Linetype needs much clarification and improvement including definition of missing terms such as "N", "gap_array" and "duty cycle".

g. Which of the new Line Type functions affect edges. Is it intended to have equivalent functions for edges? 


\subsection{Shielding}

Multiple disjoint shield regions are needed. This could be achieved in a number of ways.

- The definition of a single region could allow for islands, muc' as POLYGON SET does for filled areas.

- or there could be multiple definitions of regions, each associated with an index and tied to its shield indicator by the index.

\subsection{Additional line caps}

A Line Cap style of 'triangular' is needed.

\subsection{Conics and conic ares}

a. Section 5.6.X states "the conic arc entity must be positioned such that each of its axes is parallel to either the $\mathrm{Xv}$ axis or $\mathrm{Yv}$ axis". Clause 4 does not have a similar restriction. If the latter is correct, is there need for the conic arc transformation matrix?

b. The assertion has been made, but not yet demonstrated, that the conics can all be represented by relatively simple splines. If this can be demonstrated then the conic arc entity should be removed in favor of a non-normative note describing how parabolas and hyperbolas can be realized with splines.

\subsection{General transformations}

The need for this was stated in the NWI and requirements. It is not addressed in the Working Draft, and it is not clear exactly what was intended by the requirements statement. This needs to be studied and clarified. In particular, do Segment and Copy transforms provide whatever capability is needed?

\subsection{Splines}

a. Unified Formulation. If formulations can be demonstrated which are consistent with the work of STEP, PHIGSt, and other related areas, and if these can be shown to easily subsume and support the formulations in the Working Draft, then these formulations should be used. Such claims have been made but not demonstrated.

b. Description. Tutorial information on the various splines and curves should be included in an annex.

c. The "curve type" and " $\mathrm{H}$ " parameters of the Parametric Spline curve are meaningless for the definition of the curve and should be removed.

d. The "equation type" flag should be removed - to get a polynomial one may put in an array of weights that sum to zero. A void array should be degenerate or illegal.

e. Closed curves. Clause 4 indicates a RATIONAL B-SPLINE CURVE CLOSED element, which is a Filled Area element. This is missing from Clause 5. 


\subsection{PEL ARRAY}

a. The compression parameters should be separated out as a distinct (control or attribute) element.

b. Line spacing and pel spacing should be real values specified as PELS/VDC.

c. I se MMAGE APERATURE serves no useful purpose that cannot be met with CLIP RECTANGLE - it should be removed.

d. There should be a 'local colour precision' parameter for PEL ARRAY. It should also be explicit that not all combinations of compression and l.c.p are legal (e.g., l.c.p must be 1 for CCITT).

e. The document needs more work on colour compression techniques.

f. There is no definition of what a PEL is, and how it differs from a cell or a pixel.

\subsection{TILED PEL ARRAY}

It is not clear that this is not in fact a graphical primitive but a "tiling control function". Better explanation and a name like PEL ARRAY TIING CONTROLS would help.

\subsection{Color Models}

The terminology "Color Model" is commonly used and should replace the terminology "Colour Representation Method" of the Working Draft.

The color models HLS and HSV are missing. There seems to be no good reason to exclude them, particularly since the are in use in the API standards now.

\subsection{User Defined Marker Types}

These are stated in the requirements, and are missing from the Working Draft. It should be evaluated if the need for this is can be satisfied by applying the segment mechanisms of Add.1

\subsection{Additional Hatch Styles}

These are stated in the requirements, but there are none in the Working Draft. The requirements have not been specific on what styles were anticipated. The US simply notes this omission and has no styles to suggest at this time.

\subsection{Interpolated Fill}

This is stated in the requirements, but is missing from the working draft. There are very many ways to do smooth shaded fill. The activity in PHIGS and current practice in the industry should be examined for guidelines for a base proposal or initial set of methods.

\subsection{Transformation of Hatches}

There should be a HATCH TRANSFORM DESIGNATOR element. For engineering practices hatches do not transform, but for other applications they do. There should be a control or attribute element to control this behavior. 


\subsection{References to External Symbols}

This capibility is stated in the requirements, and is missing from the Working Draft. This capability is useful in engineering practice and for "clip art" in graphics arts. The issue of referencing external resources which are not part of or defined by this or another standard is the key issue which needs to be solved in order to include this capability. Once that issue is resolv $d$ it appears promising that the existing segmentation facility of Add.1 can be used to provide a complete mechanism.

\subsection{Improved Text and Font Capability}

Aside from the many specific issues identified below, the US questions the level of font resource information which is being carried in the metafile. The information can be grouped into levels of detail and preciseness. These are based on the font resource subsets of ISO 9541:

- Font Description: resource name itself, family name, design class, weight, posture, etc.

- Font metric information

- Glyph metric and kerning information

- Glyph shape definition.

There should be some amount of the highest level information in the metafile, as part of the font callout procedure. There should be none of the lowest level. How much of the intermediate levels of information should be included is an open issue.

We note that the latest draft of ISO 9541 has dropped the font information subsets concept, which we considered useful information to application commumities.such as computer graphics metafiles.

Following are specific technical topics relating to text.

a. Available glyphs for TEXT primitives. The repertoire of glyphs available with the current CGM character set mechanisms, and means to access glyph collections, is not adequate. A companion standard of ISO 9541 - ISO 10036 establishes the Association for Font Information Interchange (afii) as the registrar for glyphs and glyph collections. Afil estimates that 25000 glyphs will be registered in the next 2 years. It appears that each will have a unique 4 -byte identifier. Add.3 must have a way to access glyph collections that do not fit into the traditional model of "G-sets" and character set switching defined by ISO 2022. At the least a new value of CHARACTER CODING ANNOUNCER is needed. Then the metafile might use afii 4-byte identifiers and/or mapping tables to convert 4 byte ID's to 1 or 2 byte codes.

b. Inclusion and formulation of glyph definitions. There are many technical problems with the formulation of glyph definitions. It is the US position, however, that glyph definitions do not belong in the metafile. There are two reasons:

- Glyph shape descriptions do not belong in the metafile; they should be part of the external font resource, which may accompany the metafile but is not 


\section{a part of it.}

- The work is premature with respect to work on ISO $9541 / 3$, which is just commencing.

Note: the US believes that CGM (Add.3) technology should be used to define an encoding of glyph shape definition, as part of an external font resource, following the functional guidelines established in ISO $9541 / 3$, when work on that part is sufficiently advanced.

c. BOXED TEXT should be dropped and a RESTRICTED TEXT METHOD element added such that the RESTRICTED TEXT element can function in the manner described for BOXED TEXT. The new mode element should standardize at least some text fitting options such as: as-now, text simply is adjusted not to violate box; exact-fit, the CHARACTER HEIGHT is superceded by the box height and the string width is adjusted to exactly fit the box width; width-fit, as previous, but CHARACTER HEIGHT is not altered regardless of its relationship to the box; isotropic-scale, in which the text is scaled without distortion until both dimensions fit and at least one dimension fits exactly; etc. These are just some possibilities. This should be a parameter subject to registration. There may be other desirable values which give more detail about layout algorithms.

d. Access to ISO 9541 font resources. Methods to access all information in 9541 format font resources external to the metafile are needed. Precisely what the practical implications are remains an open issue. The shape description information must be easily accessible. At least one of the technologies used to describe the shapes in the font resource should be compatible with the CGM.

e. Font scoring. The current wording should be clarified to indicate that the score character and the exact score position are defined in the font resource for the current font.

f. Font Terminology. Add.3 terminology should be aligned better with 9541 . 



\section{Attachment 8:}

Draft Addendum Text for CGM Addendum 1 



\title{
CGM Addendum 1
}

\author{
Part 1
}

Draft Copy

August 1989 

Sub-clause 0.1: Add the following at the end of the sub-clause:

This picnure description includes the capability for describing static picnres. Static picnures are those where elements which may lead to dynamic effects (for example those leading to regeneration) are prohibited within the picrure body.

Pogel

Sub-clause 0.3: Add the following at the end of item c):

It should also not preclude funther extensions to support funre standards.

Pagel

Sub-clause 0.3: Add the following at the end of item d):

It should include the capability to support ISO 7942 (GKS) static pictrre-capture.

Poge 3

Sub-claise 0.8: Add the following at the end of the first paragraph:

The CGM specifies the elements required to support ISO 7942 (GKS) static picture-capture.

Pege 3

Sub-clause 0.8: Add the following at the end of the clause:

There is a very close relationship between many of the elements in ISO 8632.1987/ADD.1 and a subset of the finctions in the CGI (Computer Graphics Interiace . ISO DP 9636).

Poge 4

Clause 1: Add the following at the end of the first paragraph:

This picruse description includes the capability for describing static images.

Pages

Clause 2: Add the following to the list of references:

ISO DP 9636 Information processing systems - Computer Graphics - Interfacing techniques for diajogues with graphical devices (CGI). Pars 1.6.

Page 6

Sub-clause 3.1.2.6: Definition of graphical elements

Inser "primitive" between "graphical" and "element".

Puṣéo

Cluse 3: Add the following to the list of definitions and abbreviations:

anisotropic mapping: A mapping in which the scale factors applied along each axis are not equal. This is often used in reference to the mapping from VDC 10 distance units on the physical drawing suriace. See "isoropic mapping". 
boundary: The mathematical locus that defines, in abstract VDC space, the limits of a region to be filled (for fill prinitives and closed figures). The visual appearance of interior style hollow' consists of a depiction of the boundary obtained after elipping has been taken into account. The boundary is distinct from the edge as it includes the intersection of the region with the perimiter of the effective clipping region..

character set: The set of displayaule symbols mapped to individual characters in a TEXT. APPEND TEn $T$. or RESTRICTED TEXT string. This corresponds to the "G-set" defined'in ISO 2022. A character set is independent of the fons or typeiace; examples of character sets: ASCII (X3.4), German Katakann.

clipping mode: A generic term referring to one of Line Clipping. Marker Clipping or Edge Clipping Modes. An object clipping may be either locus', 'shape' or locus then shape'.

closed figure: A compound primitive that behaves as a fill primitive of more general shape. It is formed by bracketing a sequence of line or fill primitives, edge auribures, and cersain controls, with the elements BECIN FIGURE and END FIGURE

compound primitive: A compound primitive is specified by a sequence of CGM elements. as opposed to primitives represented by a single element. Compound text and closed figures are ex amples of compound primitives in the CGM.

compound text: A compound primitive: formed through the use of APPEND TEXT. There may be attribute changes between portions of the resulting complete text string.

device coordinates: The coordinates native to a device; device-dependent coordinates; physical device coordinates.

device viewport: A rectangulas subset of the physical drawing surface into which VDC EXTENT is mapped. See "effecrive viewport".

edge: The rendering of the perimite of a filled region, controlled by edge atributes. Edges are clipped after being applied to the boundary, as distinct from the rendition of the boundary obtained from interior style hollow'. See "boundary".

effective viewport: The acnal viewport resulting from forced isotropic mapping from the VDC extent to the viewpor

foreground colour: The colour used in the rendering process in which primitives are rendered on the drawing surface. as opposed to the BACKGROUND COLOUR or AUXTLIARY COLOUR. The foreground colour is ser separately for each class of primitives.

global segment: A segment that is defined in the Metanile Description (see segment). It may be referenced from withis any picruse

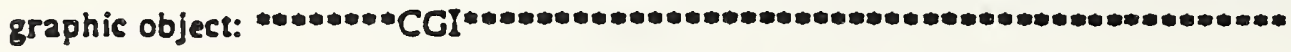

isotropic mapping: A mapping which is invariant with respect to direction: equal scaling in all orthogonal representational dimensions. Often used to describe the mapping from VDC to distance units on the physical drawing surface (see "anisotropic mapping").

local segment: A segment that is local to the picture in which it appears.

region: In the context of closed figures or the POLYCON SET eiement, an area that is explicitly or implicitly closed. that is a subset of the full area being filled. Regions can be nested. disjoint or overlapping. The boundaries of all regions are considered together when applying the interior test for filling a closed figure or POLYCON SET.

segment: A collection of primitives. primitive auributes and some additional attributes associated with the segment as a whole. See "segment atributes".

segment attribute: An attribute associated with a segment as a whole as opposed to attributes of individual primitives.

size spetificution mode: A generic term for Line Width Specification Mode. Edge Width Specification Mode. or .Hiskci size Specification Mode. A size specification mode may be VDC or sealed. the latter being referenced to a numinin sis in device coordinate space. 
skewed: Used to describe stroke precision text when the CHARACTER ORIENTATION vectors are nonperpendicular. CELL ARRAY's when the three derining points form a parallelogram which is not a rectangle. or a segmens transformation thas causes rectangies to become non-restangular parallelograms.

Page9

Sub-clause 4.1: Add the following at the end of the list of classes of elements:

Segment Elements, which enable the grouping and manipulation of elements.

Page 9

Sub-clause 4.1: Add the following after the third peragraph:

Graphical ourput primitives and attributes may be grouped in segments. Segment attribute elements control the appearance of segments.

Page 10

Sub-clause 4.2: Add the following at the end of the sub-clause:

Groups of elements. called segments, are delimited by BEGN SEGMENT and END SEGMENT. Each segment is uniquely identiried by a segment identifier. Segments may be derined in the Metafile Descriptor or within picrue bodies.

Page 10

Sub-clause 4 3: Add the following to the list after the first paragraph:

NAME PRECISION

MAXMMUM VDC EXTENT

SEGMENT PRIORITY EXTENT

NOTE: Other elements, as defined in this standard may appear within the Metafile Descriplor within the definicion of a global segmeni

Page 10

Sub-elause 43: Add the following at the end of the sub-clause:

NOTE: It is recommended that the following elements: METAFILE VERSION, METAFILE ELEMENT LIST and METAFILE DESCRIPTION appear IIrst in the Metafile Descriptor and in the order listed.

Page 10

Sub-clause 43.2: Add the following at the end of the sub-clause:

Furcher shorchands are defined for groups of CGM elements.

Page 11

Sub-clause 43.2: Add the following clauses as the end of the sub-clause:

\subsubsection{Ver.2-static-all set}

The Ver.2-static-all set may be used to indicate all the elements in the drawing-plus-contol set and all the additional elemens defired in ISO 8632/1-1987/.Add.1.

\section{\$3.2.4 Extended-primitives set}


The Extended-primitives set may be used to indicase those primitives which are not derined in ISO 7942 (GKS). These elemenis are:

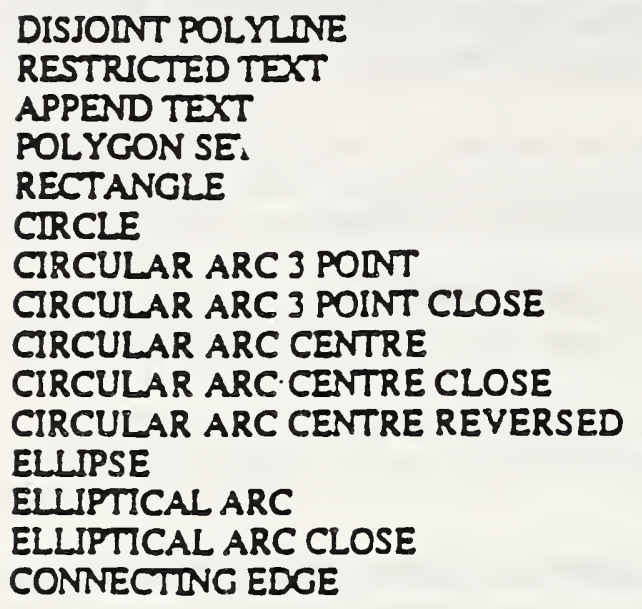

\subsubsection{Ver.2-static-gksm set}

The Ver.2-static-gksm set includes elements for ISO 7942 (GKS) static picnure capnure. The elements included in the Ver.2-static.gkesm set are:

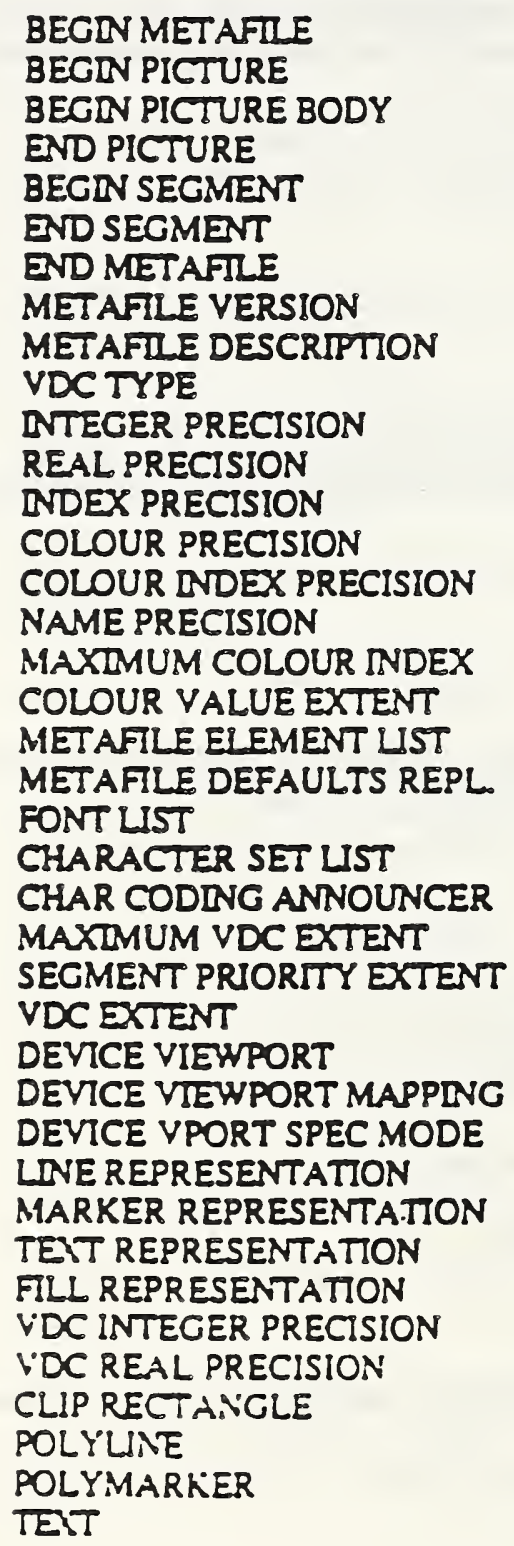


POLYGON

CELL ARRAY

GDP

LINE BUNDLE INDEX

LNE TYPE

LDNE WIOTH

LDNE COLOUR

MARKER BUNDLE INDEX

MARKER TYPE

MARKER SLZE

MARKER COLOUR

TEXT BUNDLE INDEX

TEXT FONT INDEX

TEXT PRECISION

CHAR EXPANSION FACTOR

CHARACTER SPACING

TEXT COLOUR

CHARACTER HEGHT

CHARACTER ORIENTATION

TEXT PATH

TEXT ALIGNMENT

CHARACTER SET INDEX

ALT CHAR SET INDEX

FIL BUNDLE INDEX

INTERIOR STYLE

FILL COLOUR

HATCH INDEX

PATTERN INDEX

FIL REFERENCE POINT

PATTERNTABLE

PATTERN SIZE

COLOUR TABLE

ASPECT SOURCE FLAGS

PICX IDENTLFIER

ESCAPE

MESSAGE

APPLICATION DATA

SEGMENT TRANSFORMATION

SEGMENT HIGHLGHTING

SEGMENT DISPLAY PRIORITY

SEGMENT PICX PRIORITY

Page 12

Add the following text wo the end of sub-clause 4.4.4

MAXIMUM VDC EXTENT derines an extent which bounds the VDC extent values which may be found in the metarile. It may be, bus need not be, a closest bound in the sense that it exactly equals the union of the extent rectangles in the metarile.

Page 14

Add the following sub-clause after sub-clause 4.4.6

\subsubsection{Device viewport control}

The device viewpon specifies the region of the acrual device drawing surface into which the VDC extent is to be mapped on interpretarion. VDC-io-Device mapping is determiniud by the VDC extent device viewport and device viewpor mapping. This type of urassformation is restricted to allow only translation and scaling. No rotation or skew is possible

The position of the device viewport is specificd in one of three unit systems selected by DEVICE VIEWPORT SPECIFICATION MODE elcment: 
- by fraction $[0.0-1.0]$ of the available drawing surface, which allows ressonable placement and relative sizing of the viewpors:

- in millineres tines a scale factor. which allows absolute sizins of images:

- in physical device units.

The DEVICE VIEWPORT MAPPING element may be used to force isotropic mapping even if the specified VDC extent and device viewpor would not otherwise have led wo one In such a case. the VDC extent is mapped onto a subset of the specified device viewpors on interpretation. This subset is defined by shrinking either the vertical or horizontal dimension of the specified viewport as needed wo reach the required aspect ratio. This smallex "effective viewport" is then used to derine the coordinate mapping from VDC to the device's coordinates. The placement of the effective viewport rectangle within the original one can be specified. This placement can be one of left, right or centred when the shrinking is horizontal, and top. bottom or centred when it is vertical. 'Left' and 'bottom' are interpreted as being wowds the 'Iust come' of the specified DEVICE VIEWPORT regardless of any mirroring or rotation of the vieu port on the physical device. These meanings are relative to the recengle defined by the non-inverted viewport.

For VDC Extent the coordinates can increase or decrease from the first to the second corners. If decreasing coordinates are chosen, this will lead to mirroring or rotation of primitives.

The behaviour of primitives and geometric atribures under transformations is further described in sub-clause 4.6.

If both device viewport and scaling mode appear in the same mesarile then the last specified is used. If neither appear then the defauls values for device viewpor lake procedence.

\subsubsection{Representations}

The elemenLS LINE REPRESENTATION. MARKER REPRESENTATION, TEXT REPRESENTATION, FILL REPRESENTATION and EDGE REPRESENTATION are used to ses all of the auribute values in a bundle table critry as the same ime. The astributes. which may be bundied, are described in 4.7.

Page 14

Add the following at the end of sub-clause 4.5:

Some of the control eiements may appear in the Picrure Descriptor if this is permitted by the formal grammar for the metarile version.

Page 15

Add the following text to the end of sub-clause 4.5 .2 .

There are three different clipping modes for lines, markers and edges. The required clipping mode is recorded in the metanile with the elements: LINE CLIPPING MODE, EDGE CLIPPING MODE, and MARKER CLIPPING MODE. When the CLIP INDICATOR associared with a graphical primitive is 'on', only those parts of a graphical primitive that are considered inside the effective clipping region are rendered on interpretation. The objoct clipping modes allow precise specification as to how clipping is applied to primitives on interpreation.

Clipping may be either locus'. 'shape' or 'locus then shape'. Conceptually, a locos is a mathematical object like a point or line segment, while a shape is an area in 2-dimensional space. Loci are 0-1- or 2-dimensional subsets of real-valued 2.space. For markers and text they are points. For lines they are the individunl line segments or portions of ares. The locus of an area is the shape and the boundary. Shapes reflect the reviization of geomerrie auributes and are generally 2. dimensional subsets of real-v alued space

'Locus' elipping is applicd for each portion of a graphic object based on its mathematical location and is independent of the area it will iceupy after rendering. For example no portion of a line segment is rendered if the ideal mathematical line lies outside the effective clipping region. (even if its line width would carry some portion of the rendering of it into the clipping :ectangle): no portion of a marker is rendered if its location lies outside the clipping rectangle.

If "Locus' c!iping is uscd. the rendering is applical to the locus of the graphic object. The resulting rendered shape areas may: therefore cxtersd oulside the effective clipping region. 
'Shape' clipping is applied after the abstract rendering of shape in device coordinate space, the 2-dimensional point set associated with the graphic object is intersected with the effective clipping region, which has been transformed in device coordinate space.

'Locus then shape' clipping allows the specification that both locus and shape clipping be applied to graphic objects as described above. In this case however. the rendered shape will not extend outside the effective clipping region. A thick line whose locus is outside the clip window will not have any portion visible even if its line width would carry some porion of the rendering outside the clip rectangle.

Figure *tonen shows some examples of the effect of the clipping modes.

When a wideh or size specification mode is 'sealed', the rendering of shape proceeds in DC space after application of the VDC-to-Device mapping.

Fill and text primitives do not have associated object clipping modes. (though the edge of a fill primitive and the boundary edges of a closed figure do). Clipping for flll primitives is always consistent with shape clipping (see subclause 4.6.4.5). For text primitives, the type of clipping is determined by the associated text precision:

For 'string' precision text clipping proceeds, on a per string basis, in a manner consistent with 'locus' clipping.

For 'character' precision texh, clipping proceeds, on a per character basis. in a manner consistent with locus elipping.

For 'stroke' precision text the clipping always proceeds in a manner consistent with shape clipping.

Note that shape clipping for all text precisions is always allowed by this Standard.

Clip rectangles applied to graphical primitive elements within segments may be subject to transformations in VDC space. Irressection of clip receangles (untransformed or transformed) may lead to resulting polygonal clipping boundaries (see 4.12.5).

Pege 15

Add the following to the list of graphical primitive elements and to the list of line elements in sub-clause 4.6

\section{CIRCULAR ARC CENTRE REVERSED}

CONNECTING EDGE

Page 16

Add the following before sub-cluse 4.6.1:

In addition to the graphical primitive elements listed above, this Standard defines elements providing the definition of 'compound primixives' from several of the othes graphical primitives. The following classes of compound primitives are definedi 'compound lext' and 'closed figures'. The elements thas may be used 10 specify compound primitives are listed in Table *omosecose. .

Table *.*s* Compound Primitives

\begin{tabular}{|c|c|c|c|c|}
\hline $\begin{array}{l}\text { Objeat } \\
\text { Class }\end{array}$ & $\begin{array}{l}\text { Fust } \\
\text { Elemens }\end{array}$ & $\begin{array}{l}\text { Primitives } \\
\text { Inctudad }\end{array}$ & $\begin{array}{l}\text { Other } \\
\text { Elements }\end{array}$ & $\begin{array}{l}\text { Final } \\
\text { Element }\end{array}$ \\
\hline $\begin{array}{l}\text { Compound } \\
\text { Text }\end{array}$ & $\begin{array}{l}\text { TEXT } \\
\text { RESTRICTED } \\
\text { TEXT (NoLE 1) }\end{array}$ & $\begin{array}{l}\text { APPEND TEXT } \\
\text { (Note2) } \\
\text { GDP (Nole 5) }\end{array}$ & & $\begin{array}{l}\text { APPEND TEXT } \\
\text { Note 3) } \\
\text { GDP (Note 5) }\end{array}$ \\
\hline $\begin{array}{l}\text { Closed } \\
\text { Figure }\end{array}$ & $\begin{array}{l}\text { BEGIN } \\
\text { FGURE }\end{array}$ & $\begin{array}{l}\text { Line Primitives } \\
\text { Fill Primitives } \\
\text { (Note 4) } \\
\text { GDP (Note S) }\end{array}$ & $\begin{array}{l}\text { NEW } \\
\text { REGION }\end{array}$ & $\begin{array}{l}\text { END } \\
\text { FGUURE }\end{array}$ \\
\hline
\end{tabular}

Notes: 
The final/not final flag is 'not final; the primitive defines the reference point of the entire compound text prinitive; the text of the primitive is entered in the buffer.

The finai/rot final tas is not firal."

The finalinot final flag is 'not final'; the $2 \times 1$ of the primitive is entered in the buffer before the compound primitive is closed.

All primitives of the identified classes may be included.

Whecher a GDP may contribute to compound text or closed figures. and whether or how it specinies that the compound lext state or elosed figure state be opened, maintained or closed, is specified with the definition of the GDP in the Intenational Register of Graphical Items.

Graphical primitive elements and compound primitive elements may be subject to tansformation in VDC space (segment and copy transformation, 4.124 .2 and 4.125). Such a transformation may change the shape of some primitives. If there is a skew, a primitive initially specified as a rectangle may become a paralelogram. If there is an anisotropic scaling, a primitive initially specified as a circle may become an ellipse. Note that the shape of markers is exempt from such ransformations.

Page 16

Sub-clause 4.6.1.1 add the following text to the paragraph descibing CIRCULAR ARC $x \times x$

A reverse direcion are can also be specified, this is descibed in $\mathbf{5 . 6 . 2 0}$

Page 16

Add the following at the end of sub-clause 4.6.1.1

CONNECTING EDGE A line segment comecting the last point of the preceding line element to the next point is generuted during the construction of a closed figure. The next point is either the first point of the next line element or the currens elosure poins

Page 16

Add the following at the and of sub-clause 4.6.1.3

In version two meandes line clipping is controlled by the LINE CLIPPING MODE element which can have one of the following values: Tocus', 'shape', or 'locus then shape'. However. clipping only applies if the CLIP INDICATOR is 'on'.

For 'locus' elipping, the mathematical locus of the line is clipped at the intersection with the clip rectangle before shape rendering is applied Hence part of the shape of a line may appear outside the clip rectangle.

For 'shape' elipping, the shape of the rendered line is clipped to the intersection with the clip recungle that is nothing is dawn ourside the clip rectingie.

For locus then shape' clipping, the mathematical locus of the line is clipped, as with locus clipping, and then subsequently the rendered shape of the clipped locus is again clipped. Note that since the mathematical locus of the line may have changes as a result of locus clipping. subsequent shape rendering and clipping may produce a different appearance of a line from either of the other two clipping modes.

If the line width is measured in VDC units it is subject to VDC-to-Device mapping (4.4.7) as well as to both segment and copy transformation (4.124.5 and 4.12.5). Note that the entire locus of an are is subject to these transformations. In case of an anisotropic mapping or transformation the rendered width of the line will change with the direction of the line segment. If the line wideh is speciried as a scale factor it is not affected by any transformations.

Page 17

Sub-clause 4.6.2.3: Add the following at the beginning of the sub-clause:

The following discussion applies wo vesion 1 melafiles. 
Sub-clause 4.6.23: at the end of the first paragraph change 'is not standardized.' to the following:

is not sundardized for version 1 meuriles.

Pege 17

Sub-clause 4.6.23: Add the following at the end of the sub-clause:

In version 2 metariles, marker clipping is controlled by the MARKER CLIPPING MODE element which can have one of the following values: locus', 'shape' or locus then shape'. However. elipping applies only if the CLIP INDICATOR is 'or'.

For locus' clipping, the mathemarical locus of the markers (that is the specifying points), are clipped at the intersection with the clip rectangle before shape rendering is applied. Hence, part of the shape of a marker may appear outside the elip rectangle. However the marker is visible if, and only if, its specifying point is within the clip rectangle.

For 'shape' clipping, the shape of the rendered marker symbols are clipped to the intersection with the clip rectangie. that is nothing is drawn outside the elip rectangle. Portions of the marker symbol may appear inside the clip rectangle even though the marker's locus is ourside.

For locus then shape clipping. the mathematical locus of the markers are clipped. as with locus clipping, and then subsequently the rendered shape of the maxkers are again clipped.

If the marke size is measured in VDC units. it is subject to VDC-w-Device mapping (4.4.7) as well as to both segment and copy transformation (4.12.45 and 4.125). The shape of markers is never affected by transformations. for exampie a eirele used as a marker type shall always appear as a curcle. Onily the marker size may be transformed. For this purpose. conceparally, vectors with the iength marker size and arbitray orientations are transformed: the resulting marker size is determined by the orientation of the vector which maximizes the length under the transformation (euclidean norm of $2 \times 2$ tounsformacion marix).

If the marke size is specifled as a seale factor it is not affected by any transformations.

Page 18

Add the following at the end of sub-clause 4.6 .3 .3

Clipping of text strings is described in 4.7.6.

The vectors speciñed by the CHARACTER ORIENTATION element (4.7.6) are subject to the VDC-Lo-Device mapping as well as to both segment and copy transfomation.

Page 19

Add the following as the end of sub-clause 4.6 .45

Edge clipping is controlled by the EDGE CLIPPDNG MODE element, which has the same enumerations as LDNE CLIPPING MODE. Edges are clipped in the same way that lines are clipped see 4.6.1.3

Page 19

Add the following sub-clause after sub-clause 4.6.45:

\subsubsection{Transformation}

The entire locus of rectangles. circular and elliptical filled-area elements is subjecs to VDC-to-Device mapping (4.4.7). segment and copy transformations (4.12.4.5 and 4.12.5). These elements may not therefore retain their specificd geomery afier transformation.

The veciors of the PATTERN SLEE element are subject to all transformasions.

The edge widths are treated in exacty the same way as line widths (4.6.1.3). 
Add the following sub-clause afrer sub-clause 4.6.7

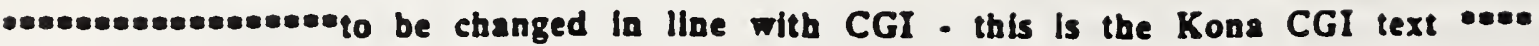

\subsubsection{Closed ngures}

3.9.5.1 Construction of closed figures. A closed figure is a fill type compound graphic object which the. client constructs on the device side of the interface by invoking BEGIN FIGURE, an ordered sequence of line and fill primitives (and optionally attributes and NEW REGION functions), and END FIGURE. Edge attribute values are associated locally with the edge portions, and fill attribute values associated globally with the complete graphic object: in addition, cenain general attribute values are associated locally with edge portions and globally for the interior of the fill object. The entire graphic object then travels through the CGl pipeline, and is rendered as a single unit with the parity fill algorithm described elsewhere.

3.9.5.1.1 Closure Point. The first point of the first line primitive in a new region is the closure point for that region. The Virtual Device retains this closure point for use in closing the region. When the region is closed (with a NEW REGION or END FIGURE function, or by invoking a fill primitive which begins a new region), a line segment from the last point of the last line primitive in the region to this closure point is added by the Virtual Device to the figure, uniess these points are already coincident.

3.9.5.1.2 Regions. The closed figure consists of one or more regions; a region has a closed boundary which may be concave, convex, and self crossing. A region is formed either by invoking a fill type primitive in FIGURE OPEN state (which closes the last region and contributes one or more complete regions), by invoking NEW REGION to start new regions to be formed from line primitives, or by a final invocation of END FIGURE. A closed figure constructed from only line primitives without use of NEW REGION consists of a single region.

The NEW REGION function may appear anywhere in the closed figure. If the current region is closed, the function is ignored. If the current region is open, an implicit boundary portion is added from the last point of the last primitive to the current closure point unless CONNECTING EDGE has been invoked after the last line primitive, in which case an explicit boundary portion and edge portion is added instead.

3.9.5.2 Boundaries and Edges. Each region consists of a combination of implicit boundary portions and edge portions.

3.9.5.2.1 Explicit. Explicit boundary portions and edge portions are those added by client invocation of primitives in state FIGURE OPEN. These

are generated in the following situations:

- for fill primitives other than POLYGON SET, the complete edge becomes an explicit boundary portion and edge portion in the closed figure.

- for line primitives. those portions which would be rendered outside of state FIGURE OPEN become explicit boundary portions and edge portions. 
- For DISUOINT POLYLINE, in particular, only the segments from the first point to the second point, from the third point to the fourth point, and so on, become explicit boundary portions and edge portions when incorporated into closed figures.

- A CONNECTING EDGE primitive which precedes an action which would normally have added an implicit buundary portion to the figure either to close a region (including closing the figure itseli) or to connect two line primitives results in the portion added being an explicit boundary portion and edge portion. CONNECTING EDGE preceding of following DISJOINT POLYLINE or POLYGON SET does not affect the interpretation of those functions with respect to boundaries and edges.

Edge portions take associated edge attribute values from the state list; as these state list entries can be changed between the primitives that result in edge portions in FIGURE OPEN state, each edge portion has a distinct set of attribute values associated with it.

3.9.5.2.2 Implicit. Edge. attributes are never associated with implicit boundary portions. Implicit boundary portions are only rendered for interior style hollow and are a special representation of the interior, not a representation of any portion of the edge.

Implicit boundary portions are added by the CGI device to the figure definition under the following circumstances:

- invocation of NEW REGION, END FIGURE, or a fill primitive when the current region has not been explicitly closed and CONNECTING EDGE has not been invoked since the last line primitive: an implicit boundary portion is added from the last point of the last primitive to the current closure point to close the region.

- when the last point of the preceding line primitive is not coincident with the first point of the current line primitive, an implicit boundary portion is created to connect the last point of the preceding line primitive to the first point of the current line primitive.

- the portions of a DISJOINT POLYLINE whict would not normally be rendered (i.e., from the second point to the third point, from the fourth point to the fifth point, and so on) result in implicit boundary portions. (These are additional to the ones which may be added to connect to a preceding or following line primitive or to effect region closure after the DISJOINT POLYUNE)

- the portions of polygon set as described below.

3.9.5.2.3 Conditions under which no boundary or edge is added. No boundary or edge portion is ever created connecting two regions, regardless. of how those regions were created or closed.

\subsubsection{Contribution of Primitive Functions to the Figure.}

3.9.5.3.1 Contribution of Line Functions to the Figure [continue with what was 3.9.5.1.1 Add this before final paragraph of the section:]

\section{CONNECTINGEDGE}

If the region is open. the start point of the connecting edge is the last point of the last line primitive, and the end point of the connecting edge is either the first point of the following primitive or the current closure point as described above. If the connecting edge would be of zero length (i.e., if the two points it connects are coincident), the function is ignored. As 
with other line primitives, the edge attribute values in effect at the time it is invoked are associated with any edge portion generated by this function.

If the current region is not open, invocations of the CONNECTING EDGE function are ignored (i.e., CONNECTING EDGE cannot be used to connect regions).

CONNECTING EDGE is a primitive, not a modal setting: it must be invoked once for each connecting edge desired in the figure, and once used, no longer applies to subsequent opportunities for explicit connection.

Invoking CONNECTING EDGE multiple times after a line primitive results in the last instance (with its associated attributes) being used.

[continue with last paragraph of section]

3.9.5.3.2 Contribution of Fill Functions to the Figure. [replaces 3.9.5.1.2] Each fill primitive contributes a complete region to the figure (POLYGON SET may contribute more than one). after first closing the current region if it is open. The CGl device periorms an implicit NEW REGION before and after a fill primitive invoked in FIGURE OPEN state (i.e., a fill primitive leaves the current region closed, and the next primitive begins a new region).

The unclipead boundary of each fill primitive contributes to the unclipped boundary of the closed figu: :; the locus of its interior does not affect the boundary definition.

Contribution of POLYGON SET to figure construction:

- A POLYGON SET is considered to contribute one or more complete regions. If the current region has not been closed, an implicit NEW REGION is performed before the POLYGON SET is added to the figure definition. If the POLYGON SET does not end with a point whose edge-out flag is "elose visible" or "close invisible" an implicit NEW REGION is performed after the POLYGONSET.

- Sequences of points "with edge-out flag "visible" are treated as if they were polylines, terminating with the first point with a different edge-out flag. Each such polyline becomes an edge portion of the boundary of the figure. The edge attribute values in effect when POLYGON SET is imvoked are associated with any edge portion added in this way.

- Sequences of points with edge-out flag "invisible" contribute implicit boundary portions which are polylines joining the points in the sequence, but not edges. Edge attribute values are not associated with these.

- Points with edge-out flag "close invisible" generate the equivalent of a NEW REGION, generating an implicit boundary portion from this point to the current closure point if these are not coincident, and closing the current region.

- Points with edge-out flag "close visible" generate the equivalent of a CONNECTING EDGE followed by a NEW REGION, resulting in an edge portion from this point to the current closure point if these are not coincident. The edge attribute values in effect wtien POLYGON SET is invoked are associaled with any edge portion added in this way.

- POLYGON SET does not affect the value of the edge visibility value in the state list.

3.9.5.3.3 GDP. A GDP which is defined as a line type primitive must specify which is the first point and the last point in its point list, with respect to closed figure construction. 
Such GDP's are assumed to contribute to a closed figure a boundary corresponding to thie unclipped locus which would be rendered if the function were invoked when not in FIGURE OPEN state; any other behaviour shall be documented explicitly in the GDP description. A GDP which is defined as being a fill type primitive function is treated as in the previous section; any variation or special handling in state FIGURE OPEN shall be explicitly documented in the GDP description.

\subsubsection{4 [replace 3.9.5.1.4-6] Association of attributes.}

3.9.5.4.1 Local attributes are those associated with each edge portion, which can vary from edge portion to edge portion within the compound object. The local attributes for closed figures are the set of edge attributes.

3.9.5.4.2 Global attributes are those associated with the compound object as a whole pather than its component parts. The functions which set their state list values may be invoked during FIGURE OPEN state, and have their usual effect on the corresponding state list entries. The values associated with the closed figure are those in the state list when END FIGURE is invoked to complete the object formation (even in the event of figure buffer overflow during construction). Global attributes of closed figures are the set of fill attributes, CLIP. INDICATOR, CLIP RECTANGLE, EDGE CLIPPING MODE, and PICK ID. In particular, note that Clip Rectangle and Clip Indicator are associated with the compound object, but not applied during graphic object formation (the graphic object is formed from the unclipped locus of each primitive function invoked in FIGURE OPEN state).

3.9.5.4.3 There is a set of attributes which are local attributes with respect to the edge portions, but which are associated globally with the interior. This set consists of AUXILIARY COLOUR (and its corresponding colour selection mode in which set). TRANSPARENCY, and DRAWING MODE. In order to use a different value for the interior from that for any of the edge portions, the appropriate attribute function should be invoked just prior to invoking END FIGURE with the value to be used for the interior.

-...........end CGI text

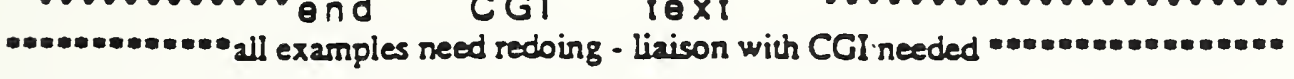

4.6.8.8. Examples

Note: The elear text encoding is used here for illustration purpose.

\footnotetext{
******need updating

Example 1.
} 
This example uses the Are Centre command to create a doughnur shape. The following commands are used:

\section{Beginfig:}

AreC $\square$ 50.50 1.0 1.0 45;

AseC $50,501.01 .040$;

EndFig:

Note that this figure can also be obtained by the sequence:

Begin Fig:

Circle 50.50 45:

Circle 50.50 40;

EndFig; 
This cxample uses the Elliptical Are command wo creake a box with rounded comers. The following commands are used:

\section{Beginfig:}

\% All straight edges connecting the elliptical ares \%

$\%$ are drawn as implicit edges \%

EllipArc 75,82 90,82 75.110 1.0 0.1;

EllipAre $25.8225 .11010 .820 .1-1.0$ :

EllipAre 25.38 10.38 25.10 - 1.0 0.-1:

EllipAre 75,38 75,1090,38 0,-1 1,0;

EndFig:

\section{Example 3.}

This example uses the Eliptical Arc command, showing how CDP order can be used to change the sweep direction. The lines indicate the shor angles berween the CDP's. The following commands are used:

\section{Beginfig:}

$\%$ All straight edges connecting the clliptical arcs \%

90 are drawn as implicit codges $\%$

\%. The first are is swept in a counterclockwise direction \%

EllipAsc $60.5060 .100 \cdot 10.500 .10 .1$ :

qc The second are is swept in a clockwise direction \%

EllipArc 60.5060 .100 .500 .10 .1 : 
Add the following sub-clauses after suber suse 4.7.8

\subsubsection{PIck Identifler}

The Pick Identifier is associaled with graphical primitive elements within segmenis (see clause 4.12). It is the only acribute element which does not affect the appermance of a graphical primitive element. It merely establishes a means of identification of primitives within segments at metafile interpretation. PICK IDENTIFIER has no graphical effect and is av ailable for application dependent communication berween interpreters and generators.

\subsubsection{Global and local attributes and controls}

For the purpose of compound primitive definition (see 4.6) a further classification of attributes and control ciements into "global" and 'local' attribute and control elements is introduced. Global elements apply to compound primitives as a whole, while local elements apply separatly to the component graphical primitives of a compound primitive.

Page 40

Add the following sub-clauses after sub-clause 4.11:

\subsection{Segment elements}

\subsubsection{Introduction}

In the CGM. graphical primitive elements or compound primitives, attribute setting elements and certain control elements may be grouped in segments as well as being invoked outside segments. They may also be derined as global segments, within the Metafile Descripror, and can then be copied into a picaure. Each segment is identified by a unique segment identifier. Segments may have the atuributes:

2. transformation:

b. highlighting:

c. display and pick priority;

These may be defined at segment definition time, before the first primitives of the segment and shall not be changed thereafier for suacic picture-capare metarles.

Only elements stored inside segments are affected by the segment atributes.

The segment elements are:

COPY SEGMENT
INHERTANCE FILTER
CLIP INHERTANCE
SEGMENT TRANSFORMATION
SEGMENT HIGHLIGHTING
SEGMENT DISPLAY PRIORTY
SEGMENT PICK PRIORITY

BEGIN SEGMENT and END SEGMENT are delimiver elements rather than segment elements.

\subsubsection{Global and local segments}

There are two types of segments: local segments and global segments. Both contain primitives and atributes which can be manipulated in the manner described above. Local Segments have no existence beyond the bounds of the picture body in which they are defined. Defining a local segment in a picture automacicaliy includes that segment in the picrure's umage. In contrast. global segments can be referenced by any of the pictures in the metarile in which they are defined. 
A global segment is delimited by the BEGIN SEGMENT and END SEGMENT elements. Global segments are defined in the Metafile Descriptor. They are nor a part of any picoure within the metafile. They must be accessed from within individual pictures by the COPY SEGMENT (4.12.5) element. The COPY SEGMENT element incorporates the segment into the open picnure in the : wne way for both local and global segments.

\subsubsection{Allowable elements In MD and GSS states}

BEGDN SEGMENT is the only segment-related element that is allowed within the Metafile Descriptor State (MDS) (see Table 3(a), the Metaile Sute Table). BEGIN SEGMENT changes the state to Global Segment State (GSS).

\subsubsection{References to global segments}

Within pictures. no elements are allowed that would modify the contents or default appearance of global segments (see Table 3(a)). This restriction preserves the logical independence of pictures and the ability to randomly access pictures. The only allowable references to global segments within pictures are by using the COPY SEGMENT elemenL

\subsubsection{Association of control and attribute elements and attribute elements with primitlves Inside segments}

The current modal values of control and atribute elements are associated with the primitives inside local segments. The modal values established by seuing control or atribute clements within a segment remain outside the segment until they are explicitly changed.

Control and atribute elements are bound in global segments as they are in local segments. Upon the occurrence of BEGIN METAFILE. every element that is modally defined and bound to primitives (Metafile Descripror elements defining modes and precisions. Pienure Descriptor elements. Control elements Aturibute elements and Segment Control elements) has a default value. Conceprually the set of all of these define a "Modal Stare List".

The Metafile Descriptor is processed sequentially. Throughout the Metafile Descriptor, modal MD elements modify the MD entries in the state list and occursences (possibly multiple) of the METAFILE DEFAULTS REPLACEMENT element allow manipulation (outside of GSS state) of the rest of the modal elements (as well as explicitly changing the defaults). Within GSS state the allowable modal elements (control. aturibute, and segment aturibute) also alter the conients of the Modal State List. The values of modal elements that are in effoct upon BEGIN PICTURE are the defauls values for thas picure, whether they are implicis (defined in the Standard) or explicit (that is by values set in the Metafile Defaults Replacemens).

\subsubsection{Dellmiting and naming segments}

The contents of a segment are delimited by the elemenis BEGIN SEGMENT and END SEGMENT. The elements in between these two delimilers are a par of that segment. Each segment has an identifier associated with it. No two global segments may have the same identifier and no local segment may have an identifier which is the same as either a local segment in the same picture or the same as a global segment.

\subsubsection{Segment attributes}

\subsubsection{Introduction}

The segment atributes associated with each segment control its' display. Segment attributes can be set only while the segment is open. These may be defined at segment definition time, only before the first primitives of the segment, and may not be changed thereafter. When a segment is opened with the BEGIN SEGMENT element, the segmeni's atributes are set to their default values. Segment atributes. if set, shall be set immediately after the BEGIN SEGMENT element and before any other type of element. This structure is shown below.

BEGIN SEGMENT (Segment idcnuificr)

Segment auributes

Allowed primitives, atributes and control clements in any order

- END SEGMENT

\subsubsection{Segment transformation}


The segment transformation is a coordinate transformation associated with each segment and applies to all graphical primitives in the identified segment and will be used on interpretation. Clipping rectangles are not transformed by the segment transformation. It allows sealing, transiation, and rotation of segments wo be defined during segment definition.

The segment transformation is a transformation of VDC space to VDC space and is distinct from the VDC-to.Device mapping which is a transformation of VDC space to device coordinates.

The transformation atribute of a segment may be defined by the SEGMENT TRANSFORMATION element curring the segmens definicion A segment transformation is represented by a $2 \times 3$ matrix, composed of a $2 \times 2$ sealing and rotation portion, and a $2 \times 1$ translation portion. The defaule segment transformation is represented by the identity transformation. If the SEGMENT TRANSFORMATION element is not stored in the metanle then all coordinate data is mapped using oniy the VDC-w.Device mapping. If the SEGMENT TRANSFORMATION is.stored in the metafile, it is applied before the application of the VDC-to-Device mapping.

The use of segment transformations may produce coordinates that cannot be expressed within the VDC range. This is hendled in an interpretation dependent way.

\subsubsection{Segment blghllghting}

Segment highlighting can take one of two values. NORMAL and HIGHLIGHTED. The setting of this attribute selects one of these two states for the segment.

\subsubsection{Segmeat display priority}

The display priority atribute of a segment determises how overlapping segments are displayed. Segments with higher display priorities will be dispiayed as if they were in front of segments with lower display priorities. The segment display priority may be normalized to the continuous range of real numbers. zero to one, by applying the minimum exient and maximum extent values provided by the Metafile Descriptor element SEGMENT PRIORITY EXTENT. Interpresution of SEGMENT PICK PRIORITY has no graphical effect. Its generation and interpretation are implementation dependent

\subsubsection{5 ${ }^{\circ}$ Segment pick priority}

The pick priority atribute of a segment is used to resolve the picking of segments which overlap. The segment pick priority may be normalized to the continuous range of real numbers. zero to one. by applying the minimum extens and maximur extent values provided by the Metrile Descriptor element SEGMENT PRIORITY EXTENT.

\subsubsection{Copy segment and inheritance}

The COPY SEGMENT insers the elements of the referenced segment into the picture at the point of occurrence of the element

The elements copied may be altered in a veriery of ways:

2 The inheritance filte mechanism controis whether individual atoribute values are reapplied to the elements

b The clip inheritance mechanism controls whether the primixives in the segment are clipped to the current clip recengle or to a combination of the current and the segment clipping rectangles.

c. The primitive elements are transformed by the copy segment transformation and optionally by the segment transformation of the copied segment according to the rules for transformation

COPY SEGMENT has a transformation matrix as a parameter. The copy segment transformation is applied to graphical primitives before they are copied. This also applies to clipping rectangles in the segment (see below). Gaphical primicives may be transformed to alter their location size. and oricntation.

A segmens may be referenced by the COPY SEGMENT element. either within a picrure or in a global segment. The atrributes associated on interpresation can be those bound to the segment being copied, or can be imposed by the inclusion of the INHERITANCE FILTER element.

The clipping associated with a segment can be that associated with the picture at the time of the copy or can be a combination of the current clipping and the segment clipping when the CLIP INHERITANCE element is used. 
The inheriunce filter mechanism allows the use of the current values of atributes and controls to be associuted with the copied segmens in place of the artributes and controls bound to the primitives when the segment was created. The atribules and controis to be associated with the segment can be all atributes or can be a subset of atrributes. The atributes and controls are selected using the INHERTTANCE FILTER element. The atributes and controls can be selected using individual or group names for atributes. controls and ASFs. The elements yhich can be selected are shown in Table *a for atuributes and controls and in Table *** for ASFs.

The individual element rames as well as the group names are those in the table showing atribute groups below.

If an arribute or group of atributes designated in the filter selection list is set 10 'state_list'. graphics objects inherit that atribute or group of awibutes from the current modal values when a segment is copied

If an atribute or group of attributes designated in the filter selection list is ser to 'segment', that atribute or group of auribures is unaffected (in all graphics objects employing them) by the corresponding current state list when a segment is copied.

The default inheritance filter seuing value is 'segment' for all atuributes and controls.

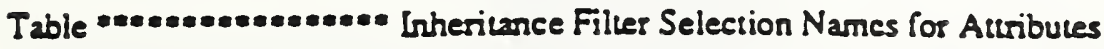

Attribute Group Name

LDNE ATTRLBUTES

MARKER ATTREUTES

TEXT ATTRIBUTES

CHARACTER ATTRIBUTES

FILL ATTRIBUTES

EDGE ATTRIBUTES

PATTERN ATTRIBUTES

OUTPUT CONTROL

PICK IDENTIFIER

AU ATIRIBUTES

ALL
Individual Attribute Name

LINE BUNDLE INDEX

LDNE TYPE

LDE WIDTH

LNE COLOUR

LDVE CLIPPING MODE

MARKER BUNDLE INDEX

MARKER TYPE

MARKER SLEE

MARKER COLOUR

MARKER CLIPPDNG MODE

TEXT BUNDLE INDEX

TEXT FONT INDEX

TEXT PRECISION

CHARACTER EXPANSION FACTOR

CHARACTER SPACING

TEXT COLOUR

CHARACTER HEIGHT

CHARACTER ORIENTATION

TEXT PATH

TEXT ALIGNMENT

FIL BUNDLE DNEX

DNTERIOR STYLE

FILL COLOUR

HATCH INDEX

PATTERN DNDEX

EDGE BUNDLE DNDEX

EDCE TYPE

EDGE WIDTH

EDGE COLOUR

EDGE VISIBIITY

EDGE CLIPPING MODE

FILL REFERENCE POINT

PATTERN SLEE

AUXILIARY COLOUR

TRANSPARENCY

PICK IDENTIFIER

All atributes

All atcributes and control elements 
Table XCXX Inheritance Filier Selecrion Names for Aspect Source Flags

ASF Group Name

LDVE ASFS

MARKER ASFS

TEXT ASFS

FILL ASFS

EDGE ASFS

ALL ASFS
Individual ASF Name

LNE TYPE ASF

LINE WIDTH ASF

LINE COLOUR ASF

MARKER TYPE ASF

MARKER SLEE ASF

MARKER COLOUR ASF

TEXT FONT DNDEX ASF

TEXT PRECISION ASF

CHARACTER EXPANSION FACTOR ASF

CHARACTER SPACING ASF

TEXT COLOUR ASF

DNTERIOR STYLE ASF

FILL COLOUR ASF

HATCH INDEX ASF

PATTERN INDEX ASF

EDGE TYPE ASF

EDCE WTDTH ASF

EDGE COLOUR ASF

All aspect source flags

Ar example of the COPY SEGMENT element with the INHERTTANCE FILTER element is as follows:

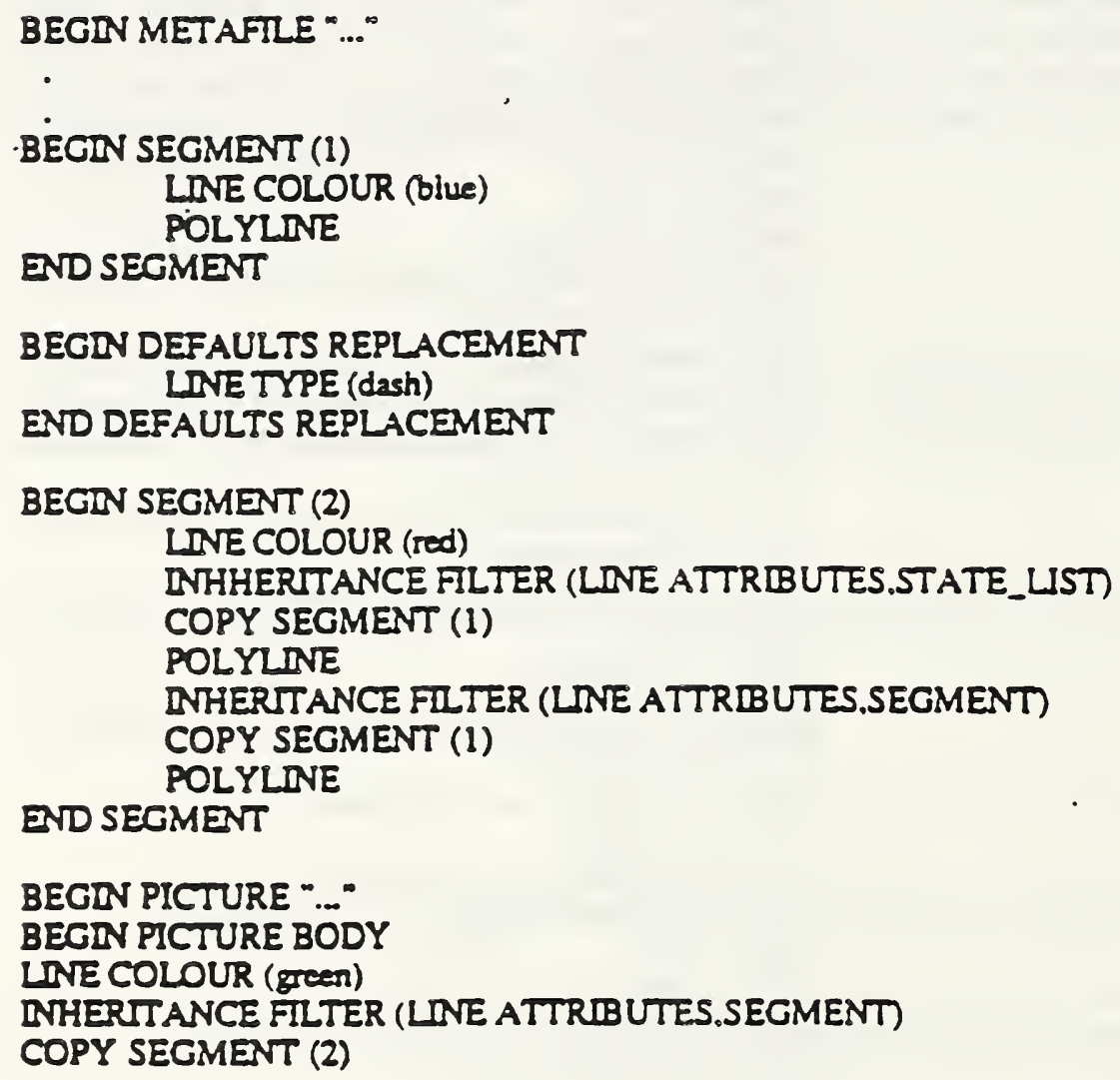




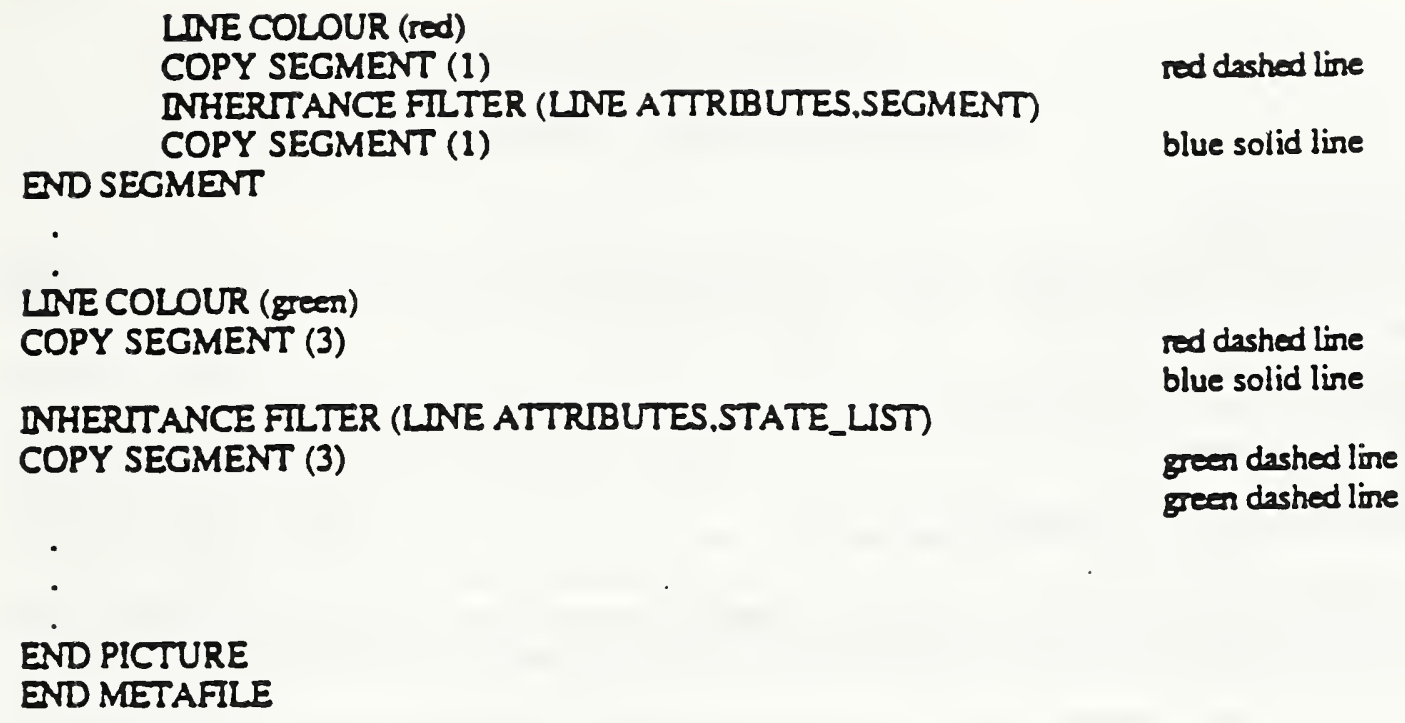

red dashed line

blue solid line

Clipping is not included in the DNHERTTANCE FILTER. There is a separate clement that controls clipping behaviour CLIP INHERTTANCE. Its values may be eicher 'state_list' or 'intersection'.

If the value is 'state_list', then the clip rectangle associated with primitives in the copied segment is that of the last CLIP RECTANGLE encountered in the metarile element sequence prior to the COPY SEGMENT elcment, that is the value in the "modal state list".

If the value is 'intersection', and if both the modal state list clip indicator of the segment are 'on'. then the intersection of the copied segment is the intersection of the modal state list rectangle and the primitive's associated clip rectangle. If either indicator is 'off. then there is no contribution from its associated rectangle. To illustrate; if TA is the copy tursformasion:

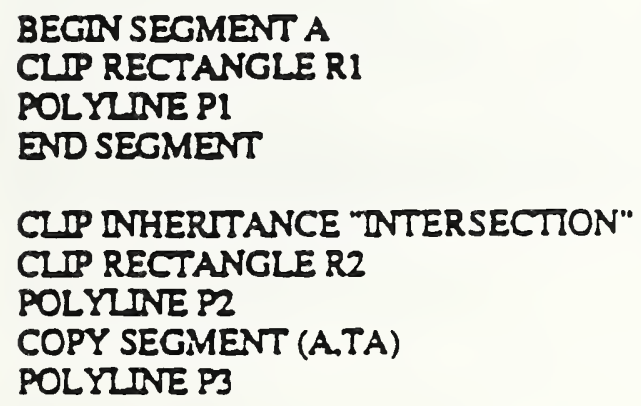

$P 2$ and $P 3$ are taansformed by $R 2$. P1 is transformed by $R 2$ (combined with) TA(R1). This may be an 8.sided convex polygor if TA causes rotation and skewing.

This composition of clipping rectangles continues, however many levels deep the segment hierarchy is nested. For example

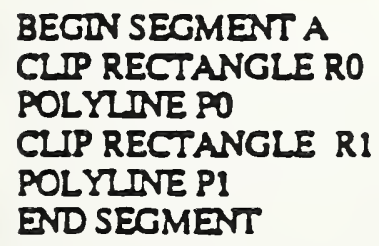


COPY SEGMENT (B.TB)

POLYLNE P3

The effective elipping "rectangles" are:

for P1: $T B(R 2$ intersection $T A(R 1))$ intersection $R 3$

for $P 2: \quad T B(R 2)$ intersection $R 3$

for P3: R3

for PO: $T B(R 2$ inlessection $T A(R 0))$ intersection $R 3$

From this example it can be seen that the effective clipping "rectangle" can in fact be an arbirary convex polygon. Annex $D$ contains recomnended fallback for interprevers which cannot perform such clipping.

Segment Transformations are never applied to clipping boundaries. The default value for CLIP INHERITANCE is 'state_list'.

\subsubsection{Save and Restore Primitive Context}

Two elements are provided to save and restore $a$ contexh that is astributes and control clements. This capability allows a list of acribuses and control elements to be stored in the metafile which can be referenced by name at a later point in the metarile. This capability can be used to save and restore atributes and control elements in conjunction with opening and closing segmenis.

-

Page 41

Add the following text after the state diagram

NOTE. Many eiements allowed in state PO can also occur in the METAFILE DEFAULTS REPLACEMENT. . 
Add the following table following the state diagram

Table 3.1: CGM Elements by their allowed states

\begin{tabular}{|c|c|c|c|c|c|c|c|c|c|}
\hline CGM & & & & & CGM & States & & & \\
\hline Elements & PCS & MDS & $\begin{array}{l}D R \\
\text { (4) }\end{array}$ & GSS & PDS & POS & TOS & LSS & FOS \\
\hline BEGIN METAFILE (sole !) & & & & & & & & & \\
\hline BEGIN PICTURE & $\mathbf{X}$ & $\bar{X}$ & & & & & & & \\
\hline BEGIN PICTURE BODY & & & & & $\bar{X}$ & & & & \\
\hline END PICTURE & & & & & & $X$ & & & \\
\hline BEGNN SEGMENT & & $\underline{X}$ & & & & $x$ & & & \\
\hline END SEGMENT & & & & $\bar{x}$ & & & & $x$ & \\
\hline END METAFILE & $\mathbf{x}$ & & & & & & & & \\
\hline METAFILE VERSION & & $\bar{x}$ & & & & & & & \\
\hline METAFILE DESCRIPTION & & $\bar{x}$ & & & & & & & \\
\hline VDCTYPE & & $\bar{x}$ & $\mathrm{x}$ & & & & & & \\
\hline DNTEGER PRECISION & & $\bar{x}$ & $\bar{x}$ & & & & & & \\
\hline REAL PRECISION & & $\bar{X}$ & $\bar{x}$ & & & & & & \\
\hline DNDEX PRECISION & & $\bar{X}$ & $\bar{x}$ & & & & & & \\
\hline COLOUR PRECISION & & $\bar{x}$ & $\bar{X}$ & & & & & & \\
\hline $\begin{array}{l}\text { COLOUR INDEX PRECISION } \\
\text { NAME PRECISION }\end{array}$ & & $\begin{array}{l}\bar{X} \\
\mathbf{X}\end{array}$ & $\begin{array}{l}\bar{x} \\
\bar{x}\end{array}$ & & & & & & \\
\hline MAXIUMUM COLOUR INDEX & & $\bar{x}$ & $\bar{X}$ & & & & & & \\
\hline COLOUR VALUE EXTENT & & $\bar{x}$ & $\bar{x}$ & & & & & & \\
\hline METAFILE ELEMENT LST & & $\mathbf{x}$ & & & & & & & \\
\hline METAFILE DEFAULTS REPL & & $\bar{X}$ & & & & & & & \\
\hline FONT LIST & & $\bar{x}$ & $\mathbf{X}$ & & & & & & \\
\hline CHARACIER SET LIST & & $\bar{x}$ & $\bar{x}$ & & & & & & \\
\hline CHAR CODING ANNOUNCER & & $\bar{x}$ & $\bar{x}$ & & & & & & \\
\hline METAFILE CATEGORY & & $\bar{x}$ & $\bar{x}$ & & & & & & \\
\hline MAXLMUM VDC EXTENT & & $\bar{X}$ & $\bar{x}$ & & & & & & \\
\hline SEGMENT PRIORTYY EXTENT & & $\bar{X}$ & $\bar{x}$ & & & & & & \\
\hline SCALING MODE & & & $\bar{X}$ & & $\underline{X}$ & & & & \\
\hline COLOUR SELECTION MODE & & & $\bar{x}$ & $\mathbf{x}$ & $\bar{x}$ & $\bar{x}$ & & $\mathrm{x}$ & \\
\hline LNE WIDTH SPEC MODE & & & $\bar{x}$ & $\bar{x}$ & $\bar{x}$ & $\bar{x}$ & & $\bar{X}$ & \\
\hline MARKER SLZE SPEC MODE & & & $\mathrm{X}$ & $\mathrm{X}$ & $\bar{x}$ & $\bar{x}$ & & $\bar{x}$ & \\
\hline EDGE WIDTH SPEC MODE & & & $\bar{X}$ & $\bar{x}$ & $\bar{x}$ & $\bar{x}$ & & $\bar{x}$ & \\
\hline VDC EXTENT & & & $\bar{x}$ & & $\bar{x}$ & & & & \\
\hline BACKGROUND COLOUR & & & $\bar{X}$ & & $\bar{x}$ & & & & \\
\hline DEVICE VIEWPORT & & & $\bar{x}$ & & $\bar{x}$ & & & & \\
\hline DEVICE VIEWPORT MAPPING & & & $\bar{x}$ & & $\bar{x}$ & & & & \\
\hline DEYICE YPORT SPEC MODE & & & $\mathbf{X}$ & & $\bar{X}$ & & & & \\
\hline LNE REPRESENTATION & & & $\bar{x}$ & & $\bar{X}$ & & & & \\
\hline MARKER REPRESENTATION & & & $\bar{x}$ & & $\mathbf{x}$ & & & & \\
\hline TEXT REPRESENTATION & & & $\bar{x}$ & & $\bar{x}$ & $\cdot$ & & & \\
\hline FILL REPRESENTATION & & & $\bar{x}$ & & $\bar{x}$ & & & & \\
\hline EDGE REPRESENTATION & & & $\mathrm{X}$ & & $\bar{X}$ & & & & \\
\hline VDC INTEGER PRECISION & & & $\bar{x}$ & $\mathbf{x}$ & & $\bar{X}$ & & $\bar{x}$ & $\mathrm{X}$ \\
\hline VDC REAL PRECISION & & & $\mathrm{x}$ & $\bar{x}$ & & $\mathrm{X}$ & & $\bar{x}$ & $\bar{x}$ \\
\hline AUXILLARY COLOUR & & & $x$ & $\bar{x}$ & & $\mathrm{X}$ & $\bar{x}$ & $\bar{x}$ & $\bar{x}$ \\
\hline TRANSPARENCY & & & $x$ & $\bar{x}$ & & $\bar{x}$ & $\bar{x}$ & $\bar{x}$ & $\bar{x}$ \\
\hline
\end{tabular}




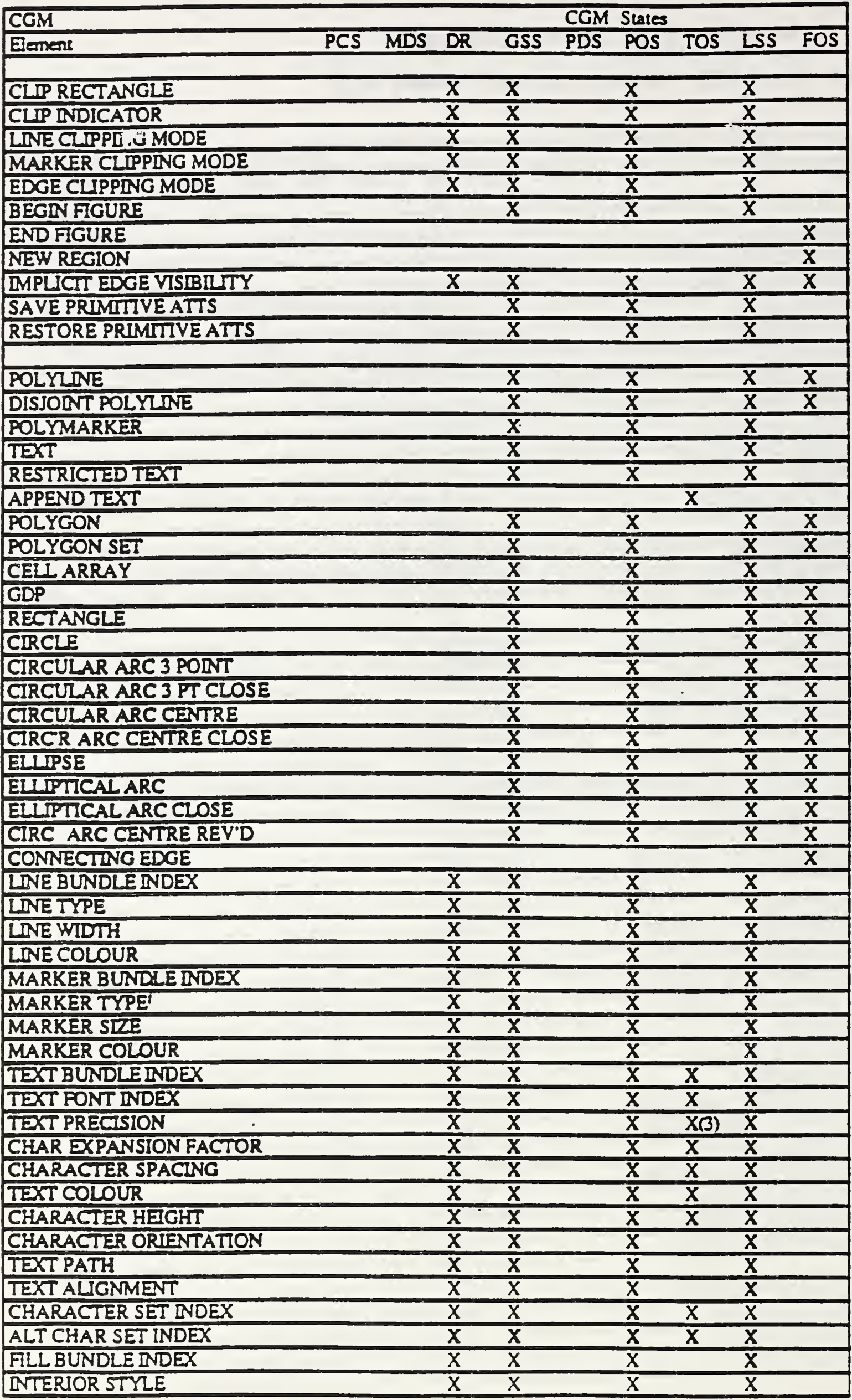




\begin{tabular}{|c|c|c|c|c|c|c|c|c|c|}
\hline CGM & & & & & CGM & Slates & & & \\
\hline Element & PCS & MDS & $\overline{D R}$ & GSS & PDS & POS & TOS & LSS & FOS \\
\hline FIL LOLOUR & & & $\bar{x}$ & $\bar{x}$ & & $\bar{X}$ & & $\overline{\mathbf{X}}$ & \\
\hline HATCh INDEX & & & $\bar{x}$ & $\bar{x}$ & & $\frac{\pi}{x}$ & & $\bar{x}$ & \\
\hline PATTERN INDEX & & & $\bar{x}$ & $\bar{x}$ & & $\bar{x}$ & & $\bar{x}$ & \\
\hline EDGE BUNDLE INDEX & & & $\bar{x}$ & $\overline{\mathbf{x}}$ & & $\bar{x}$ & & $\overline{\mathbf{X}}$ & $\mathbf{X}$ \\
\hline EDGE TYPE & & & $\bar{x}$ & $\bar{x}$ & & $\bar{x}$ & & $\bar{x}$ & $\bar{x}$ \\
\hline EDGE WDTH & & & $\bar{x}$ & $\bar{x}$ & & $\bar{x}$ & & $\bar{x}$ & $\bar{x}$ \\
\hline EDGE COLOUR & & & $\bar{x}$ & $\bar{x}$ & & $\bar{X}$ & & $\overline{\mathbf{X}}$ & $\bar{x}$ \\
\hline EDGE VISBRITY & & & $\overline{\mathbf{X}}$ & $\bar{x}$ & & $\bar{x}$ & & $\bar{x}$ & $\overline{\mathbf{X}}$ \\
\hline FILL REFERENCE POINT & & & $\bar{x}$ & $\bar{x}$ & & $\bar{x}$ & & $\bar{x}$ & \\
\hline PATTERNTABLE & & & $\bar{x}$ & & $\bar{X}$ & $\bar{x}$ & & & \\
\hline PATTERN SIZE & & & $\bar{x}$ & $\overline{\mathbf{X}}$ & & $\bar{X}$ & & $\bar{x}$ & \\
\hline COLOUR TABLE & & & $\bar{x}$ & & $\bar{x}$ & $\bar{x}$ & & & \\
\hline ASPECT SOURCE FLAGS & & & $\bar{x}$ & $\overline{\mathbf{X}}$ & & $\frac{\pi}{x}$ & & $\bar{X}$ & $X(2)$ \\
\hline PICK IDENTIFIER & & & $\bar{x}$ & $\bar{x}$ & & $\bar{x}$ & & $\bar{x}$ & \\
\hline ESCAPE & $\bar{X}$ & $\bar{x}$ & & $\bar{x}$ & $\bar{x}$ & $\overline{\mathbf{X}}$ & $\overline{\mathbf{X}}$ & $\overline{\mathbf{X}}$ & $\bar{x}$ \\
\hline MESSAGE & $\bar{x}$ & $\bar{x}$ & & $\overline{\mathbf{X}}$ & $\bar{x}$ & $\overline{\mathbf{X}}$ & & $\bar{x}$ & $\bar{x}$ \\
\hline APPLCATION DATA & $\overline{\mathbf{x}}$ & $\overline{\mathbf{X}}$ & & $\bar{x}$ & $\bar{x}$ & $\bar{x}$ & & $\bar{x}$ & $\bar{x}$ \\
\hline COPY SEGMENT & & & & $\bar{x}$ & & $\bar{x}$ & & $\bar{X}$ & \\
\hline INHERTANCE FILTER & & & $\overline{\mathbf{X}}$ & $\bar{x}$ & & $\overline{\mathbf{x}}$ & & $\bar{x}$ & \\
\hline CLIP DNHERTTANCE & & & $\mathbf{x}$ & $\mathbf{x}$ & & $\mathrm{x}$ & & $\mathbf{x}$ & \\
\hline SEGMENT TRANSFORMATION & & & $\overline{\mathbf{X}}$ & $\bar{x}$ & & & & $\mathbf{x}$ & \\
\hline SEGMENT HICHLIGHTING & & & $\bar{x}$ & $\bar{x}$ & & & & $\bar{x}$ & \\
\hline SEGMENT DISPLAY PRIORTTY & & & $\overline{\mathbf{X}}$ & $\bar{x}$ & & & & $\bar{X}$ & \\
\hline SEGMENT PICK PRIORITY & & & $\bar{X}$ & $\bar{x}$ & & & & $\overline{\mathbf{X}}$ & \\
\hline PDXELARRAY & & & & & & $\overline{\mathbf{X}}$ & & & \\
\hline
\end{tabular}

$\begin{array}{ll}\text { PCS } & \text { Picture Closed State } \\ \text { MDS } & \text { Metarile Description State } \\ \text { DR } & \text { Default Replacement Mode } \\ \text { GSS } & \text { Global Segment State } \\ \text { PDS } & \text { Picture Description State } \\ \text { POS } & \text { Picrure Open State } \\ \text { TOS } & \text { Text Open (Parial text) State } \\ \text { LSS } & \text { Local Segment State } \\ \text { FOS } & \text { Figure Open State }\end{array}$

Noves:

1: $\quad$ BEGDN METAFILE is the only element allowed in the state Metrilie Closed

2: Only Edge ASFs are allowed in Figure Open Stase

3: Use of TEXT PRECISION in text open state is permined, howeve the intended result is not well defined ans such usage is likely wo lead to unpredictable results.

4: Defauits replacement mode is not acrually a metarile state, but is ineluded in this table for completeness.

Page 42

Sub-clause 5.1: Add the following after the ninth paragraph which stars with the sentence: "The Exicma Elements....":

The Segment Elements (sce 5.10) provide for the grouping and manipulation of elements. 
Sub-clause 5.1: Add the following at the end of the table of abbreviations of data type names:

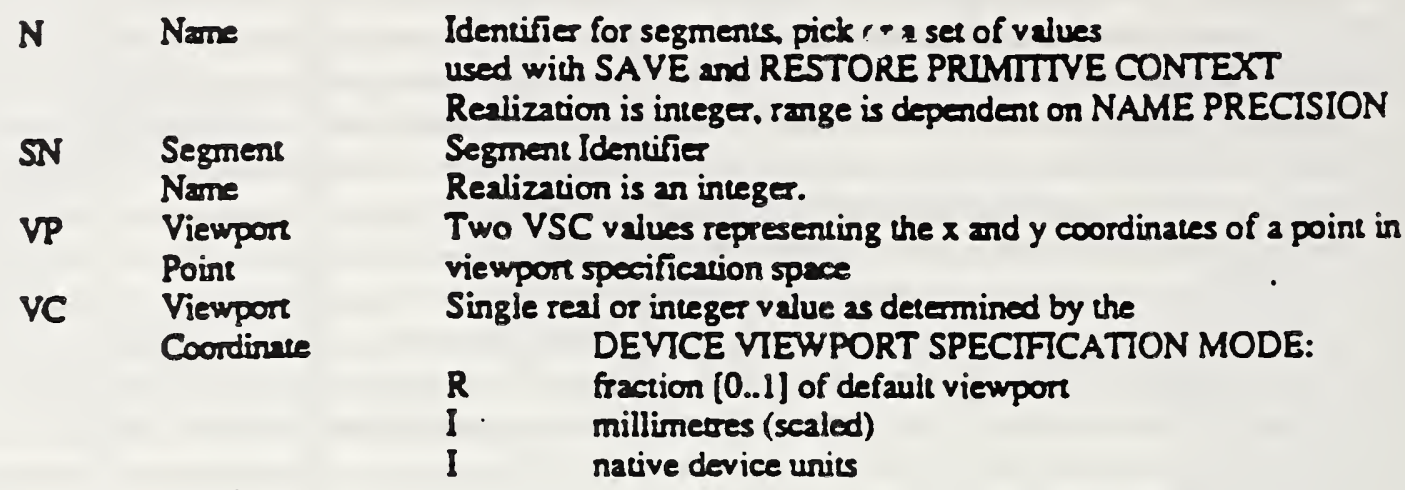

Page 46

Add the following sub-clauses after sub-clause 5.2.5:

\subsubsection{BEGN SEGMENT}

Parameters:

Segmens Identifie (N)

Descriptlon:

This element demareates the start of a segment All subsequent elements until the next END SEGMENT will belong to this segmeni.

Reference:

42

4.12 .3

\subsubsection{END SEGMENT}

Parameters:

Nose

Description:

Subsequent elements will no longer belong wo segment.

\section{Refereace:}

4.2

Page 47

Add the following at the end of the Description section of sub-clause 53.1

The CGM as derined in ISO 8632/1-1987/Add.1 is version two (2).

Page 50

- Sub-clause 53.11: Add the following shorthand names at the end of the list given in the second paragraph of the 'Description': 
EXTENDED-PRDMITVES SET

VER.2-STATIC-GKSM SET

Page 55

Add the following sub-clauses after sub-clause 53.15:

\subsubsection{NAME PRECISION}

Parameters:

The form of the paramter depends on the specific encoding.

Description:

The precision for operands of data type name $(N)$ is specified for subsequent data of type $N$. The precision is defined as the field wideh measured in units applicable to the specific encoding.

\section{Reference:}

43

\subsubsection{MAXIMUM VDC EXTENT}

Parameters:

first come (P)

second come (P)

Descriptloa:

The two comers define a reetangulas extent in VDC space which bounds the values of the VDC EXTENT elements which may be found in the menafile. It may be, but need not be, a closest bound in the sense that it exactly equals the union of the extens recangles in the mesafile.

\section{Refereace:}

4.4.4

\subsubsection{SEGMENT PRIORITY EXTENT}

Parameters:

minisnum extent (I)

maximum extent (I)

Descriptlon:

The paramesers represent an extent which bounds the segment display and pick priority values which will be encountered in the metarle. It need not represent the exact priorities in the metarile. The lowest display priority is zero.

\section{References:}

4.12.4.3

4.12.4.4

Page56

Add the following note to the end of sub-clause 5.4.1 (SCALNG MODE)

NOTE: If both device viewpor and scaling mode appeas in the same metafile then the last specified is used. If ncither appear then the default values for device vicwport lake precedence. 
Add the following sub-clauses after sub-clause 5.4.7:

\subsubsection{DEVICE VIEWPORT}

Parameters:

first comes (VP)

second comer (VP)

Description:

The two parameters define the opposite comers of a rectangular viewport on the device's drawing surface.

These parameters are specinied by the unit system selected by DEVICE VIEWPORT SPECIFICATION MODE

The effective viewport is that area of the drawing surface onto which the VDC extent rectangle is mapped. If the crrrens DEVICE VIEWPORT MAPPING forces isotropic mapping. and the aspect ratio is not equal to that of the device viewport, the effective viewport will be smaller than the specified viewport on one or the olher axis (but not both).

If the current DEVICE VIEWPORT MAPPING does not force isotropic mapping, the effective viewport will be the same as the specified viewpors. If the Device Viewport exceeds the available drawing surface, the Device Viewpon is still used to determine the VDC-io-Device mapping.

Mirroring or 180 degree rotation of the image may be achieved by specifying the comers in some way other than that the first is below and to the left of the second.

NOTE: If both device viewport and sealing mode appear in the same metarile then the last specified is used. If neither appear then the default values for device viewpor lake precedence where these are allowed in the same category.

\section{Reference:}

4.4.7

\subsubsection{DEYICE VIEWPORT SPECIFICATION MODE}

Parameters:

VC specifie $\quad$ (One of: fraction of dawing suriace millimertes with sealefactor. physical device uniks)(E)

Metric scale factor

(R)

\section{Description}

This element detemines how subsequent elements using the data type VC (Viewport Coordinate) or VP (Viewpor Poind) will be defined.

These parameters may be specified in one of three modes: fraction of dawing surface; millimetres with scale factor, or physical device units.

When the VC specinies is 'traction of drawing surface', the value $(0.0,0.0)$ corresponds to the lower left comer and the value $(1.0,1.0)$ corresponds to the upper righs corres of the default device viewport (The default device viewpors is the largest urrotaicd receangular area visible on the drawing surface.) Numbers outside of the sange [0.0..1.0] may be specitied (see DEVICE VIEWPORT). In this case the merric scale factor is ignoted.

When the VC specinier is 'millimetres svith sealefactor', the metric seale factor parameter represents the distance (in millimetres) on the drawing surface corresponding to one unit in VP space. One unit in VP space represents one millimetre multiplied by the merric scale factor. The value $(0.0)$ corresponds to the lower left comer and the values increase posicively to the right and upwards.

- When the VSC specifier is 'physical device units', the native units and handedress of the physical device are used ts this case the metric scale factor is ignored. 
Mecric sealing with a scale factor provides a device-independent means of generating output at a known seale factor. In metric mode. a scale factor of 1.0 indicates that the VC are in units of millimetres; a scale factor of 0.0254 would imply a VSC of one thous and per inch. The oniy allowed dala type for physical device units is integer.

Reference:

4.4.7

\title{
5.4.10 DEVICE VIEWPORT MAPPING
}

Parameters:

Isocropy Ilag

Horizontal alignment nag

(one of: not foreed foreed XE)

Verical alignment Ilag

(one of: Lefi. Centre RightX(E)

(one of: Bollom, Cente Top)(E).

Descriptlon:

This element determines how the coordinate mapping is derived from the VDC EXTENT and the specificd

DEVICE VIEWPORT. The remaining parameters are only significant if isotropy is forced by the first parameter. If so, the effective viewpon is generally smalle than the specified viewport and these parameters decrmine how it will be positioned within the specified viewpon. 'Left' and 'bottom' are interpreted as being wowards the 'first come' of the specinied DEVICE VIEWPORT. segardless of any miroring or rotation of the viewpon on the physical device.

\section{Reference:}

\subsection{7}

\subsubsection{LNE REPRESENTATION}

Parmeters

\author{
lixe burdle index (IX) \\ line rype indicalos (DX) \\ line wideh specifie \\ if line width specification mode is 'absolute", \\ absolute line width (VDC) \\ if line width spciricarion mode is 'sealed'. \\ line width seale factor $(R)$
}

line colour

if the colour selection mode is "indexed".

line colour index (CI)

if the colour selection mode is 'direct.

line colour value $(C D)$

\section{Description:}

In the line bundle table, the given line bundle index is associated with the specific parameters.

Lixe type is specined and behaves as indicaled in the LDNE TYPE atribute element

Line width is derined in the current LDVE WIDTH SPECIFICATION MODE and is stored in the bundle table long with that mode. Thus the derinition is immune to subsequent changes in the specinication mode.

Line colour is defined in the current COLOUR SELECTION MODE and is stored in the bundle table along with thas mode. Thus the definition is immune to subsequent changes to the selection mode.

Which aspects are used depends on the corresponding ASFs. see the ASPECT SOURCE FLAG ciement

Reference:

- 4.4 .8

\subsubsection{MARKER REPRESE.ITATION}




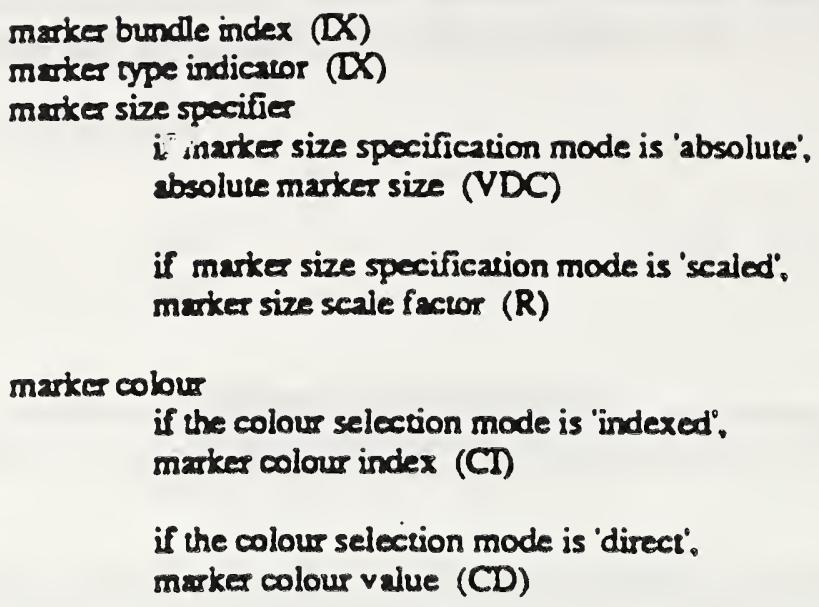

Deseription:

In the marker bundle table the given markex bundle index is associated with the specified parameters.

Marker type is specified and behaves as indicated in the MARKER TYPE aturibute element.

- Marker size is defined in the current MARKER SIZE SPECIFICATION MODE and is stored in the bundle table ajong with thas mode. Thus the defirition is immune to subsequent changes in the spocification mode.

Marker colour is defined in the current COLOUR SELECTION MODE and is stored in the bundle table along with thas mode. Thus the definition is immune to subsequent changes to the selection mode.

Which aspects are used depends on the corresponding ASFs, see the ASPECT SOURCE FLAG element.

\section{Refereace:}

4.4 .8

\section{S.4.13 TEXT REPRESENTATION}

\section{Parameters:}

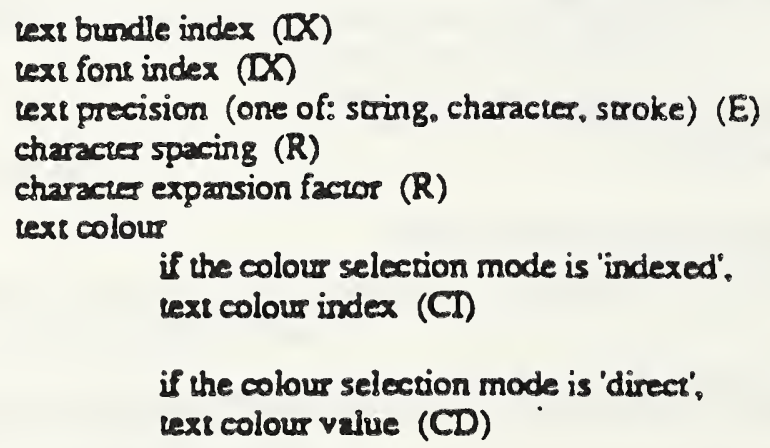

Is the lext bundle table, the given text bundle index is associated with the specified parameters.

Text fon index is specified and behaves as indicated in the TEXT FONT INDEX arribute element.

Text precision is speciried and behaves as indicated in the TEXT PRECISION attribute element.

Character spacing is specified and behaves as indicated in the CHARACTER SPACING atribute elcment.

- Character expansion factor is specified and behaves as indicated in the CHARACTER EXPANSION FACTOR astribuse eiement. 
Text colour is derined in the current COLOUR SELECTION MODE and is stored in the bundle table along with that mode. Thus, the definition is immune to subsequent changes 10 the selection mode.

Which aspects are used depends on the corresponding ASFs, see the ASPECT SOURCE FLAG element

\section{Reference:}

\subsection{8}

\subsubsection{FLL REPRESENTATION}

\section{Parameters:}

fill area bundle index (DX)

interior style (one of: hollow, solid. panern.hatch empry)(E)

fill colour

if the colour selection mode is "indexed",

fill colour index (CI)

if the colour selection mode is 'direct:

ill colour value $(C D)$

hatch index $(D X)$

partern index (DX)

\section{Description:}

In the fill bundle table. the given fll bundle index is associated with the specified parametcrs.

Interior style is specified and behaves as indicated in the INTERIOR STYLE atribute element.

Fill colour is defined in the current COLOUR SELECTION MODE and is stored in the bundle tabie along with that mode. Thus, the definition is immune to subsequent changes io the selection mode.

Hutch index indicator is specinied and behaves as indicared in the HATCH DNDEX atribute element.

Parem index indicator is specisied and behaves as indicared in the PATTERN DNDEX astribute element

Which appects are used depends on the corresponding ASFs. see the ASPECT SOURCE FLAG element.

\section{Reference:}

4.4.8

\subsubsection{EDGE REPRESE.VTATION}

\section{Parameters:}

$$
\begin{aligned}
& \text { edge bundle index (DX) } \\
& \text { edge type indicator (DX) } \\
& \text { edge wideh specifie. }
\end{aligned}
$$

if edge width specification mode is 'absoluse'.

absolure edge width (VDC)

if edge wideh spcification mode is 'scaled". edge width scale factor $(R)$

edge colour

if the colour seloction mode is "indexed".

edge colour index (CI)

if the colour sclection mode is 'direct.

edge colour value (CD)

\section{Description:}

In the edge bundle table. the given cdge bund!e index is associated with the specified parametcrs. 
Edge type is specified and behaves as indicated in the EDGE TYPE atribute element

Edge width is defined in the current EDGE WIDTH SPECIFICATION MODE and is stored in the bundle table along with thas mode. Thus the definitios is immune to subsequent changes in the specification mode.

Es- colour is defined in the current COLOUR SELECTION MODE and is 'red in the bundle table along with that mode. Thus, the definition is immune to subsequent changes to the selection mode.

Which aspects are used depends on the corresponding ASFs, see the ASPECT SOURCE FLAG element.

\section{Reference:}

4.4.8

Page 61

Add the following sub-ciauses after sub-clause 5.5 .6

\subsubsection{LINE CLIPPING MODE}

Parameters

mode (one of: locus, shape, locus then shape) (E)

Descriptlon

The Line Clipping Mode is set to the value specified.

Reference:

45.2

\subsubsection{MARKER CLIPPING MODE}

Parameters

mode (one of: locus, shape, locus then shape) (E)

Description

The Marker Clipping Mode is set to the value specified.

Reference:

4.5 .2

\section{5 .9 EDGE CLIPPI.VG .MODE}

Parameters

mode (one of: locus, shape, locus then shape) (E)

Description

The Edge Clipping Mode is set to the value specified.

Reference:

4.5 .2

\subsubsection{BEGIN FIGURE}

Parameters:

none

Description:

This is the first eiement of a closed figure. All subsequent elements until the next END FIGURE will be par of the closed nigure.

Reference: 


\subsubsection{END FIGURE}

Parameters:
nome
Desc:piton:

This element terminates the current closed figure.

If the current region has not yes been closed by a preceding NEW REGION or CONNECTLNG EDGE elemenh and the last point of the last line element is not coincident with the cursent closure point then the current subregion is closed by a line segment cornecting the last point of the preceding line element wo the current closure point. This line becomes a part of the boundary specificarion. If the region which has been previously closed is empry, or the last point of the last line element is coincident with the current closure point, then no line segment is generated by this element.

Reference:

4.6.8

\subsubsection{NEW REGION}

Parameters:

none

\section{Description:}

This element is used for control of subregion consoruction within closed figures.

If the current region has not yes been closed by a preceding NEW REGION or CONNECTLNG EDGE clement and the last point of the last line element is not coincident with the current closure point. then the current subregion is closed by a line segment comecting the last point of the preceding line element to the current closure poist. This line becomes a part of the boundary specification. If the region which has been previously closed is empty, or the last point of the last line element is coincident with the current closure point then no line segment is generated by this element.

The first point of the next line element following a NEW REGION element becomes the new closure point staring a new subregion.

Reference:

4.6.8

\subsubsection{SAVE PRIMITIVE CONTEXT}

Parameters:

Conrext rame (N)

Descriptlon:

This element allows for the grouping and identification of the set of current values of the attribute and control elements listed in the list bejow as a single named encity.

Groups of elements may be saved in a picrure or segment using the context name.

The arribure and control elemenis which may be saved by SAVE PRIMITTVE CONTEXT and restored by RESTORE PRIMITIVE CONTEXT are:

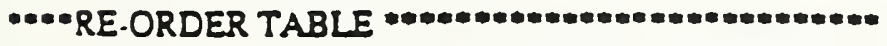

CHARACTER CODING ANNOUNCER

AUXILIARY COLOUR (NoIe !)

EDGE BUNDLE INDEX

- CLIP RECTANGLE (Note 3)

EDGE TYPE

CLIP INDICATOR

EDGE WIDTH (Nole 2) 


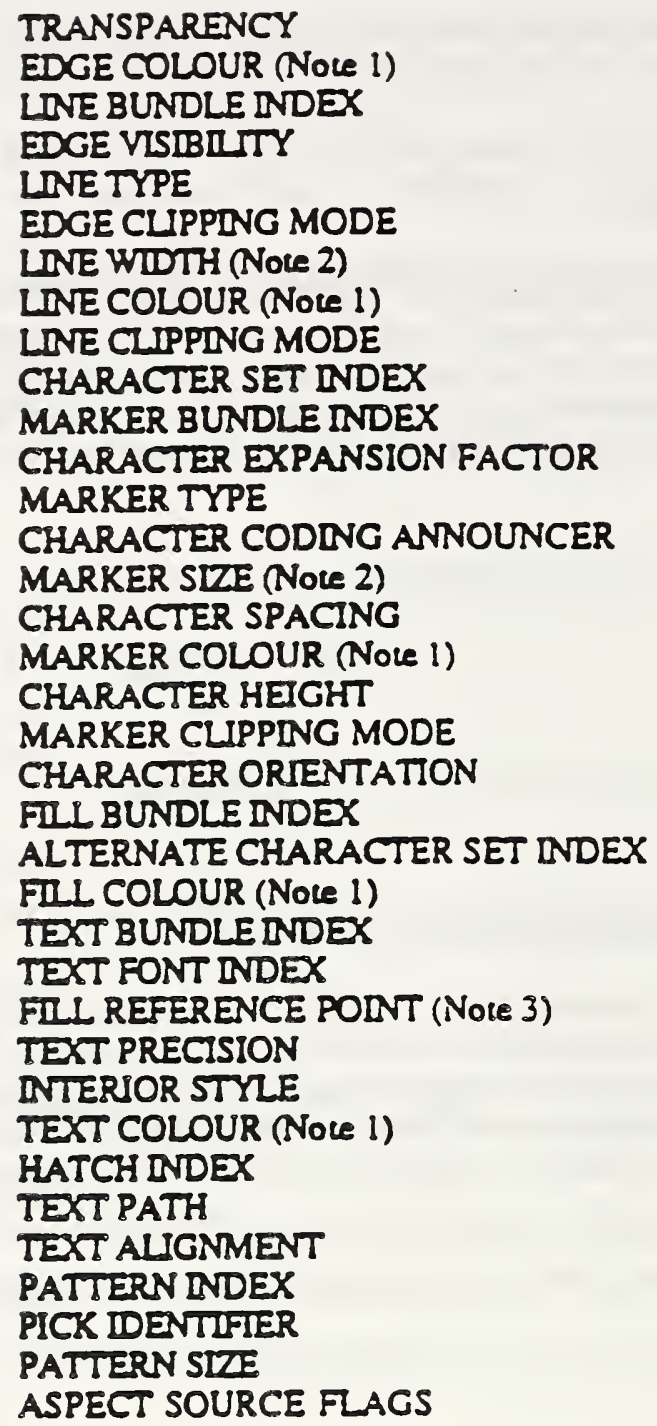

NOTES:

1: The COLOUR SELECTION MODE in which this value was last set is also recorded.

2. The corresponding specification mode in which this value was last set is also recorded.

3. The VDC TYPE in effect when these values are saved is also recorded

Reference:

4.12 .6

\subsubsection{RESTORE PRIMITIVE CONTEXT}

\section{Parameters:}

Convext name (N)

Description:

The astribuse and control set recorded in the menfile with the last SAVE PRDMITIVE CONTEXT eiement are recalled on inierpretation.

Reference:

$$
4.12 .6
$$


Add the following text wo the end of the second paragraph of sub-clause 5.63

These instructions for the acrual displayed position of a marke ' only apply to MARKER CLIPPING MODE locus'.

Page 78

Add the following sub-clause after sub-clause 5.6.19

\subsubsection{CIRCULAR ARC CENTRE REVERSED}

\section{Parameters:}

centepoint (P)

DX_star. DY_starh DX_end. DY_end (4VDC)

gadius (VDC)

\section{Description:}

A circular arc is drawn which is derined as follows:

DX_start and DY_star define a start vector. and DX_end and DY_end define an end vector. The tails of these veciors are placed on the centrepoint $A$ star ray and end ray are derived from the star and end vectors. The start and end rays are semi-insinite lines from the centrepoint in the directions of the star and end vectors respectively.

The specified radius and centrepoins derine a circle. The arc is drawn in the negative angular direction (as defined by VDC EXTENT) from the intersection of the circle and the star ray (as oblained by measuring a distance 'radius' along the start ray from the centrepoins) to the intersection of the circle and the end ray.

The are is displayed with current line element auributes.

Valid values of the vector components are those which produce vectors of non-zero length.

Valid values of 'radius' are non-negative VDC.

If the start ray and end ray are coincident is is ambiguous whether the defined arc subtends $\sigma$ degrecs or 360 degrees of central angle (see the specinications for the CIRCULAR ARC CENTRE in arnex D).

\section{Reference:}

4.6

\subsubsection{CONINECTI.YG EDGE}

\section{Parameters:}

none

\section{Description:}

During the construction of a closed figure a line segment consecting the last point of the preceeding line element and the next poins is added to the boundary definition. The next point which must be different from the last point, may be:

1. the first point of the next line element, or

2. the current closure point that is in cases where CONNECTING EDGE is followed by either NEW REGION or END FIGURE.

The appearance of the comecting edge is fully detcrmined by the edge atoributes and EDGE VISIBILTY.

References:

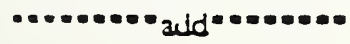


Add the following sub-clauses after sub-clause 5.7 .35

\subsection{PICK IDENTIFTER}

Parameters:

pick identifies (N)

Descriptlon:

The pick identifier value is associated with all of the graphical primitive elements of a segment until the next PICK IDENTIFIER element. Usage of the PICK IDENTIFIER on interpretation is dependent upon the application and on the category of the metarile.

Reference:

4.7.9

Page 100

Add the following sub-clause after sub-clause 5.9:

\subsection{Segment elements}

\subsubsection{Segment control elemedts}

\subsubsection{COPY SEGMENT}

Parameters:

segment identirier

copy transformation matrix:

scaling and rotation portion

$(2 \times 2 \times R)$

transiation portion

$(2 \times 1 \times V D C)$

segment transformation application

(one of: NO, YES) (E)

Description:

The segmens which is indicuted by the segment identifier is referenced at this point in the metafile for copying into the picarre, or into a segment when referenced from a segment on interpretation. With the exception of the segment transformation associated with the copied segment the identinied segment is referred to as the copied segment. The segment arributes of the copied segment are ignored. Whether or not this segment is ignored is controlled by the "segment transformation application" parameter. The segment atributes of the segmens in which the COPY SEGMENT may occur are unchanged by this element

The copy transformation is applied to all primitive elements of the copied segment before they are copied into the open segment. The copy transformation is also applied to clipping rectangles under some circumstances.

The INHERTTANCE FILTER element allows for control of the auribute values which are used when copying segments. This filler controls whether individual auribute values are reapplied to the graphical primitives. The effects of INHERITANCE FILTER are described in Clause 4. The way in which clipping is applied to primitives within a copied segment is controlled by CUIP INHERTTANCE (see Clause 4).

The "segment transformation application" parameter controls whether or not the segment transformation associalted with the copied segment will be applied as an effect of the copy process. If it is, the application of the segmens transformation is never applied to a clip rectangle associated with a copied object.

Reference:

4.12.1

4.12 .5

\subsubsection{INHERITANCE FILTER}

Parameters:

filtcr selection attribute designasor (list elements or groups from: 


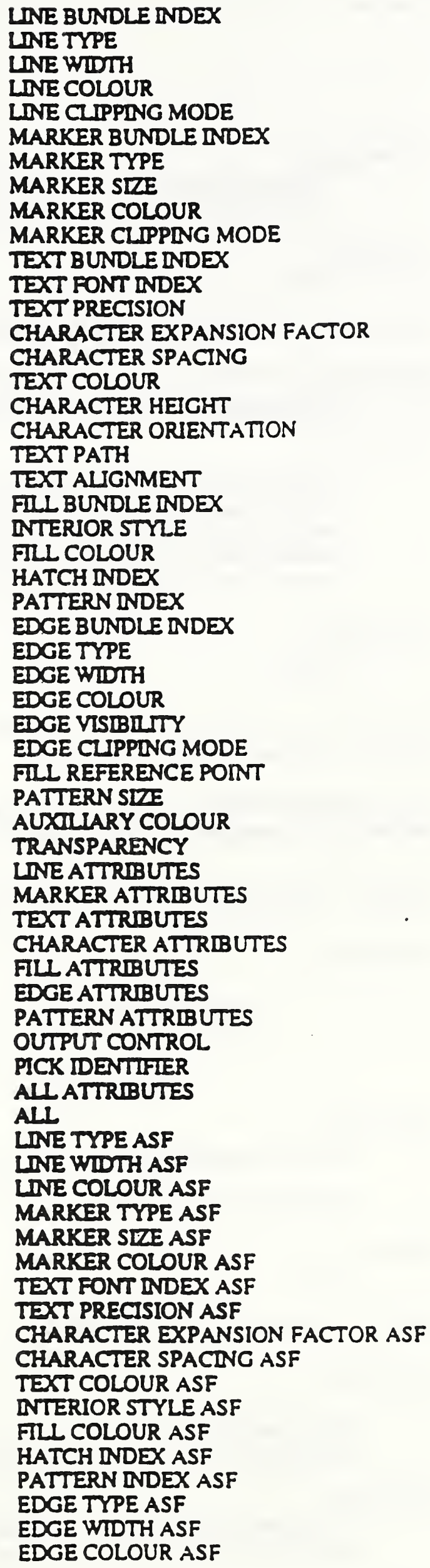




\section{Descriptlon:}

The seting of the inheritance filter is modified for those atributes in the filter selection list According to the setting, atributes are inherited from the current state lists or from the copied segment.

\section{Reference:}

\subsection{2 .5}

\subsubsection{CLIP INHERITANCE}

Parameters:

clip inheritance (one of: state list, intersection)

Description:

The behaviour of clipping as applied to primitives in copied segments is derined. Simple clipping against the current rectangle in the modal stase list is selected by the value 'state_list'. The value 'intersection' not only selects the clip recungle to come from the segment but also enables an "object clipping" feanure. The transformation of clip rectangles and accumulation or composition of multiple ransformed rectangles is enabled depending upon the sertings of CLIP INDICATOR. See Clause 4 for a description of the mechanism.

\section{References:}

4.12.1

4.12 .5

\section{S.10.2 Segment Attribute Elements}

Segment Atribute Elements. if used, shall all appear immediately after BEGN SEGMENT, before the first element of another type. The segment identifies shall refer to the segment in which the elements are contained.

\subsubsection{SEGMENT TRANSFORMATION}

\section{Parameters:}

segment identifier $(\mathbb{N})$

transformation matrix:

sealing and rotation portion $\quad(2 \times 2 \times R)$

translation portion $\quad(2 \times 1 \times V D C)$

Descriptlon:

The segment transformation marrix for the identified segment is ses to the specified parameter.

The default segment transformation is the identity matrix.

\section{Reference:}

\subsubsection{5}

\subsubsection{SEGMENT HIGHLIGHTING}

\section{Parameters:}

segment identifier $(N)$

highlighting (one of: nomal, highlighted) (E)

Description:

The segmens highlightling for the identified segmens is set to the specified value. When the highlighting atribute is set to 'highlighted', the visual appesrance of the segment is interpretation dependent. When the 
highlighting atribute is set to 'normal', the segment is dispiayed according to the segment and primitive uroributes.

\section{Reference:}

4.12.4.2

\subsubsection{SEGMF.NT DISPLAY PRIORITY}

Parsmeters:

Descriptlon:
segment identifies
(N)
segment display priority
(I)

The segment display priority for the identified segment is set wo the specified value.

Segments with higher segment display priority appear to be in front of segments with lower segment display priorities. When the segment display priorivies of two overlapping segments are the same. the order in which they appear is interpretation dependent

\section{Reference:}

4.12.4.3

\subsubsection{SEG.MENT PICK PRIORITY}

\section{Parameters:}

segment identinet

segment pick priority
(N)

(I)

Descriptlon:

The segment pick priority for the identified segment is set to the specified value. The pick priority does not affect the display of segments.

\section{Reference:}

4.12.4.4

Page 103

Clause 6: Add the following at the end of clause 6:

NAME PRECISION

MAXTMUM VDC EXTENT

SEGMENT PRIORTY EXTENT

DEVICE VIEWPORT

DEYICE VIEWPORT SPECIFICATION MODE

DEVICE VIEWPORT MAPPDNG

LDNE REPRESENTATION

MARKER REPRESENTATION

TEXT REPRESENTATION

FILL REPRESENTATION

EDGE REPRESENTATION

LNE CLIPPING MODE

MARKER CLIPPING MODE excoding dependent

VDC EXTENT

$0 . .255$

$0 . .1 .00 .1$.

fraction of drawing surface

forcedleft,bottom

interpreter dependent

intorpseter deperdent

interpreter deperdent

interprever dependent

inserpreter dependent

locus

locus 
EDGE CLIPPING MODE

PICK IDENTHFIER

DNERTTANCE FILTER

CUP DNHERTTANCE

SEGMENT TRANSFORMATION

SEGMENT HIGHLIGHTING

SEGMENT DISPLAY PRIORITY

SEGMENT PICK PRIORITY bass

0

segment

state list

$1,0 \quad 0,1 \quad 0.0$

normal

0

0

Page 104

Add the following clause after sub-clause 7.4

\subsection{Conformance for Version 2 metafiles}

This conformance section defines conformance for metafiles which are 'version 20. A Computer Graphics Metafile (CGM) is said to conform to the standard if it implements precisely all the elements required for a version 2 metarile as defined in this standard. When determining conformance of a CGM, the formal grammar shall take precedence.

Page 123

Add the following to the end of sub-clause D.1:

In a static picare-capture metarile potentially dynamic effects are avoided by limiting the position of elements with such potentially dymamic effects. Thus bundle cable definitions may only appea in the picare descriptor. In a metafile the effects of COLOUR TABLE and PATTERN TABLE are unspecified when they occur in a location with potentially dynamic implications. In metanles which have a version number which is greater than one these eiements may appear in the Picture Descriptor. Use of these elements in the picrure body is discouraged in order to improve the portability and predicrability of CGM exchange.

Poge 125

Add sub-clause D. 3.23

It is recommended that the mandatory elements in the Metafile Descriptor are writuen first in the desriptor and in the following order.

METAFILE VERSION

METAFILE ELEMENT LIST

METAFILE DESCRIPTOR

Page 127

Sub-lause D.4.3: replace the sentence with the following text

DEVICE VIEWPORT, DEVICE VIEWPORT SPECIFICATION MODE DEVICE VIEWPORT MAPPING In the case where the VC specirier in DEVICE VIEWPORT SPECIFICATION MODE is set to either 'millimertes with seaie factor' or 'physical device units' not all interpreters may be able to interpret the DEVICE VIEWPORT element as specified. and the interpreation becomes implementation dependent. Since the CGM does no specify the behaviour of an interpreter an application may wish to control the VDC-to-device mapping by mechanisms external to the CGM picture description for example to include CGM pictures in documents. 
Add the following texe to the end of the sub-clause D.4.4:

Clipping Modes

If inierpreters cannot handle the locus' clipping mode for LINE CLIPPING MODE. MARKER CLUPPING MODE or EI JE CLIPPDNG MODE, then 'ocus plus shape' should be used as a fallback

Page 127

Add the following text to the end of sub-clause D.4.4

If interpreters carnot handle clipping to the parallelogram that could result from using CLIP DNHERTAANCE value 'intersection' the suggested fallback is to clip to the minimal circumscribing rectangie. In cases where multiple parallelograms might be composed (by intersecion) to form a general convex polygon. inteppreters should intersect the circumscribing rectangles to derive an effective clip rectangle.

Poge 127

Add the following text w the end of the APPEND TEXT recommendations:

Changing the TEXT PRECISION in partial text state is likely to lead to unpredictable results. Generators are discouraged from doing this. Interpreters which can otherwise handle text attribute changes in partial text state should ignore this element in that state as a fallback.

Page 128

Sub-ciause D.4.S: Add the following text between CIRCULAR ARC CENTRE CLOSE and Elliptical elements:

CIRCULAR ARC CENTRE REVERSED

If the star ray and and say coincide, it is recommended that the interpreter draw the full circle.

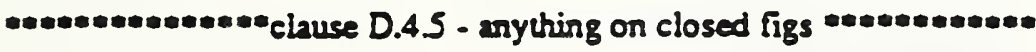

Poge 132

Add the following sub-clause after sub-clause D.4.8

\section{D.4.9 Segment elements}

The restriction of segment atributes to be set only innediatly after the BECNN SEGMENT element and before any other element avoids any dynamic effects. If the output device cannot adjust segment priority on interpretation, segments should be displayed in orde of priority.

Page 133

Sub-clause D5. Charige the sentence to:

.......capabilities listed in the cables below, appropriate to the version of the metafile they want wo suppor.

Page 133

Sub-clause DS. Change the title for Table 5 to:

Table S(a) Suggested minimum capabilities for version 1 metafiles

Poge 133

Sub-elause D.S Add the following table after Table 5(a)

Table S(b) Suggested additional minimum capabilities for version 2 metafiles

Capabllity

.Mnimum Suggested Interpreter Support 
DEVICE VIEWPORT SPECIFICATION MODE DEVICE VIEWPORT MAPPING

\author{
LNE REPRESENTATION \\ MARKER REPRESENTATION \\ TEXT REPRESENTATION \\ FIL REPRESENTATION \\ EDGE REPRESENTATION \\ LDNE CLIPPING MODE \\ MARKER CLIPPING MODE \\ EDGE CLPPING MODE
}

\author{
fraction of drawing surface \\ not forced, forced \\ left centre right \\ bowom, centre lop \\ $S$ entries \\ 5 entries \\ 2 entries \\ $S$ entries \\ 5 entries \\ locus, shape locus then shape \\ locus, shape locus then shape \\ locus, shape locus then shape
}


The following arnex forms a new annex $F$.

\section{F Formal Grammar of the Functional Specification of the CGMADD1 rategory}

\section{F.1 Introduction}

This grammar is a formal definition of a suandard CGM extended symtax. The encoding-independent and the encodingdependent productions are separated, and there are subsections showing the synux of each of the standardized encoding schemes. Details on the encoding of terminal symbols can be found in parts of this Standard that deal with the parcicular encoding schernes.

\section{F.2 Notation used}

\begin{tabular}{|c|}
\hline 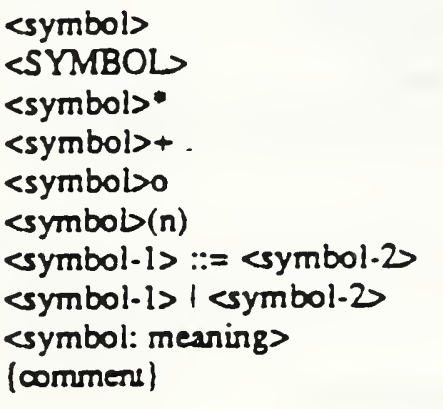 \\
\hline
\end{tabular}

\section{F.3 Detailed grammar}

\section{F.3.1 Metanle structure}

emetafiles

enctarile idenvifies

<mctarile contents>

<exua elements

<picrures

<pictrre identifies

<picrure conteno

<picrure clemeno
- nonecrminal

- terminal

- 0 or more occurrences

- I or more occurrences

- optional (0 or 1 occurrences)

- exactly n occurrences, $n=2.3 . .$.

- symbol-1 has the syntax of symbol-2

- symbol-1 or alternatively symbol-2

- symbol with the stated meaning

- explanation of a symbol or a production

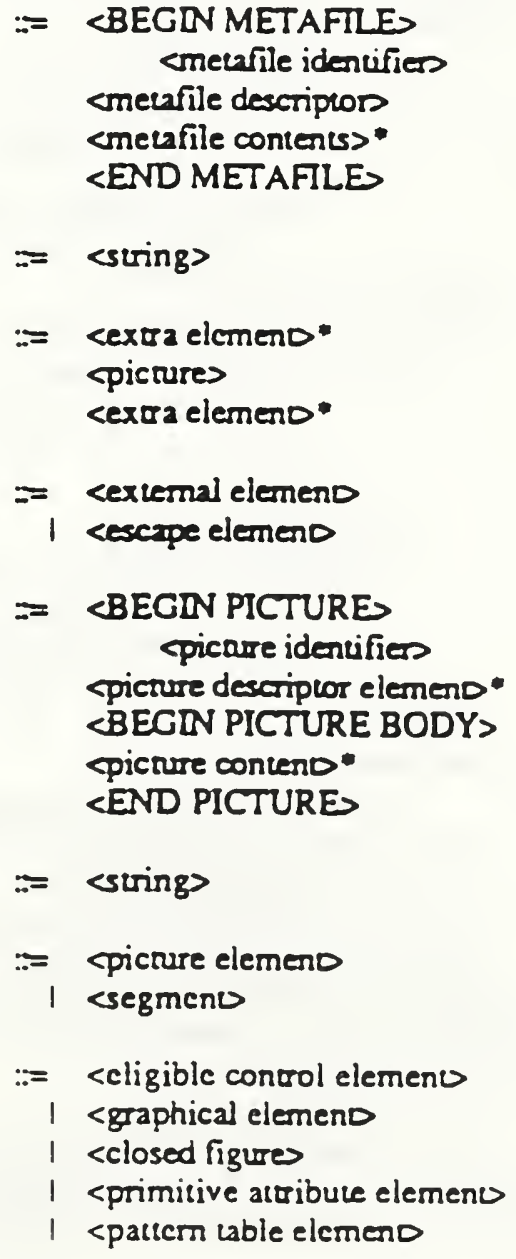




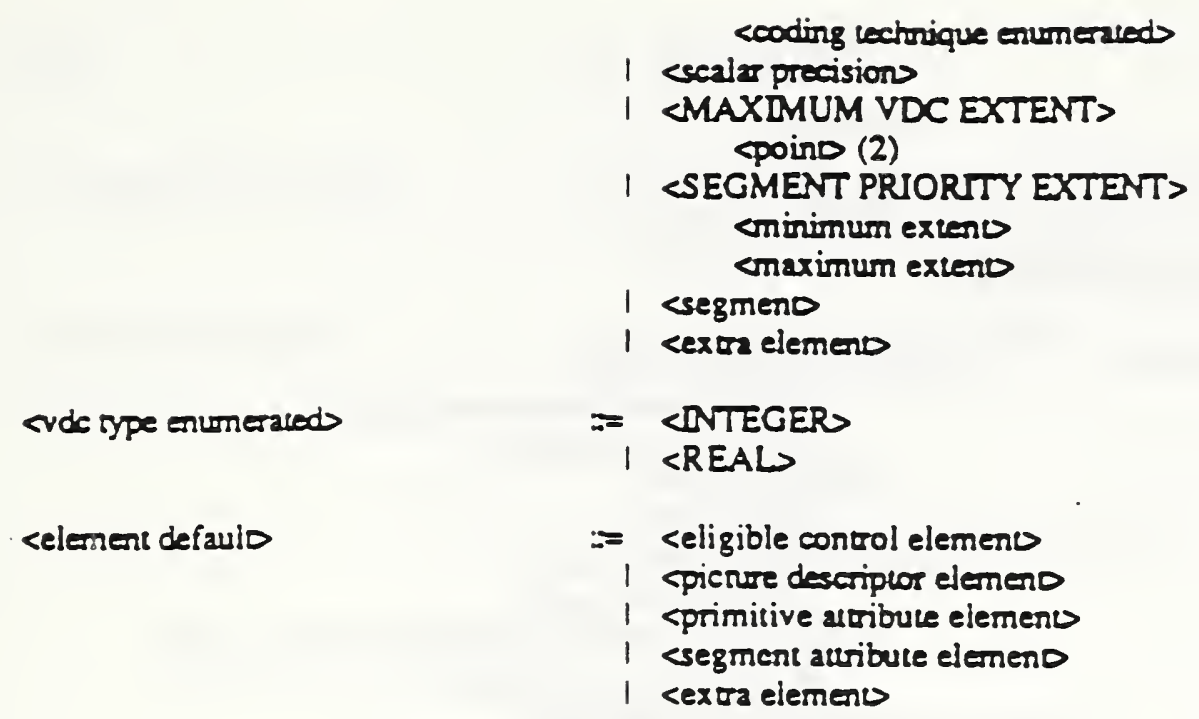

$\ldots+\ldots$ in is 8632 only escape allowed above not exta - what is right?

<fon: name>

<characer set definition

index

standard index vahues eron-negative integers Spositive integers Sprivace index values oregative iniegers <positive index>

<char sel enumerased

<coding lechnique enumerated

<designation sequences

cecalar prevision

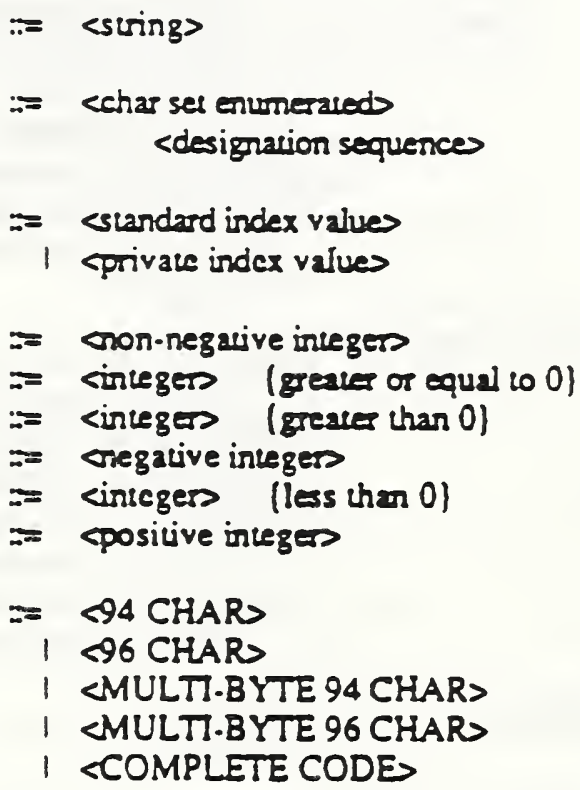




$\begin{array}{ll}\text { <poind } & =\text { orde values (2) } \\ \text { <minimum extent } & ::=<\text { integers } \\ \text { cmaximum extend } & ::=<\text { integers }\end{array}$

F.3.3 Plcture descriptor elements

spicrure descripror elemeno

$=$ ¿SCALNNG MODE

cealing spec mode enumeratod coneric seale factors

1 <VDC EXTENT> Spoino (2)

1 <DEVICE VIEWPORT> Sviewpor poino(2)

1 CDEVICE VIEWPORT SPECIFICATION MODE $<V C$ specifier emumeraiods eneric scale factors

1 <DEVICE VIEWPORT MAPPING> <isotropy flag enumeraleds chorizontal alignment flag emumerated> sverical alignment flag enumeratod>

1 <BACKGROUND COLOUR> ered green blues

1 especifiention element

1 crepresenution elemeno

1 spartem uble elemeno

1 <colour table elemeno

1 cexura elemeno

especificacion elemeno

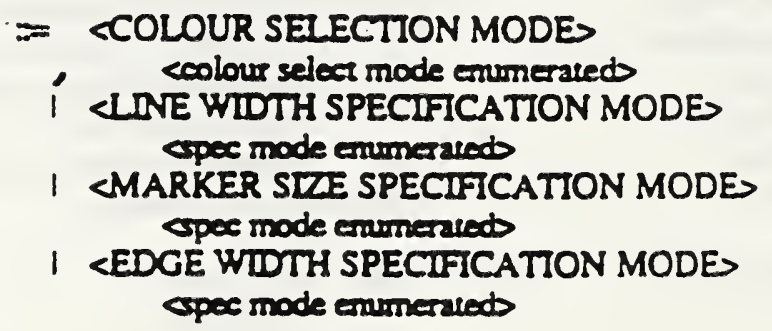

<colour select mode enumerated

$\approx$ CNDEXED

1 <DIRECT>

escaling spec mode enumeraleds

$=$ CABSTRACT

1 <METRIC

emertic scale factor

$=$ creal

<isotropy nag cnumerated

$:=$ QNOT FORCEDS

1 <FORCED>

choriz align llag enumers
$=$ <LEFT>
1 SCENTRE
I <RIGHTS

cver align lag enumers

= ¿BOTTOMS

1 <CENTRE

1 <TOP

spoc mode cnumcrated

$=$ CABSOLUTE

1 <SCALED

sieuport poino

$:=\langle$ pp 


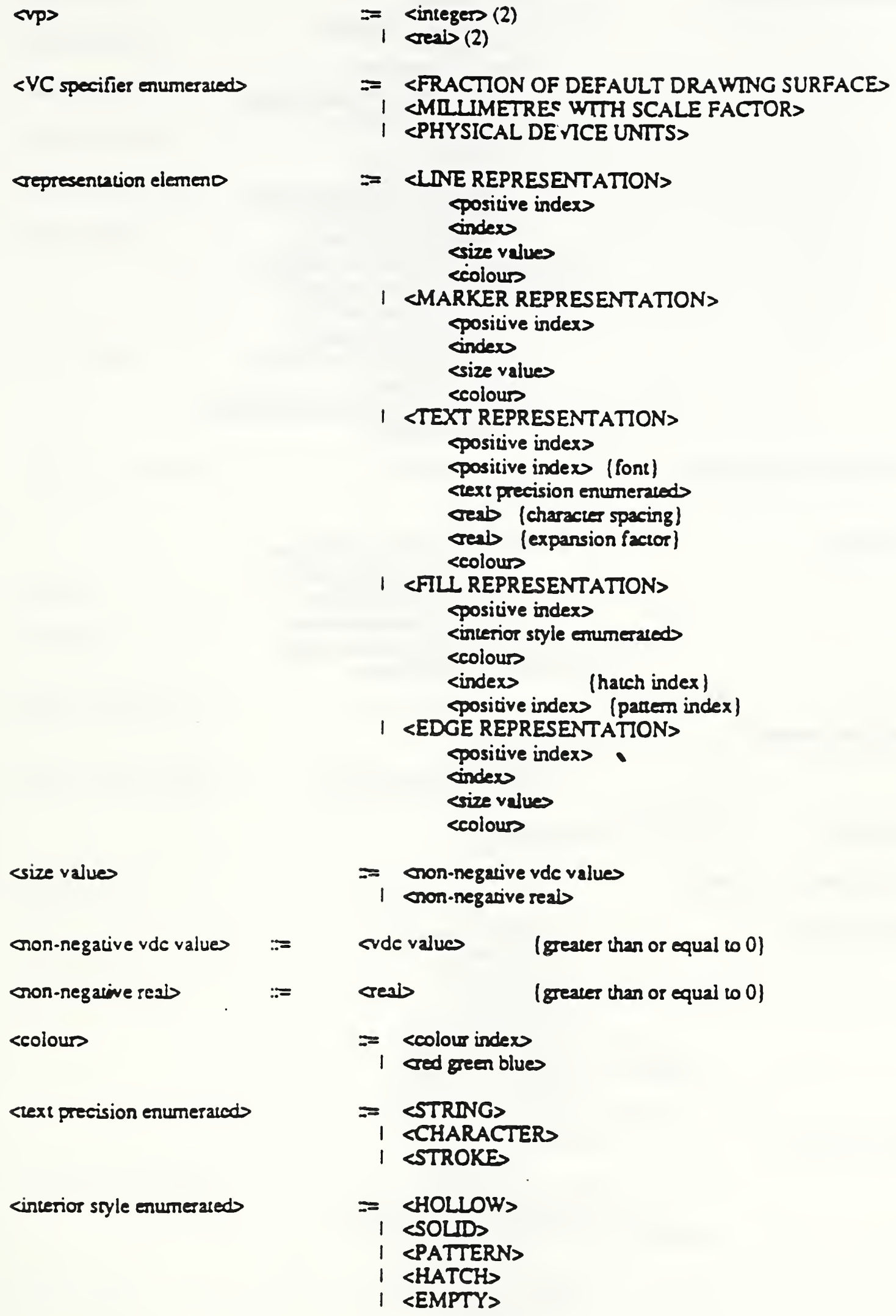


<eligible control elemeno

<on-off indicator enumerated

sude precision

<clip mode enumerateds

<context rames

\section{F.3.5 Graphical elements}

<graphical elemeno

<poiypoint element

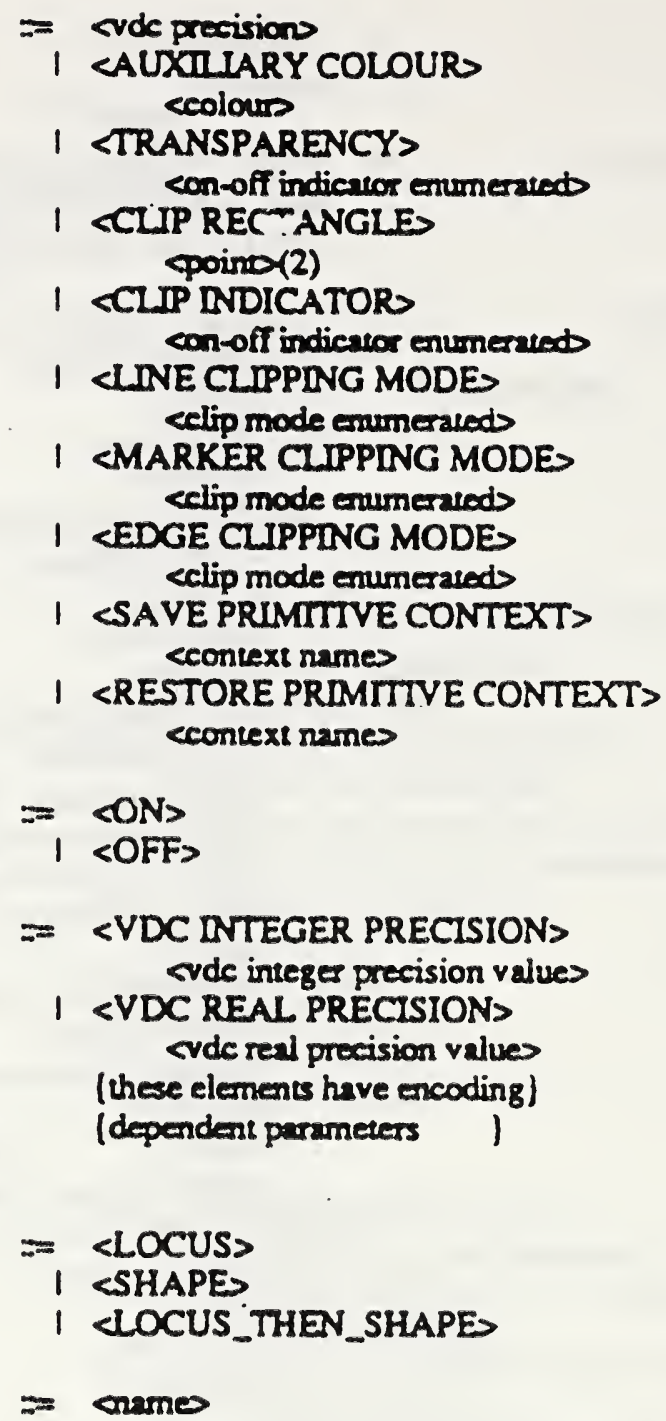




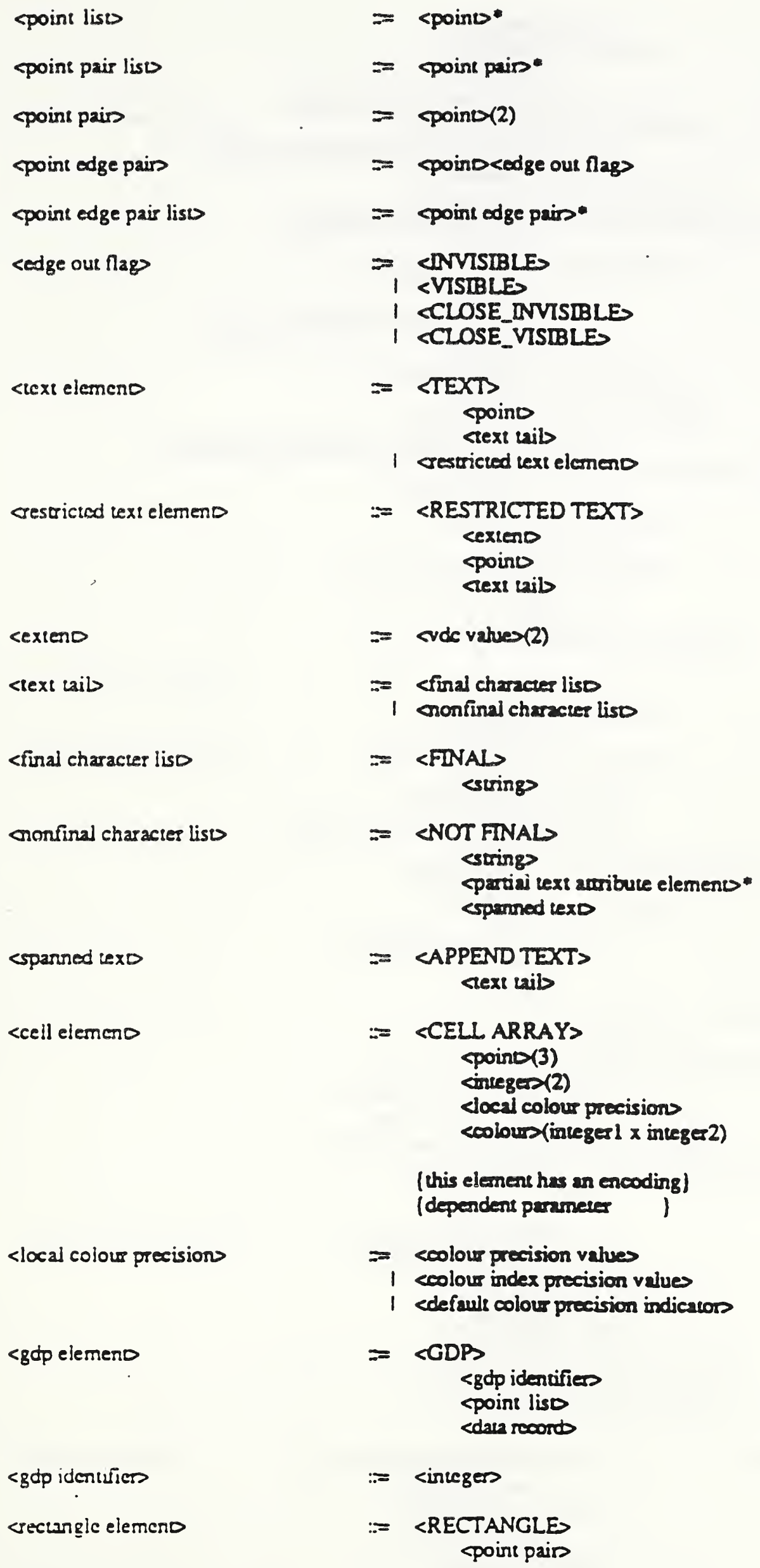


1 CCIRCULAR ARC 3 PODNT Qpoino(3)

I CCIRCULAR ARC 3 PODNT CLOSE> Gointo(3) eclose types

1 CCIRCULAR ARC CENTRE> Spoino ovde vaheo (4) erdiuss

1 CCRCULAR ARC CENTRE CLOSE> spoines sude vahues (4) crdiuss <close types

1 <CIRCULAR ARC CENTRE REVERSED> <poine srde values (4) cadiuss

sadiuss

<close types

<elliprical elemeno

rpoinuless elemend

\section{F.3.6 Attribute elements}

Sprimitive astribute element

cline atribute elemeno

enarker atribute elemeno

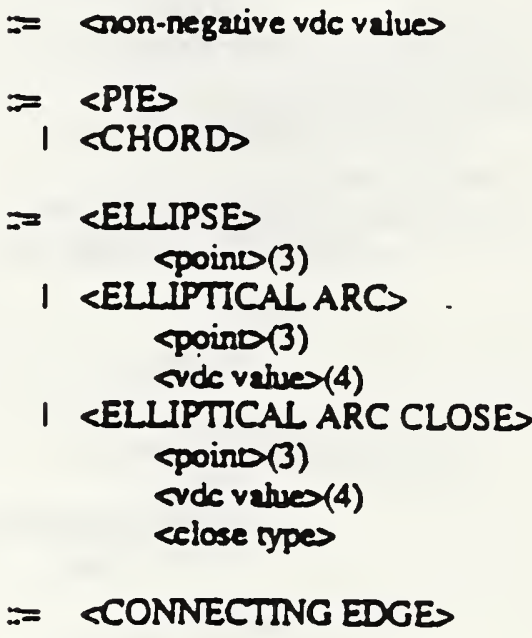

$=$ <line atribute elemeno

I smarke amibute elemeno

I cext atribute elemeno

1 cilled-area anibure elemeno

1 enspect source flagss

I Trick identifies

$=$ <LNE BUNDLE INDEX > Spositive index>

I ILINE TYPES indeso

1 CINE WIDTH crize vahues

1 <LDE COLOUR ecolous

$:=$ <MARKER BUNDLE DNDEX Spositive index>

1 CMARKER TYPES einder

1 <MARKER SIZE 


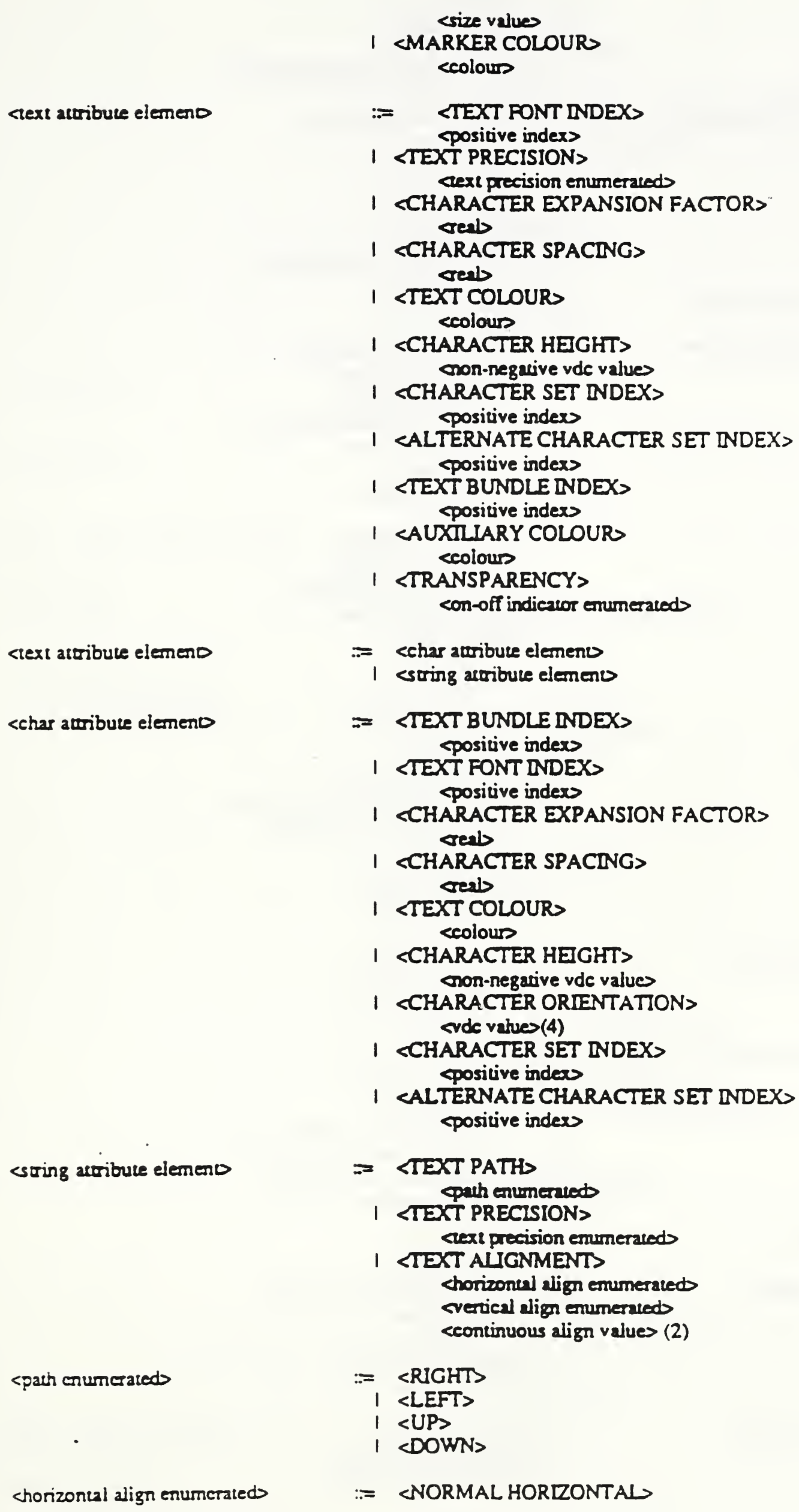$$
:=\text { NORMAL HORIZONTAL }
$$ 
sverical aligs emumerated

<continuous align values

<filled-area atribute elems

<colour table element

Tpartern table elemeno

estaring index>

<2spect source flags>

<3sf pairs

<asf type enumerated
¿LEFT>

CCENTRE

1 <RIGHTS

I CCONTINUOUS HOREONTAL

= NORMAL VERTICAL

1 TTOP

1 <CAP>

1 <HALF>

1 <BASE

1 <BOTTOM>

I CCONTINUOUS VERTICAL .

$=$ sreal

$:=<F I L L$ BUNDLE DNDEX> <positive index>

I CNTERIOR STYLE <interior style enumeratod

1 <FILL COLOUR> <coloun

1 <HATCH INDEX> cinders

| <PATTERN INDEX> Spositive index>

<EDGE BUNDLE INDEX> Qposiuve index>

<EDGE TYPE> sindex>

<EDGE WIDTH> esize values

I <EDGE COLOUR> <colour

1 <EDGE VISIBILTTY> con-olf indicator enumeralod

l <FILL REFERENCE POINT> Tpoino

1 Spastem vable elemeno

1 <PATTERN SLES sude values (4)

$=$ CCOLOUR TABLE estruing index> sed greer bluest

= SPATTERNTABLE Sposigive index> integer (2) <local colour precision <colours (inieger! $x$ integer2)

(this elemeat has an encoding) (dependent parameter )

$=$ colour index

= CASPECT SOURCE FLAGS> easf pairs-

$:=$ <asf type emumeralcd easf enumerateds

$:=$ <LINE TYPE ASF>

1 <LDVE WTDTH ASF>

1 <LINE COLOUR ASF> 


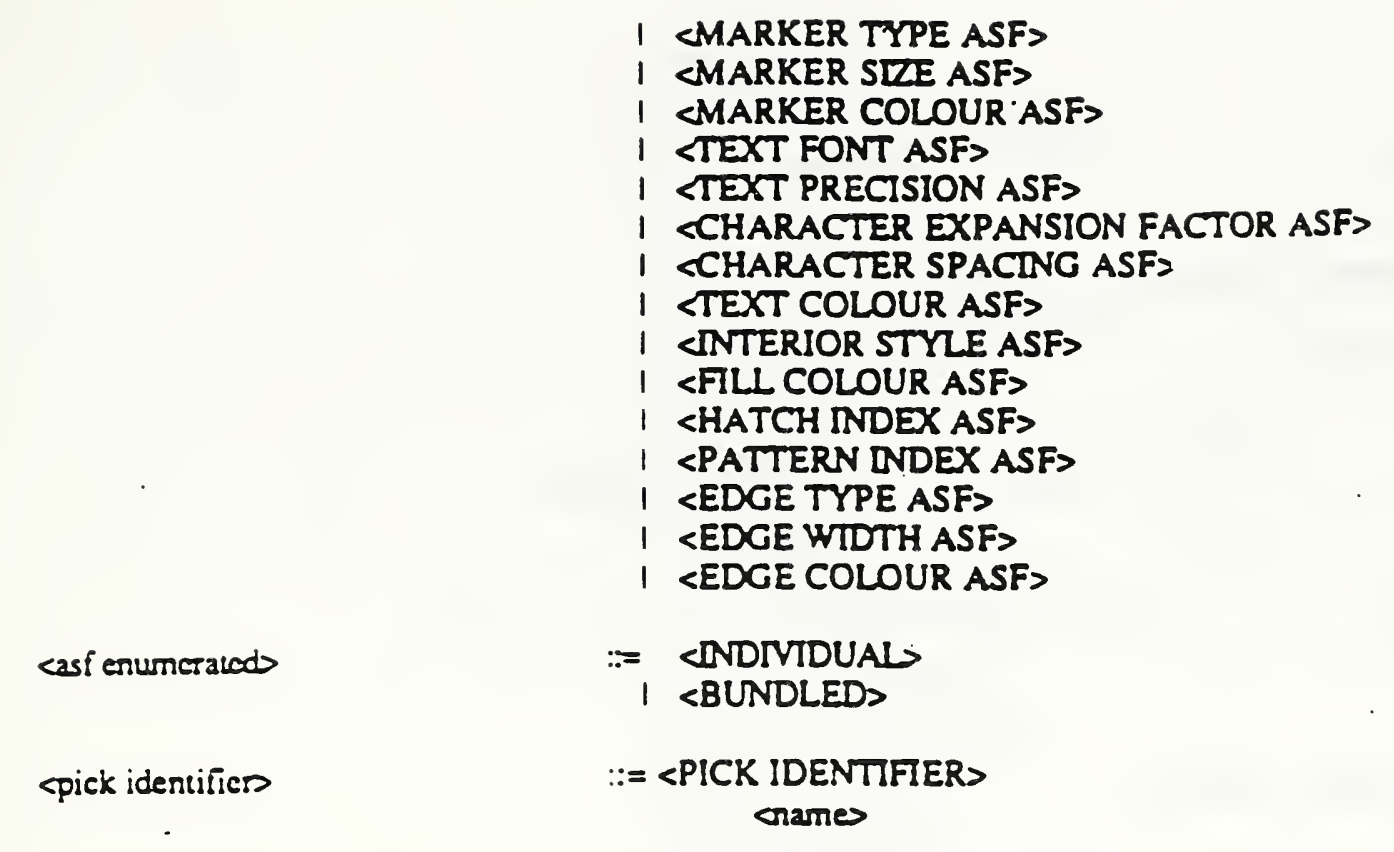

\section{F.3.7 Closed ngure element}

<closed figure
$:=\angle B E G D N$ FIGURE <eligible elements within closed figures> <END FGURE>

<eligible elements within closed figuress

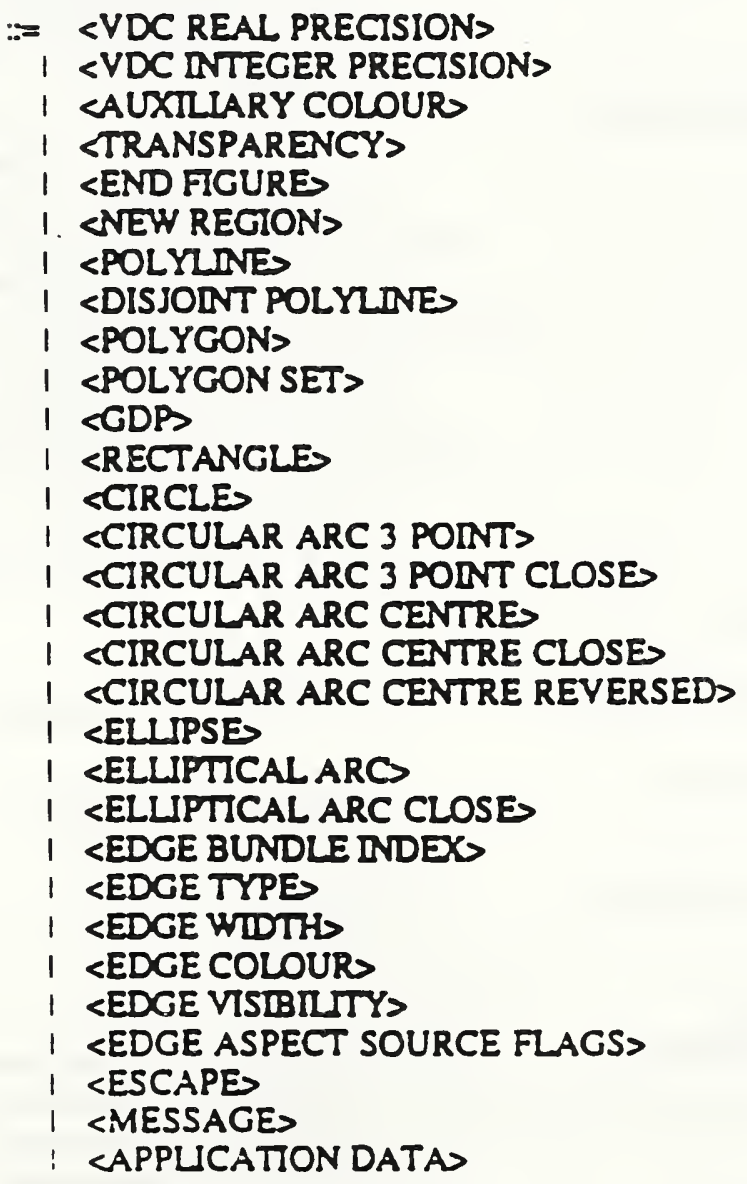

F.3.8 Escape elements 


\begin{tabular}{|c|c|c|}
\hline & & $\begin{array}{l}\text { Sidentifies } \\
\text { edata reconts }\end{array}$ \\
\hline <identifies & $=$ & <integers \\
\hline \multicolumn{3}{|l|}{ F.3.9 External elements } \\
\hline <extemal elemeno & $\begin{array}{r}= \\
1\end{array}$ & $\begin{array}{l}\text { <MESSAGE> } \\
\text { <action lag> } \\
\text { estring> } \\
\text { CAPPLICATION DATA> } \\
\text { <integes } \\
\text { edare recond }\end{array}$ \\
\hline <accion Ilag> & $=$ & $\begin{array}{l}\text { <YES> } \\
\text { SNOP }\end{array}$ \\
\hline
\end{tabular}

\section{F.3.10 Segment elements}

esegment control elemeno

esegment atribure element

esegment identiriess

$:=$ crames

«copy uansformarion marrix

$=$ <transformation matrix>

¿tansformacion marrix>

$=2 \times 2$ marrix of reals 2xl matrix of vdess

esegment tars. application

$=$ NOS

1 <YES>

<ilter selection list enumerateds

$$
\begin{aligned}
& =\text { <auribure and control name enumerated } \\
& 1 \text { <aturibure group and control enumerated } \\
& 1 \text { <ASF name enumerated } \\
& 1 \text { CASF group enumerated }
\end{aligned}
$$

<atribure and control name enumeraced 
1 LINE WIDTH

1 <LINE COLOUR

I <LINE CLIPPDNG MODE

1 SMARKER BUNDLE INDEX

1 <MARKER TYPE>

1 <MARKER SLZE>

I ¿MARKER COL JUR >

1 <MARKER CLIPPING MODE>

1 <TEXT BUNDLE DNDEX>

1 <TEXT FONT DNDEX>

1 <TEXT PRECISION>

1 CCHARACTER EXPANSION FACTOR>

1 CCHARACTER SPACDNG>

1 <TEXT COLOUR

1 <CHARACTER HEIGHT>

I <CHARACTER ORIENTATION>

1 <TEXT PATH>

1 <TEXT ALGNMENT>

1 <FLL BUNDLE DNDEX>

1 ENTERIOR STYLE

1 <FILL COLOUR>

1 <HATCH INDEX>

1 <EDGE BUNDLE INDEX>

1 <EDGE TYPE

1 <EDGE WTDTH>

1 <EDGE COLOUR>

I <EDGE VISIBIITY>

$1<E D G E$ CLIPPING MODE

1 <FILL REFERENCE POINT>

1 <PATTERN TABLE>

1 <PATTERN SEES

I CAUXTIARY COLOUR

1 <TRANSPARENCY>

<acribule group enumeraled>

<eting enumerated

<asf name enumeraied

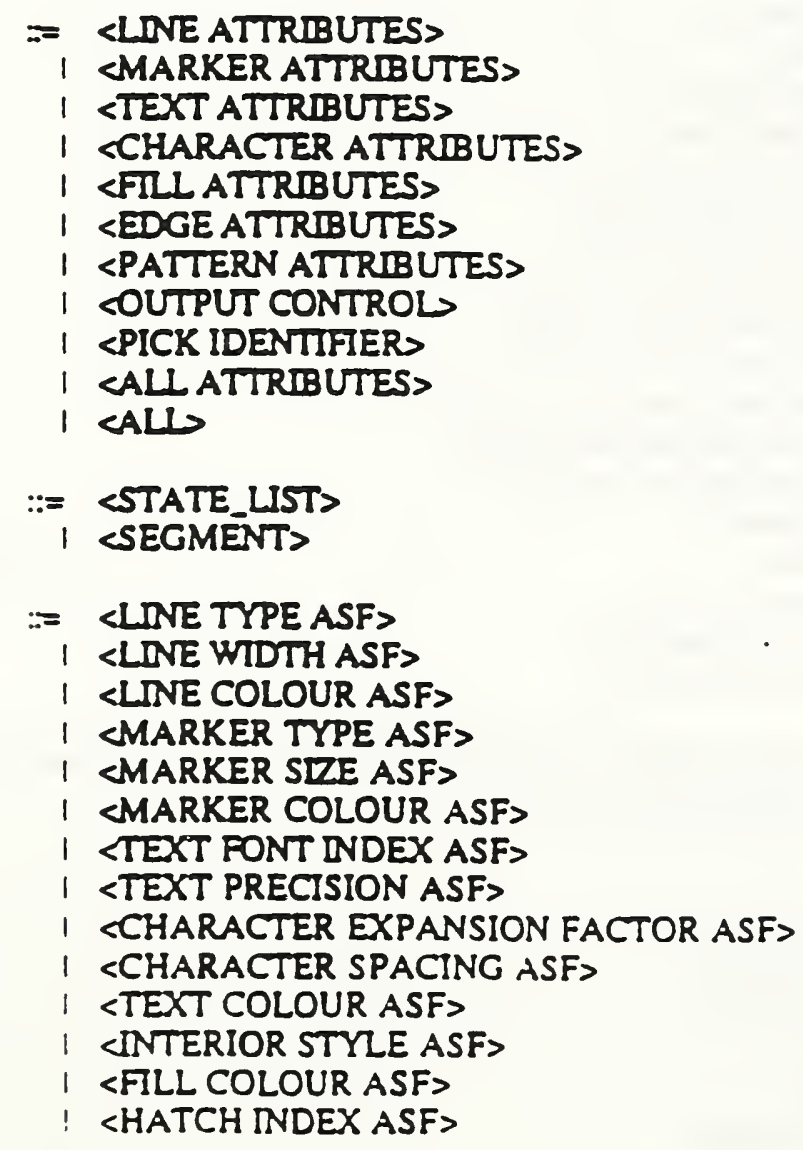




\begin{tabular}{|c|c|c|}
\hline & $\begin{array}{l}1 \\
1 \\
1 \\
1\end{array}$ & $\begin{array}{l}\text { <PATTERN DNDEX ASF> } \\
\text { <EDGE TYPE ASF> } \\
\text { <EDGE WIDTH ASF> } \\
\text { <EDGE COLOUR ASF> }\end{array}$ \\
\hline <asf group cnumerated & $\begin{array}{r}= \\
1 \\
1 \\
1 \\
1\end{array}$ & $\begin{array}{l}\text { <LINE ASFs> } \\
\text { <MARKER ASFS> } \\
\text { <TEXT ASFS> } \\
\text { <FILL ASFS> } \\
\text { <EDGE ASFS> } \\
\text { <ALL ASFS> }\end{array}$ \\
\hline <elip inheritance enumerated > & $=$ & $\begin{array}{l}\text { <STATE_LIST> } \\
\text { CNTTERSECTION> }\end{array}$ \\
\hline <highlighting enumeraceds & $:=$ & $\begin{array}{l}\text { \&NORMAL } \\
\text { <HGHLIGHTED> }\end{array}$ \\
\hline <segment display priority> & \multicolumn{2}{|c|}{$::=\langle$ integer $\rangle$} \\
\hline <segment pick priority> & \multicolumn{2}{|c|}{$::=\langle$ integer $\rangle$} \\
\hline
\end{tabular}

\section{F.4 Terminal symbols}

The following are the termina's in this grammar.

Their representation is dependent on the encoding scheme usod.

In annex $A$ of the subsequent parts of this Standard these

encoding-dependent symbols are furcher described.

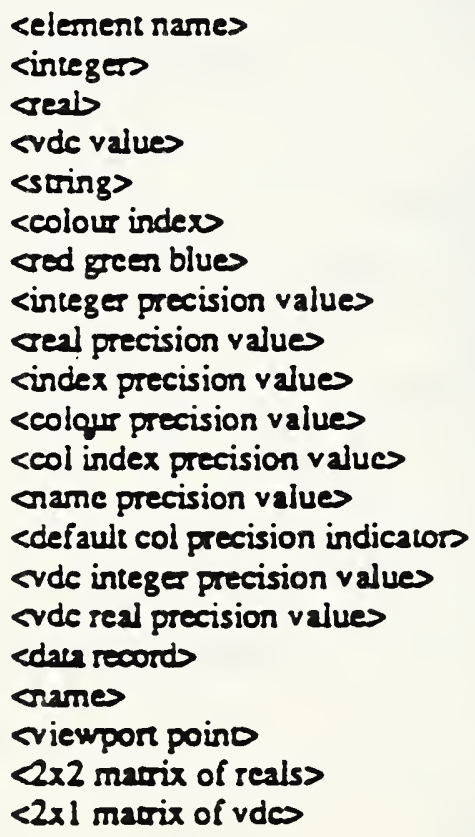

The CGM extended opeodes are encoding dependent. A compiete list of them can be found in the productions for <element name enumerated below.

The crumerated types:

<DTEGER>

$\langle$ REAL $>$

$\langle O N\rangle$

$\langle O F F\rangle$

CDDEXED 


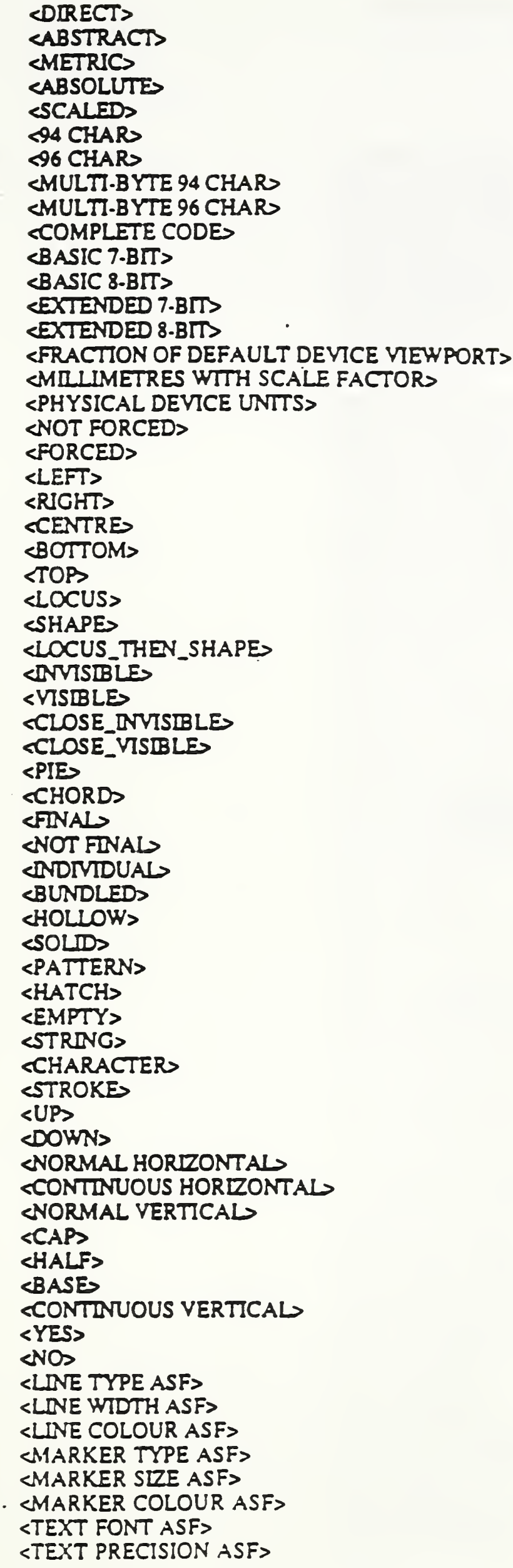


¿CHARACTER EXPANSION FACTOR ASF>

CCHARACTER SPACING ASF>

<TEXT COLOUR ASF>

QINTERIOR STYLE ASF>

〈HATCH DNDEX ASF>

¿PATTERN INDEX $A^{\circ 5}>$

SFILL COLOUR ASF>

¿EDGE TYPE ASF>

¿EDGE WIDTH ASF>

¿EDGE COLOUR ASF>

<LNE ATTRIBUTES>

CMARKER ATTRIBUTES>

<TEXT ATTRLBUTES>

<CHARACTER ATTRIBUTES>

<FILL ATTRIBUTES>

<EDGE ATTRIBUTES>

<PATTERN ATTRIBUTES>

COUTPUT CONTROL

CALL ATTRIBUTES>

CALL

<LINE BUNDLE DNDEX >

<LINE TYPE>

LLNE WIDTH

LLINE COLOUR>

<LINE CLIPPING MODE>

SMLARKER BUNDLE INDEX $>$

CMARKER TYPE>

CMARKE: :I.E

CMARKER COLOUR>

¿MARKER CLIPPDNG MODE

<TEXT BUNDLE INDEX>

<TEXT FONT DNDEX>

<TEXT PRECISION>

<CHARACTER EXPANSION FACTOR>

CHARACTER SPACDNG>

STEXT COLOUR>

SCHARACTER HETGHT>

<CHARACTER ORIENTATION>

<TEXT PATH>

<TEXT ALIGNMENT>

<CHARACTER SET INDEX>

CALTERNATE CHARACTER SET INDEX>

$<$ FILL BUNDLE INDEX>

¿INTERIOR STYLE>

CFIL COLOUR

$<$ HATCH DNDEX>

$\triangle$ PATTERN INDEX>

<EDGE BUNDLE INDEX>

<EDGE TYPE

¿EDGE WIDTH>

<EDGE COLOUR >

¿EDGE VISIBILTY>

<EDGE CUPPDNG MODE

<FILL REFERENCE PODNT>

<PATTERN SLE >

CAUXILIARY COLOUR

〈TRANSPARENCY>

<STATE_LIST>

CATERSECTION>

<SEGMENT>

<LINE ASFS>

- 〈MARKER ASFS>

〈TEXT ASFS>

<FILL ASFS > 
<EDGE ASFS>

¿ALL ASFS>

SNORMAL

CHIGHLIGHTEDS

<element name enumeraied

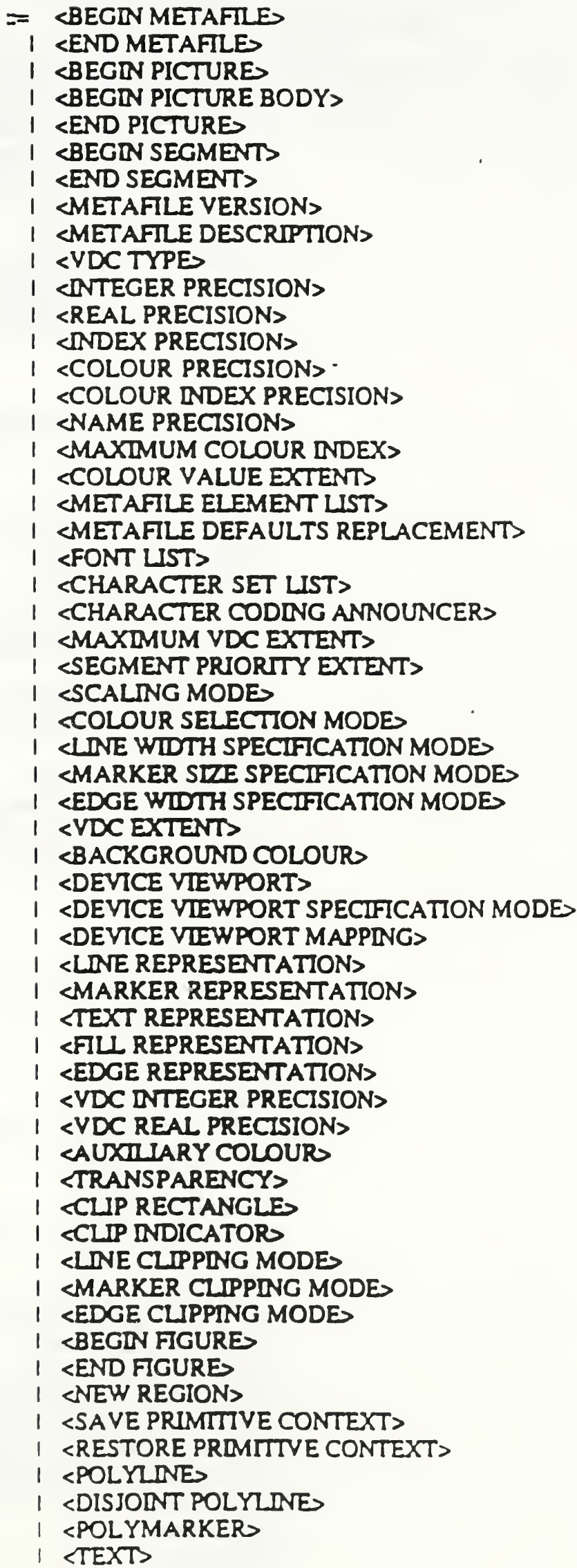




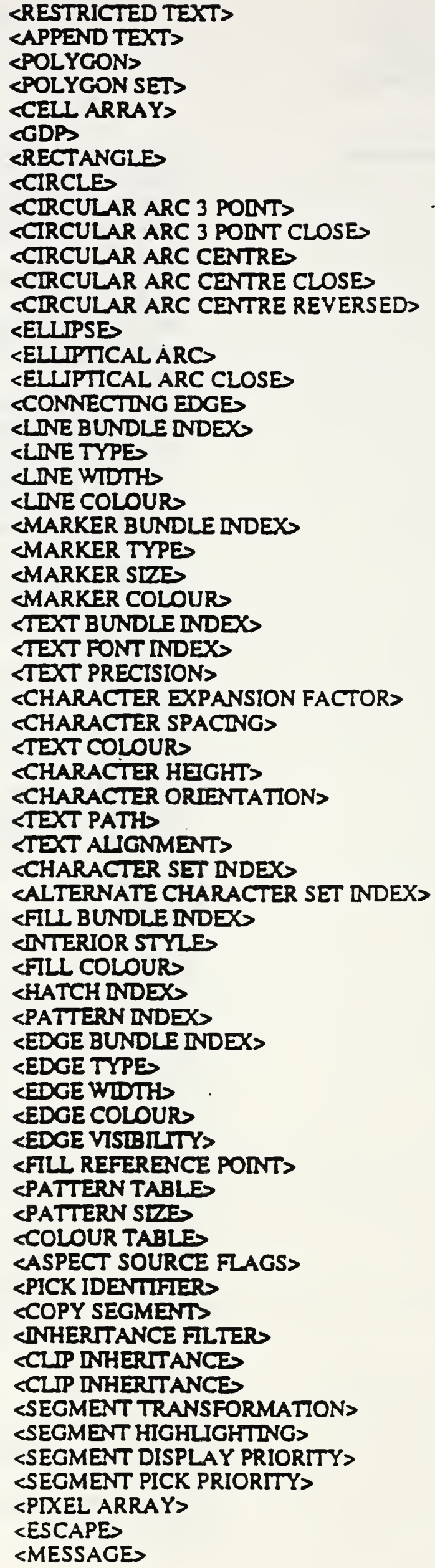




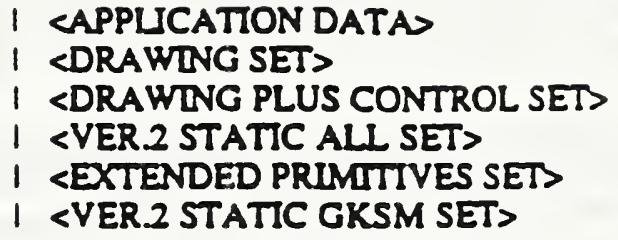




\section{Annex G Relationship of CGM and GKS}

(This annex does not form a part of the standard)

\section{G.1 Introduction}

The GKS Standard includes the concepts of metarile input and output workstations as well is functions providing access $t 0$ and interprevation of metariles. It does noh howeve, contain a metafile definition as part of the Sundard. Annex $E$ of this standard provides a mapping to version 1 metariles.

This Arrex provides a mapping between GKS and the version 2 metriles.

\section{G.2 Scope}

The CGM Add. 2 captures static picture definitions. GKS provides many possibilities 20 generate images. This means that the strategies for generating picnure definitions are numerous and complex. The best strategy 10 use in given cireunitances is dictured by implementation and application requirements. This annex presents a delailed mappings besween GKS and CGM only for one parcicular strategy.

The scope of this annex is further linited to generation of metafiles by GKS and incerpretation of GKS generated metariles in GKS environments. There are many other scenarios for generution and interpretacion of metafiles, such as interpretution by GKS of metafiles not generated by GKS and interpretation by non-GKS processes of GKS generated melasiles. These scenarios are not dealt with in this amex. The amex $C$ presents context models dealing with such cases.

\section{G.3 Overview of the Differences Between GKS and CGM Version 2}

While CGM suppors all of the basic output functionality of GKS. a one-to-one mapping berween GKS and CGM is not possible in all cases mainly because some CGM elements have no coumterpars as GKS functions and some GKS functions have no corresponding CGM element. Examples of this are:

1. Delimiter element like BEGIN PICTURE

2. Enhanced facilities for tailoring and controlling the interpretation of the metafile precision of various items, and the control of default values.

3. Extended capabilities in the area of text processing, such as named font, changing character sets and restricted lext.

\section{G.t Mapping Concepts}

The cables laser in this annex presens mappings between GKS and CGM elements.

\section{G.4.1 Principles}

The following principles are basis of the GKS/CGM model of this amex and of the function mappings themselves:

a) conceprual compaibility with GKS

b) compatibility with the design concepts of CGM

\section{G.4.2 Workstation}

The CGM is generued in this model, by a workstation of type MO. The behaviour of the workestation, particularly in response to dynamic GKS functions, can be illustrated by analog: in most respects, the MO/CGM worksution in GKS may be implemented in a manes analogous to a workstation of category OUTPUT (e g., a plotter), whose device instruction set corresponds to the CGM elements. Strategies for correctly sending device instructions to such a real device are similar to those generaing the proper elements on the metarile.

The CGM is read by a worksution of calegory MI. Certain elements, such as the metarile descriptor and precisionseting elements. are viewed as directives to the MI workstation itself, so that it may correctly read the metafile contents.

\section{G.4.3 Picture generation}


A metafile is composed of a collection of murually independent pictures. GKS does not have the concept of "picture" as defined in CGM but it does formalize the notion of an empry view surface. GKS actions which cause clearing of the view surface, such as CLEAR WORKSTATION, are defined to delimit metafile picare. There is anocher mechanism which leads to generation of picurres in this model of the GKS/CGM relationship. GKS conuains functions which have potencial dynanic effects on a non-empry display surface. The CGM design concepts exclude dynumic modification of $p^{\prime}$ :ares. For this reason all "dymamic modification accepted ..." values of \& MO/CGM wortstation will be concepasally IRG.

The default value of the deferral state on an MO/CGM workstation is ASTI-SUPPRESSED.

This model of the MO/CGM workstation defines that whenever a GKS function is invoked which causes a regeneration. then a picrure is output wo the metarle.

\section{G.4.4 Coordinates and clipping}

The coordinate space of the meesfile. VDC, is defined as being idencical to the NDC space of CKS. Clipping and transformation are compiedy deferred to the metarile interpreser. Each GKS elip and ransformation element has a counterpars in CGM.

\section{G.4.5 Workstation transformation}

The workstation transformation is derined in GKS by seaning a workstation window in deviec-independent NDC and a workstation viewport in device-dependens DC. The workstation window is wrinen to the metafile with the VDC EXTENT element The workstation viewport is written to the metafile with the DEVICE VIEWPORT element.

The default values of DEVICE VIEWPORT MAPPING correspond to GKS mapping of the device coordinate systen onso the display space. The DEVICE VIEWPORT SPECIFICATION MODE is set to MILLIMETRES WITH SCALE FACTOR and merric seale factor 1000.0 within the METAFILE DEFAULTS REPLACEMENT element

\section{G.4.7 Metafile element list .}

The metarile element list shor hand defined for use with GKS application is 'version2-static-gksm'.

\section{G.4.8 Relationship of fonts between CGM and GKS}

The GKS standard includes the concepts of text output primitive atrributes. However. the mechanism for specifying the text font differs from that specinied in the CGM standard. This elause defines the approach to handling these atributes within the GKS environment.

\section{G.4.8.1 Overview of the differences between GKS and CGM fonts}

While CGM supports the TEXT output primitive attribute functionality of GKS, a one-w-one mapping berveen CGM and GKS is not possible in all cases. Specifically:

1) GKS and CGM diffe in the way fonts are defined:

In the CGM text fonts are derned with the FONT LIST element that associates font names or identifications with entries is a Font Tabie.

In GKS. no mechanism is available for delining text fonts. GKS associates a trique text font number with each font The Registration Authority is responsible for defining this mapping of font numbers to specific font identifications.

2) GKS and CGM differ in the way fonts are selected.

In the CGM. text fonts are selected with the TEXT FONT DNDEX element The index sciccts an individual font from different fonts in the font list

In GKS. text fonts are selected with a font number. The font number selects a specific GKS registcred font if the value is positive. If the font number is negarive an implementation dependent font is selected.

GKS and CGM differ on the independence of font and text precision: 
In the CGM, the font and text precision are specified by independent elements.

In GKS, the font and lext precision are directly associated with specification by a single function.

4) Some CGM Elements have no councerpart as GKS functions:

These include i ixiliary Colour relued elements, such as AUXIUARY COLOUR and TPANSPARENCY that affect the presentation of texl.

This additional functionality of the CGM causes no special problems for a GKS environment interpreting a version 2 CGM.

5) The character set related elements CHARACTER SET LIST. CHARACTER CODNG ANNOUNCER. CHARACTER SET INDEX. ALTERNATE CHARACTER SET DNDEX have no counierpart in GKS. GKS does not recognize the concept of characier set as a separate concept from the font concepp. GKS implementors are encouraged to provide a mapping to the character set elements for boch MO and MI workstations to increase the possibility of transferring metarile berween GKS environments and other systems.

\section{G.4.8.2 Suggestion for interpretation of CGM font information by GKS}

GKS environments interpreting a CGM specify fonts with a font number. It is assumed that GKS maintains a list associating positive font mumbers with a GKS registered font name or identifier. Private font numbers (i.e. negative values) must be maineained in an implementation dependent list of associations. As the FONT LIST element is interpreted an additional list must be maintained that associutes individual font names specified in the CGM with a font index. When the TEXT FONT DNDEX elemen is interpreted, the font name associzted with the font index is determined from the list of currently used fonts. The font name is used to determine the GKS font number associated with this font from a list of GKS registered fonts. This font number is used as the font parameler of the TEXT FONT AND PRECISION function. The value of the precision parameter is taken from the TEXT PRECISION eiement

\section{G.4.8.3 Generating CGM font information from GKS}

When generating font information from GKS vis TEXT FONT AND PRECISION it is recommended that the generzior also writes the elements CHARACTER SET DNDEX and ALTERNATE CHARACTER SET INDEX as well as TEXT FONT DNDEX and TEXT PRECISION. The generawor is assumed to have a uable associating the positive font numbers of GKS with the registered names. The generuor shall pur a FONT LIST element in the Metarile Descripxor with the names of those fones referenced by positive GKS font numbers. Negarive GKS font numbers are privale and must be mapped to CGM font indices which are positive and beyond the range of the table."

NOTE: The metarile must be completely generated before the FONT UST element can be writuen.

\section{G.5 Metafile Generation}

Included in following tables is a particular set of mappings of the GKS function, workstation state list entries and segment state list entries onto CGM elements. The mupings presented are deemed usable and suitable for guiding implementation of a CGM picrure generator in a GKS environment. The mapping concepts of G.4 are assumed.

\section{G.5.1 Control functions}

OPEN MORKSTATION

CLOSE WORKSTATION

ACIIVATE HORKSTATION
BEGIN METAFIIE

(Metafile Descriptor)

BEGIN PICTURE

store current workstation state list

BEGIN PICTURE BODY

END PICTURE

END METAEIIE

attribute settings

CIIP RECTANGIE 
CIIP INDICATOR

enable output to metafile

DEACTIVATE WORKSTATION

CIEAR WORKSTATION

control flag = CONDITIONAL

display space empty = EMPTY

CIEAR WORKSTATION

display space empey = NOTEMPTY disable output to metafile

no Action

END RICTURE

BEGIN PICTURE

store current workstation state list

BEGIN PICTURE BODY

attribute settings

CIIP RECTANGLE

CIIP INDICATOR
(3)

attribute settings

CIIP RECTANGLE

CIIP INDICATOR

generate all visible segments stored for

the Mo workstation
URDATE WORKSTATION

regeneration flag = PERFORM

new frame action necessary

at update $=$ YES

URDATE WORKSTATION

regeneration flag = PERFORM

new Erame action necessary

at update $=$ NO

or

URDATE WORKSTATION

regeneration Glag = POSTPONE

SET DEFEMRAL STATE

new frame action necessary

at updare $=$ YES

ESCAPE

MESSAGE
AS REDRAH AII SEGMNTS ON WORKSTATION no Action

as REDRAN AII SEGMENTS ON WORKSTATION

ESCAPE

MESSAGE

NOTES

1) The use of the identifier parameter in BEGIN METAFILE is implemenation dependent.

2) Sce G.5.5 Metarile Descriptor

3) The use of the identifier parameter in BEGIN PICTURE is implementation dependent.

4) Sic G.5.6 mapping of workstation state list to CGM element

5) The uttributc scttings ensure that the metafile attributes in effect when the first graphical primitive clement of a picture is encountered match the current attributes from the GKS state list.

6) Gincrutcd sequence of CGM-Elements for every segment as ASSOCIATE SEGMENT WITH IVORKSTATION (see G.5.3.4) 


\section{G.5.2 GKS function leading to an implicit regeneration}

Depending on the deferal state the following GKS functions may act as REDRAW ALL SEGMENTS ON WORKSTATION because concepaully all corresponding "dymamic modification accepred ..." entries in the workstarion description uable are ses w IRG (see G.4.3)

SET POLYLNE REPRESENTATION
SET MARKER REPRESENTATION
SET TEXT REPRESENTATION
SET INTERIOR REPRESENTATION
SET PATTERN REPRESENTATION
SET COLOUR REPRESENTATION

SET WORKSTATION WTNDOW

SET WORKSTATION VIEWPORT

SET SEGMENT TRANSFORMATION

SET VISIBITTY

SET HIGHLGHTING

SET SEGMENT PRIORTTY

all primitives added to open segments overlapping segments of higher priority.

DELETE SEGMENT

DELETE SEGMENT FROM WORKSTATION

ASSOCIATE SEGMENT WITH WORKSTATION

G.5.3 GKS function with no direct dynamic effect

\section{G.5.3.1 Mappings of output function}

GKS function

CoM Element

Notes

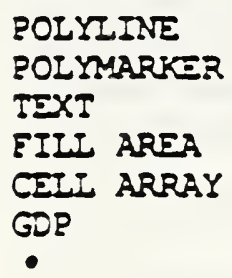

POLYIINE POLMMARKER

TEXT

EIII AREA

CEII ARRAY

GDP

\section{G.5.3.2 Mappings of output attributes}

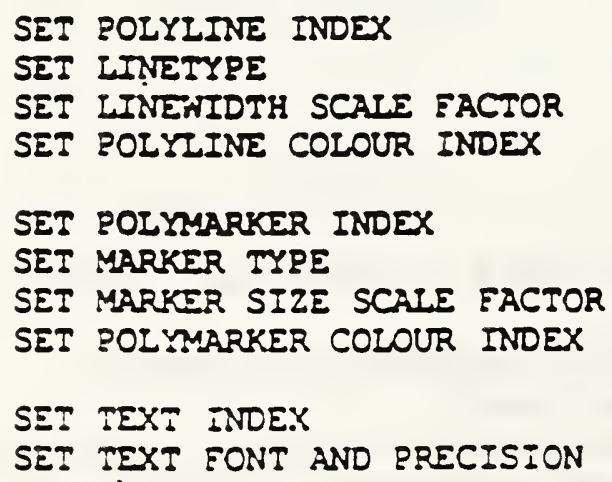

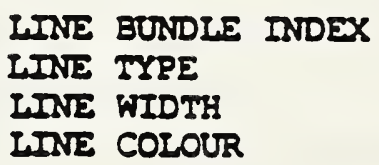

MARKERR BUNDLE INDEX

MARKER TYPE

MARKER SIZE

MARKER COLOUR

TEXT BUNDLE INDEX

TEXT FONT INDEX

TEXT PRECISION

CHARACTER SEI INDEX

ALTERNATE CHARACTER SET INDEX 


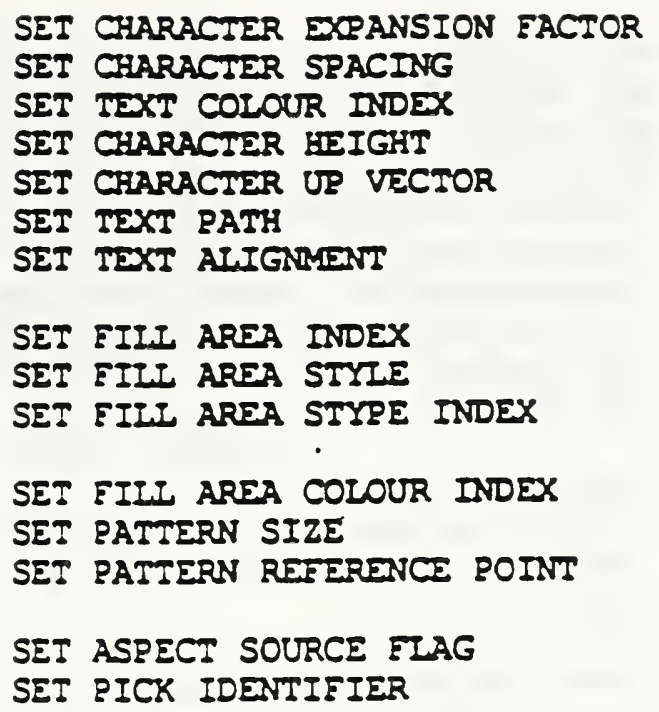

NOTES

1) The default specification modes SCALED apply.

2) The default colour selocrion mode INDEXED applies.

3) GKS includes the notion of character set within 'font', whereas CGM separates the two concepts. When the value of 'font' in the GKS state list changes, then the CGM elements TEXT FONT INDEX, TEXT PRECISION, CHARACTER SET INDEX and ALTERNATE CHARACTER SET INDEX are written to the metafile, each with the value of the 'font' and 'precision' entry in the GKS state list. The CGM font index is determined as described subclause G.4.8.2. The elements shall appear consecutively in the metafile but may appear in any order.

4) Legal values of the GKS 'ill area style index' differ depending upon whether the current interior style is 'hatch' or 'pattern'. Therefore a negative GKS style index results only on the generation of the HATCH DNEX element, and a positive value results in the generation of both the HATCH INDEX and PATIERN INDEX elements.

\section{G.5.3.3 Mappings of transformation function}

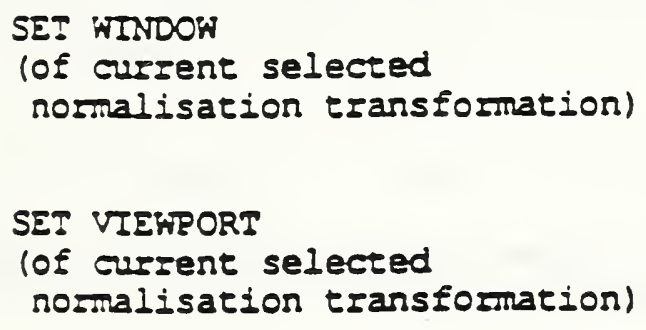

SET NORMAIISATION TRANSFORMATION

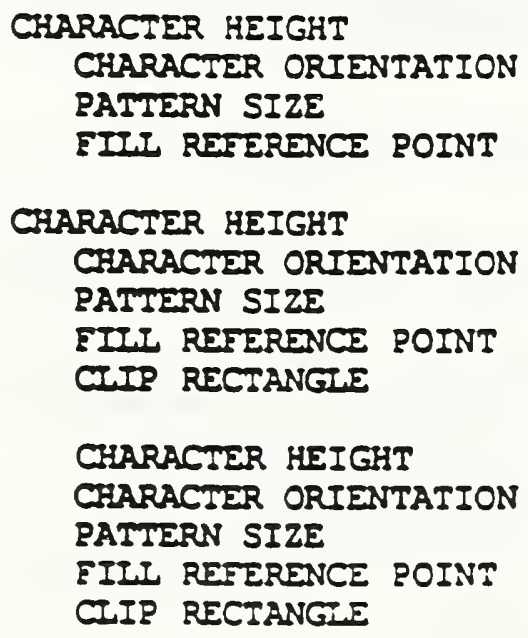

\section{G.5.3.t Mappings of segment manipulation function}


CREATE SEGMENT

CLOSE SEGIENT

RENAME SEGMENT

ASSOCIATE SEGMENT WITH WORKSTATION

COPY SEGENT TO WORKSTATION

INSERT SEGMENT TO WORKSTATION

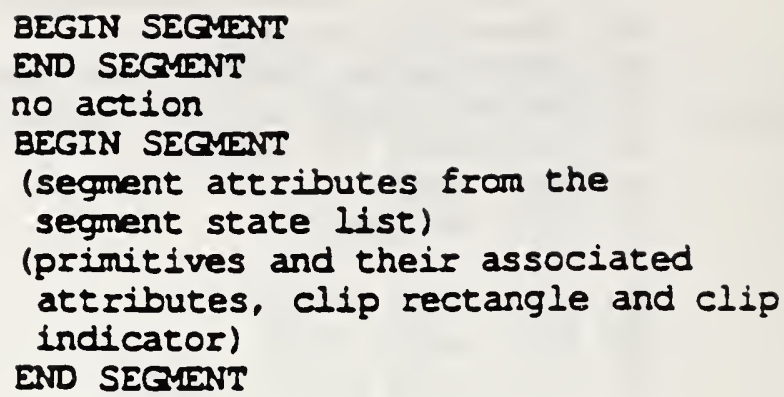

(transformed

primitives and their associated attributes, clip rectangle and clip indicator)

(transformed primitives and associated attributes)

NOTES

1) Primitives transformed by the segment tansformation.

2) Primitives transformed by the segment transformation followed by the inser transformation.

\section{G.5.3.5 Mapping; of segment attributes}

see G.5.2.4, G.S.4 and G.5.7

\section{G.5.t GKS function with no Action

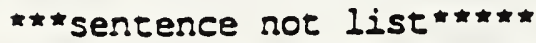 \\ GKS function}

Nores

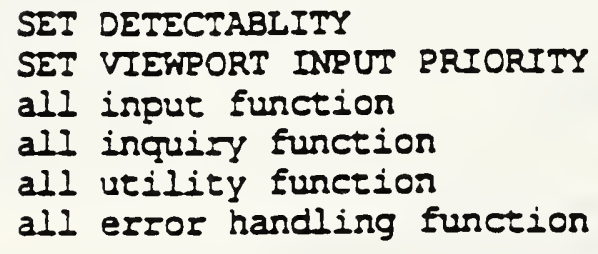

\section{G.5.5 Metafile Description}

At the head of a metarite is a set of Metarile Descriptor (MD) elements. It is useful to view these elements as forming a Metarile Description Table (similar to the GKS and Workstation Description Table in GKS).

In the GKS context, the following description table would be written at the beginning of a metafile. For the elements which are listed 25 "i.d", it is implementation dependens both whether the elements are included in the table and wha values are assigned to the elements.

Metafile element list

Element Value

Mandatory

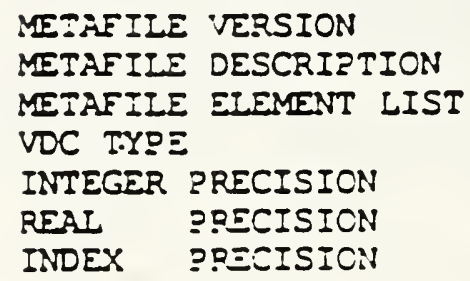

i.d.

i.d.

i.d.

i.d.

i.d. 
COLOUR RRECISION

COLOUR INDEX PRECISION

MAXIMUM VDC EXTENT

MAXINTM NPC EXTENT

COLOUR VALUE EXTENT

SEGIENT PRTORTTY EXTENT

METAFIIE DEFAUIT REPLACEMENT

FONT LIST

CHARACTER CODING ANNOUNCER

CHARACTER SET LIST

global segments i.d.

i.d.

i.d.

i.d.

i.d.

i.d.

i.d.

i.d.

i.d.

i.d.

i.d.

i.d. = implementation dependent

METAFILE VERSION. METAFILE CATEGORY and METAFILE ELEMENT LIST are mandaLry. All metafile defaults sasisfy the GKS Description Table. Inclusion of the METAFILE DEFAULTS REPLACEMENT element change any control. picrure descriptor. and attributc defauls is optional and implentation dependent. It is also implementation dependent whether the CG.M generator includes any of the other MD elements. such as the precision seting elements.

\section{G.5.6 Mapping of workstation state list entries to CGM elements \\ GKS workstation state list entry \\ cQM element}

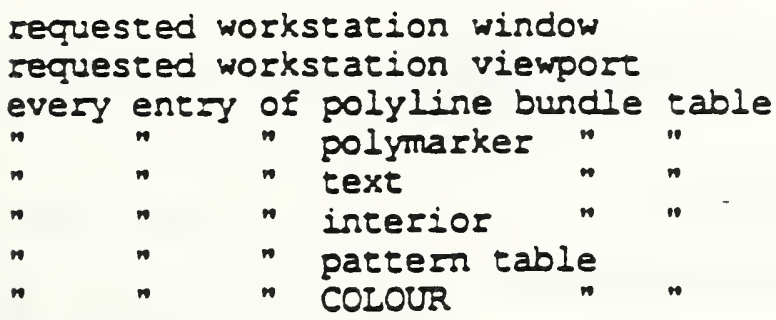

NDC EXTENT
DEVICE VIEAPORT
LINE REPRESENTATION
MARKER "
TEXT " "
FIII
PAITERN TABLE
COLOUR "

NOTES

1) The position of the workstation window within the NDC unit square corresponds to position of the VDC extent withir the maximum VDC exterst.

2) DEVICE VIEWPORT SPECIFICATION MODE and DEVICE VIEWPORT MAPPING may be specificd only within METAFILE DEFAULTS REPLACEMENT in the metafile descripror. The VSU specifict may be either 'millimetres with seale facior' with metric seale factor 1000.0, or 'physical device uniss'.

\section{G.5.7 Mapping of segment state list entries to CGM Add.2 elements GRS segment state list entry \\ cal element}

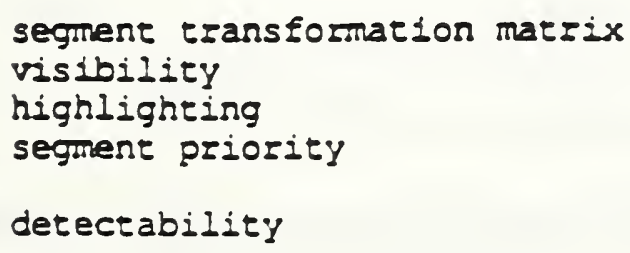

NOTES

1) invisible segmenis are not mapped.

2) The clemenes shall appeas consccutively in the metafile but may appear in any order.

G.5.8 Mapping of metafile function 
NOTES

1) The GKS item type is mapped to the CGM identifier.

\section{G.6 Metafile Interpretation}

\section{G.6.1 Introduction}

This sub-ciause describes how metafile eicments from a version 2 metafile generated by a GKS program according to the mapping described in sub-clause G.S, are subsequenly interpreted by the GKS INTERPRET ITEM function and/or the MICGM workstation. Other guidlines for interpretation are posible.

Those CGM elements which do not map to a GKS item are viewed as directives to the MI/CGM workstation itself. so that it may correctly read the metafile conteris.

A mumber of the elements below are specified as causing GKS state list entries to be set and have parameters specified in VDC (which corresponds to GKS NDC). The GKS state list eneries are in WC. The VDC (NDC) are mapped by the inverse of the current normalization transformation before the GKS state list values are set. The table also ixcludes ilem types wo be resumed wo GKS. These are adopled from GKS Annex E.

\section{G.6.2 Mapping of delimiter elements}

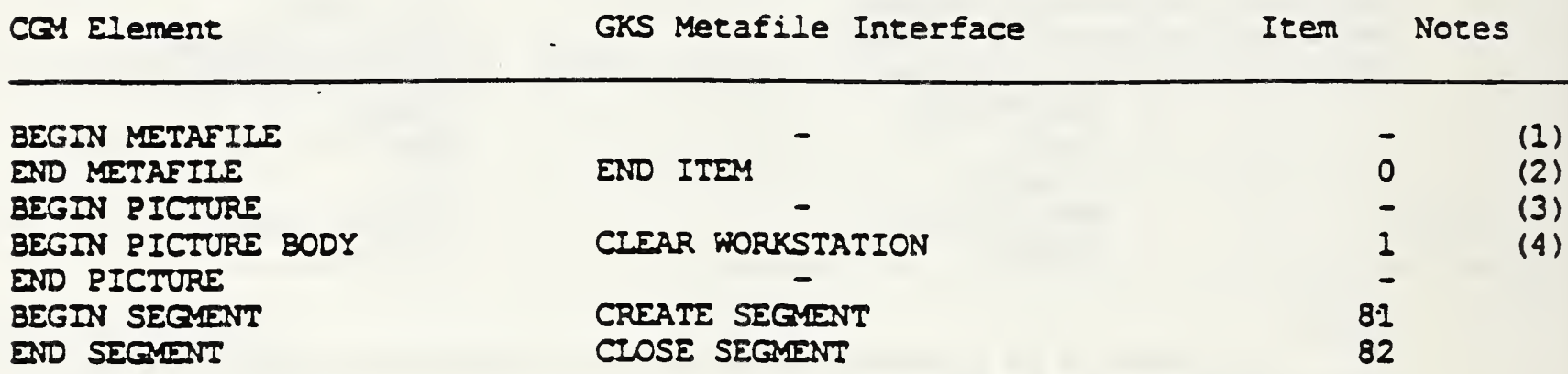

\section{NOTES}

1) The first CGM element interpreted by the MI workstation. The metafile description table inmediately follows. Its elements inform the MI workstation how to read the metafile.

2) No furcher ilems may be read.

3) Appropriate GKS state list values are set to correspond to CGM defaults. Appropriate workstation state list values on active OUTPUT and OUTIN workstations are set 10 correspond to CGM defaults. It is not intended that this action, or the ineerpretation of any picture descriptor elements. cause any immediate dynamic changes to the view surface, which is cleared upon BEGLN PICTURE BODY - the implementation may wish to buffer these actions to suppress such changes, if such changes are undesirable. Onily pienure descriptor eiements may be inuerpreted unil BEGN PICTURE BODY.

4) Causes a CLEAR WORKSTATION on all active worksuations.

\section{G.6.3 Mapping of metafile descriptor elements}

All elements in this class contrin only directives to the MI workstation. their interpretation does not correspond to the invocation of any GKS function.

$\begin{array}{ll}- & - \\ - & -\end{array}$


DNTEGER PRECISION

REAL PRECISION

INDEXX PRECISION

COLOUR PRECISION

COLOUR INDEX PRECISION

MAXIMUM COLOUR INDEX

COLOUR VAIUUE EXTE \&?

METAFIIE ETEMENT IIST

METAFIIE DEFAULTS REPIACEMENT

FONT LIST

CHARACTER SET LIST

CHARACTER CODING ANNOUNCER

MAXIMUM VDC EXTENT

MAXIMUM NPC EXTENT

SEGMENT PRIORITY EXIEENT

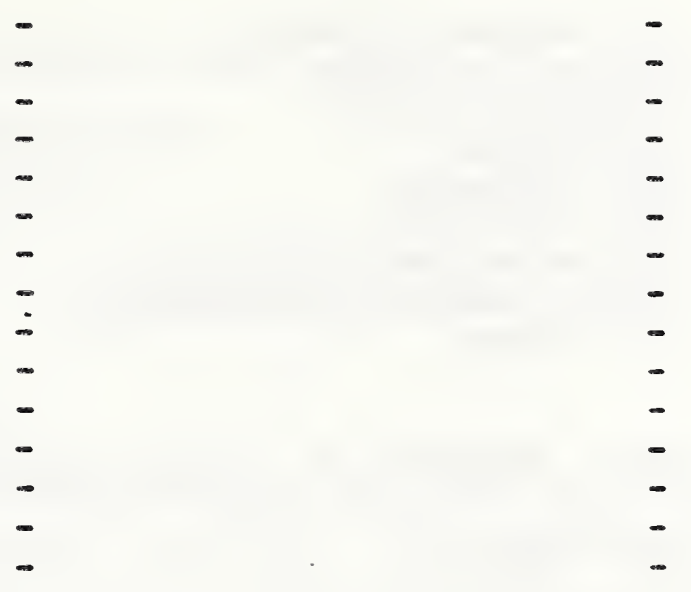

NOTES:

1) The value of the parameter must be 2

2) Used to normalize colour direct values to the continuous range of real numbers $[0,1]$.

3) Used to nomalize VDC range (i.e.NDC) and applics to VDC type INTECER or REAL

4) Used to normalize NPC range to allow the proper position of the NPC EXTENT (workstation window) within the normalized NPC range (the unit square).

5) Used to normalize segment priority to the continuous range of real numbers $[0.1]$.

\section{G.6.4 Mapping of picture descriptor elements}

\begin{tabular}{|c|c|c|c|}
\hline CQM Element & GRS Metafile Interface & Item & Notes \\
\hline COLOUR SEIECTION MODE & - & & - \\
\hline BACKGROUND COLOUR & - & & - \\
\hline DEVICE VIEHPORT & WORKSTATION VIEHPORT & 72 . & \\
\hline DEVICE VIEHPORT & & & \\
\hline SEECIFICATION MODE & - & & (1) \\
\hline DEVICE VIEAPORT MAPPING & - & & 12 \\
\hline LDNE REPRESENTATION & POLYIIINE REPRESENTATION & 51 & 1 \\
\hline MARKER REPRESENTATION & POLMMARKER REPRESENTION & 52 & 2 \\
\hline TEXI RSPRSSENTATION & TEXT REPRESENTATION & 53 & 3 \\
\hline FIII REPRESENTATION & EIII AREA REPRESENTATION & 54 & 4 \\
\hline PATLDRN TABTE & PATTERN REPRESENTATION & 56 & 6 \\
\hline COLOUR TABIS & COLOUR REPRESENTATION & 57 & 7 \\
\hline
\end{tabular}

NOTES:

1) The VSU specifier may be either MILLIMEIRES WITH SCALE FACTOR with metric scale factor equal to 1000.0, or PHYSICAL DEVICE UNITS. DEVICE VIEWPORT SPECIFICATION MODE may occur only within METAFILE DEFAULTS REPLACEMENT.

2) The isotropy nag must be FORCED and the alignment nags must be LEFT and BOTTOM. DEVICE VIEWPORT MAPPING may occur only within METAFILE DEFAULTS REPLACEMENT.

G.6.5. Mapping of control elements

CGY Element GKS Metafile Interface Item Notes 
VDC INTEGER PRECISION

VDC REAI PRECISION

AUXIIIARY COLOUR

TRANSPARENCY

CIIP RECTANGLE

CLIP INTTCATOR

SAVE PRTMITIVE ATIRIBUTES 3

RESTORE PRTMITIVE ATIRIBUTES 3

CIIP VOLUNE

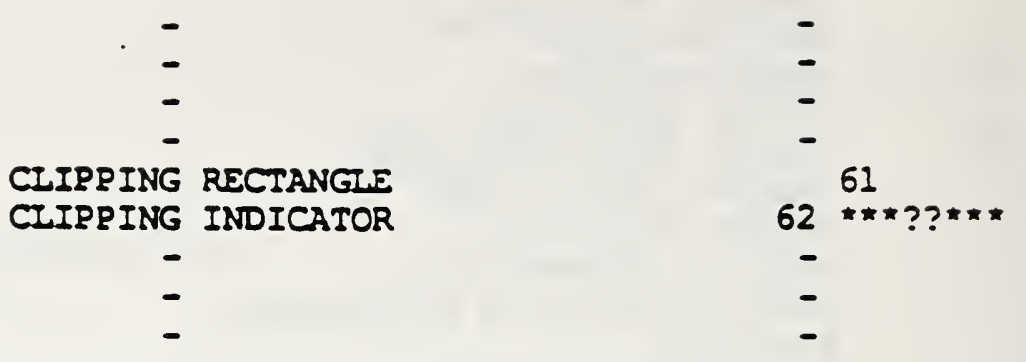

\section{G.6.6 Mapping of graphical primitive elements}

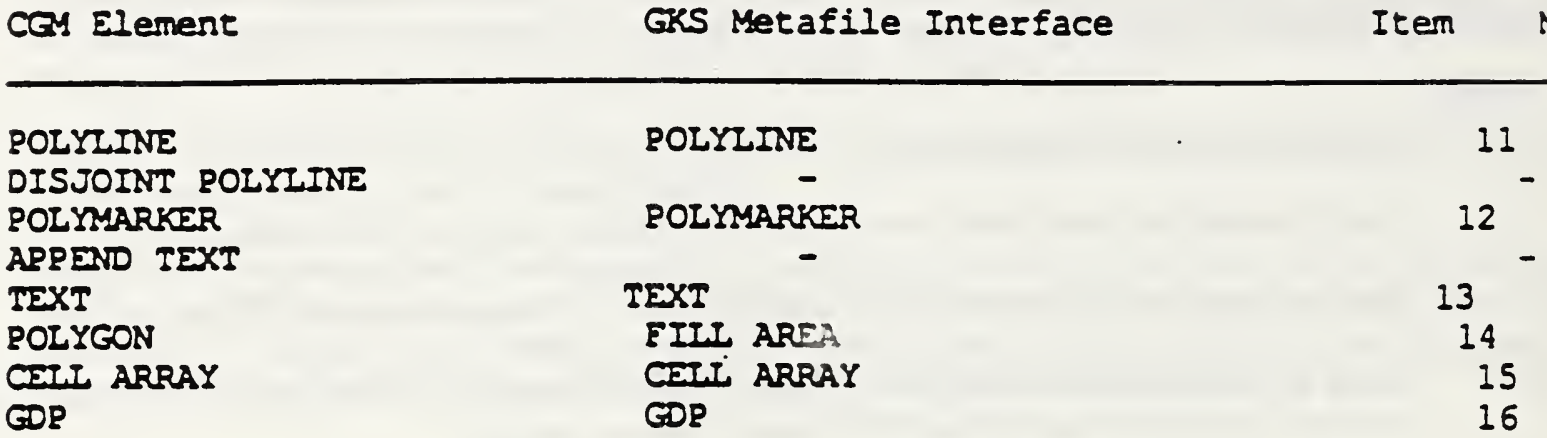

Notes

NOTES

1) The text flag should be 'final'.

\section{G.6.7 Mapping of attribute elements.}

CGY Element

IINE BUNDIE INDEX

LINE TYPE

IINE WIDTH

IINE COIOUR

MARKER BUNDLE INDEX

MARKER TYRE

MARKER SIZE

MARKER COLOUR

TEXT BUNDLE INDEX

TEXT FONT INDEX

TEXT PRECISION

CIARACTER EXPANSION FACTOR

CHARACTER SPACING

TEXT COIOUR

CHARACTER HEIGHT

CHARACTER ORIDNTATION

TEXT PATH

TEXT AIIGNDINT

CIAPACTER SET INDEX

AITERNATE CHARACTER SET INDEX

EIII BUNDLE INDEX

INTERTOR STYLE

EIII COLOUR

HATCH INDEX

PATTERN INDEX

PATTERN SIZE
GRS Metafile Interface

Item Notes
POLYIINE INDEX 21

LINE TYPE

IINE WIDTH SCAIE FACTOR 23

POLYIINE COLOUR INDEX 24

(1)

(2)

POLYMARKER INDEX 25

MARKER TYPE 26

MARKER SIZE SCAIE FACT 27

POLMMARKER COL INDEX 28

(1)

(2)

TEXT INDEX

TEXXT FONT AND PRECISION

TEXT FONT AND PRECISION 30

CHARACTER EXPANSION FACTOR 31

CHARACIER SPACING 32

TEXT COIOUR INDEX

CHARACTER HEIGTS

33

34

(4)

CHARACIER UR VECTOR

TEXT PATH

TEXT ALIGNIENT

TEXT FONT AND PRECISION

FIII AREA INDEX 37

EIIL AREA INTERIOR STYIE 38

EIII AREA COLOUR INDEX 40

FIII AREA STYLE INDEX 39

FIII AREA STYIE INDEX 39

RATTERN SIZE 
NOTES:

1) The default specification modes 'scaled' applies.

2) The default colour selection mode "indexed" applies.

3) Four CGM elements supply the relevant parameter values of the GKS TEXT FONT AND PRECISION item (either explicitly or implicitly by default): TEXT FONT INDEX, TEXT PRECISION, CHARACTER SET INDEX and ALTERNATE CHARACTER SET INDEX. The corresponding GKS font number may be detcrmined as described in sub-clause G.4.8.2. The occurrence of only one of the four CGM elcments uniquely indicates the mapping to GKS TEXT FONT AND PRECISION. The occurrence of more than one CGM element within one sequence in any order causes the corresponding GKS item to be relumed once.

4) Two CGM elements supply the relevant parameter values of the GKS CHARACTER VECTORS item (either explicitly or impliciuly by default): CHARACTER HEIGHT and CHARACTER ORIENTATION. The occurrence of only one of the two CGM elements uniquely indicates the mapping to GKS CHARACTER VECTORS. The occurrence of the two CGM elements within one sequence in any order causes the corresponding GKS item to be returned once.

5) TEXT FONT ASF and TEXT PRECISION ASF must be equal; they correspond to GKS TEXT FONT AND PRECISION ASF. HATCH and PATTERN INDEX ASF must be equal; they correspond to GKS FILL AREA STYLE INDEX ASF.

\section{G.6.8 Mapping of escape and external elements}

\section{CQY Element}

GKS Metafile Interface

Item Notes

$\begin{array}{llr}\text { ESCAPE } & \text { ESCAPE } & 6 \\ \text { MESSAGE } & \text { MESSAGE } & 5 \\ \text { APPICATION DATA } & \text { USER ITEM } & >100\end{array}$

NOTES

1) The 'action required' nag should be 'no_action'.

\section{G.6.9 Mapping of segment control elements}

cay slement

GKS Metafile Interface

Item Notes

COPY SEGENT

INTERITANCE FIITER

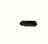

\section{G.6.10 Mapping of segment attribute elements}

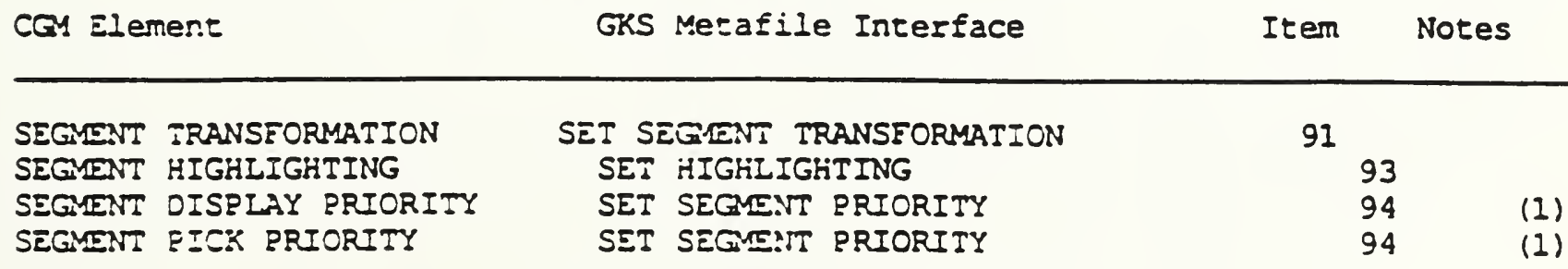


NOTES:

1)

Both CGM SEGMENT DISPLAY PRIORITY and SEGMENT PICK PRIORITY supply the parameter value of the GKS SET SEGMENT PRIORITY item. 


\title{
CGM Adden Jum 1
}

\author{
Part 2
}

\section{Draft Copy}

August 1989 
Add the following to the end of sub-clause 53:

3/8 for Segment Control Elements and Segment Auribuce Elements $3 / 13$ for Raster Elements

Page II

Add the following wo table 1:

\begin{tabular}{|c|c|c|c|c|}
\hline opoosde & Toit & & 8 bit & \\
\hline $\begin{array}{l}\text { BEGIN SEGMENT opeode } \\
\text { END SEGMENT opoode }\end{array}$ & $\begin{array}{l}3 / 0 \\
3 / 0\end{array}$ & $\begin{array}{l}2 / 5 \\
2 / 6\end{array}$ & $\begin{array}{l}03 / 0 \\
03 / 0\end{array}$ & $\begin{array}{l}02 / 5 \\
02 / 6\end{array}$ \\
\hline $\begin{array}{l}\text { NAME PRECISION opeode } \\
\text { MAXIMUM VDC EXTENT opcode } \\
\text { SEGMENT PRIORITY EXTENT opcode }\end{array}$ & $\begin{array}{l}3 / 1 \\
3 / 1 \\
3 / 1\end{array}$ & $\begin{array}{l}3 / 0 \\
3 n \\
3 / 2\end{array}$ & $\begin{array}{l}03 / 1 \\
03 / 1 \\
03 / 1\end{array}$ & $\begin{array}{l}03 / 0 \\
03 / 1 \\
03 / 2\end{array}$ \\
\hline 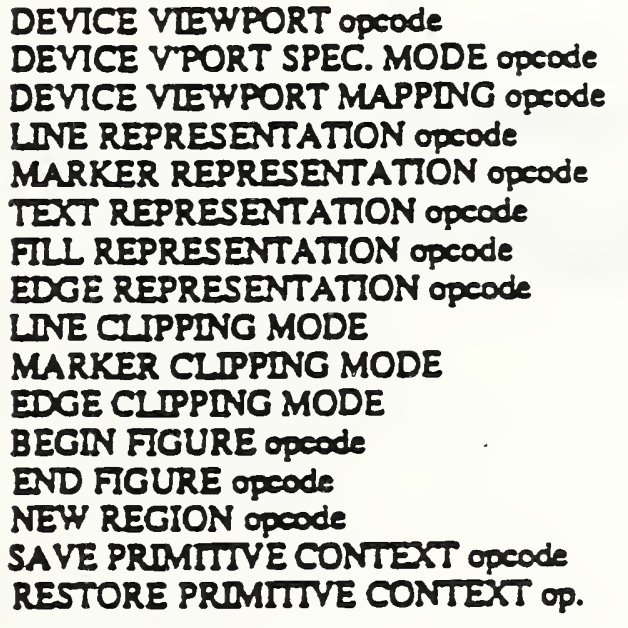 & $\begin{array}{l}3 / 2 \\
3 / 2 \\
3 / 2 \\
3 / 2 \\
3 / 2 \\
3 / 2 \\
3 / 2 \\
3 / 2 \\
3 \beta \\
3 \beta \\
3 \beta \\
3 \beta \\
3 \beta \\
3 \beta \\
3 \beta \\
3 \beta\end{array}$ & $\begin{array}{l}2 / 7 \\
2 / 8 \\
29 \\
2 / 10 \\
2 / 11 \\
2 / 12 \\
2 / 13 \\
2 / 14 \\
2 / 6 \\
2 / 7 \\
2 / 8 \\
29 \\
2 / 10 \\
2 / 11 \\
2 / 12 \\
2 / 13\end{array}$ & $\begin{array}{l}03 / 2 \\
03 / 2 \\
03 / 2 \\
03 / 2 \\
03 / 2 \\
03 / 2 \\
03 / 2 \\
03 / 2 \\
03 / \\
03 / 3 \\
03 / 3 \\
03 / \\
03 \beta \\
03 / 3 \\
03 \beta \\
03 \beta\end{array}$ & $\begin{array}{l}02 / 7 \\
02 / 8 \\
02 / 9 \\
02 / 10 \\
02 / 11 \\
02 / 12 \\
02 / 13 \\
02 / 14 \\
02 / 6 \\
02 / 7 \\
02 / 8 \\
02 / 9 \\
02110 \\
02 / 11 \\
02 / 12 \\
02 / 13\end{array}$ \\
\hline 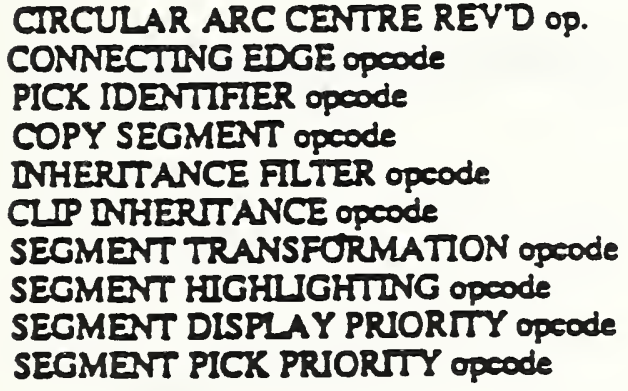 & $\begin{array}{l}3 / 4 \\
3 / 4 \\
3 / 6 \\
3 / 8 \\
3 / 8 \\
3 / 8 \\
3 / 8 \\
3 / 8 \\
3 / 8 \\
3 / 8\end{array}$ & $\begin{array}{l}2 / 8 \\
29 \\
3 / 2 \\
20 \\
2 \Omega \\
2 \Omega \\
2 / 3 \\
2 / 4 \\
2 / 5 \\
2 / 6\end{array}$ & $\begin{array}{l}03 / 4 \\
03 / 4 \\
03 / 6 \\
03 / 8 \\
03 / 8 \\
03 / 8 \\
03 / 8 \\
03 / 8 \\
03 / 8 \\
03 / 8\end{array}$ & $\begin{array}{l}02 / 8 \\
02 / 9 \\
03 / 2 \\
02 / 0 \\
02 / 1 \\
02 / 2 \\
02 / 3 \\
02 / 4 \\
02 / 5 \\
02 / 6\end{array}$ \\
\hline
\end{tabular}

Poge 27

Add the following sub-cinse afies sub-clause 6.12 .

\subsection{Viewport Point parameters}

A viewpon Poin (VP) is a pair of VC seales (Viewpon Coordinale) represencing the $x$ and $y$ coordinates of a poins in viewport specification space. A VC sealas is either an integer or real number aceording wo whecher VIEWPORT SPECIFICATION MODE is 'tracion of display surface.' 'millimeres with scale fxetot' or 'physical device units'.

When VIEWPORT SPECIFICATION MODE is 'fraction of display surface'. the encoding of the viewport point data rype is as described in 6.4 Coding Real Numbers. The size of the viewpor point parameters is limiled by the current REAL PRECISION value. 
When VIEWPORT SPCIFICATION MODE is 'millinetres with seale fector' of 'physical device unis', the encoding of the viewporr poins dars rype is as described in 6.3 Coding Integers. The size of the viewpor point paramelers is limiled by the current DNTEGER PRECISION value.

\subsection{Name parameters}

Name paranesers are coded as integers (Basic format) a NAME PRECISION.

Page 31

The following form sub-clauses 8.1.6 and 8.1.7

\subsubsection{BEGIN SEGMENT}

<BECIN.SEGMENT-opcode: 3/02/S>

oname: segment.identries

\subsubsection{END SEGME.T}

<END.SECMENT-opeode: 3/0 2/6>

Poge 34

Add the following to the cenumerued: element sed of sub-ciuse 8.2.11:

$$
\begin{array}{ll}
\text { Kinleger:2> } & \text { (VER.2 STATIC SET) } \\
\text { kinlegE:3> } & \text { (EXTENDED PRDMTIVES SET) } \\
\text { kinleger:4> } & \text { (VER.2 STATIC GKSM SET) }
\end{array}
$$

Page 36

The following form sub-clauses 8.2.16 108.218

\subsubsection{NA.ME PRECISION}

CNAME PRECISTON-opcode: $3 / 1 / 0>$

cinieger. largest-name-code + is

NOTE: The largest-name-code indicates how many bits ocerr in the largest possible magrinde for a name.

\subsubsection{MAXIMUM VDC EXTENT}

CMAXIMUM VDC EXTENT-Opcode: $3 / 1$ 3/1>

Poirtifist comers

spointisecond comes

\subsubsection{SEG.MENT PRIORITY EXTENT}

<SEGMENT PRIORTTY EXTENT Op-code: 3/1 3/2

cinteger. minimum-segment-priority-values

<integer.maximum-segment-priority-values

Page 38

The following form sub-clauses 8.3 .8 w 8.3 .15

\subsubsection{DEVICE. VIEWPORT}

<DEVICE-VIEWPORT-opoode: $3 / 22 /\rangle$

Cip: (urst comes )

sp : second comers )

\subsubsection{DFYICE VIEWPORT SPECIFICATION MODE}


CDEVICE VIEWPORT SPECIFICATION MODE op-code: 3/2 2/8>

<emumerued.VC specifies

cren: meoric seale factors

cenumerared VC speciries

$$
\begin{aligned}
& =\text { einteger:0s } \\
& 1 \text { integer.1> } \\
& \text { cintegr:2> }
\end{aligned}
$$

(fraction of drawing suriace)

(min with seale fector)

(physical device urits)

\subsubsection{DEVICE VIEWPORT MAPPING}

<DEVICE VIEWPORT MAPPING op-code: $3 / 2298$

Cenumeraced: isotropy-flag>

<emumerted horizonul-alignment-nag>

<enumerated verical alignment-flag>

<enumerzied isocropy-flag>

\begin{tabular}{|c|c|}
\hline 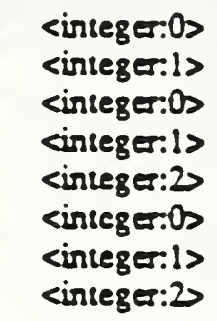 & 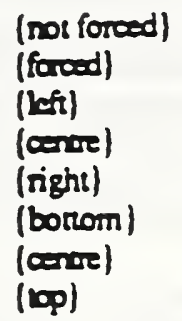 \\
\hline
\end{tabular}

<enumerated: horizonul-aligrment-nag>

<enumeraled:verieal-alignment-nag>

\subsubsection{LINE REPRESENTATION}

<LNE REPRESENTATION-opcode: 3/2 2/10>

<index: line.bundle.index

cindex: line-types

<line-width-specifies

<eolour-specities

Cindex: line-bundle-index

<index: line-types

$=$ Ppositive integer

= <integer. ls (solid]

1 cinseger. 20 (dast)

cirseger. 3> (dx)

cinieger: 4s (darh-da)

cinteger: S> (dxshdot-dox)

cinteger: negatives (privuse lixe type)

<line.width-spocifies

coolour-speciniess

cirreger: colour.inder
= Crel: line width-scale-factors (if LDNE WIDTH SPECIFICATION MODE is scaled)

1 SVDC: line wideh (if LINE WIDTH SPECIFIC MODE IS absolute)

- cinteger. colour indes (if COLOUR SELECTION MODE is indexed) ४RGB> (if COLOUR SELECTION MODE IS direa)

= oron-negaive integers

\subsubsection{MARKER REPRESENTATION}

CMARKER.REPRESENTATION-opcode: 3/2 2/11>

index: matke-bundle-inder

cindex: murker-iyper

Cnerke-size-specifies

<colour-specinties

<index: merker-bundle-index

<index: maker.typer

$=$ positive integas

$=$ <inieger. 1> cinseger: $2>$ cinteger: 3> <integer: 4> <inceger: 5>

$(d x)$

(plus)

(sterisk)

(circle)

(coos) 
entre-size-specifies

ceolour-speciñer

einteger. colour.indes

\subsubsection{TEXT REPRESENTATION}

STEXT-REPRESENTATION-opoode: $3 / 2$ 2/12

cindex: rexs-bundle-index

<integer. text-font-index>

cenumerued: texi-precisions

Gel: chrreter-specing

crel: expension-factors

<colour-specifles

cindex: iext-bundle-index>

cinteger. text-font-index>

cenumeried: text-precision

Ged: expersion-factors

\subsubsection{FILL REPRESENTATION}

CFIL-REPRESENTATION-opeode: 3/2 2/13>

eindex: III-bundle-inder

<enumeried interior-styles

ceolour speciriass

Sindex: hrich-indero

cindex: partern-inder>

<index:III-bundle-index>

cenumeraed: inserior styles

<eolour specifiess

<index: hurch-inder

<index: parem-index >
- Tenl: merker size-seale-factor

(if MARKER SDEE SPEC MODE is sealed)

1 SVDC: marke size

(if MARKER-SDCE SPEC MOC : is absolute)

= cinteger. colour inder> (if COLOUR SELECTION MODE IS indexed]

1 QRGB>

(if COLOUR SELEC MODE is direet)

= cron-neguive inlegers
- Tositive integers

= Sositive integers

= einteger.0s (string)

1 einteger.ls (charaer)

1 einteger.2s (stroke)

= cron-negaive reals
- Tositive integes

- cinteger.0s lhollow

sinteger.ls (solid cinteger.2) (pamen

cinieger.3) (thatch

cinteger.4s lempry

einugernegatives (privale style)

Cinieger:colour inders

(if COLOUR SELECIION MODE is indexed) CRGB>

(if COLOUR SELECTION MODE is direet)

- Qinteger.ls

dinteger.2s

inieger.3>

cinieger.4s

integerss

inieger.6>

cintegernegaives

(borioned]

(verieal)

(positive slope)

[neguive slope)

(horiztveried cross)

(positivehreg ctoss)

(privare styles)

\subsubsection{EDGE REPRESENTATION}

<EDGE-REPRESENTATION-opcode: 3/2 2/14>

<inclex: edge-bunde-inder

$=$ Positive integers 


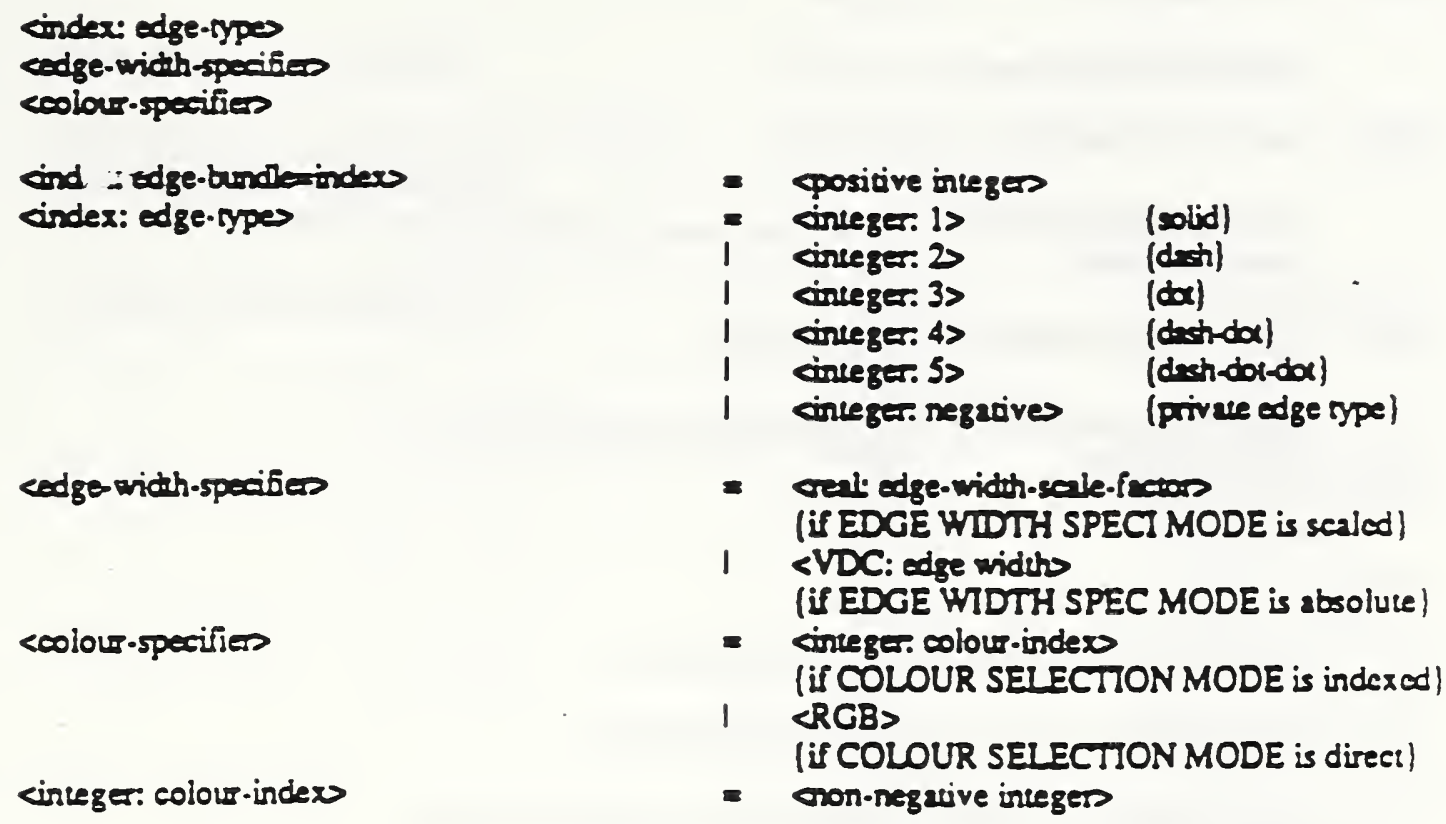

cinteger. negacives (privule edge rype)

- creali dige-width-sole-fectos (if EDGE WIDTH SPECI MODE is sealed)

I SVDC: edge widith (if EDGE WIDTH SPEC MODE is absolute)

- cinteger. colour-index (if COLOUR SELECTION MODE is indexed)

1 <RGB> (if COLOUR SELECTION MODE is diree:)

- Cron-neguive iniegers

(solid)

(dast)

(do)

(dastodo)

(dish-dot-dox)

Page 40

The following form sub-ciauses 8.4 .7 w $8.4 .1 \mathrm{~s}$

\subsubsection{LNE CLIPPING MODE}

LINE CLIPPDNG MODE-opeodes: 3/3 2/6> <exumerued elipping modes

<enumerued elipping modes

\section{8. $\$ .8$ MARKER CLIPPING MODE}

$$
\begin{array}{ll}
\text { integer.0s } & \text { (bous) } \\
\text { integer.1s } & \text { (shepe) } \\
\text { sinteger.2) } & \text { (locus then shape) }
\end{array}
$$

LLNE CLIPPDG MODE-opcodes: $3 / 3$ 2/7

cenumerated elipping modes

cenumeraed elipping modes

$$
\begin{array}{ll}
\text { sinteger:0s } & \text { (bous) } \\
\text { sinteger.1s } & \text { (shipe) } \\
\text { integer.2) } & \text { (locus then shape) }
\end{array}
$$

\subsubsection{EDGE CLIPPING MODE}

LLNE CLIPPING MODE-opeodes: $3 / 3$ 2/8>

cenrmented elipping modes

cerwmeried elipping modes

$\begin{array}{ll}\text { inleger.0s } & \text { (bous) } \\ \text { integer.1s } & \text { (shipe) } \\ \text { inieger.2) } & \text { (bous then shape) }\end{array}$

\subsubsection{BEGIN FGURE}

¿BEGDN FGURE-opoode 3/3 2/9

\subsubsection{END FIGURE}

LEND FIGURE-opoode: $3 \beta$ 2nO 


\subsubsection{NEW REGION}

LNEW REGION-opeode: $3 / 3$ 2/11>

\subsubsection{SAVE PRIMITIVE CONTEXT}

SAVE PRIMITIVE ATTRIBUTES-opcode: $3 / 32 / 2>$

enime contexo

\subsubsection{RESTORE PRIMITIVE CONTEXT}

<RESTORE PRDMTTIVE ATTRBBUTES-opcode: $3 / 3$ 2/13> conme contero

Page 45

The following form sub-clause 8.5 .20 and 8.5 .21

\subsubsection{CIRCULAR ARC CENTRE REVERSED}

CCIRCULAR ARC CENTRE REVERSED-opcode: $3 / 4$ 2/8>

Soint centrepoino

SVDC: DX_suro

SVDC: DY_surd

SVDC: DX_end

SVDC: DY_end

SVDC: radius

\subsubsection{CONNECTING EDGE}

<CONNECTING EDGE-opcode: 3/4 2/9

Pages4

The following forms sub-cisuse 8.6 .36

\subsection{PICK IDENTIFIER}

SPICK-ID-opeode: 3/6 3/2

corme: pick-identiners

Page 56

The following forms sub-diuse $\$ .9$

\subsubsection{COPY SEGMENT}

COPY SEGMENT-opeode: 3/8 20S

conne segment-identifies

erreformation mavio

cenmeractsegners transformation applieation

erestormation matrix

- Geal: all > Creal: al2> creal: $21>$ Geal: $222>$ Svde: : $213>$ sude : 223 >

cenmentedsegment transformation application

- <integet:0>

$(n)$ 


\subsubsection{INHERTTANCE FLTER}

LWHERTTANCE-FILTER-Opcode: $3 / 8$ 2/1>

cenumerated: filu-relection-liso-

<enumer rtedt selection seaing>

cenumerued: filuer-selection-lisD

(line burdle index)

(line type)

(line widah)

(line colour)

(line clipping mode)

(marke bundie index)

(maker type)

(marker size)

(marker colour)

(marker clipping mode)

(lext bundle index)

(text font index)

(exe procision]

(character expension factor)

[churecter spacing]

(text colour)

(character height]

(character orientation)

(Lest pach)

(Lext alignment)

(Fill bundle index)

(interior soyle)

(ill colour)

(hatch index)

(paneen index)

(edge bundle index)

(edge type)

(edge width)

(edge colour)

[edge visibility)

(adge clipping mode)

(iil reference point)

(pareon size)

(auxiliary colour)

(ransperency)

(line survibutes)

(marker aurubure)

(text atribures)

(charncter sursibutes)

(fill arsibures)

(edge unibures)

(pautem auributes)

(ouppris control)

(picts identifies)

(all auribues and control)

(iii)

(line type ASF)

(line wideh ASF)

(line colour ASF)

(marker rype ASF)

[matker size ASF]

[maker colour ASF]

[text font index ASF]

[Lxi PRECISION ASF\}

(character expansion factor 
<enumerated: selection setling>

$$
\begin{aligned}
& \text { cinegers } \\
& \text { ciniegers } \\
& \text { ciniegers } \\
& \text { cintegers } \\
& \text { ciniegers } \\
& \text { cintegers } \\
& \text { cintegers } \\
& \text { cintegers } \\
& \text { cintegers } \\
& \text { integer.46> } \\
& \text { cinteger.47> } \\
& \text { cinteger.48> } \\
& \text { cinteger.49> } \\
& \text { cinteger.50> } \\
& \text { cinteger.51> } \\
& \text { cinteger.52> }
\end{aligned}
$$

$=\quad$ sintege:0s

1 cinieger:1> (chrracter specing ASF)

(text colour ASF)

(interior style ASF)

(fill colou ASF]

(harch index ASF)

(parren index ASF]

(edge type ASF)

(edge widis ASF]

(edge colour ASF)

(line ASFs)

(marite- ASFs)

(Lext ASFs)

(chrencter ASFs)

(III ASFs)

(edge ASFs]

(dil ASFs)

(state_list]

(sezmens)

\subsubsection{CLIP INHERITA.ICE}

<CLIP INHERTTANCE-opcode: $3 / 8$ 2/2 enumerued: slip-inheritances <enumerued: secting>

= <integer:0s

1 cinteger:1> (state_liso (intersection)

\subsubsection{SEG.ME.IT TRANSFOR.MATION}

<SEGMENT-TRANSFORMATION-opcode: $3 / 82 / 3$ > Orame: segment-identifies <ransformation marrix>

¿trassformation matrix>

= Ceal: all > Geal: $212>$ Crai: $221>$ Geal: $222>$ Svde : $213>$ sude : $223>$

\subsubsection{SEG.MENT HIGHLIGHTING}

CSEGMENT.HIGHLIGHTING-opeode: 3/8 2/4>

crame: segment-identifies

cenumerued: segment-highlighting>

cramesegment identiriers

cenumerued: segment-highlighting>

- cinteger

- cinteger. 0s

1 cineger. 1s

(romsi)

(highlighred)

\subsubsection{SEGMENT DISPLAY PRIORITY}

ESEGMENT.PRJORTYY-opeode: 3/8 2/S>

Crme: segment-idantifies

<integer: segment-display-prioritys

<inieger. segment-display-priority>

= Spositive integers

\subsubsection{SEG.HE.IT PICK PRIORITY}

-

<SEGMENT-PICK.PRIORITY-opcnde: 3/8 2/6>

orame: segment-identifies 
cinteger: pick-prioritys

cinteger: pick priority>

- positive inlegers

Page 56

Add the following to the and of clause 9

NAME PRECISION $\quad: \quad 10$

Page 60

Add the following to the end of Anser A

come precision values

sviewpor poino

crames

1 cenlocals

<x2 matrix of reals>

$=$ ciriegers

Qxl matrix of vdess

$\begin{array}{ll}=\quad \text { seal (4) } & (\sec 8.9) \\ =\quad \text { sode valuer (2) } & {[\sec 8.9\}}\end{array}$



CGM Addendum 1

Part 3

Draft Copy

August 1989 
Page 16

Add the following to table 1:

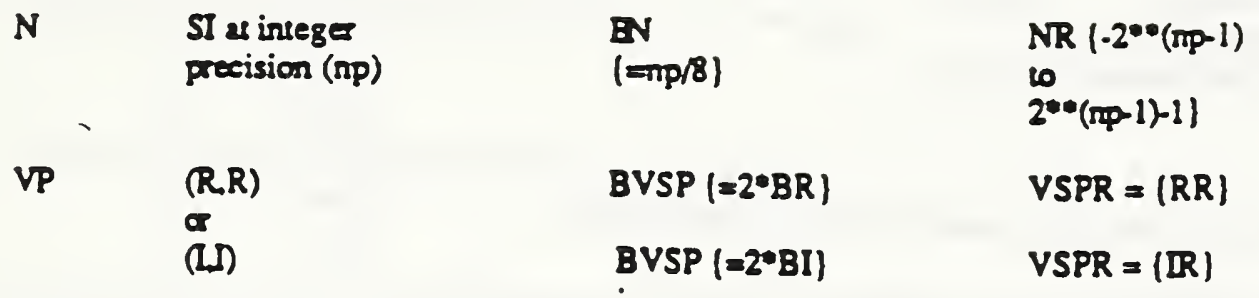

Page 19

Add the following to Table 2 .

8 Segment Control and Segment Atribute elements

Page 20

Add the following wo table 3:

$\begin{array}{llllll}\text { BEGN SEGMENT } & 6 & N & B N & N R & \mathrm{~N} / \mathrm{a} \\ \text { END SEGMENT } & 7 & \mathrm{n} / \mathrm{a} & 0 & \mathrm{~N} / \mathrm{a} & \mathrm{N} / \mathrm{a}\end{array}$

Code Descripion

6 BECDN SEGMENT: has 1 parmeter.

P1: (segment name) segment idendifer

7 END SEGMENT: has no paramesers

Page 21

Add the following wo table 4:

$\begin{array}{llllll}\text { NAME PRECISION } & 16 & N & \text { BN } & 8.16 .24 .32 & 16 \\ \text { MAXIMUM VDC EXTENT } & 17 & 2 P & \text { 2BP } & \text { VDCR } & \text { VDC EXTENT } \\ \text { SEC PRIORTY EXTIENT } & 18 & 21 & \text { 2BI } & \text { IR } & 0.255\end{array}$

Code Descripion

16 NAME PRECISION: has I purumeter.

P1: (inseger) name precision: 8.26.24 or 32 are the only valid values

17 MAXIMUM VDC EXTENT: has 2 pormerers:

P1: (point) litrs poins

P2: (poins) second poins

18 SEGMENT PRIORTIY EXTENT: has 2 parmeters.

P1: (integer) miximum segnent priority value

P2: (intege) maximum segmess priority value 
Page 22

Add wo the note P2 of METAFILE ELEMENT LIST:

ver-stric

extended-primivives

ver2-gkem-sratic $(-1,4)$

Page 24

Add to the end of the noce P2 for SCALDG MODE:

Note thas this parameter is always encoded as Flosing Point regardless of the value of the fixed/floating flag of REAL PRECISION.

Page 24

Add the following to table S:

\begin{tabular}{|c|c|c|c|c|c|}
\hline $\begin{array}{l}\text { DEVICE VIEWPORT } \\
\text { DEVICE VIEWPORT }\end{array}$ & $\begin{array}{l}8 \\
9\end{array}$ & $\begin{array}{l}\text { 2VP } \\
\text { E.R }\end{array}$ & $\begin{array}{l}2 B V P \\
B E+B R\end{array}$ & $\begin{array}{l}\text { VPR } \\
(0,1.2\}\end{array}$ & $\begin{array}{l}\text { see below } \\
0 . \text {. }\end{array}$ \\
\hline MAPPDNG & 10 & $3 E$ & 3BE & $\begin{array}{l}(0.1) \\
(0.1 .2\} \\
(0,1.2)\end{array}$ & $\begin{array}{l}1 \\
0 \\
0\end{array}$ \\
\hline LNE REPRESENTATION & 11 & $\begin{array}{l}2 D X . \\
\text { VDC or } \\
\text { R),CO }\end{array}$ & $\begin{array}{l}2 B \Gamma X+ \\
\text { (BVDC or } \\
\text { BR) }+ \text { BCO }\end{array}$ & $\begin{array}{l}+ \text { LXR.DXR. } \\
+ \text { +VDCR or } \\
+ \text { +RR.COR }\end{array}$ & $\mathbf{n} / \mathbf{a}$ \\
\hline MARKER REPTION & 12 & $\begin{array}{l}\text { 2DX. } \\
\text { (VDC or } \\
\text { R),CO }\end{array}$ & $\begin{array}{l}2 B D X+ \\
(B V D C \text { or } \\
B R)+B C O\end{array}$ & $\begin{array}{l}+ \text { +DR.DXR, } \\
+ \text { +VDCR or } \\
+ \text { +RR,COR }\end{array}$ & $\mathbf{n} / \mathbf{a}$ \\
\hline TEXT REPRESENTATION & 13 & $\begin{array}{l}2 D X \\
E \\
2 R . C O\end{array}$ & $\begin{array}{l}2 B X_{+} \\
B E+ \\
2 B R+B C O\end{array}$ & $\begin{array}{l}+ \text { DXR. } \\
(0,1,2) \\
\text { RR,COR }\end{array}$ & $\mathbf{n} / \mathbf{a}$ \\
\hline FILL REPRESENTATION & 14 & $\begin{array}{l}\text { DX. } \\
\text { E.CO. } \\
2 D X\end{array}$ & $\begin{array}{l}B D X+ \\
B E+B C O+ \\
28 D X\end{array}$ & $\begin{array}{l}+D R, \\
(0.4), C O R . \\
D K R_{0}+D \times R\end{array}$ & a/a \\
\hline EDGE REPRESENTATION & 15 & $\begin{array}{l}\text { 2DX, } \\
\text { NDC or } \\
\text { R).CO }\end{array}$ & $\begin{array}{l}28 D X+ \\
\text { (BVDC or } \\
\text { BR)+BCO }\end{array}$ & $\begin{array}{l}+ \text { +DR.DXR. } \\
++ \text { VDCR or } \\
++R R . C O R\end{array}$ & $\mathbf{N} / \mathbf{2}$ \\
\hline
\end{tabular}

Code Decaripion

8 DEVICE VIEWPORT: has 2 parmeters:

Pl: (vp) furst point

P2: (vp) second poins

The defauls DEVICE VIEWPORT is the antire device view surface if the latter is rectungular. or the largest rectroguier subset having the desired aspees ravia. if the view suriee is not rectmgula. The default is sel so thes the 'first point' is beiow and to the left of the 'second point' $a$ seen by the viewer.

DEVICE VIEHPORT SPECIFICATION MODE: he 2 prameter:

P1: (enumented) viewpor specibeation uriks: valid values are

0
1 millimetres with seale fector
2 physical device units

P2. (rea) metric seale fector, ignored if $\mathrm{Pl}=0$ or $\mathrm{Pl}=2$

- Note thas this paramear is always encoded as Flouing Point regardless of the value of the fixed/noating flag of REAL PRECISION. 
P1: (enumerated) isotropy flag: valid values are:

0 nor forced

1 hored

P2. (enumerated) horizontal sligrment flag: valid values are:

0 lefis

1 contre

2 righs

P3: (enumernted) verical aligmens flag: valid values are.

0 botrom

1 conere

$2 \operatorname{cop}$

11 LINE REPRESENTATION: has 4 parameter:

P1: (index) line bundle index

P2. (index) line type: the following values are sundardized:

1 solid

2 dash

3 dax

4 dashido

5 dach-dodot

regative for private use

P3: (vole or real) absolute line widh or line width seale factor

P4: (colour) line colour. its form depends on COLOUR SELECTION MODE.

12 MARKER REPRESENTATION: has 4 parameters:

Pl: (index) marke burdle index

P2: (index) marker type: the following values are sundardized:

$\begin{array}{ll}1 & \text { dox } \\ 2 & \text { plus } \\ 3 & \text { ascerisk } \\ 4 & \text { cincte } \\ 5 & \text { coos }\end{array}$

regative for privare use

P3: (vode or real) absolure marker width or marker size seale factor

P4: (colour) marke colour. its form depends on COLOUR SELECTION MODE.

13 TEXT REPRESENTATION: has 6 parmerers:

P1: (index) text bundle index

P2: (index) text fons index

P3: (entrnerued) text precision: valid values \&re:

0 sosing

1 chureser

2 croke

P4: (real) churecter spacing

PS: (real) cherecer expension ferer

P6: (colour) lext colour, its form depends on COLOUR SELECTION MODE

14 FIL REPRESENTATION: has 5 parameters:

P1: (index) In urea bundle index

P2. (enumeruted) interior style: valid values are:

0 hollow

1 solid

2 paren

3 ham

4 empry

- P3: (colour) fill colour: its form depends on COLOUR SELECTION MODE

P4: (index) hasch index: the following values are suandardized:

1 horizonial 


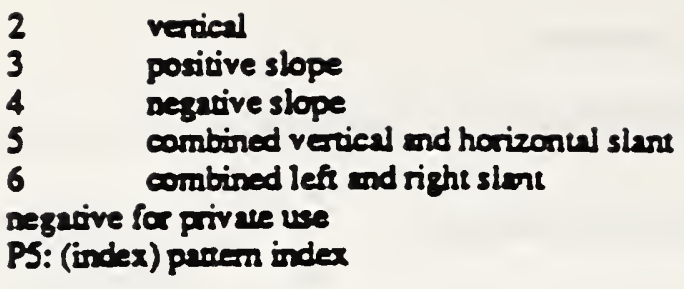

15 EDGE REPRESENTATION: has 4 peramelers:

P1: (index) edge bundle index

P2: (index) edge type the following values are stendertized.

1 solid

2 deh

3 dex

4 dxhodo

5 dach-dodot

negarive for private use

P3: (vde or real) absolute edge width or line width scale fachor

P4: (colour) edge colour. its form depends on COLOUR SELECTION MODE.

Page 26

Add the following to table 6:

$\begin{array}{llllll}\text { LNE CLIPPDNG MODE } & 7 & \text { E } & \text { EE } & 10.1 .21 & 0 \\ \text { MARKER CLIPPING MODE } & 8 & \text { E } & \text { EE } & 10.1 .21 & 0 \\ \text { EDGE CLIPPLNG MODE } & 9 & \text { E } & \text { EE } & 10.1 .21 & 0 \\ \text { BECD FIGURE } & 10 & \mathrm{n} / \mathrm{a} & 0 & \mathrm{n} / \mathrm{a} & \mathrm{n} / \mathrm{a} \\ \text { END FIGURE } & 11 & \mathrm{n} / \mathrm{a} & 0 & \mathrm{n} / \mathrm{a} & \mathrm{n} / \mathrm{a} \\ \text { NEW REGION } & 12 & \mathrm{n} / \mathrm{a} & 0 & \mathrm{n} / \mathrm{a} & \mathrm{n} / \mathrm{a} \\ \text { SAVE PRIMIITE CONTEXT } & 13 & \mathrm{~N} & \mathrm{BN} & \mathrm{NR} & \mathrm{n} / \mathrm{a} \\ \text { RESTORE PRIMITIVE CONTEXT } & 14 & \mathrm{~N} & \mathrm{BN} & \mathrm{NR}\end{array}$

7 LINE CLIPPDNG MODE: hes 1 purneter.

P1: (enumerated) clipping mode: valid values are:

0 beers

1 shipe

2 beus then shape

8 MARKER CLIPPDNG MODE: has 1 puraneter.

P1: (enumerued) clipping mode: valid values are:

0
1 bews
2 beis then shype

9 EDGE CLIPPDG MODE: hs I parmeler.

P1: (enumerued) elipping modes valid values are

0 bers

1 the

2 bens then shape

10 BECWN FIGURE: hes no parnetess

11 END FIGURE: he no parmeters

12 NEW REGION: hes no permevers

13 SAVE PROMITIVE CONTEXT: has 1 pernneler.

Pl: (name) context name 
P1: (nume) context name

Page 28

Add the following to table 7:

CIRCULAR ARC CENTRE

\begin{tabular}{|c|c|c|c|c|}
\hline $\begin{array}{l}\text { REVERSED } \\
\text { CONNECTING EDGE }\end{array}$ & 20 & $\begin{array}{l}\text { P.4VDC. } \\
\text { VDC } \\
\mathrm{n} / \mathrm{a}\end{array}$ & $\begin{array}{l}B P+4 B V D C+ \\
B V D C \\
0\end{array}$ & $\begin{array}{l}\text { VDCR. VDCR. } \\
+ \text { VDCR } \\
\text { Rta }\end{array}$ \\
\hline
\end{tabular}

Code Description

20 CIRCULAR ARC CENTRE REVERSED: has 6 parancters:

P1: (point) centre of circle

P2: (vdc) delu $X$ for sturt vector

P3: (vde) delu $Y$ for sturt vector

P4: (vde) delu $X$ for end vector

PS: (vde) delta $Y$ for end vecer

P6: (vdc) radius of circle

21. CONNECIDNG EDGE has no parameters

Page 33

Add the following to table 8:

$\begin{array}{lllllll}\text { PICK IDENTIFIER } & 36 & \mathrm{~N} & \mathrm{BN} & \mathrm{NR} & 0\end{array}$

Code Descripion

36 PICK IDENTIFIER: has 1 parameter:

P1: (name) pick identifies

Page 39

The following forms sub-clause 7.10

\subsection{Segment Control and Segment Attribute Elements}

Table 11 - Excoding of segment control and segment atribute elements

\begin{tabular}{|c|c|c|c|c|c|}
\hline $\begin{array}{l}\text { Elemens } \\
\text { Class } 8\end{array}$ & घ & $\begin{array}{l}\text { Param } \\
\text { Type }\end{array}$ & $\begin{array}{l}\text { Purameler } \\
\text { List } \\
\text { Lenght }\end{array}$ & $\begin{array}{l}\text { Parameler } \\
\text { Rarge }\end{array}$ & Defaule \\
\hline COPY SEGMENT & 1 & $\begin{array}{l}\text { N.4R. } \\
\text { 2VDC. } \\
E\end{array}$ & $\begin{array}{l}B N+4 B R+ \\
2 B V D C+ \\
\text { EE }\end{array}$ & $\begin{array}{l}\text { NR.RR. } \\
\text { VDCR. } \\
\text { (Q.1) }\end{array}$ & .0 \\
\hline $\begin{array}{l}\text { DNHERTTANCE FILTER } \\
\text { CLIP INHERTANCE } 3\end{array}$ & $\stackrel{2}{E}$ & & $\begin{array}{ll}(n+1) B E \\
(0.1\}^{0} 0\end{array}$ & $\{0.431 .\{0.1\}$ & .1 \\
\hline SEGMENT TRANSFORM & 4 & & $\begin{array}{l}\text { BN+4BR+ } \\
2 B V D C\end{array}$ & $\begin{array}{l}\text { NR.RR. } \\
\text { VDCR }\end{array}$ & $\begin{array}{l}\Omega / 2.1 .0 .0 .0 \\
1.0\end{array}$ \\
\hline $\begin{array}{l}\text { SEGMENT HIGHUGHTING } \\
\text { SEG DISPLAY PRIORITY } \\
\text { SEGMENT PICK PRIORITY }\end{array}$ & $\begin{array}{l}5 \\
6 \\
7\end{array}$ & $\begin{array}{l}\text { N.E } \\
\text { N.I } \\
\text { N.I }\end{array}$ & $\begin{array}{l}B N+B E \\
B N+B I \\
B N+B I\end{array}$ & $\begin{array}{l}\text { NR. }(0.1) \\
\text { NR.IR } \\
\text { NR.IR }\end{array}$ & $\begin{array}{l}\text { n/20 } \\
n / 2 \text { sec below } \\
n / 2 \text { sec below }\end{array}$ \\
\hline \multicolumn{6}{|l|}{ Code Descripion } \\
\hline COPY SEGMENT: has 8 & $m$ & & & & \\
\hline
\end{tabular}


The nexs 6 parmeters are components of a $3 \times 2$ marix of the form:

IP2 P3 P61

IP4 PS P7I

where

P2: (real) a scule component

P3: (rea) $x$ roution component

P4: (real) y roution component

PS: (red) y seale component

P6: (vde) $x$ transiacion companens

P7: (vde) y translation component

P8: (enumerued) segnent ensformation application: valid values are:

0: $\quad$ is

1: $y=$

2 DNHERITANCE FITER: has two parameters. The first is a list of up w designators. The socond is a single selting vaiue.

P1: (enumered list) list of one or more of:

0 line bundle index

1 line type

2 line widh

3 line colour

line clipping mode

4 marke bundle index

5 make type

6 marke size

7 marker colour

merker elipping mode

8 lexi bundle index

9 Lext fons index

10 cext precision

11 character expersion factor

12 chareere specing

13 text colour

14 chrreer heighs

15 chrreter orientacion

16 Lext puth

17 cext alignment

20 fill bundle index

21 interior styie

2 (1) colour

23 huch index

24 pawern index

5 odge bundle index

20 edge rype

21 dige with

28 decolour

2 edge visibility

edge clipping mode

30 min reference point

31 parem size

32 exiliery colour

33 resperexy

34 line atribules

35 miter unibures

36 vat ausibutes

37 chernerer awibutes

38 Gill atributes

39 edge atoribures

40 parem atribules

42 ouput control

pick identifia

43 all anribures and consol

21 


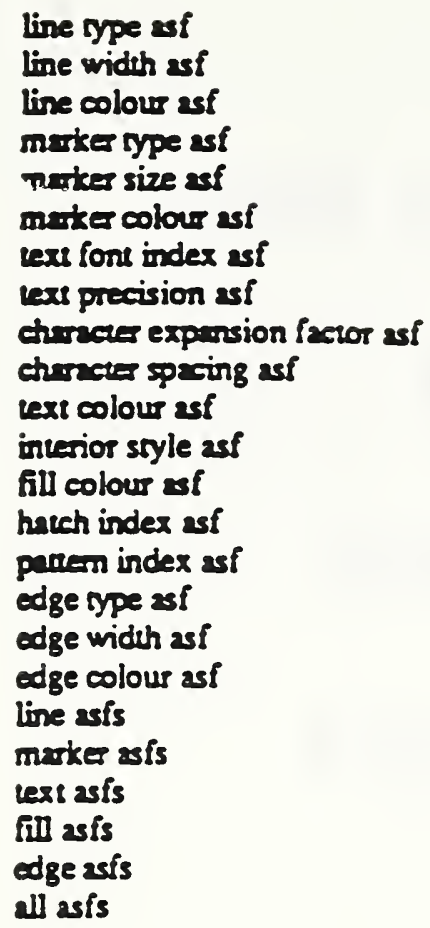

P2: (enumerted) seuling: valid values are:

0 strite_list

1 segonest

3 CUP INHERT TANCE: has I parameter

P1: (erumeruted) clip inheriunce: valid values are:

0 sute_liss

1 segmens

SECMENT TRANSFORMATION: has 2 parametcr:

P1: (neme) segment identifie

The second paranete consists of a $3 \times 2$ matrix of the form:

P2: (2x2 marix of reals. $2 \times 1$ mavix of vics)

SEGMENT HIGHLGHTING: has 2 parameleTs:

P1: (nщme) segmens identirie

P2: (enumerued) highlighting valid values are:

0 sormal

1 bighlighted

\section{SEGMENT DISPLAY PRIORTYY: has 2 paramerers:}

Pl: (arme) secment identifie

P2. (imege) sement diplay priority

The def mils of the segment display priority is equal to the minimum segmens priority value (see sub-clause 73)

SEGMENT PICK PRIORITY: has 2 parneters:

P1: (name) segmens identifier

P2: (inueger) segment pick priority

The defauls of the segment pick priority is equal wo the minimum segment priority value (see sub-clause 7.3) 
Add the following wo the end of clause 8:

NAME PRECISION

16

Page 12

Add the following to the list of references:

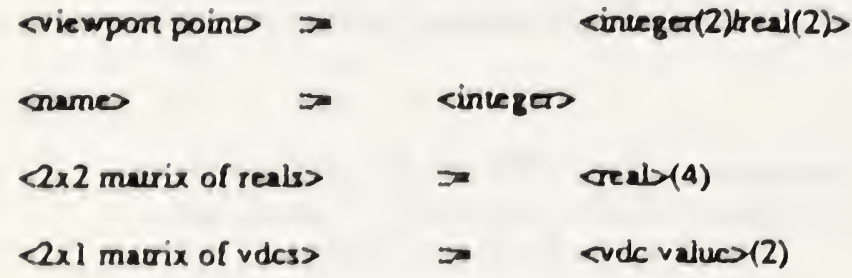

Page 48

Add the following wo the list of elements:

\begin{tabular}{|c|c|c|}
\hline Class & $\begin{array}{l}\text { Elcment } \\
\text { Codk }\end{array}$ & Elemeni Name \\
\hline 0 & 6 & BEGIN SEGMENT \\
\hline 0 & 7 & END SEGMENT \\
\hline 1 & 16 & NAME PRECISION \\
\hline 1 & 17 & MAXMMUM VDC EXTENT \\
\hline 1 & 18 & SEGMENT PRIORTTY EXTENT \\
\hline 2 & 8 & DEVICE VIEWPORT \\
\hline 2 & 9 & DEVICE VIEWPORT SPECIFICATION MODE \\
\hline 2 & 10 & DEVICE VIEWPORT MAPPING \\
\hline 2 & 11 & LINE REPRESENTATION \\
\hline 2 & 12 & MARKER REPRESENTATION \\
\hline 2 & $\mathbf{B}$ & TEXT REPRESENTATION \\
\hline 2 & 14 & FILL REPRESENTATION \\
\hline 2 & is & EDGE REPRESENTATION \\
\hline 3 & 7 & LINE CLIPPING MODE \\
\hline 3 & 8 & MARKER CLIPPING MODE \\
\hline 3 & 9 & EDGE CUPPING MODE \\
\hline 3 & 10 & BEGIN FIGURE \\
\hline 3 & 11 & END FIGURE \\
\hline 3 & 12 & NEW REGION \\
\hline 3 & 13 & SAVE PRIMITVE CONTEXT \\
\hline 3 & $\mathbf{M}$ & RESTORE PRIMITIVE CONTEXT \\
\hline 4 & $\mathbf{D}$ & CIRCULAR ARC CENTRE REVERSED \\
\hline 4 & $\boldsymbol{n}$ & CONNECTING EDGE \\
\hline s & 36 & PICK IDENTIFIER \\
\hline 8 & 1 & COPY SEGMENT \\
\hline 8 & 2 & DNHERITANCE FILTER \\
\hline 8 & 3 & CUIP INHERTTANCE \\
\hline 8 & 4 & SEGMENT TRANSFORMATION \\
\hline 8 & 5 & SEGMENT HIGHLIGHTING \\
\hline 8 & 6 & SEGMENT DISPLAY PRIORTTY \\
\hline 8 & & \\
\hline
\end{tabular}


CGM Addendum i

Part 4

Draft Copy

August 1989 

Add the following to the end of sub-cluuse 53.5

\begin{tabular}{|c|c|c|c|}
\hline $\mathbf{N}$ & : & لك & (n:me) \\
\hline ve & : & CR>Kl & $\begin{array}{l}\text { (viewport coordinrte den depending } \\
\text { on DEVICE VIEWPORT SPECIFICATION MODE) }\end{array}$ \\
\hline VPODNTREC & $::=$ & $<V C><S$ & $B<V C>$ \\
\hline VP & $=$ & $\begin{array}{l}\text { SVPODN } \\
\text { CRIGHT }\end{array}$ & 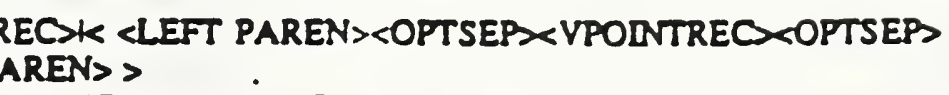 \\
\hline
\end{tabular}

(COORDDNATE in viewpor specification space. Pr n theses are optional. If they are used. they shall group exsedly two resl or inleger numbers, depending on UEVICE VIEWPORT SPECIFICATION MODE. The parenchesized form is intended to aid readability of the menrile]

\begin{tabular}{|c|c|c|}
\hline ROWMATRDX & $=$ & 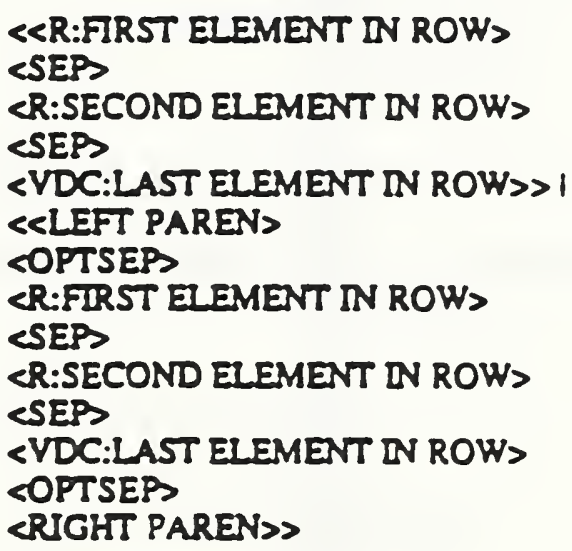 \\
\hline TM & $=$ & $\begin{array}{l}\text { CROWMLATRX> } \\
\text { eSEP> } \\
\text { SROWMATRX> }\end{array}$ \\
\hline
\end{tabular}

$12 \times 3$ transformation marrix in row-major order. Parentheses are opxional. If they are used, they shall group exacty two resi mumbers and ane VDC number. The perenthesized form is intended wo sid readability of the melarile. if there are not tree rumbers in each parenthesised group, the metarile is non-conforming interchange. Any urempred error reeovery or exception handling which a metafile interpreter may use in this situation is neither detised not constrined by ISO 8632/4. A mevafile interpreter nee not use the parentheses in parsing; in this case, they are trented as SPACE charecters sathe than as NULL chancters (i.en they act as sofi delimiters).)

Page 12

Add the following to the end of sub-cimse 5.43
All
COPY
EXTENDED
FICURE
FILTER
FORCED
FRACTION
GKSM
DIERSECTION
LOCUS
MATRX
NAME
NEW
PICK -
PRIMITIVES
REGION 
Page 12

Add the following wo the end of sub-clause 5.4.4

\begin{tabular}{|c|c|}
\hline $\begin{array}{l}\text { ATTRIBUTE(S) } \\
\text { CLPPING } \\
\text { CONNECIING } \\
\text { CONTEXT } \\
\text { DEVICE } \\
\text { DISPLAY } \\
\text { HLGHLIGHTING } \\
\text { IDENTIFIER } \\
\text { NHERTIANCE } \\
\text { MAPPING } \\
\text { MULIMETER } \\
\text { PHYSICAL } \\
\text { PIXE } \\
\text { PRIMIIIVE } \\
\text { PRIORITY } \\
\text { REPRESENTATION } \\
\text { RESTORE } \\
\text { REVERSED } \\
\text { SEGMENT } \\
\text { TRANSFORMATION } \\
\text { VIEWPORT }\end{array}$ & $\begin{array}{l}\text { ATTR } \\
\text { CLP } \\
\text { CONN } \\
\text { CONT } \\
\text { DEY } \\
\text { DISP } \\
\text { HTHUGHT } \\
\text { D } \\
\text { INH } \\
\text { MAP } \\
\text { MM } \\
\text { PHY } \\
\text { PEL } \\
\text { PRIM } \\
\text { PRI } \\
\text { REP } \\
\text { RES } \\
\text { REV } \\
\text { SEC } \\
\text { TRAN } \\
\text { VP }\end{array}$ \\
\hline
\end{tabular}

Page 14

Add the following wo the end of sub-ciause 5.4.5

\begin{tabular}{|c|}
\hline 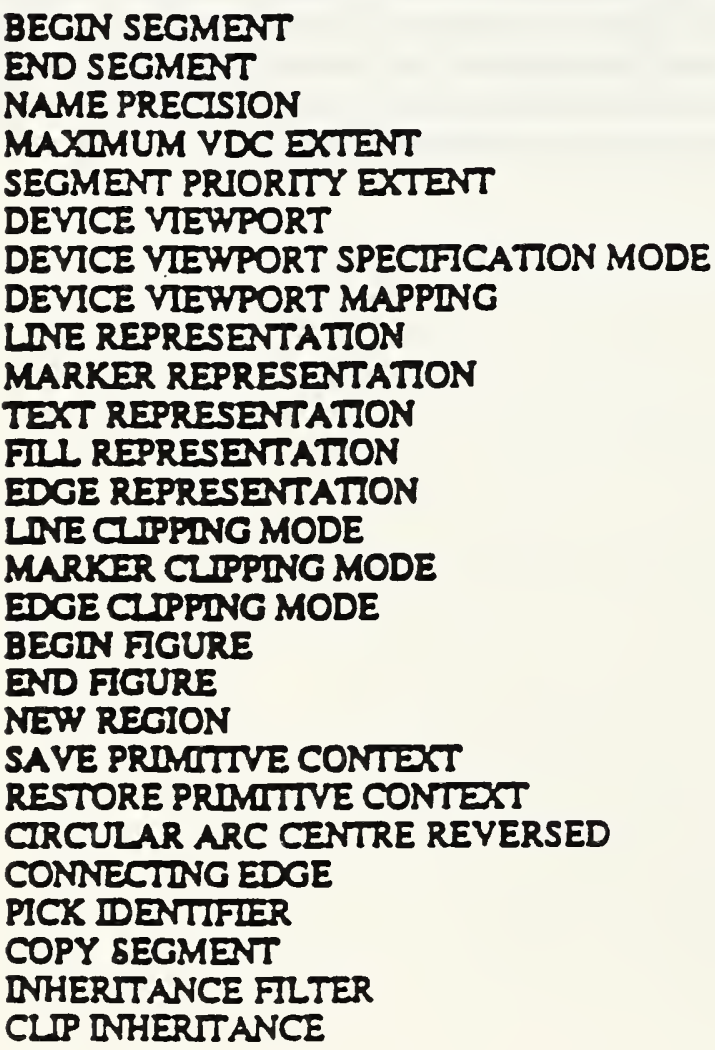 \\
\hline
\end{tabular}

BEGSEG
ENDSEG
NAMEPREC
MAXVDCEXT
SEGPRIEXT
DEVVP
DEVVPMODE
DEVVPMAP
LNEREP
MARKERREP
TEXTREP
FILREP
EDGEREP
LNECLPMODE
MARKERCLIPMODE
EDGECLIPMODE
BEGFIGURE
ENDFIGURE
NEWREGION
SAVEPRIMCONT
RESPRDMCONT
ARCCTRREY
CONNEDGE
PICKD
COPYSEG
DNHFLTER
CUPINH


SEGMENT TRANSFORMATION

SEGMENT VISIBRITY

SEGMENT HIGHLGHTING

SEGMENT DISPLAY PRIORTY

SEGMENT PICK PRIORTY
SEGTRANS

SEGVIS

SEGHIGHL

SEGDISPPRI

SEGPICKPRI

Page 15

Add the following to the end of sub-clause 6.2

BEGN SEGMENT

$$
\begin{aligned}
& \text { - BECSEG } \\
& \text { QSOFTSEP } \\
& \text { <TERM> } \\
& \text { QN:SEGID }
\end{aligned}
$$

END SECMENT

= ENDSEG <TERM>

Page 17

Add to the and of METAFILE EIEMENT LIST:

The words VER2STATIC. EXTENDED PRIMITIVES and VER2GKSMSTATIC may also be used in this string

Page 17

Add the following to the end of sub-clause 63

NAME PRECISION

$$
\begin{aligned}
& =\text { NAMEPREC } \\
& \text { SSOFTSEPS } \\
& \text { ULMMNINT } \\
& \text { ESEPS } \\
& \text { SI:MAXINTS } \\
& \text { STERM> }
\end{aligned}
$$

MAX VDC EXTENT

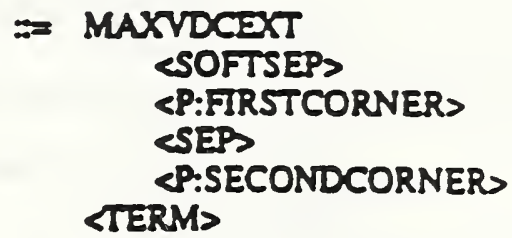

SEGMENT PRIORTY EXTENT $=$ SEGPRIEXT

ESOFTSEPS

CI:MINSEGPRL

¿SEP

<TERM>

UI:MAXSEGPRL

Page 18

Add the following wo the end of sub-clarse 6.4

DEVICE VIEPPORT

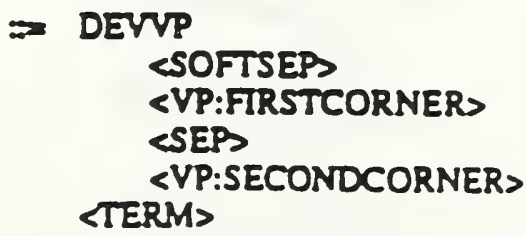

= DEYYP

QSOFTSEPS

<VP:FIRSTCORNER>

¿SEP

<YP:SECONDCORNER> <TERM>

DEVICE VIEWPORT SPECIFCATION

MODE

$::=$ DEVVP 


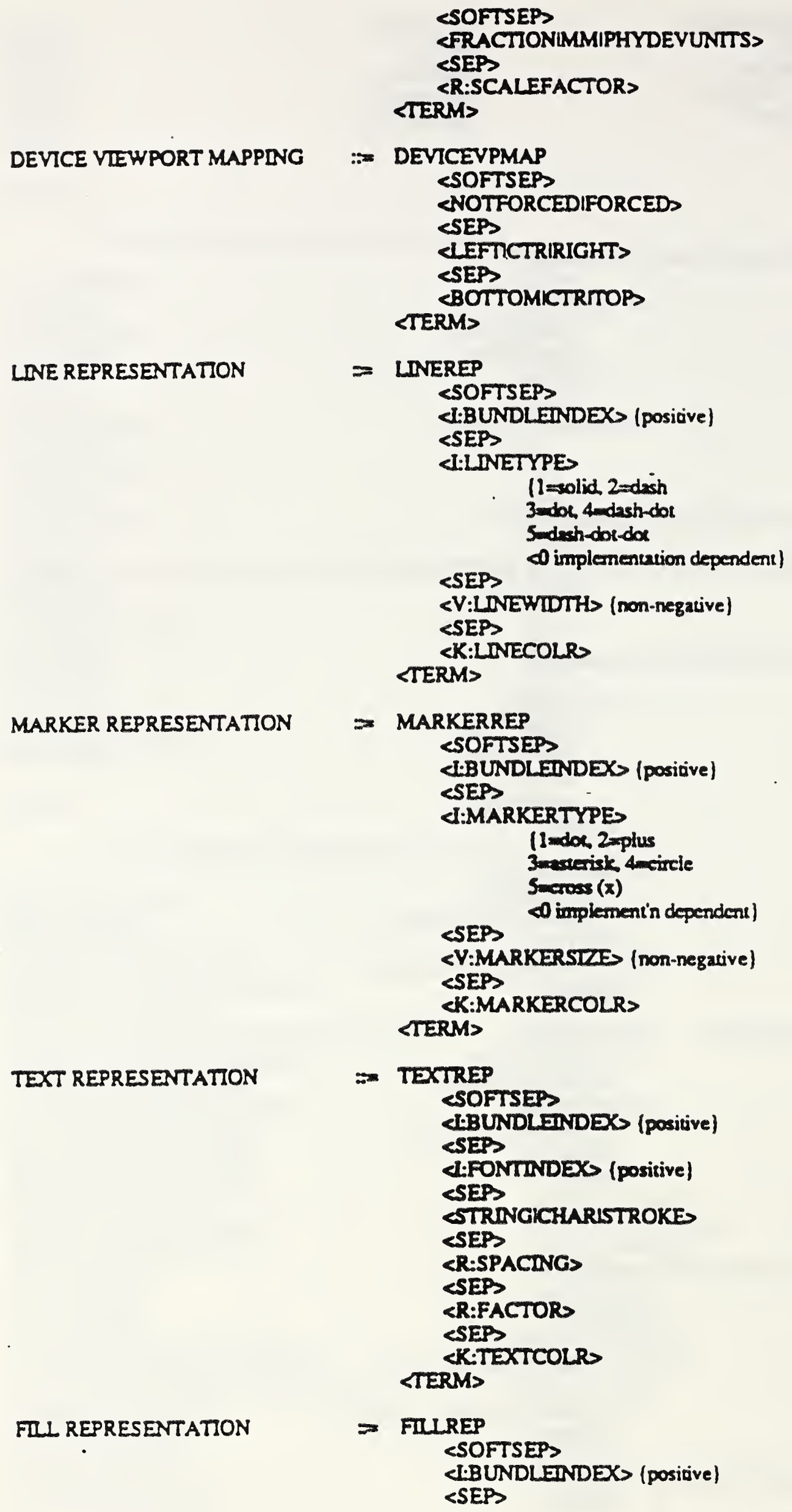




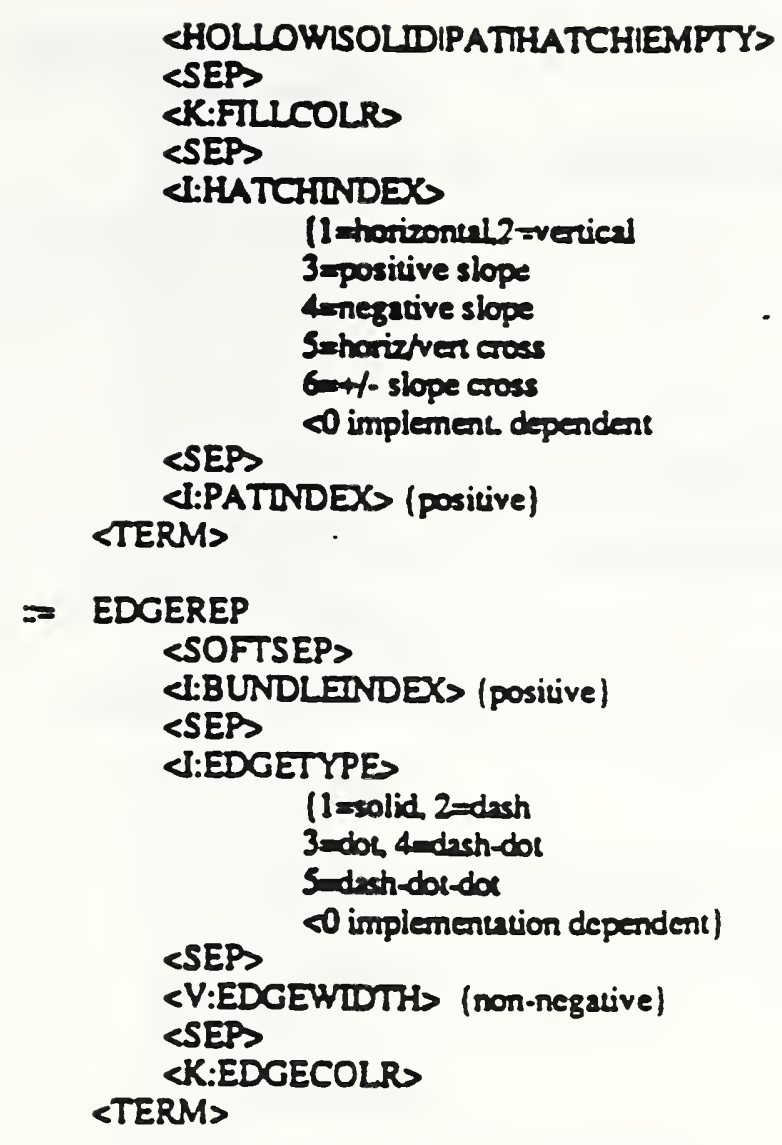

Page 19

Add the following to the end of sub-clause 65

LIVE CLIPPDNG MODE

MARKER CLIPPING MODE

EDGE CUIPPDNG MODE

BEGWN FIGURE

END FIGURE

NEW REGION

DMPLCTT EDGE VISIBILTY

SAVE PRIMITIVE CONTEXT
$=$ LDVECUPMODE SSOFTSEPS

¿LOCUSISHAPEILOCUSTHENSHAPE $>$ <TERM>

= MARKERCLPPMODE

ESOFTSEP>

¿LOCUSISHAPEILOCUSTHENSHAPE> <TERM>

- EDGECLIPMODE

ESOFTSEPS

LLCUSISHAPEROCUSTHENSHAPE> STERM>

$:=$ BEGFIGURE <TERM>

:= ENDFGURE <TERM>

$:=$ NEWREGION CTERM

$::=$. DMPLEDGEVIS SSOFTSEP> <TERM>

$::=$ SAVEPRDMCONT SSOFTSEP> 


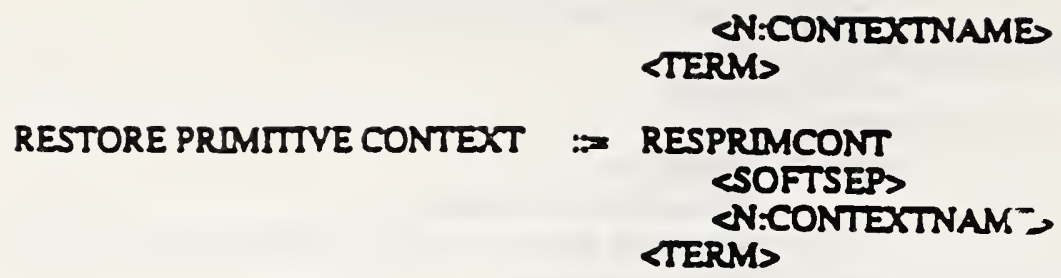

Add the following to the end of sub-clanse 6.6

CIRCULAR ARC CENTRE REV

$:=$ ARCCTRREV

$$
\text { STERM> }
$$

SCTRARCSPEC>

CONNECTING EDGE

$$
=\text { CONNEDG <TERM }>
$$

Page 28

Add the following to the end of sub-clause 6.7

PICX IDENTIFIER

$$
\begin{gathered}
=\text { PICKID } \\
\text { ESOFTSEP } \\
\text { AN:PICKID } \\
\text { CTERM> }
\end{gathered}
$$

Page 29

The following forms sub-clause 6.10

6.10 Encoding segment control and segment attribute elements COPY SEGMENT

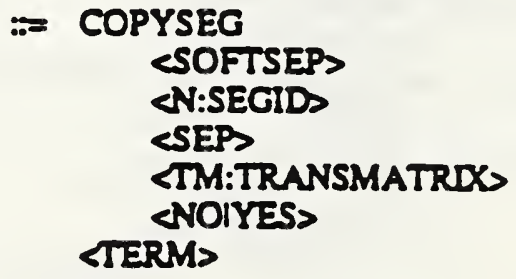


line type asf

line width asf

line colour asf

marke type asf

marter size asf

mixker colour asf

eexs fons index asf

text precision asf

chrrecter expension facrot asf

chereesers spacing asf

texs colour asf

interior style asf

in colour asf

hrich index asf

parem index asf

edge type af

dge width asf

edge colour asf

\section{CHARHEGHT}

CHARORII

TEXTPATHA

TEXTAUGN

FII.NDEX

DNTSTYE

FIULOLRI

HATCHINDEXI

PATINDEXI

EDGEINDEXI

EDGETYPE

EDGEWIDTH

EDGECOLRI

EDGEVISI

EDGECUPMODE

FILLREFERENCEPTI

PATSEAEI

AUXCOLRI

TRANSPARENCYI

LINEATTRI

MARKERATTRI

TEXTATTRI

CHARATTRI

FILLATTRI

EDGEATTRI

PATATTRI

OUTPUTCONTROU

PICKIDI

ALLATTRI *." and control

ALU

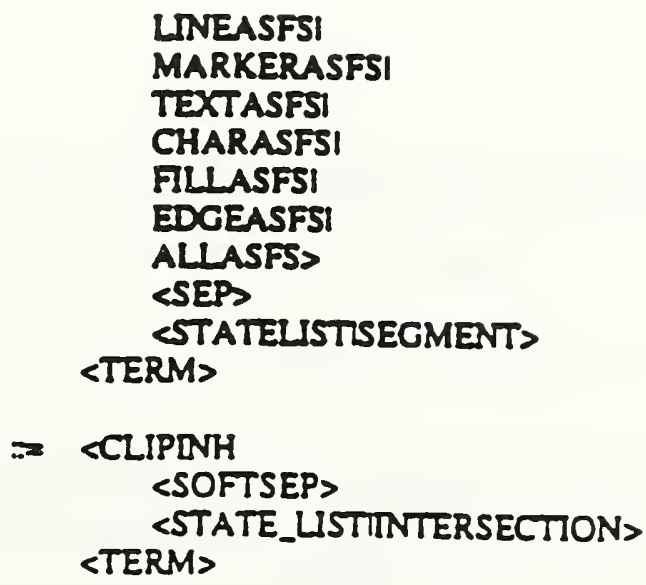




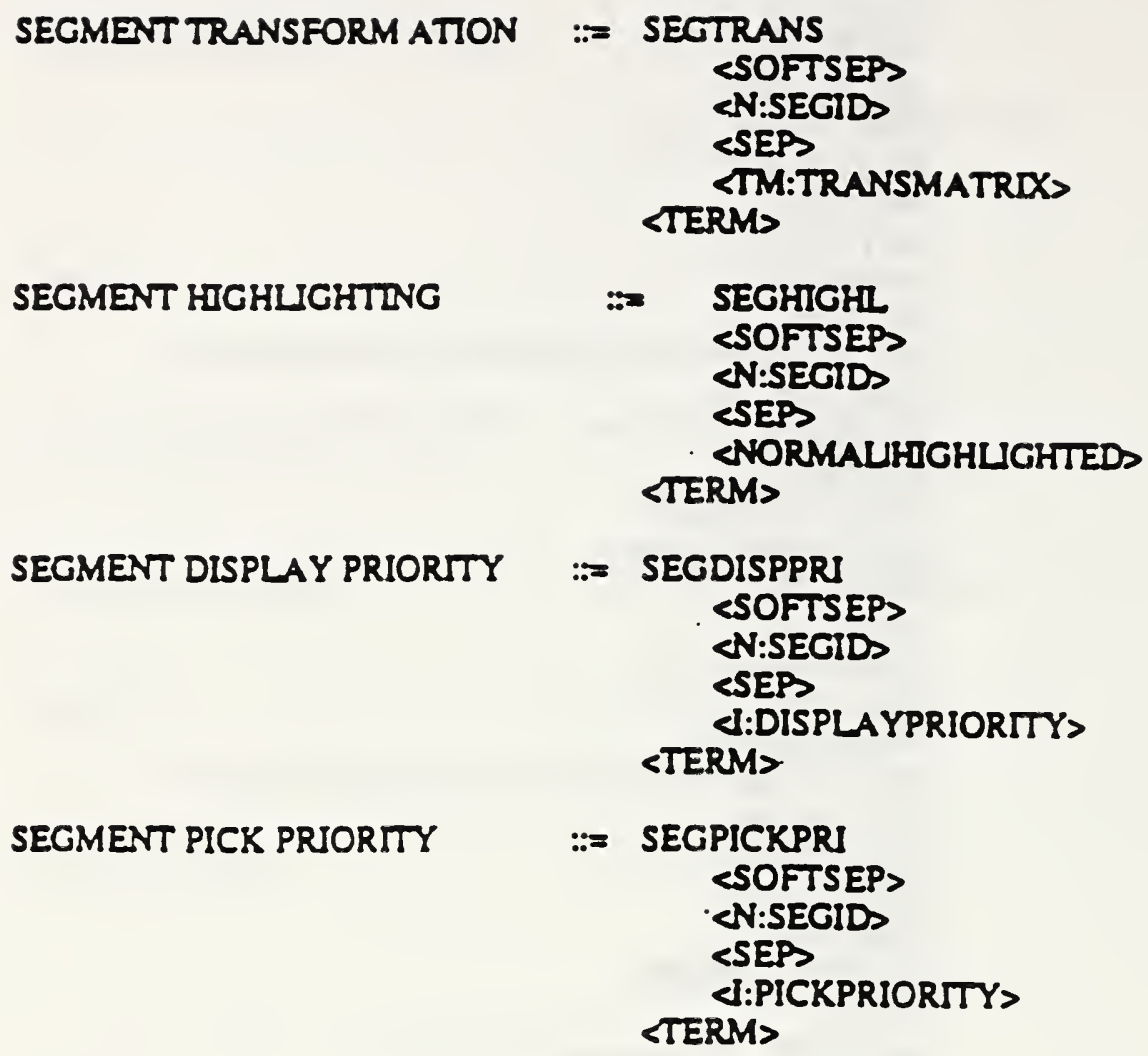

Page 30

Add the following wo the end of elause 7:

Piek identifier.

NAME PRECISION:

MININT 0

MAXINT 


\section{BIBLIOGRAPHIC DATA SHEET}

3. PUELCATION DATE

JULY 1990

4. TITLE AND SUCTITLE

Graphics Standards in the Computer-aided Acquisition and Logistic Support (CALS) Program,

Fiscal Year 1989, Volume 1: Test Requirements Doucment and Extended CGM (CGEM).

5. AUTHOR(S)

Daniel R. Benigni, Editor

6. PERFORMING ORGANIZATION (IF JOINT OR OTHEA THAN NIST, SEE INSTRUCTIONS)

U.S. DEPARTMENT OF COMMERCE

NATIONAL INSTITUTE OF STANDARDS AND TECHNOLOQY

GAITHERSBURG, MD 20890

7. CONTRACT/GRANT MUMBER

8. TYPE OF REPORT AND PEAIOD COVERED

NISTIR $10 / 88$ - $9 / 89$

9. SPONSORING ORGANIZATION MAME AND COMPLETE ADDRESS (STREET, CITY, STATE, ZIP)

Office of the Secretary of Defense

Production and Logistics/ Systems/ CALS

Room 3B322, Pentagon

Washington, D.C. 20301-8000

10. SUPPLEMENTARY NOTES

11. ABSTRACT (A 200-WOAD OR LESS FACTUAL SUMMARY OF MOST SIGMIFICANT INFORMATION. IF DOCUMENT INCLUDES A SIGMIFICANT BIBLOGRAPHY OR UTERATUAE SUAVEY, MENTION IT HERE,

The computer-aided Acquisition and Logistic Support (CALS) Program is a DoD Industry strategy to transition from paper-intensive acquisition and logistic processes to a highly automated and integrated mode of operation for the weapon systems of the 1990s. These volumes document the accomplishments of the Graphics Software Group of the National Institute of Standards and Technology (NIST) in support of computer graphics standards for CALS in FY89. They provide a progress report on continuing graphics standards efforts related to the Computer Graphics Metafile (CGM) standard. These reports are divided into two volumes: 1, Test Requirements Document and Extended CGM (CGEM); and 2, MIL-D-28003 Revisions and CGM Registration.

Volume 1. Completion of the first milestone toward the establishment of a comprehensive CGM (and MIL-D-28003) Test Method and ultimately a Testing Service, namely the creation of a Test Requirements Document, is detailed. This Test Requirements Document will provide the basis for developing conformance tests to determine compliance both with CGM and MIL-D-28003. Progress to inject CALS requirements into the Extended CGM (CGEM) is also detailed.

12. KEY WORDS (6 TO 12 ENTAIES; ALPAAETICAL ORDEA; CAPTALZE ONLY PROPER MAMES; AND SEPARATE KEY WORDS BY SEMICOLONS) application profile; CALS; CGEM; CGM; CGM registration; conformance testing; DoD; graphics; illutration data; MIL-D-28003; test requirements document

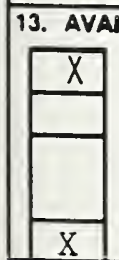

UNUMITED

FOR OFFICLAL DISTRIBUTION. DO NOT RELSASE TO MATIOMAL TECHMICAL INFORMATION SERVCE (NTIS).

ORDEA FROM SUPERINTENDENT OF DOCUMENTS, U.S. OOVERHMENT PAINTING OFFICE, WASHINOTON, DC 20402.

ORDEA FROM MATIONAL TECHMICAL IMFORMATION SERVCE (NTIS), SPRIMGFIEID, VA 22161.

\begin{tabular}{|l|} 
14. NUMBEA OF PAINTED PAGES \\
315 \\
\hline 15. PAICE \\
\end{tabular}




